\title{
Equações de Estimação Generalizadas com resposta binomial negativa: Modelando dados correlacionados de contagem com sobredispersão.
}

\author{
Clarissa Cardoso Oesselmann
}

\author{
DISSERTAÇÃO APRESENTADA \\ $\mathrm{AO}$
}

Instituto DE MATEMÁticA E EstatísticA

DA

Universidade De SÃo Paulo

PARA

OBTENÇÃO DO TÍTULO

DE

Mestre EM CIÊNCIAS

Programa: Mestrado em Estatística

Orientador: Gilberto A. Paula

Durante o desenvolvimento deste trabalho a autora recebeu auxílio financeiro da CNPq

São Paulo, outubro de 2016 


\section{Equações de Estimação Generalizadas com resposta binomial negativa: Modelando dados correlacionados de contagem com sobredispersão.}

\footnotetext{
Esta versão da dissertação/tese contém as correções e alterações sugeridas pela Comissão Julgadora durante a defesa da versão original do trabalho, realizada em 12/12/2016. Uma cópia da versão original está disponível no Instituto de Matemática e Estatística da Universidade de São Paulo.
}

Comissão Julgadora:

- Prof. Dr. Gilberto A. Paula - IME-USP

- Prof ${ }^{a}$. Dra ${ }^{a}$. Denise Aparecida Botter - IME-USP

- Prof ${ }^{a}$. Dra ${ }^{a}$. Maria Kelly Venezuela - INSPER 


\section{Agradecimentos}

Agradeço à minha mãe Nancy que me ouve quando preciso e que me dá puxão de orelha mesmo quando não quero. Sem seu incentivo não estaria aqui.

Agradeço ao meu irmão Daniel que me alimenta de comidas gostosas e de alegrias que me fizeram seguir em frente. E ao meu irmão Dante que me faz sentir criança novamente.

Agradeço à minha vó Osmary por ser a minha inspiração. À minha tia Le pelo cuidado que sempre me da.

Agradeço ao meu pai Gabriele e à minha madrasta Monique pelo acolhimento e pelos ensinamentos que me ajudaram nas transições da vida.

Agradeço à minha familia e amigos de Volta Redonda que sempre me fazem sentir em casa quando visito.

Agradeço aos meus amigos e colegas da UFF de Niterói que me fizeram chegar aqui e que me dão força sempre.

Agradeço aos amigos e colegas da USP que passaram por todas as fases desse mestrado comigo.

Agradeço aos novos amigos e colegas de Brasília que me ajudaram a criar um novo lar e a gostar do meu novo trabalho.

Agradeço ao professor Gilberto, meu orientador, pela paciência e ajuda sempre que precisei.

Agradeço aos professores da USP que me ensinaram e me educaram.

Agradeço ao Gui meu companheiro e meu amor, que pelas pequenas ações me ajuda e me faz feliz de uma forma que não imagina.

Agradeço a Deus pela força e paciência mesmo quando achava que não ia conseguir. 


\section{Resumo}

OESSELMANN, C. C. Equações de Estimação Generalizadas com resposta binomial negativa: Modelando dados correlacionados de contagem com sobredispersão. 2016. 125 f. Dissertação (Mestrado) - Instituto de Matemática e Estatística, Universidade de São Paulo, São Paulo, 2016.

Uma suposição muito comum na análise de modelos de regressão é a de respostas independentes. No entanto, quando trabalhamos com dados longitudinais ou agrupados essa suposição pode não fazer sentido. Para resolver esse problema existem diversas metodologias, e talvez a mais conhecida, no contexto não Gaussiano, é a metodologia de Equações de Estimação Generalizadas (EEGs), que possui similaridades com os Modelos Lineares Generalizados (MLGs). Essas similaridades envolvem a classificação do modelo em torno de distribuições da família exponencial e da especificação de uma função de variância. A única diferença é que nessa função também é inserida uma matriz trabalho que inclui a parametrização da estrutura de correlação dentro das unidades experimentais. O principal objetivo desta dissertação é estudar como esses modelos se comportam em uma situação específica, de dados de contagem com sobredispersão. Quando trabalhamos com MLGs esse problema é resolvido através do ajuste de um modelo com resposta binomial negativa (BN), e a ideia é a mesma para os modelos envolvendo EEGs. Essa dissertação visa rever as teorias existentes em EEGs no geral e para o caso específico quando a resposta marginal é BN, e além disso mostrar como essa metodologia se aplica na prática, com três exemplos diferentes de dados correlacionados com respostas de contagem.

Palavras-chave: Equações de Estimação Generalizadas, Binomial Negativa, Dados de Contagem, Sobredispersão. 


\section{Abstract}

OESSELMANN, C. C. Generalized Estimating Equations with negative binomial responses: Modeling correlated count data with overdispersion. 2016. 125 f. Dissertation (Master's degree) - Instituto de Matemática e Estatística, Universidade de São Paulo, São Paulo, 2010.

An assumption that is common in the analysis of regression models is that of independent responses. However, when working with longitudinal or grouped data this assumption may not have sense. To solve this problem there are several methods, but perhaps the best known, in the non Gaussian context, is the one based on Generalized Estimating Equations (GEE), which has similarities with Generalized Linear Models (GLM). Such similarities involve the classification of the model around the exponential family and the specification of a variance function. The only difference is that in this function is also inserted a working correlation matrix concerning the correlations within the experimental units. The main objective of this dissertation is to study how these models behave in a specific situation, which is the one on count data with overdispersion. When we work with GLM this kind of problem is solved by setting a model with a negative binomial response (NB), and the idea is the same for the GEE methodology. This dissertation aims to review in general the GEE methodology and for the specific case when the responses follow marginal negative binomial distributions. In addition, we show how this methodology is applied in practice, with three examples of correlated data with count responses.

Keywords: Generalized Estimating Equations, Negative Binomial, Count Data, Overdispersion. 


\section{Sumário}

$\begin{array}{ll}\text { Lista de Abreviaturas } & \text { ix }\end{array}$

Lista de Símbolos $\quad$ xi

Lista de Figuras $\quad$ xiii

Lista de Tabelas $\quad$ xv

1 Introdução $\quad 1$

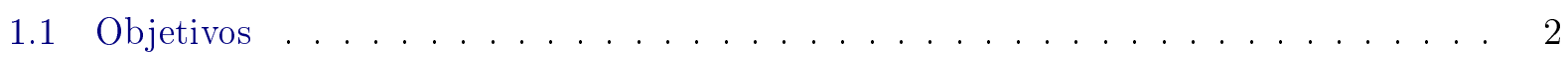

1.2 Bancos de Dados . . . . . . . . . . . . . . . . . . . . . . 2

1.2.1 Dados sobre Pacientes com Lepra . . . . . . . . . . . . . . . . 2

1.2.2 Dados sobre Pacientes Epilépticos . . . . . . . . . . . . . . . . 3

1.2.3 Dados sobre Pacientes do Germam Health Reform . . . . . . . . . . . . . . 4

1.3 Organização do Trabalho . . . . . . . . . . . . . . . . . . . 5

2 Conceitos de EEGs $\quad 7$

2.1 Funções de Estimação . . . . . . . . . . . . . . . . . . . . . . 7

2.2 Equações de Estimação . . . . . . . . . . . . . . . . . . . . . . 9

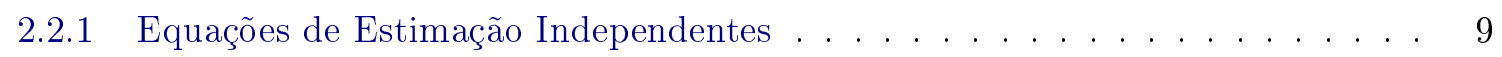

2.2 .2 Equações de Estimação Generalizadas . . . . . . . . . . . . . . . . . . 11

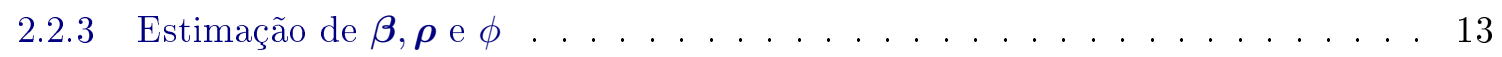

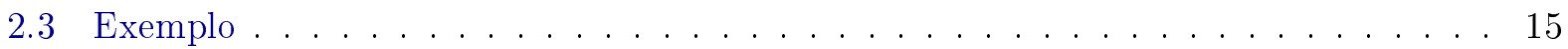

2.4 Métodos de Diagnóstico . . . . . . . . . . . . . . . 17

3 Extensão para a Binomial Negativa $\quad 21$

3.1 Modelos NB-C e NB2 . . . . . . . . . . . . . . . . . . . . . . 22

3.2 Ajuste de Modelos com Respostas Indenpendentes Binomial Negativa . . . . . . . . . 24

3.2 .1 Métodos de Diagnóstico . . . . . . . . . . . . . . . . . . . . . 26

3.3 Ajuste de Modelos com Respostas Correlacionadas Binomial Negativa . . . . . . . . 27

3.3.1 Métodos de Diagnóstico . . . . . . . . . . . . . . . . . . . . . . . 29

3.4 Aspectos Computacionais . . . . . . . . . . . . . . . . . . . . 29

3.5 Método Alternativo . . . . . . . . . . . . . . . . . . . . . 30

4 Aplicações $\quad 35$

4.1 Pacientes com Lepra . . . . . . . . . . . . . . . . . . . . 35 
4.1 .1 Análise Preliminar dos Dados . . . . . . . . . . . . . . . . 35

4.1 .2 Selecionando o Modelo . . . . . . . . . . . . . . . . . . . . . . . 39

4.1 .3 Interpretações . . . . . . . . . . . . . . . . . . . . . . . 45

4.1 .4 Método Alternativo . . . . . . . . . . . . . . . . . . 45

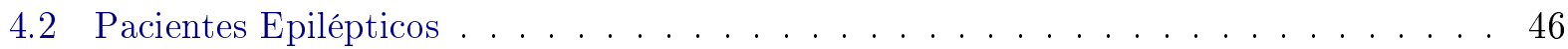

4.2 .1 Análise Preliminar dos Dados . . . . . . . . . . . . . . . . . 46

4.2 .2 Selecionando o Modelo . . . . . . . . . . . . . . . . . . . . . 51

4.2 .3 Melhorando o Modelo . . . . . . . . . . . . . . . . . . . . . . 57

4.2 .4 Método Alternativo . . . . . . . . . . . . . . . . . 59

4.3 Pacientes do German Health Reform . . . . . . . . . . . . . . . . . . 61

4.3.1 Análise Preliminar dos Dados . . . . . . . . . . . . . . . . . 61

4.3.2 Selecionando o Modelo . . . . . . . . . . . . . . . . . . . 69

4.3.3 Melhorando o Modelo . . . . . . . . . . . . . . . . . . . . 72

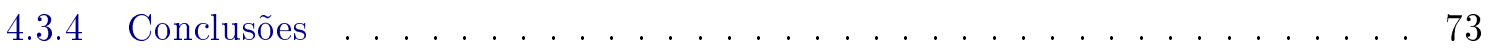

4.4 Método Alternativo . . . . . . . . . . . . . . . . . . . . 74

$\begin{array}{lll}5 & \text { Conclusões } & \mathbf{7 7}\end{array}$

5.1 Considerações Finais . . . . . . . . . . . . . . . . . . . 78

5.2 Sugestões para Pesquisas Futuras . . . . . . . . . . . . . . . . . 78

$\begin{array}{ll}\text { A Bancos de dados completos } & \mathbf{7 9}\end{array}$

A.1 Leprosy . . . . . . . . . . . . . . . . . . . . . . . 79

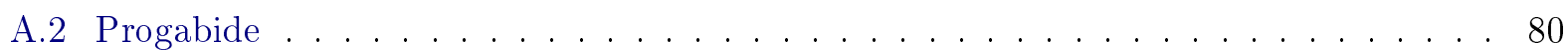

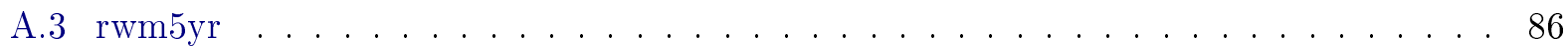

$\begin{array}{ll}\text { B Códigos do R e do SAS } & 87\end{array}$

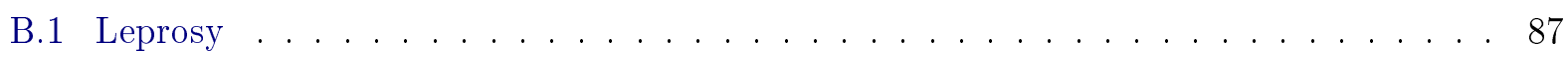

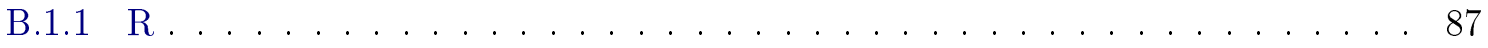

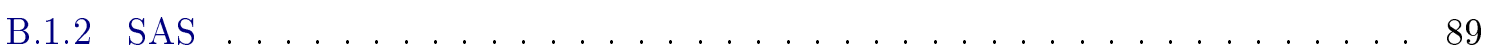

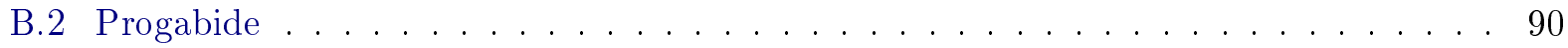

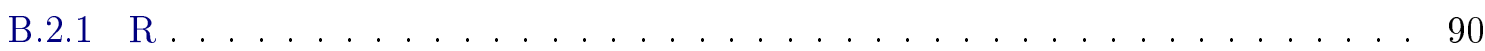

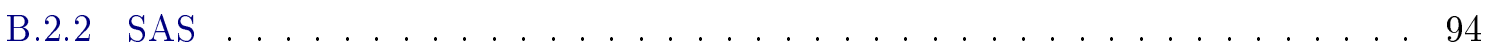

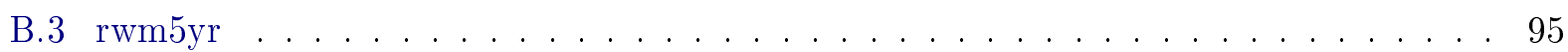

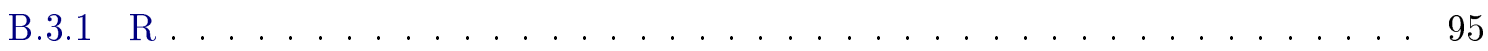

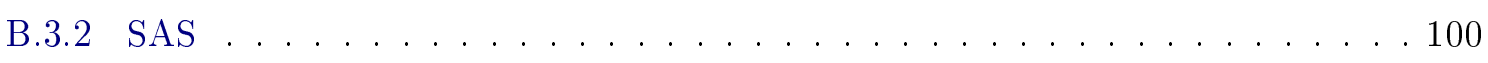

$\begin{array}{ll}\text { Referências Bibliográficas } & 103\end{array}$ 


\title{
Lista de Abreviaturas
}

\author{
EEG Equações de Estimação Generalizadas \\ MLG Modelos Lineares Generalizados \\ BN Binomial Negativa \\ FE Família Exponencial \\ EEI Equação de Estimação Independente \\ FDP Função Densidade de Probabilidade \\ NB2 Modelo Binomial Negativo tradicional \\ NB-C Modelo Binomial com função de ligação canônica
}




\title{
Lista de Símbolos
}

\author{
y $\quad$ Vetor de respostas \\ $\boldsymbol{\theta}$ Parâmetro de interesse \\ $\mathbb{R} \quad$ Conjunto dos números Reais \\ $\mathbb{N} \quad$ Conjunto dos números Naturais \\ $\Theta \quad$ Espaço Paramétrico \\ $m \quad$ Número de grupos ou de unidades experimentais \\ $n_{i} \quad$ Tamanho de cada grupo ou unidade experimental \\ $\psi \quad$ Função de estimação do vetor \\ $\Psi \quad$ Função de estimação da amostra \\ $p \quad$ Número de parâmetros \\ $\mathrm{E}(\cdot) \quad$ Esperança de uma variável \\ $\stackrel{\mathcal{P}}{\rightarrow} \quad$ Convergência em Probabilidade \\ $\stackrel{\mathcal{D}}{\rightarrow} \quad$ Convergência em Distribuição \\ lim Limite de uma função \\ J Matriz de informação de Godambe \\ S Matriz de Sensibilidade \\ V Matriz de Variabilidade \\ $\Psi^{*} \quad$ Função de estimação Ótima \\ Q Matriz não estocástica \\ $\mathbf{u} \quad$ Vetores com média zero e mutuamente independentes \\ X Valores das covaráveis \\ a Função de Variância \\ $\phi^{-1} \quad$ Parâmetro de dispersão \\ $\eta \quad$ Preditor Linear \\ $\boldsymbol{\beta} \quad$ Vetor de parâmetros desconhecidos \\ $\boldsymbol{\mu} \quad$ Vetor de médias \\ $\operatorname{Var}(\cdot) \quad$ Covariância de uma variável \\ $\operatorname{diag}(\cdot) \quad$ Matriz diagonal \\ W Matriz de pesos \\ A Matriz diagonal da função de variância \\ N Distribuição Normal \\ $\mathbf{R}(\boldsymbol{\rho}) \quad$ Matriz de correlação de trabalho \\ $\boldsymbol{\rho} \quad$ Correlação entre as observações dentro de um mesmo grupo \\ I Matriz Identidade \\ $\ell \quad$ Log-verossimilhança \\ $\mathcal{L} \quad$ Verossimilhança
}




\section{Lista de Figuras}

4.1 Boxplot robusto da contagem de bacilos. . . . . . . . . . . . . . . 35

4.2 Boxplot robusto da contagem de bacilos segundo o tratamento. . . . . . . . . . . 36

4.3 Boxplot robusto da contagem de bacilos segundo o momento. . . . . . . . . . . . 37

4.4 Boxplot robusto da contagem de bacilos segundo o tratamento e o momento. . . . . . 38

4.5 Gráficos de diagnóstico referentes ao ajuste no $\mathrm{R}$ do modelo log-linear de Poisson aos dados de pacientes com lepra. . . . . . . . . . . . . . . . . . . 40

4.6 Gráfico normal de probabilidades referente aos ajuste no $\mathrm{R}$ do modelo log-linear de Poisson aos dados de pacientes com lepra. . . . . . . . . . . . . . . . 40

4.7 Gráficos de diagnóstico referentes ao ajuste no $\mathrm{R}$ do modelo log-linear binomial negativo aos dados de pacientes com lepra. . . . . . . . . . . . . . . . 41

4.8 Gráfico normal de probabilidades referente ao ajuste no $\mathrm{R}$ do modelo log-linear binomial negativo aos dados de pacientes com lepra. . . . . . . . . . . . . . . 42

4.9 Métodos de diagnóstico referentes ao ajuste no R do modelo EEG-Poisson aos dados de pacientes com lepra. . . . . . . . . . . . . . . . . . 43

4.10 Boxplot robusto do número de ataques epilépticos. . . . . . . . . . . . . . . . . 46

4.11 Boxplot robusto do número de ataques epilépticos segundo o tratamento. . . . . . . . 47

4.12 Boxplot robusto do número de ataques epilépticos segundo o período. . . . . . . . . . 48

4.13 Boxplot robusto do número de ataques epilépticos segundo o tratamento e o período. 49

4.14 Gráfico de perfis do logaritmo do número de ataques epilépticos segundo o período. . 50

4.15 Gráfico da média do logaritmo do número de ataques epilépticos segundo o trata-

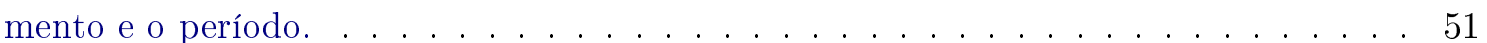

4.16 Gráficos do diagnóstico referentes ao ajuste no R do modelo log-linear de Poisson aos dados de pacientes com epilepsia. . . . . . . . . . . . . . . . . . 52

4.17 Gráfico normal de probabilidades referente ao ajuste no $\mathrm{R}$ do modelo log-linear de Poisson aos dados de pacientes com epilepsia. . . . . . . . . . . . . . . . . 53

4.18 Gráficos do diagnóstico referentes ao ajuste no $\mathrm{R}$ de um modelo log-linear BN aos dados de pacientes com epilepsia. . . . . . . . . . . . . . . . . . . 54

4.19 Gráfico normal de probabilidades referente ao ajuste no $\mathrm{R}$ de um modelo log-linear BN aos dados de pacientes com epilepsia. . . . . . . . . . . . . . . . . . 54

4.20 Gráficos de diagnóstico referentes ao ajuste do modelo EEG-Poisson no $\mathrm{R}$ aos dados de pacientes com epilepsia. . . . . . . . . . . . . . . . . 55

4.21 Gráfico normal de probabilidades referente ao ajuste do modelo EEG-Poisson no R aos dados de pacientes com epilepsia. . . . . . . . . . . . . . . . . . 56

4.22 Boxplot robusto do número de visitas ao médico. . . . . . . . . . . . . . . . 61 
4.23 Gráfico de dispersão entre o número de visitas ao médico e o número de visitas ao hospital. . . . . . . . . . . . . . . . . . . . . . . . 62

4.24 Boxplot robusto do número de visitas ao médico segundo o ano. . . . . . . . . . . . 63

4.25 Gráfico de dispersão entre o número de visitas ao médico e a idade do paciente. . 63

4.26 Boxplot robusto do número de visitas ao médico segundo a condição de trabalho. . . 64

4.27 Boxplot robusto do número de visitas ao médico segundo o gênero. . . . . . . . . . . 65

4.28 Boxplot robusto do número de visitas ao médico segundo o nível de educação. . . . . 66

4.29 Boxplot robusto do número de visitas ao médico segundo o estado civil. . . . . . . . 67

4.30 Boxplot robusto do número de visitas ao médico segundo a presença de filhos. . . . . 68 


\section{Lista de Tabelas}

1.1 Descrição das variáveis observadas no estudo clínico para analisar pacientes com lepra no sanatório Everly Childs, Filipinas. . . . . . . . . . . . . . . . 3

1.2 Descrição de parte dos dados do estudo clínico para analisar pacientes com epilepsia tratados com a droga antiepiléptica Progabide. . . . . . . . . . . . . . . . . . . 4

1.3 Resumo de parte dos dados do estudo longitudinal do German Health Reform para avaliar o número de visitas que pacientes fazem ao médico ao longo do ano. . . . . . 5

4.1 Medidas resumo da contagem de bacilos. . . . . . . . . . . . . . . . . . 35

4.2 Medidas resumo da contagem de bacilos segundo o tratamento. . . . . . . . . . . . 36

4.3 Medidas resumo da contagem de bacilos segundo o momento, antes ou depois do tratamento. . . . . . . . . . . . . . . . . . . . . 37

4.4 Medidas resumo da contagem de bacilos segundo tratamento e momento. . . . . . . . 38

4.5 Estimativas dos parâmetros do modelo linear normal ajustado no $\mathrm{R}$ aos dados de pacientes com lepra. . . . . . . . . . . . . . . . . . . . . . 39

4.6 Estimativas dos parâmetros do modelo log-linear de Poisson ajustado no $\mathrm{R}$ aos dados dos pacientes com lepra. . . . . . . . . . . . . . . . . . . . . . . . 39

4.7 Estimativas dos parâmetros do modelo log-linear binomial negativo ajustado no $\mathrm{R}$ aos dados de pacientes com lepra. . . . . . . . . . . . . . . . . . . . 41

4.8 Estimativas dos parâmetros referentes ao modelo EEG-Poisson ajustado no $\mathrm{R}$ aos dados de pacientes com lepra. . . . . . . . . . . . . . . . . . . . . . . 43

4.9 Estimativas dos parâmetros do modelo EEG-Poisson ajustado no SAS aos dados de pacientes com lepra. . . . . . . . . . . . . . . . . . . . . . . 44

4.10 Estimativa da matriz trabalho referente ao ajuste no SAS do modelo EEG-Poisson aos dados de pacientes com lepra. . . . . . . . . . . . . . . . . . . . 44

4.11 Estimativa dos parâmetros do modelo EEG-BN ajustado no SAS aos dados de pacientes com lepra. . . . . . . . . . . . . . . . . . . . . . . . . 44

4.12 Estimativa da matriz trabalho referente ao ajuste no SAS do modelo EEG-BN aos dados de pacientes com lepra. . . . . . . . . . . . . . . . . . . . . . 44

4.13 Estimativas dos parâmetros através do ajuste do modelo de cópulas Gaussianas com resposta Poisson ajustado no $\mathrm{R}$ aos dados de pacientes com lepra. . . . . . . . . . . 45

4.14 Estimativas dos prâmetros através do ajuste do modelo de cópulas Gaussianas com resposta binomial negativa ajustado no $\mathrm{R}$ aos dados de pacientes com lepra. . . . . 45

4.15 Medidas resumo da contagem de ataques epilépticos. . . . . . . . . . . . . . . . 46

4.16 Medidas resumo da contagem de ataques epilépticos segundo o tratamento. . . . . . 46 
4.17 Medidas resumo da contagem de ataques epilépticos por período. . . . . . . . . . . 47

4.18 Medidas resumo do número de ataques epilépticos segundo o período e o tratamento. 48

4.19 Estimativa de parâmetros do modelo linear normal ajustado no $\mathrm{R}$ aos dados de pacientes com epilepsia. . . . . . . . . . . . . . . . . . . 5 52

4.20 Estimativa dos parâmetros do modelo log-linear de Poisson ajustado no $\mathrm{R}$ aos dados de pacientes com epilepsia. . . . . . . . . . . . . . . . . . 52

4.21 Estimativas dos parâmetros referentes ao ajuste no $\mathrm{R}$ de um modelo log-linear BN aos dados de pacientes com epilepsia. . . . . . . . . . . . . . . . 5 53

4.22 Estimativas dos parâmetros do modelo EEG-Poisson ajustado no $\mathrm{R}$ aos dados de pacientes com epilepsia. . . . . . . . . . . . . . . . . . . 55

4.23 Matriz trabalho estimada referente ao modelo EEG-Poisson ajustado no $\mathrm{R}$ aos dados de pacientes com epilepsia. . . . . . . . . . . . . . . . . . 55

4.24 Estimativas dos parâmetros do modelo EEG-Poisson ajustado no SAS aos dados de pacientes com epilepsia. . . . . . . . . . . . . . . . 56

4.25 Matriz trabalho estimada referente ao modelo EEG-Poisson ajustado no SAS aos dados de pacientes com epilepsia. . . . . . . . . . . . . . . . . 56

4.26 Estimativa dos parâmetros do modelo EEG-BN ajustado no SAS aos dados de pacientes com epilepsia. . . . . . . . . . . . . . . . . . . . . 57 57

4.27 Matriz trabalho estimada referente ao modelo EEG-BN ajustado no SAS aos dados de pacientes com epilepsia. . . . . . . . . . . . . . . . . . . 57

4.28 Estimativas do modelo EEG-BN ajustado no SAS (com e sem o paciente \#207) aos dados de pacientes com epilepsia. . . . . . . . . . . . . . . . 58

4.29 Estimativas dos parâmetros do modelo EEG-BN ajustado no SAS aos dados de pacientes com epilepsia, sem o paciente $\# 207$. . . . . . . . . . . . . . . . 58

4.30 Matriz trabalho estimada referente ao modelo EEG-BN ajustado no SAS aos dados de pacientes com epilepsia, sem o paciente \#207. . . . . . . . . . . . . . . 58

4.31 Estimativas dos parâmetros do modelo EEG-BN ajustado no SAS aos dados de pacientes com epilepsia sem o paciente \#207 e com menor QIC. . . . . . . . . . . . . . 58

4.32 Matriz trabalho estimada referente ao modelo EEG-BN ajustado no SAS aos dados de pacientes com epilepsia, sem o paciente \#207 e com menor QIC. . . . . . . . . . . 59

4.33 Estimativas dos parâmetros do modelo de cópulas Gaussianas com resposta Poisson ajustado no $\mathrm{R}$ aos dados de pacientes com epilepsia.

4.34 Estimativas dos parâmetros do modelo de cópulas Gaussianas com resposta BN ajustado no $\mathrm{R}$ aos dados de pacientes com epilepsia. . . . . . . . . . . . . . . . . . 59

4.35 Estimativa dos parâmetros do modelo de cópulas Gaussianas com resposta BN ajustado no $\mathrm{R}$ aos dados de pacientes com epilepsia, sem o paciente $\# 207$. . . . . . . . . 59

4.36 Medidas resumo do número de visitas ao médico. . . . . . . . . . . . . . . . . . 61

4.37 Medidas resumo do número de visitas ao médico segundo o ano. . . . . . . . . . . . 62

4.38 Medidas resumo do número de visitas ao médico segundo a condição de trabalho. . . 64

4.39 Medidas resumo do número de visitas ao médico segundo o gênero. . . . . . . . . . . 65

4.40 Medidas resumo do número de visitas ao médico segundo o nível educaciional. . . . . 66

4.41 Medidas resumo do número de visitas ao médico segundo o estado civil. . . . . . . . . 67

4.42 Medidas resumo do número de visitas ao médico segundo a presença de filhos. . . . . 68 
4.43 Estimativas dos parâmetros do modelo linear normal ajustado no $\mathrm{R}$ aos dados de pacientes do German Health Reform. . . . . . . . . . . . . . . . . . . 69

4.44 Estimativas dos parâmetros do modelo log-linear de Poisson ajustado no $\mathrm{R}$ aos dados de pacientes do German Health Reform. . . . . . . . . . . . . . . . . . 70

4.45 Estimativas dos parâmetros do modelo log-linear binomial negativo ajustado no $\mathrm{R}$ aos dados de pacientes do German Health Reform. . . . . . . . . . . . . . . 70

4.46 Estimativas dos parâmetros do modelo EGG-Poisson ajustado no SAS aos dados de pacientes do German Health Reform. . . . . . . . . . . . . . . . . . 71

4.47 Matriz trabalho estimada referente ao ajuste no SAS do modelo EEG-Poisson aos dados de pacientes do German Health Reform. . . . . . . . . . . . . . . . . 71

4.48 Estimativas dos parâmetros do modelo EGG-BN ajustado no SAS aos dados de pacientes do German Health Reform. . . . . . . . . . . . . . . . . 71

4.49 Matriz trabalho estimada referente ao ajuste no SAS do modelo EEG-BN aos dados de pacientes do German Health Reform. . . . . . . . . . . . . . . . . . 72

4.50 Estimativas dos parâmetros do modelo EGG-BN ajustado no SAS aos dados de pacientes do German Health Reform, com matriz de correlação Independente. . . . . 72

4.51 Estimativas dos parâmetros do modelo EGG-BN ajustado no SAS aos dados de pacientes do German Health Reform, com matriz de correlação Independente e sem a variável Casado. . . . . . . . . . . . . . . . . . . . 73

4.52 Estimativas dos parâmetros do modelo de cópulas Gaussianas com resposta Poisson ajustado no R aos dados de pacientes do German Health Reform. . . . . . . . . . . . 74

4.53 Estimativas dos parâmetros do modelo de cópulas Gaussianas com resposta BN ajustado no $\mathrm{R}$ aos dados de pacientes do German Health Reform. . . . . . . . . . . . . 75

A.1 Leprosy: Banco de dados . . . . . . . . . . . . . . . . . . . . . . . . . 79

A.2 Progabide: Banco de dados . . . . . . . . . . . . . . . . 80 


\section{Capítulo 1}

\section{Introdução}

Quando se está trabalhando com modelos baseados em verossimilhança é comum fazer a suposição básica de que as respostas são independentes. Essa é uma suposição razoável para talvez a maioria dos estudos. Contudo, para alguns tipos de estudo essa suposição não é viável, por exemplo para estudos longitudinais ou estudos com dados agrupados.

Para ilustrar, supor que estamos trabalhando com dados agrupados (estrutura de painel), analisando o número de domicílios com banda larga numa cidade. Os domicílios podem ser agrupados por bairro, e é razoável esperar que os domicílios que estão em um mesmo bairro tenham uma correlação entre si, não podendo mais ser considerado que essas observações sejam independentes.

Da mesma forma, se temos dados longitudinais, por exemplo coletados ao longo do tempo para cada indivíduo, as observações relacionadas a um mesmo indivíduo nos diferentes períodos de tempo, muito provavelmente estão mais correlacionadas entre si do que se forem resultantes de um mesmo tratamento aplicado a indivíduos diferentes. Logo, é muito razoável que essa suposição seja levada em conta nas análises estatísticas.

Quando se fala sobre ajustar um modelo que lide com eventos longitudinais ou agrupados, a metodologia conhecida como Equações de Estimação Generalizadas (EEGs) é talvez a mais popular no contexto não Gaussiano.

As EEGs referem-se a um método de estimação proposto por Liang e Zeger (1986). Diferentemente do modelo de efeitos aleatórios, que é específico para indivíduos, as EEGs focam na estimação de coeficientes que tenham uma interpretação populacional (Weiss, 2005). A parte essencial desse método é a especificação de uma estrutura de correlação denominada matriz trabalho, que irá estimar a correlação extra mencionada no modelo.

A motivação para definir as EEGs é propor uma "função de variância" $\mathrm{V}(\boldsymbol{\mu})$ dos modelos lineares generalizados, de forma que a matriz trabalho, denotada por $\mathrm{R}$, contendo a estrutura de correlação em cada grupo seja incorporada ao modelo. Essa função de variância (em forma matricial) é definida por:

$$
\mathrm{V}^{*}(\boldsymbol{\mu})=\mathrm{V}(\boldsymbol{\mu})^{\frac{1}{2}} \mathbf{R V}(\boldsymbol{\mu})^{\frac{1}{2}},
$$

em que $\mathrm{V}(\boldsymbol{\mu})$ é a função de variância (em forma matricial) correspondente à distribuição das respostas se essas forem independentes (Hilbe, 2011). Uma vantagem em utilizar a abordagem de EEGs é que a matriz trabalho $\mathrm{R}$ pode ser arbitrariamente parametrizada, ou mesmo, não ser estruturada. Em geral, dados agrupados e analisados através de EEGs são modelados de forma similar aos MLGs, ou seja, a variável resposta pode ter qualquer distribuição marginal pertencente à família exponencial, ou mesmo, ter apenas os dois primeiros momentos conhecidos. Para essa dissertação em particular, vamos focar em dados com variável resposta marginal de contagem, ou seja, com resposta Poisson e, quando apresentar sobredispersão, binomial negativa. 


\subsection{Objetivos}

O objetivo principal deste trabalho é dissertar sobre as EEGs e de forma particular sua aplicação para dados de contagem longitudinais e com sobredispersão, ou seja, quando a variável resposta é binomial negativa. Além disso, também iremos fazer a aplicação desse método para diferentes dados de contagem.

\subsection{Bancos de Dados}

Abaixo descrevemos os bancos de dados que serão analisados no trabalho:

- Exemplo 1: apenas 2 momentos, mas sem controle de tempo.

- Exemplo 2: 4 momentos equidistantes de tempo balanceado.

- Exemplo 3: 5 momentos de tempo desbalanceado.

\subsubsection{Dados sobre Pacientes com Lepra}

Os dados a seguir são oriundos de um estudo clínico que incluía 30 pacientes com lepra no Sanatório Eversly Childs nas Filipinas (Cui e Feng, 2008; Snedecor e Cochran, 1967).Participantes no estudo foram aleatorizados para receber um tratamento com antibióticos ou placebo. Antes da escolha, foram coletados dados base sobre o número de bacilos leprosos no corpo do paciente. Depois de vários meses de tratamento o número de bacilos leprosos foi medido de novo. A Tabela 1.1 resume os dados dos pacientes do estudo. As variáveis do banco são as seguintes:

- id: número de identificação do paciente;

- count: contagem de bacilos leprosos no paciente;

- time: indicador de que os dados foram coletados antes (0) ou depois (1) da escolha aleatória do tratamento; e

- drug: indicador de que o paciente recebeu o tratamento (1) ou o placebo (0).

Esses dados podem ser considerados longitudinais, pois temos uma contagem em um mesmo paciente em dois períodos no tempo. Por isso, teríamos como variável resposta a contagem de bacilos leprosos (count) e os grupos seriam cada paciente (id) e queremos verificar se o tempo (time) ou o tratamento (drug) influenciam na contagem média de bacilos leprosos. 
Tabela 1.1: Descrição das variáveis observadas no estudo clínico para analisar pacientes com lepra no sanatório Everly Childs, Filipinas.

\begin{tabular}{|c|c|c|c|c|c|c|c|}
\hline id & count & time & drug & $\overline{\text { id }}$ & count & time & drug \\
\hline 1 & 11 & 0 & 0 & 16 & 6 & 0 & 0 \\
\hline 1 & 6 & 1 & 0 & 16 & 4 & 1 & 0 \\
\hline 2 & 6 & 0 & 1 & 17 & 8 & 0 & 1 \\
\hline 2 & 0 & 1 & 1 & 17 & 4 & 1 & 1 \\
\hline 3 & 16 & 0 & 1 & 18 & 16 & 0 & 1 \\
\hline 3 & 13 & 1 & 1 & 18 & 12 & 1 & 1 \\
\hline 4 & 8 & 0 & 0 & 19 & 10 & 0 & 0 \\
\hline 4 & 0 & 1 & 0 & 19 & 13 & 1 & 0 \\
\hline 5 & 6 & 0 & 1 & 20 & 19 & 0 & 1 \\
\hline 5 & 2 & 1 & 1 & 20 & 14 & 1 & 1 \\
\hline 6 & 13 & 0 & 1 & 21 & 12 & 0 & 1 \\
\hline 6 & 10 & 1 & 1 & 21 & 5 & 1 & 1 \\
\hline 7 & 5 & 0 & 0 & 22 & 6 & 0 & 0 \\
\hline 7 & 2 & 1 & 0 & 22 & 1 & 1 & 0 \\
\hline 8 & 7 & 0 & 1 & 23 & 8 & 0 & 1 \\
\hline 8 & 3 & 1 & 1 & 23 & 9 & 1 & 1 \\
\hline 9 & 11 & 0 & 1 & 24 & 12 & 0 & 1 \\
\hline 9 & 18 & 1 & 1 & 24 & 16 & 1 & 1 \\
\hline 10 & 14 & 0 & 0 & 25 & 11 & 0 & 0 \\
\hline 10 & 8 & 1 & 0 & 25 & 8 & 1 & 0 \\
\hline 11 & 8 & 0 & 1 & 26 & 5 & 0 & 1 \\
\hline 11 & 1 & 1 & 1 & 26 & 1 & 1 & 1 \\
\hline 12 & 9 & 0 & 1 & 27 & 7 & 0 & 1 \\
\hline 12 & 5 & 1 & 1 & 27 & 1 & 1 & 1 \\
\hline 13 & 19 & 0 & 0 & 28 & 3 & 0 & 0 \\
\hline 13 & 11 & 1 & 0 & 28 & 0 & 1 & 0 \\
\hline 14 & 18 & 0 & 1 & 29 & 15 & 0 & 1 \\
\hline 14 & 18 & 1 & 1 & 29 & 9 & 1 & 1 \\
\hline 15 & 21 & 0 & 1 & 30 & 12 & 0 & 1 \\
\hline 15 & 23 & 1 & 1 & 30 & 20 & 1 & 1 \\
\hline
\end{tabular}

\subsubsection{Dados sobre Pacientes Epilépticos}

Os dados são de 59 pacientes que sofriam com epilepsia e foram inscritos em um estudo clínico aleatório para testar uma droga antiepilética, Progabide.

Participantes do estudo foram escolhidos aleatoriamente para receber o tratamento com a droga Progabide ou para receber o placebo, como um adicional para a quimioterapia antiepilética padrão. Antes de receberem o tratamento, dados base com o número de ataques epilépticos que ocorreram no intervalo de 8 semanas anteriores foram registrados. Contagem de episódios epilépticos durante um intervalo de 2 semanas que antecederam cada uma das 4 visitas clínicas pós-aleatorização foram também registradas.

Um resumo de como os dados estão armazenados no banco de dados está descrito na Tabela 1.2 , e as variáveis são as seguintes:

- id: número identificador do paciente;

- trat: indicador de que o paciente recebeu o tratamento com progabide (1) ou com o placebo $(0)$;

- seizures: número de ataques epilépticos registrados no tempo t; e 
- t: indicador sobre qual momento do tempo foi registrada cada informação (0: dados base nas primeiras 8 semanas; 1: primeiras 2 semanas pós-aleatorização; 2: 2 semanas seguintes; 3: 2 semanas seguintes; e 4: 2 semanas seguintes).

Esses dados também são considerados longitudinais, mas desta vez os pacientes tiveram um acompanhamento maior durante o estudo, com vários registros durante o período do estudo. Dessa forma fica mais fácil verificar a correlação entre os registros para cada paciente. A variável resposta é o número de ataques epilépticos em cada tempo (seizures), a variável id é o identificador dos grupos e o que queremos analisar é se o tratamento produziu efeito (trat), no sentido de reduzir o número médio de ataques epilépticos.

Tabela 1.2: Descrição de parte dos dados do estudo clínico para analisar pacientes com epilepsia tratados com a droga antiepiléptica Progabide.

\begin{tabular}{cccc|cccc}
\hline id & trat & seizures & $\mathrm{t}$ & id & trat & seizures & $\mathrm{t}$ \\
\hline 1 & 0 & 11 & 0 & 29 & 1 & 76 & 0 \\
1 & 0 & 5 & 1 & 29 & 1 & 11 & 1 \\
1 & 0 & 3 & 2 & 29 & 1 & 14 & 2 \\
1 & 0 & 3 & 3 & 29 & 1 & 9 & 3 \\
1 & 0 & 3 & 4 & 29 & 1 & 8 & 4 \\
2 & 0 & 11 & 0 & 30 & 1 & 38 & 0 \\
2 & 0 & 3 & 1 & 30 & 1 & 8 & 1 \\
2 & 0 & 5 & 2 & 30 & 1 & 7 & 2 \\
2 & 0 & 3 & 3 & 30 & 1 & 9 & 3 \\
2 & 0 & 3 & 4 & 30 & 1 & 4 & 4 \\
3 & 0 & 6 & 0 & 31 & 1 & 19 & 0 \\
3 & 0 & 2 & 1 & 31 & 1 & 0 & 1 \\
3 & 0 & 4 & 2 & 31 & 1 & 4 & 2 \\
3 & 0 & 0 & 3 & 31 & 1 & 3 & 3 \\
3 & 0 & 5 & 4 & 31 & 1 & 0 & 4 \\
$\vdots$ & $\vdots$ & $\vdots$ & $\vdots$ & $\vdots$ & $\vdots$ & $\vdots$ & $\vdots$ \\
\hline
\end{tabular}

\subsubsection{Dados sobre Pacientes do Germam Health Reform}

Consiste em 19609 observações dos dados do German Health Reform que cobrem um período de 5 anos de 1984 até 1988. Nem todos os pacientes do estudo participaram de todos os 5 anos (Hilbe, 2014), gerando um planejamento desbalanceado para matriz trabalho.

A forma como os dados dos 7 primeiros pacientes está armazenada no banco de dados é apresentada na Tabela 1.3, e as variáveis são as seguintes:

- id: identificador do paciente;

- docvis: número de visitas feitas por um paciente a um médico durante o ano;

- hospvis: número de visitas feitas por um paciente a um hospital durante o ano;

- year: ano da observação que varia de 1984 a 1988;

- age: idade do paciente que varia de 25 até 64 anos;

- outwork: indicador se o paciente estava trabalhando (1) ou não estava trabalhando(0);

- female: indicador do gênero do paciente, feminino (1) ou masculino (0);

- married: indicador se o paciente é casado (1) ou não casado (0); 
- kids: indicador se o paciente tem filhos (1) ou não tem filhos (0);

- edlevel: variável categórica de 1 a 4 que foi separada em 4 variáveis binárias de edlevel1 até edlevel4, sendo que o edlevel1 representa pacientes que não terminaram o ensino médio, edlevel2 pacientes que terminaram o ensino médio, edlevel3 pacientes que terminaram o ensino superior e edlevel4 pacientes que possuem pós-graduação.

Esses dados também são longitudinais, pois são acompanhados por vários anos. Um diferencial em relação aos dados anteriores é que esses dados não são balanceados, ou seja, cada paciente tem um número diferente de observações além do número alto de observações. A variável resposta nesse caso é o número de visitas ao médico durante o ano (docvis), a variável id é o identificador dos pacientes e o que queremos analisar é quais variáveis dependentes explicam a média da variável resposta.

Tabela 1.3: Resumo de parte dos dados do estudo longitudinal do German Health Reform para avaliar o número de visitas que pacientes fazem ao médico ao longo do ano.

\begin{tabular}{ccccccccccccc}
\hline id & docvis & hospvis & year & age & outwork & female & married & kids & edlevel1 & edlevel2 & edlevel3 & edlevel4 \\
\hline 1 & 1 & 0 & 1984 & 54 & 0 & 0 & 1 & 0 & 0 & 0 & 1 & 0 \\
1 & 0 & 0 & 1985 & 55 & 0 & 0 & 1 & 0 & 0 & 0 & 1 & 0 \\
1 & 0 & 0 & 1986 & 56 & 0 & 0 & 1 & 0 & 0 & 0 & 1 & 0 \\
2 & 0 & 0 & 1984 & 44 & 1 & 1 & 1 & 0 & 1 & 0 & 0 & 0 \\
2 & 1 & 0 & 1985 & 45 & 1 & 1 & 1 & 0 & 1 & 0 & 0 & 0 \\
2 & 2 & 0 & 1986 & 46 & 1 & 1 & 1 & 0 & 1 & 0 & 0 & 0 \\
2 & 1 & 0 & 1988 & 48 & 1 & 1 & 1 & 0 & 1 & 0 & 0 & 0 \\
3 & 0 & 0 & 1984 & 58 & 1 & 1 & 0 & 0 & 1 & 0 & 0 & 0 \\
3 & 0 & 0 & 1986 & 60 & 1 & 1 & 0 & 0 & 1 & 0 & 0 & 0 \\
3 & 10 & 0 & 1987 & 61 & 1 & 1 & 0 & 0 & 1 & 0 & 0 & 0 \\
3 & 3 & 0 & 1988 & 62 & 1 & 1 & 0 & 0 & 1 & 0 & 0 & 0 \\
4 & 4 & 0 & 1985 & 29 & 0 & 1 & 0 & 0 & 0 & 0 & 0 & 1 \\
5 & 1 & 0 & 1987 & 27 & 1 & 0 & 0 & 0 & 1 & 0 & 0 & 0 \\
5 & 2 & 0 & 1988 & 28 & 1 & 0 & 0 & 0 & 1 & 0 & 0 & 0 \\
6 & 2 & 0 & 1985 & 25 & 0 & 0 & 0 & 1 & 1 & 0 & 0 & 0 \\
6 & 3 & 1 & 1986 & 26 & 0 & 0 & 1 & 1 & 1 & 0 & 0 & 0 \\
6 & 0 & 0 & 1987 & 27 & 0 & 0 & 1 & 1 & 1 & 0 & 0 & 0 \\
6 & 1 & 0 & 1988 & 28 & 0 & 0 & 1 & 1 & 1 & 0 & 0 & 0 \\
7 & 0 & 0 & 1987 & 26 & 0 & 1 & 1 & 1 & 1 & 0 & 0 & 0 \\
7 & 0 & 0 & 1988 & 27 & 1 & 1 & 1 & 1 & 1 & 0 & 0 & 0 \\
\hline
\end{tabular}

\subsection{Organização do Trabalho}

No Capítulo 2 revisamos alguns conceitos sobre Equações de Estimação Generalizadas, com o objetivo de entender como essa metodologia pode ser aplicada às distribuições da família exponencial.

No Capítulo 3 discutimos como os conceitos de EEGs podem ser aplicados para a distribuição binomial negativa.

No Capítulo 4 colocamos em prática os conceitos vistos anteriormente para os bancos de dados descritos neste capítulo.

Finalmente, no Capítulo 5 discutimos algumas conclusões obtidas neste trabalho. Analisamos as vantagens e desvantagens do método proposto bem como possíveis trabalhos futuros. 


\section{Capítulo 2}

\section{Conceitos de EEGs}

Equações de Estimação Generalizadas se referem a um método de estimação que foi primeiramente proposto por Liang e Zeger (1986). Essa metodologia pode ser interpretada como uma extensão para dados de painel dos modelos lineares generalizados, apesar de não ser necessária a especificação de que a variável resposta seja da FE (família exponencial), basta caracterizar a média e a variância como em um MLG. Na raiz da especificação do modelo, a função de variância (idêntica à especificação do MLG) é fatorada para incluir a parametrização da estrutura de correlação de dentro do painel.

A proposta inicial de Liang e Zeger (1986) foi introduzir o método EEGs para a estimação somente da média, mas posteriormente Lipsitz et al. (1991) descreveram um método no qual a estimação da média e da correlação ocorrem simultaneamente, chamado de EEGs2. Outro autor (Zorn, 2001) adverte que esse método somente deve ser usado quando a estrutura de correlação da matriz trabalho for conhecida.

Outra proposta para o método EEGs, é utilizá-lo para modelar o parâmetro de dispersão, também chamado de EEGs3. Artes (1997) desenvolveu equações de estimação para as três propostas, EEGs1, EEGs2 e EEGs3, para modelos de dispersão com medidas repetidas. Song e Tan (2000) apresentaram equações de estimação na proposta EEGs2, para a distribuição simplex de Barndorff-Nielsen e Jørgensen (1991), e logo em seguida Song et al. (2004) mostraram para essa mesma distribuição, sob a proposta de EEGs3, como a modelagem do parâmetro de dispersão altera as estimativas dos parâmetros de regressão.

Para este trabalho será usada a abordagem proposta por Liang e Zeger (1986), que é conhecida como EEGs1, mas que iremos chamar somente de EEGs. No entanto, antes de falarmos de equações de estimação, precisamos falar de funções de estimação.

Tecnicamente definimos uma função de estimação como uma função mensurável dos dados $(\mathbf{y})$ e dos parâmetros de interesse $(\boldsymbol{\theta})$. Essas funções são construídas de forma que suas raízes sejam estimadores dos parâmetros. Uma equação de estimação é obtida quando igualamos tais funções de estimação a zero. O maior problema é garantir que os estimadores encontrados através dessas equações possuam boas propriedades, ou seja, que sejam consistentes e com distribuição assintótica conhecida.

Faremos uma revisão das funções de estimação e veremos resultados encontrados em Artes (1997) e Jørgensen e Labouriau . Esses resultados estão apresentados de forma mais completa na dissertação de mestrado de Venezuela (2003) (vide também Venezuela et al. , 2007).

\subsection{Funções de Estimação}

Considerando $(\mathcal{X}, \mathcal{A}, \mathcal{P})$ um espaço de probabilidade, com $\mathcal{X} \in \mathbb{R}$ e $\mathcal{P}=\left\{P_{\theta}: \boldsymbol{\theta} \in \Theta \subseteq \mathbb{R}^{p}\right\}$, para algum $p \in \mathbb{N}$ ( $p$ é um valor fixado referente à dimensão do espaço paramétrico $\Theta$ ), uma função $\boldsymbol{\psi}: \mathcal{X} \times \Theta \longrightarrow \mathbb{R}^{p}$, é uma função de estimação se, para cada $\boldsymbol{\theta} \in \Theta, \boldsymbol{\psi}(\cdot ; \boldsymbol{\theta})=\left(\psi_{1}, \ldots, \psi_{p}\right)^{\top}$ é uma variável aleatória.

Assumindo uma amostra de $m$ vetores aleatórios independentes $\mathbf{y}_{i}=\left(y_{i 1}, \ldots, y_{i n_{i}}\right)^{\top}, i=$ 
$1, \ldots, m$, e que a cada vetor esteja associada uma função de estimação $\boldsymbol{\psi}_{i}$, estendemos o conceito de função de estimação para a amostra da seguinte forma:

$$
\boldsymbol{\Psi}_{m}(\mathbf{y} ; \boldsymbol{\theta})=\sum_{i=1}^{m} \boldsymbol{\psi}_{i}\left(\mathbf{y}_{i} ; \boldsymbol{\theta}\right)
$$

com dimensão $p \times 1$, sendo $\mathbf{y}=\left(\mathbf{y}_{1}^{\top}, \ldots, \mathbf{y}_{m}^{\top}\right)^{\top}$ um vetor $N \times 1, N=\sum_{i=1}^{m} n_{i}$.

A seguir apresentamos algumas propriedades de funções de estimação denotando $\boldsymbol{\Psi}_{m}(\mathbf{y} ; \boldsymbol{\theta})$ por $\boldsymbol{\Psi}_{m}(\boldsymbol{\theta})$.

1. A função de estimação $\boldsymbol{\Psi}_{m}(\boldsymbol{\theta})$ é uma função de estimação não viesada se

$$
\mathrm{E}_{\theta}\left\{\boldsymbol{\Psi}_{m}(\boldsymbol{\theta})\right\}=\mathbf{0} .
$$

2. Se todas as funções de estimação, $\boldsymbol{\psi}_{i}, i=1, \ldots, m$, são não viesadas, então a função de estimação $\boldsymbol{\Psi}_{m}$ baseada na amostra de tamanho $m$ também será não viesada.

3. Pode ser provado, sobre certas condições de regularidade que são definidas em Crowder (1987) e Godambe (1991), que a sequência de raízes $\left\{\hat{\boldsymbol{\theta}}_{m}\right\}_{m=1}^{\infty}$ associada com uma função de estimação regular (Godambe, 1991) $\boldsymbol{\Psi}_{m}(\boldsymbol{\theta})$, é tal que $\hat{\boldsymbol{\theta}}_{m} \stackrel{\mathcal{P}}{\rightarrow} \boldsymbol{\theta}$. Sob essas condições,

$$
\sqrt{m}\left(\hat{\boldsymbol{\theta}}_{m}-\boldsymbol{\theta}\right) \stackrel{\mathcal{D}}{\rightarrow} N_{p}\left(\mathbf{0}, \mathbf{J}_{\mathbf{\Psi}}^{-1}(\boldsymbol{\theta})\right),
$$

em que

$$
\begin{aligned}
\mathbf{J}_{\Psi}(\boldsymbol{\theta}) & =\left\{\lim _{m \longrightarrow \infty} \mathbf{S}_{m}^{\top}(\boldsymbol{\theta})\right\}\left\{\lim _{m \longrightarrow \infty} \mathbf{V}_{m}(\boldsymbol{\theta})\right\}^{-1}\left\{\lim _{m \longrightarrow \infty} \mathbf{S}_{m}(\boldsymbol{\theta})\right\} \\
& =\left\{\lim _{m \longrightarrow \infty} \mathbf{S}_{m}^{\top}(\boldsymbol{\theta})\right\}\left\{\lim _{m \longrightarrow \infty} \mathbf{V}_{m}^{-1}(\boldsymbol{\theta})\right\}\left\{\lim _{m} \mathbf{S}_{m}(\boldsymbol{\theta})\right\} \\
& =\left\{\lim _{m \longrightarrow \infty} \mathbf{S}_{m}^{\top}(\boldsymbol{\theta}) \mathbf{V}_{m}^{-1}(\boldsymbol{\theta}) \mathbf{S}_{m}(\boldsymbol{\theta})\right\}
\end{aligned}
$$

desempenha o papel de uma matriz de informação de Godambe limite associada a $\boldsymbol{\Psi}_{m}$, com

$$
\mathbf{S}_{m}(\boldsymbol{\theta})=\sum_{i=1}^{m} \frac{\mathbf{S}_{\boldsymbol{\psi}_{i}}(\boldsymbol{\theta})}{m}, \quad \mathbf{S}_{\boldsymbol{\psi}_{i}}(\boldsymbol{\theta})=\mathrm{E}\left\{\frac{\partial}{\partial \boldsymbol{\theta}^{\top}} \boldsymbol{\psi}_{i}\left(y_{i} ; \boldsymbol{\theta}\right)\right\},
$$

sendo a matriz de sensibilidade e

$$
\mathbf{V}_{m}(\boldsymbol{\theta})=\sum_{i=1}^{m} \frac{\mathbf{V}_{\boldsymbol{\psi}_{i}}(\boldsymbol{\theta})}{m}, \quad \mathbf{V}_{\boldsymbol{\psi}_{i}}(\boldsymbol{\theta})=\mathrm{E}\left\{\boldsymbol{\Psi}_{m}\left(y_{i} ; \boldsymbol{\theta}\right) \mathbf{\Psi}_{m}^{\top}\left(y_{i} ; \boldsymbol{\theta}\right)\right\},
$$

a matriz de variabilidade.

4. Outro conceito importante desenvolvido por Godambe (1960) é o de otimilidade de uma função de estimação regular. Dizemos que uma função de estimação associada com o parâmetro $\boldsymbol{\theta}, \mathbf{\Psi}_{m}^{*}(\boldsymbol{\theta})$, é ótima se $\mathbf{J}_{\mathbf{\Psi}}^{-1}(\boldsymbol{\theta})-\mathbf{J}_{\mathbf{\Psi}^{*}}^{-1}(\boldsymbol{\theta})$ é definida não negativa para todas as funções de estimação $\boldsymbol{\Psi}_{m}$.

5. Sejam $\mathbf{Q}_{i}$ matrizes não estocásticas que podem depender do parâmetro de interesse e $\mathbf{u}_{i}=$ $\mathbf{u}_{i}\left(\mathbf{y}_{i} ; \boldsymbol{\theta}\right)$ vetores com média zero mutuamente independentes, $i=1, \ldots, m$, que dependem dos dados e do parâmetro de interesse. A classe das funçôes de estimação geradas por $\mathbf{u}_{i}$ é definida por

$$
\mathcal{L}(\mathbf{u})=\left\{\boldsymbol{\Psi}_{m}(\boldsymbol{\theta}) \in \mathcal{R}: \mathbf{\Psi}_{m}(\boldsymbol{\theta})=\sum_{i=1}^{m} \mathbf{Q}_{i}(\boldsymbol{\theta}) \mathbf{u}_{i}\left(\mathbf{y}_{i} ; \boldsymbol{\theta}\right)\right\},
$$


sendo que $\mathcal{R}$ contém todas as funções regulares de $\boldsymbol{\theta}$ e $\mathbf{u}=\left(\mathbf{u}_{1}^{\top}, \ldots, \mathbf{u}_{m}^{\top}\right)^{\top}$.

Crowder (1987) mostra que nessa classe $\mathcal{L}(\mathbf{u})$, a função de estimação ótima é dada por

$$
\mathbf{\Psi}_{m}^{*}(\boldsymbol{\theta})=\sum_{i=1}^{m} \mathbf{Q}_{i}^{*}(\boldsymbol{\theta}) \mathbf{u}_{i}\left(\mathbf{y}_{i} ; \boldsymbol{\theta}\right)
$$

em que

$$
\mathbf{Q}_{i}^{*}(\boldsymbol{\theta})=\mathrm{E}\left(\frac{\partial \mathbf{u}_{i}}{\partial \boldsymbol{\theta}^{\top}}\right)^{\top} \operatorname{Var}^{-1}\left(\mathbf{u}_{i}\right)
$$

\subsection{Equações de Estimação}

Agora que já falamos sobre funções de estimação, podemos começar a falar sobre equações de estimação, mas não antes de definir uma notação que será utilizada durante o texto. Seja $\mathbf{y}_{i}=\left(y_{i 1}, \ldots, y_{i n_{i}}\right)^{\top}$ um vetor $n_{i} \times 1$ de respostas para a $i$-ésima unidade experimental e $\mathbf{X}_{i}=$ $\left(\mathbf{x}_{i 1}, \ldots, \mathbf{x}_{i n_{i}}\right)^{\top}$ uma matriz $n_{i} \times p$ de valores de variáveis explicativas para a $i$-ésima unidade experimental, com $\mathbf{x}_{i j}=\left(x_{i j 1}, \ldots, x_{i j p}\right)^{\top}, i=1, \ldots, m$ e $j=1, \ldots, n_{i}$. Assumindo que a densidade marginal de $y_{i j}$ pertença à família exponencial, temos que

$$
f\left(y_{i j} ; \theta_{i j}, \phi\right)=\exp \left\{\phi\left[y_{i j} \theta_{i j}-b\left(\theta_{i j}\right)\right]+c\left(y_{i j}, \phi\right)\right\},
$$

sendo $\mathrm{E}\left(y_{i j}\right)=\mu_{i j}=b^{\prime}\left(\theta_{i j}\right), \operatorname{Var}\left(y_{i j}\right)=\phi^{-1} b^{\prime \prime}\left(\theta_{i j}\right)=\phi^{-1} a_{i j}, a_{i j}=\frac{d \mu_{i j}}{d \theta_{i j}}$ é a função de variância, $c(\cdot)$ uma função conhecida e $\phi^{-1}$ o parâmetro de dispersão conhecido. A função de variância $\left(a_{i j}\right)$ desempenha um papel importante na família exponencial, uma vez que a mesma caracteriza a distribuição.

As médias são modeladas como nos MLGs, ou seja,

$$
g\left(\mu_{i j}\right)=\eta_{i j}
$$

sendo $\eta_{i j}=\mathbf{x}_{i j}^{\top} \boldsymbol{\beta}$ o preditor linear, $\boldsymbol{\beta}=\left(\beta_{1}, \ldots, \beta_{p}\right)^{\top}, p<m$, o vetor de parâmetros desconhecidos a serem estimados e $g(\cdot)$ uma função de ligação.

Com a notação definida vamos verificar dois casos de equações de estimação. O que vai diferenciálos é a suposição de independência.

\subsubsection{Equações de Estimação Independentes}

Vamos primeiramente fazer um estudo das equações de estimação para um caso em que as observações repetidas de uma mesma unidade experimental são independentes.

Sejam $\mathbf{u}_{i}=\mathbf{y}_{i}-\boldsymbol{\mu}_{\boldsymbol{i}}$ vetores com média zero mutuamente independentes, com $\boldsymbol{\mu}_{i}=\left(\mu_{i 1}, \ldots, \mu_{i n_{i}}\right)^{\top}$ e $i=1, \ldots, m$. Assumindo independência, os termos que são necessários para definir a função de estimação ótima que foi determinada em (2.1) são dados por

$$
\mathrm{E}\left(\frac{\partial \mathbf{u}_{i}}{\partial \boldsymbol{\beta}^{\top}}\right)^{\top}=\mathrm{E}\left\{\frac{\partial}{\partial \boldsymbol{\beta}^{\top}}\left(\mathbf{y}_{i}-\boldsymbol{\mu}_{i}\right)\right\}^{\top}=-\left(\frac{\partial \boldsymbol{\mu}_{i}}{\partial \boldsymbol{\beta}^{\top}}\right)^{\top}=-\mathbf{X}_{i}^{\top} \mathbf{W}_{i}^{\frac{1}{2}} \mathbf{A}_{i}^{\frac{1}{2}}=-\mathbf{D}_{i}^{\top}
$$

e

$$
\operatorname{Var}\left(\mathbf{u}_{i}\right)=\operatorname{Var}\left(\mathbf{y}_{i}\right)=\operatorname{diag}\left\{\operatorname{Var}\left(y_{i j}\right)\right\}=\phi^{-1} \operatorname{diag}\left\{b^{\prime \prime}\left(\theta_{i j}\right)\right\}=\phi^{-1} \mathbf{A}_{i}
$$

sendo

$$
\begin{gathered}
\mathbf{X}_{i}=\frac{\partial \boldsymbol{\eta}_{i}}{\partial \boldsymbol{\beta}}=\left(\mathbf{x}_{i 1}, \ldots, \mathbf{x}_{i n_{i}}\right)^{\top}, \text { com dimensão } n_{i} \times p, \\
\mathbf{W}_{i}=\operatorname{diag}\left\{w_{i j}\right\}, w_{i j}=\frac{\left(\frac{d \mu_{i j}}{d \eta_{i j}}\right)^{2}}{a_{i j}}, \text { com dimensão } n_{i} \times n_{i} \mathrm{e}
\end{gathered}
$$




$$
\mathbf{A}_{i}=\operatorname{diag}\left\{\frac{d \mu_{i j}}{d \theta_{i j}}\right\}=\operatorname{diag}\left\{b^{\prime \prime}\left(\theta_{i j}\right)\right\}=\operatorname{diag}\left\{a_{i j}\right\}, \text { com dimensão } n_{i} \times n_{i},
$$

sendo $\boldsymbol{\eta}_{i}=\left(\eta_{i 1}, \ldots, \eta_{i n_{i}}\right)^{\top}$ e $j=1, \ldots, n_{i}$.

Notemos que $\mathbf{A}_{i}$ é uma matriz definida a partir da função de variância e $\mathbf{W}_{i}$ é uma matriz que depende da função de ligação utilizada na modelagem.

A função de estimação ótima obtida dos termos acima fica dada por

$$
\begin{aligned}
\boldsymbol{\Psi}_{m}^{*}(\boldsymbol{\beta}) & =\sum_{i=1}^{m} \mathrm{E}\left(\frac{\partial \mathbf{u}_{i}}{\partial \boldsymbol{\beta}^{\top}}\right)^{\top} \operatorname{Var}^{-1}\left(\mathbf{u}_{i}\right) \mathbf{u}_{i}\left(\mathbf{y}_{i} ; \boldsymbol{\beta}\right) \\
& =\sum_{i=1}^{m}\left(-\mathbf{D}_{i}^{\top}\right)\left(\phi^{-1} \mathbf{A}_{i}\right)^{-1}\left(\mathbf{y}_{i}-\boldsymbol{\mu}_{i}\right) \\
& =\phi \sum_{i=1}^{m} \mathbf{D}_{i}^{\top} \mathbf{A}_{i}^{-1}\left(\mathbf{y}_{i}-\boldsymbol{\mu}_{i}\right)
\end{aligned}
$$

que coincide com a função escore de um MLG. Esse resultado era esperado, uma vez que estamos considerando a suposição de independência entre as observações de uma mesma unidade experimental.

A equação obtida ao igualarmos uma função de estimação a zero é denominada Equação de Estimação. Logo, as Equaçôes de Estimação Independentes (EEIs) de $\boldsymbol{\beta}$ ficam dadas por

$$
\mathbf{\Psi}_{m I}^{*}\left(\hat{\boldsymbol{\beta}}_{I}^{*}\right)=\phi \sum_{i=1}^{m} \hat{\mathbf{D}}_{i}^{\top} \hat{\mathbf{A}}_{i}^{-1}\left(\mathbf{y}_{i}-\hat{\boldsymbol{\mu}}_{i}\right)=\mathbf{0} .
$$

Como já foi dito para as funções de estimação, sob condições gerais de regularidade, podemos demonstrar que a raiz da EEIs, $\hat{\boldsymbol{\beta}}_{I}^{*}$, é um estimador consistente de $\boldsymbol{\beta}$ tal que

$$
\sqrt{m}\left(\hat{\boldsymbol{\beta}}_{I}^{*}-\boldsymbol{\beta}\right) \stackrel{\mathcal{D}}{\rightarrow} \mathrm{N}_{p}\left(\mathbf{0},\left(\mathbf{J}_{I}^{*}\right)^{-1}\right)
$$

com

$$
\mathbf{J}_{I}^{*}=\lim _{m \longrightarrow \infty} \frac{\mathbf{J}_{m I}^{*}}{m},
$$

sendo $\mathbf{J}_{m I}^{*}$ a matriz de informação de Godambe de $\boldsymbol{\beta}$ associada a $\boldsymbol{\Psi}_{m I}^{*}$, dada por

$$
\mathbf{J}_{m I}^{*}=\left\{\sum_{i=1}^{m} \mathbf{S}_{i}\right\}\left\{\sum_{i=1}^{m} \mathbf{V}_{i}\right\}^{-1}\left\{\sum_{i=1}^{m} \mathbf{S}_{i}\right\}=\phi \sum_{i=1}^{m} \mathbf{D}_{i}^{\top} \mathbf{A}_{i}^{-1} \mathbf{D}_{i},
$$

pois

$$
\mathbf{S}_{i}=\mathrm{E}_{\boldsymbol{\beta}}\left\{\frac{\partial}{\partial \boldsymbol{\beta}^{\top}} \mathbf{D}_{i}^{\top} \mathbf{A}_{i}^{-1}\left(\mathbf{y}_{i}-\boldsymbol{\mu}_{i}\right)\right\}=\mathbf{D}_{i}^{\top} \mathbf{A}_{i}^{-1} \mathrm{E}_{\boldsymbol{\beta}}\left\{\frac{\partial}{\partial \boldsymbol{\beta}^{\top}}\left(\mathbf{y}_{i}-\boldsymbol{\mu}_{i}\right)\right\}=-\mathbf{D}_{i}^{\top} \mathbf{A}_{i}^{-1} \mathbf{D}_{i}
$$

$\mathrm{e}$

$$
\begin{aligned}
\mathbf{V}_{i} & =\mathrm{E}_{\boldsymbol{\beta}}\left[\mathbf{D}_{i}^{\top} \mathbf{A}_{i}^{-1}\left(\mathbf{y}_{i}-\boldsymbol{\mu}_{i}\right)\left(\mathbf{y}_{i}-\boldsymbol{\mu}_{i}\right)^{\top} \mathbf{A}_{i}^{-1} \mathbf{D}_{i}\right] \\
& =\mathbf{D}_{i}^{\top} \mathbf{A}_{i}^{-1} \operatorname{Var}\left(\mathbf{y}_{i}\right) \mathbf{A}_{i}^{-1} \mathbf{D}_{i} \\
& =\phi^{-1} \mathbf{D}_{i}^{\top} \mathbf{A}_{i}^{-1} \mathbf{A}_{i} \mathbf{A}_{i}^{-1} \mathbf{D}_{i} \\
& =-\phi^{-1} \mathbf{S}_{i} .
\end{aligned}
$$

Um estimador consistente para a matriz de variância-covariância de $\hat{\boldsymbol{\beta}}_{I}^{*}$, quando as observações de 
uma mesma unidade experimental são independentes fica dado por

$$
\left(\hat{\mathbf{J}}_{m I}^{*}\right)^{-1}=\left\{\phi \sum_{i=1}^{m} \hat{\mathbf{D}}_{i}^{\top} \hat{\mathbf{A}}_{i}^{-1} \hat{\mathbf{D}}_{i}\right\}^{-1}
$$

sendo todas as quantidades avaliadas em $\hat{\boldsymbol{\beta}}_{I}^{*}$. Notemos que, como $\boldsymbol{\Psi}_{m I}^{*}(\boldsymbol{\beta})$ é uma função escore, a matriz de informação de Godambe de $\boldsymbol{\beta}$ é igual à matriz de informação de Fisher de $\boldsymbol{\beta}$. Logo, vimos que equações de estimação independentes nada mais são do que uma forma de demonstrar os resultados de um MLG.

\subsubsection{Equações de Estimação Generalizadas}

A diferença agora é que nesta subseção vamos desenvolver o conceito de equações de estimação, mas para o caso em que é considerado que há uma dependência entre as observações repetidas em uma mesma unidade experimental.

Para obtermos as Equações de Estimação Generalizadas (EEGs), introduziremos algumas modificações em $\boldsymbol{\Psi}_{m I}^{*}(\cdot)$ dada em (2.2) de modo que a nova função de estimação incorpore alguma informação sobre a estrutura de dependência dos dados. Os termos da função de estimação ótima definidos em (2.1) são dados por

$$
\mathrm{E}\left(\frac{\partial \mathbf{u}_{i}}{\partial \boldsymbol{\beta}^{\top}}\right)^{\top}=\mathrm{E}\left\{\frac{\partial}{\partial \boldsymbol{\beta}^{\top}}\left(\mathbf{y}_{i}-\mathbf{u}_{i}\right)\right\}^{\top}=\left(\frac{\partial \boldsymbol{\mu}_{i}}{\partial \boldsymbol{\beta}^{\top}}\right)^{\top}=-\mathbf{X}_{i}^{\top} \mathbf{W}_{i}^{\frac{1}{2}} \mathbf{A}_{i}^{\frac{1}{2}}=-\mathbf{D}_{i}^{\top}
$$

e

$$
\operatorname{Var}\left(\mathbf{u}_{i}\right)=\operatorname{Var}\left(\mathbf{y}_{i}\right)=\operatorname{diag}\left\{\operatorname{Var}\left(y_{i j}\right)^{\frac{1}{2}}\right\} \mathbf{R}_{i} \operatorname{diag}\left\{\operatorname{Var}\left(y_{i j}\right)^{\frac{1}{2}}\right\}=\phi^{-1} \mathbf{A}_{i}^{\frac{1}{2}} \mathbf{R}_{i} \mathbf{A}_{i}^{\frac{1}{2}}=\boldsymbol{\Sigma}_{i},
$$

sendo $\mathbf{R}_{i}$ a verdadeira matriz de correlação de $\mathbf{y}_{i}, i=1, \ldots, m$, e todas as outras matrizes são iguais às que foram definidas para Equações de Estimação Independentes.

Logo, as equações de estimação de $\boldsymbol{\beta}$ quando consideramos a verdadeira matriz de correlação é equivalente ao seguinte:

$$
\mathbf{\Psi}_{m G}^{*}\left(\hat{\boldsymbol{\beta}}_{G}^{*}\right)=\sum_{i=1}^{m} \hat{\mathbf{D}}_{i}^{\top} \hat{\boldsymbol{\Sigma}}_{i}^{-1}\left(\mathbf{y}_{i}-\hat{\boldsymbol{\mu}}_{i}\right)=\mathbf{0} .
$$

Sob condições gerais de regularidade, é possível mostrar que $\hat{\boldsymbol{\beta}}_{G}^{*}$, raiz de (2.3), é um estimador consistente de $\boldsymbol{\beta}$ e ainda temos que

$$
\sqrt{m}\left(\hat{\boldsymbol{\beta}}_{G}^{*}-\boldsymbol{\beta}\right) \stackrel{\mathcal{D}}{\rightarrow} \mathrm{N}_{p}\left(\mathbf{0},\left(\mathbf{J}_{G}^{*}\right)^{-1}\right),
$$

com

$$
\mathbf{J}_{G}^{*}=\lim _{m \longrightarrow \infty} \frac{\mathbf{J}_{m G}^{*}}{m},
$$

sendo $\mathbf{J}_{m G}^{*}$ a matriz de informação de Godambe de $\boldsymbol{\beta}$ associada a $\mathbf{\Psi}_{m G}^{*}$, dada por

$$
\mathbf{J}_{m G}^{*}=\left\{\sum_{i=1}^{m} \mathbf{S}_{i}\right\}\left\{\sum_{i=1}^{m} \mathbf{V}_{i}\right\}^{-1}\left\{\sum_{i=1}^{m} \mathbf{S}_{i}\right\}=\sum_{i=1}^{m} \mathbf{D}_{i}^{\top} \boldsymbol{\Sigma}_{i}^{-1} \mathbf{D}_{i},
$$

pois

$$
\mathbf{S}_{i}=\mathrm{E}_{\boldsymbol{\beta}}\left\{\frac{\partial}{\partial \boldsymbol{\beta}^{\top}} \mathbf{D}_{i}^{\top} \boldsymbol{\Sigma}_{i}^{-1}\left(\mathbf{y}_{i}-\boldsymbol{\mu}_{i}\right)\right\}=\mathbf{D}_{i}^{\top} \boldsymbol{\Sigma}_{i}^{-1} \mathrm{E}_{\boldsymbol{\beta}}\left\{\frac{\partial}{\partial \boldsymbol{\beta}^{\top}}\left(\mathbf{y}_{i}-\boldsymbol{\mu}_{i}\right)\right\}=-\mathbf{D}_{i}^{\top} \boldsymbol{\Sigma}_{i}^{-1} \mathbf{D}_{i}
$$


$\mathrm{e}$

$$
\begin{aligned}
\mathbf{V}_{i} & =\mathrm{E}_{\boldsymbol{\beta}}\left\{\mathbf{D}_{i}^{\top} \boldsymbol{\Sigma}_{i}^{-1}\left(\mathbf{y}_{i}-\boldsymbol{\mu}_{i}\right)\left(\mathbf{y}_{i}-\boldsymbol{\mu}_{i}\right)^{\top} \boldsymbol{\Sigma}_{i}^{-1} \mathbf{D}_{i}\right\} \\
& =\mathbf{D}_{i}^{\top} \boldsymbol{\Sigma}_{i}^{-1} \operatorname{Var}\left(\mathbf{y}_{i}\right) \boldsymbol{\Sigma}_{i}^{-1} \mathbf{D}_{i} \\
& =\mathbf{D}_{i}^{\top} \boldsymbol{\Sigma}_{i}^{-1} \mathbf{D}_{i} \\
& =-\mathbf{S}_{i} .
\end{aligned}
$$

Neste caso, um estimador consistente para a matriz de variância-covariância de $\hat{\boldsymbol{\beta}}_{G}^{*}$ fica dado por

$$
\left(\hat{\mathbf{J}}_{m G}^{*}\right)^{-1}=\left\{\sum_{i=1}^{m} \hat{\mathbf{D}}_{i}^{\top} \hat{\boldsymbol{\Sigma}}_{i}^{-1} \hat{\mathbf{D}}_{i}\right\}^{-1},
$$

sendo todas as quantidades avaliadas em $\hat{\boldsymbol{\beta}}_{G}^{*}$. Na literatura, esse estimador recebe os nomes de estimador "naive" ou "model-based".

Na prática, a função $\Psi_{m G}^{*}(\cdot)$ é pouco utilizada, pois em geral, a verdadeira matriz de correlação, $\mathbf{R}_{i}$, é desconhecida. Para contornar esse problema, Liang e Zeger (1986) definiram $\mathbf{R}_{i}(\boldsymbol{\rho})$ como sendo uma matriz simétrica $n_{i} \times n_{i}$ satisfazendo às condições para ser uma matriz de correlação, denominada matriz trabalho em que $\boldsymbol{\rho}$, um vetor $s \times 1$, caracteriza completamente $\mathbf{R}_{i}(\boldsymbol{\rho})$. Como a matriz trabalho representa a correlação entre as observações de um mesmo grupo, ajustadas pelos valores das variáveis explicativas presentes no modelo, os valores que $\boldsymbol{\rho}$ pode assumir estão no intervalo $[-1 ; 1]$ e a dimensão dessa matriz é determinada pelo número de observações feitas em cada grupo.

O principal benefício da abordagem EEGs é que a matriz de correlação pode ser arbitrariamente parametrizada. As restrições estruturais dos valores que são substituídos nessa matriz alternativa definem os vários tipos de correlação que são geralmente encontradas em softwares.

Com isso, as Equaçôes de Estimação Generalizadas de $\boldsymbol{\beta}$ são dadas por

$$
\boldsymbol{\Psi}_{m}^{G}\left(\hat{\boldsymbol{\beta}}_{G}\right)=\Psi_{m}^{G}\left(\hat{\boldsymbol{\beta}}_{G}, \hat{\boldsymbol{\rho}}\left(\hat{\boldsymbol{\beta}}_{G}, \hat{\phi}\right)\right)=\sum_{i=1}^{m} \hat{\mathbf{D}}_{i}^{\top} \hat{\boldsymbol{\Omega}}_{i}^{-1}\left(\mathbf{y}_{i}-\hat{\boldsymbol{\mu}}_{i}\right)=\mathbf{0}
$$

em que

$$
\boldsymbol{\Omega}_{i}=\boldsymbol{\Omega}_{i}(\boldsymbol{\rho}, \phi)=\phi^{-1} \mathbf{A}_{i}^{\frac{1}{2}} \mathbf{R}_{i}(\boldsymbol{\rho}) \mathbf{A}_{i}^{\frac{1}{2}},
$$

com $\hat{\boldsymbol{\rho}}$ sendo um estimador consistente de $\boldsymbol{\rho}$.

As equações de estimação dadas em (2.5) deixam de ser ótimas e, por isso, podem ser viesadas. É necessário, então, que $\hat{\boldsymbol{\rho}}$ seja determinado de modo que o estimador de $\boldsymbol{\beta}$, obtido a partir (2.5), continue sendo consistente e assintoticamente normal.

Na prática, $\phi$ quase sempre é desconhecido. Logo, também é necessário propor um estimador para esse parâmetro.

Para preservar as propriedades que foram citadas para um estimador de $\boldsymbol{\beta}$ que provém de uma equação de estimação ótima, há algumas condições para os estimadores de $\boldsymbol{\rho}$ e $\phi$. O teorema que especifica essas condições pode ser encontrado facilmente na tese de Venezuela (2007) e a prova desse resultado encontra-se em Liang e Zeger (1986).

Sob as condições do teorema, temos que $\hat{\boldsymbol{\beta}}_{G}$ é um estimador consistente de $\boldsymbol{\beta}$ e

$$
\sqrt{m}\left(\hat{\boldsymbol{\beta}}_{G}-\boldsymbol{\beta}\right) \stackrel{\mathcal{D}}{\rightarrow} \mathrm{N}_{p}\left(\mathbf{0}, \mathbf{J}_{G}^{-1}\right),
$$

com

$$
\mathbf{J}_{G}=\lim _{m \longrightarrow \infty} \frac{\mathbf{J}_{m G}}{m},
$$


sendo $\mathbf{J}_{m G}$ a matriz de informação de Godambe de $\boldsymbol{\beta}$ associada a $\boldsymbol{\Psi}_{m G}$, dada por

$$
\mathbf{J}_{m G}=\left\{\sum_{i=1}^{m} \mathbf{S}_{i}\right\}\left\{\sum_{i=1}^{m} \mathbf{V}_{i}\right\}^{-1}\left\{\sum_{i=1}^{m} \mathbf{S}_{i}\right\}
$$

em que

$$
\mathbf{S}_{i}=\mathrm{E}_{\boldsymbol{\beta}}\left[\frac{\partial}{\partial \boldsymbol{\beta}^{\top}} \mathbf{D}_{i}^{\top} \boldsymbol{\Omega}_{i}^{-1}\left(\mathbf{y}_{i}-\boldsymbol{\mu}_{i}\right)\right]=\mathbf{D}_{i}^{\top} \boldsymbol{\Omega}_{i}^{-1} \mathrm{E}_{\boldsymbol{\beta}}\left[\frac{\partial}{\partial \boldsymbol{\beta}^{\top}}\left(\mathbf{y}_{i}-\boldsymbol{\mu}_{i}\right)\right]=-\mathbf{D}_{i}^{\top} \boldsymbol{\Omega}_{i}^{-1} \mathbf{D}_{i}
$$

e

$$
\begin{aligned}
\mathbf{V}_{i} & =\mathrm{E}_{\boldsymbol{\beta}}\left[\mathbf{D}_{i}^{\top} \boldsymbol{\Omega}_{i}^{-1}\left(\mathbf{y}_{i}-\boldsymbol{\mu}_{i}\right)\left(\mathbf{y}_{i}-\boldsymbol{\mu}_{i}\right)^{\top} \boldsymbol{\Omega}_{i}^{-1} \mathbf{D}_{i}\right] \\
& =\mathbf{D}_{i}^{\top} \boldsymbol{\Omega}_{i}^{-1} \operatorname{Var}\left(\mathbf{y}_{i}\right) \boldsymbol{\Omega}_{i}^{-1} \mathbf{D}_{i} .
\end{aligned}
$$

Vale observar que os resultados assintóticos do teorema são válidos mesmo quando $\mathbf{R}_{i}(\boldsymbol{\rho})$ não corresponde à verdadeira matriz de correlação de $\mathbf{y}_{i}$.

A matriz de variância - covariância de $\hat{\boldsymbol{\beta}}_{G}$ pode ser consistentemente estimada por

$$
\hat{\mathbf{J}}_{m G}^{-1}=\left\{\sum_{i=1}^{m} \hat{\mathbf{S}}_{i}\right\}^{-1}\left\{\sum_{i=1}^{m} \hat{\mathbf{D}}_{i}^{\top} \hat{\mathbf{\Omega}}_{i}^{-1} \hat{\mathbf{u}}_{i} \hat{\mathbf{u}}_{i}^{\top} \hat{\mathbf{\Omega}}_{i}^{-1} \hat{\mathbf{D}}_{i}\right\}\left\{\sum_{i=1}^{m} \hat{\mathbf{S}}_{i}\right\}^{-1} .
$$

A estimativa da expressão acima é encontrada substituindo $\boldsymbol{\beta}, \boldsymbol{\rho}$ e $\phi$ por suas respectivas estimativas consistentes. Na literatura esse estimador recebe os nomes de estimador robusto, empírico ou sanduíche. Quando $\mathbf{R}_{i}(\boldsymbol{\rho})$ for a verdadeira matriz de correlação dos $\mathbf{y}_{i}$ 's, então $\boldsymbol{\Omega}_{i}=\operatorname{Var}\left(\mathbf{y}_{i}\right)$ e o estimador robusto será igual ao estimador "naive"expresso em (2.4).

Considerando que o modelo de regressão está corretamente especificado, o estimador "naive"é consistente se a matriz de trabalho também está corretamente especificada. Já o estimador robusto é sempre consistente. Além disso, o estimador robusto é assintoticamente não viesado, mas pode ser altamente viesado quando o número de unidades experimentais $(m)$ for pequeno. Quando $m<20$ o estimador "naive"pode ter melhores propriedades (Prentice, 1988) mesmo se $\mathbf{R}_{i}(\boldsymbol{\rho})$ não estiver corretamente especificada.

Uma indicação da adequação de uma matriz $\mathbf{R}_{i}(\boldsymbol{\rho})$ ao modelo ocorre quando as duas estimativas, "naive"e robusta, são próximas (Johnston e Stokes, 1996).

\subsubsection{Estimação de $\beta, \rho$ e $\phi$}

O processo iterativo para obter $\hat{\boldsymbol{\beta}}_{G}$ combina o método escore de Fisher para estimar $\boldsymbol{\beta}$ com o método dos momentos para estimar $\boldsymbol{\rho}$ e $\phi$. Logo, expandindo as EEGs dadas na equação (2.5), o processo iterativo para estimar $\boldsymbol{\beta}$ fica dado por

$$
\begin{aligned}
\boldsymbol{\beta}_{G}^{(h+1)} & =\boldsymbol{\beta}_{G}^{(h)}-\left[\mathrm{E}\left\{\frac{\partial}{\partial \boldsymbol{\beta}^{\top}} \mathbf{\Psi}_{m}^{G}\left(\boldsymbol{\beta}_{G}^{(h)}\right)\right\}\right]^{-1} \mathbf{\Psi}_{m}^{G}\left(\boldsymbol{\beta}_{G}^{(h)}\right) \\
& =\boldsymbol{\beta}_{G}^{(h)}+\left\{\sum_{i=1}^{m} \mathbf{D}_{i}^{(h) \top}\left(\boldsymbol{\Omega}_{i}^{(h)}\right)^{-1} \mathbf{D}_{i}^{(h)}\right\}^{-1}\left\{\sum_{i=1}^{m} \mathbf{D}_{i}^{(h) \top}\left(\boldsymbol{\Omega}_{i}^{(h)}\right)^{-1}\left(\mathbf{y}_{i}-\boldsymbol{\mu}_{i}^{(h)}\right)\right\},
\end{aligned}
$$

sendo $h=0,1, \ldots$ o número de iterações. $\mathrm{O}$ índice $h$ no lado direito das equações acima indica que as matrizes e os vetores são atualizados pelas estimativas de $\boldsymbol{\beta}, \boldsymbol{\rho}$ e $\phi$ da $h$-ésima iteração.

Lembrando que a matriz $\mathbf{D}_{i}$ é dada pela multiplicação das matrizes $\mathbf{A}_{i}^{\frac{1}{2}} \mathbf{W}_{i}^{\frac{1}{2}} \mathbf{X}_{i}$, podemos rees- 
crever a expressão (2.6) da seguinte forma:

$$
\boldsymbol{\beta}_{G}^{(h+1)}=\left[\left\{\sum_{i=1}^{m} \mathbf{X}_{i}^{\top} \boldsymbol{\Delta}_{i} \mathbf{X}_{i}\right\}^{-1}\left\{\sum_{i=1}^{m} \mathbf{X}_{i}^{\top} \boldsymbol{\Delta}_{i}^{-1} \mathbf{z}_{i}\right\}\right]^{(h)},
$$

$\operatorname{com} \boldsymbol{\Delta}_{i}=\mathbf{W}_{i}^{\frac{1}{2}} \mathbf{A}_{i}^{\frac{1}{2}} \boldsymbol{\Omega}_{i}^{-1} \mathbf{A}_{i}^{\frac{1}{2}} \mathbf{W}_{i}^{\frac{1}{2}}$ e $\mathbf{z}_{i}=\boldsymbol{\eta}_{i}+\left(\mathbf{A}_{i}^{\frac{1}{2}} \mathbf{W}_{i}^{\frac{1}{2}}\right)^{-1}\left(\mathbf{y}_{i}-\boldsymbol{\mu}_{i}\right)$.

Notemos que chegamos a um processo iterativo de mínimos quadrados reponderados em que $\mathbf{z}$ desempenha o papel de uma variável dependente modificada e $\boldsymbol{\Delta}$ é uma matriz de pesos. Ambos mudam a cada passo do processo iterativo.

Liang e Zeger (1986) utilizam o método dos momentos para estimar os parâmetros de correlação $\boldsymbol{\rho}$ e o parâmetro de escala $\phi$, e os escrevem em função dos resíduos de Pearson. Com isso, para cada observação $y_{i j}$, o resíduo de Pearson obtido no passo $h$ fica dado por

$$
r_{i j}^{(h)}=\frac{y_{i j}-\mu_{i j}^{(h)}}{\sqrt{a_{i j}^{(h)}}},
$$

sendo $a_{i j}$ o $j$-ésimo elemento da diagonal principal de $\mathbf{A}_{i}$.

A estimativa de $\phi$ obtida no $h$-ésimo passo do processo iterativo fica dada por

$$
\phi^{(h)}=\left\{\sum_{i=1}^{m} \frac{\left(r_{i j}^{(h)}\right)^{2}}{(N-p)}\right\}^{-1},
$$

$\operatorname{com} N=\sum_{i=1}^{m} n_{i}$.

O procedimento das EEGs para estimar $\boldsymbol{\beta}$ permite que a estrutura de correlação entre as observações da mesma unidade experimental seja especificada de diferentes formas. A seguir, apresentamos algumas estruturas usadas para definir $\mathbf{R}_{i}(\boldsymbol{\rho})$. Notemos, entretanto, que para qualquer $\mathbf{R}_{i}(\boldsymbol{\rho})$ dada, $\hat{\boldsymbol{\beta}}_{G}$ e $\hat{\mathbf{J}}_{m G}^{-1}$ serão consistentes e a eficiência cresce quanto mais próxima da verdadeira matriz de correlação estiver a escolha da matriz de trabalho.

1. A matriz de correlação padrão simétrica assume que $\operatorname{Corr}\left(y_{i j}, y_{i l}\right)=\rho, \forall j \neq l$ e $1 \leq j, l \leq n_{i}$. Então, a estimativa de $\rho$ no passo $h$ fica dada por

$$
\rho^{(h)}=\frac{\phi^{(h)}}{m} \sum_{i=1}^{m} \frac{1}{n_{i}\left(n_{i}-1\right)} \sum_{j=1}^{n_{i}} \sum_{\substack{l=1 \\ l \neq j}}^{n_{i}} r_{i j}^{(h)} r_{i l}^{(h)} .
$$

2. A matriz de correlação autoregressiva de primeira ordem, AR-1, especifica que $\operatorname{Corr}\left(y_{i j}, y_{i l}\right)=$ $\rho^{|j-l|}, 1 \leq j, l \leq n_{i}$.

$$
\rho^{(h)}=\frac{\phi^{(h)}}{m} \sum_{i=1}^{m} \frac{1}{\left(n_{i}-1\right)} \sum_{j=1}^{\left(n_{i}-1\right)} r_{i j}^{(h)} r_{i(j+1)}^{(h)} .
$$

3. Quando a matriz de correlação é a não estruturada, o $\left(j, j^{\prime}\right)$-ésimo elemento de $\mathbf{R}_{i}$ pode ser estimado por

$$
R_{j j^{\prime}}=\frac{\phi^{(h)}}{m}\left\{\sum_{i=1}^{m} r_{i j}^{(h)} r_{i\left(j^{\prime}\right)}^{(h)}\right\} .
$$

Agora que temos as fórmulas para estimar os parâmetros, vamos apresentar as etapas que mostram na prática como é possível estimar os parâmetros $\boldsymbol{\beta}, \boldsymbol{\rho}$ e $\phi$. 


\section{Etapas para a estimação de $\beta, \rho$ e $\phi$}

1. Supondo independência entre as observações da mesma unidade experimental, utilizamos a equação (2.8) para estimar $\boldsymbol{\beta}$. O processo iterativo pode ser iniciado considerando $\mu_{i j}^{(0)}=y_{i j}$. É possível notar que a suposição de independência elimina os parâmetros $\boldsymbol{\rho}$ e $\phi$ do processo de estimação de $\boldsymbol{\beta}$ neste primeiro passo.

2. Definimos uma estrutura de correlação de trabalho para ser utilizada na modelagem dos dados. Se a estrutura for a independente, a estimativa de $\boldsymbol{\beta}$ é o vetor de valores obtidos na convergência do processo iterativo da etapa 1. Caso contrário, passamos para o próximo passo.

3. Utilizamos novamente a equação (2.8), considerando como $\boldsymbol{\beta}_{G}^{(0)}$ a estimativa de $\boldsymbol{\beta}$ encontrada no passo 1.

4. Calculamos o resíduo de Pearson $\left(r_{i j}^{(0)}\right)$, o parâmetro de escala $\left(\phi^{(0)}\right)$ e os parâmetros de correlação $\left(\boldsymbol{\rho}^{(0)}\right)$.

5. No passo seguinte do processo iterativo, calculamos os parâmetros de regressão $\left(\boldsymbol{\beta}_{G}^{(h)}\right)$ através da expressão (2.8).

6. Em seguida o resíduo de Pearson $\left(r_{i j}^{(h)}\right)$ é calculado através de $(2.9)$, o parâmetro de escala $\left(\phi^{(h)}\right)$ através de $(2.10)$ e os parâmetros de correlação $\left(\boldsymbol{\rho}^{(h)}\right)$ através das formas apresentadas de (2.2.3). E esse passo do processo é repetido até a convergência.

\subsection{Exemplo}

Para exemplificarmos uma forma de encontrar as Equações de Estimação Generalizadas para um caso particular, vamos utilizar a distribuição de contagem Poisson, pois está de acordo com a suposição de que a distribuição marginal de $y_{i j}$ pertence à família exponencial. E vamos utilizar neste exemplo a função de ligação canônica e matriz simétrica para matriz trabalho. Considerando as seguintes suposições:

(i) $\mathbf{y}_{i}=\left(y_{i 1}, \ldots, y_{i r_{i}}\right)^{\top}$, com $y_{i j} \sim \operatorname{Poisson}\left(\mu_{i}\right), i=1, \ldots, m$ e $j=1, \ldots, n_{i}$;

(ii) $\log \left(\mu_{i}\right)=\mathbf{x}_{i}^{\top} \boldsymbol{\beta}=\eta_{i}, \operatorname{com} \mathbf{x}_{i}=\left(x_{i 1}, \ldots, x_{i p}\right)^{\top}$ e $\boldsymbol{\beta}=\left(\beta_{1}, \ldots, \beta_{p}\right)^{\top}$;

(iii) $\operatorname{Corr}\left(y_{i j}, y_{i j^{\prime}}\right)=\left\{\begin{array}{ll}1, & j=j^{\prime} \\ \rho, & j \neq j^{\prime}\end{array}\right.$.

Como sabemos que $y_{i j}$ tem distribuição Poisson, que pertence à família exponencial, podemos reescrever a sua função de probabilidades como

$$
f\left(y_{i j} ; \theta_{i j}, \phi\right)=\exp \left\{y_{i j} \log \mu_{i}-\mu_{i}\right\}-\frac{1}{y_{i j} !},
$$

em que $\phi=1, \mathrm{E}\left(y_{i j}\right)=\mu_{i}$ e $\operatorname{Var}\left(y_{i j}\right)=V_{i j}=\mu_{i}$.

As Equações de Estimação Generalizadas como foram definidas em (2.5) podem ser encontradas então da seguinte forma:

$$
\boldsymbol{\Psi}_{\boldsymbol{\beta}}=\boldsymbol{\Psi}_{m}^{G}(\boldsymbol{\beta})=\sum_{i=1}^{m} \mathbf{D}_{i}^{\top} \boldsymbol{\Omega}_{i}^{-1}(\rho)\left(\mathbf{y}_{-} \boldsymbol{\mu}_{\boldsymbol{i}}\right) .
$$


Sabemos que $\boldsymbol{\Omega}_{i}(\rho)=\mathbf{A}_{i}^{\frac{1}{2}} \mathbf{R}_{i}(\rho) \mathbf{A}_{i}^{\frac{1}{2}}$, já que para o nosso exemplo $\phi=1$. Além disso, sabemos que

$$
\mathbf{A}_{i}=\operatorname{diag}\left\{a_{i j}\right\}=\operatorname{diag}\left\{\mu_{i}\right\}=\mu_{i} \mathbf{I}_{n_{i}}, \quad \mathbf{R}_{i}(\rho)=\left[\begin{array}{cccc}
1 & \rho & \ldots & \rho \\
\rho & 1 & \ldots & \rho \\
\vdots & \vdots & \ddots & \vdots \\
\rho & \rho & \ldots & 1
\end{array}\right] .
$$

Portanto, quando queremos a inversa temos $\boldsymbol{\Omega}_{i}^{-1}(\rho)=\mathbf{A}_{i}^{\frac{-1}{2}} \mathbf{R}_{i}^{-1}(\rho) \mathbf{A}_{i}^{\frac{-1}{2}}$, com

$$
\mathbf{R}_{i}^{-1}(\rho)=\frac{1}{1-\rho}\left[\mathbf{I}_{n_{i}}-\frac{\rho}{1+\left(n_{i}-1\right) \rho} \mathbf{J}_{n_{i}}\right], \quad \mathbf{I}_{n_{i}}=\left[\begin{array}{cccc}
1 & 0 & \ldots & 0 \\
0 & 1 & \ldots & 0 \\
\vdots & \vdots & \ddots & \vdots \\
0 & 0 & \ldots & 1
\end{array}\right], \quad \mathbf{J}_{n_{i}}=\left[\begin{array}{cccc}
1 & 1 & \ldots & 1 \\
1 & 1 & \ldots & 1 \\
\vdots & \vdots & \ddots & \vdots \\
1 & 1 & \ldots & 1
\end{array}\right]
$$

Portanto, podemos escrever

$$
\boldsymbol{\Omega}_{i}^{-1}(\rho)=\mathbf{A}_{i}^{\frac{-1}{2}} \mathbf{R}_{i}^{-1}(\rho) \mathbf{A}_{i}^{\frac{-1}{2}}=\frac{1}{\sqrt{\mu_{i}}} \mathbf{I}_{r_{i}} \mathbf{R}_{i}^{-1}(\rho) \frac{1}{\sqrt{\mu_{i}}} \mathbf{I}_{r_{i}}=\frac{1}{\mu_{i}} \mathbf{R}_{i}^{-1}(\rho) .
$$

Além disso, sabemos que $\mathbf{D}_{i}^{\top}=\mathbf{X}_{i}^{\top} \mathbf{W}_{i}^{\frac{1}{2}} \mathbf{A}_{i}^{\frac{1}{2}}$. Encontrando $\mathbf{W}_{i}$ tal que

$$
w_{i j}=\frac{\left(e^{\eta_{i}}\right)^{2}}{V_{i j}}=\frac{\mu_{i}^{2}}{\mu_{i}}=\mu_{i}, \quad \mathbf{W}_{i}=\operatorname{diag}\left\{w_{i j}\right\}=\operatorname{diag}\left\{\mu_{i}\right\}=\mu_{i} \mathbf{I}_{r_{i}} .
$$

Logo

$$
\mathbf{D}_{i}^{\top}=\mathbf{X}_{i}^{\top} \mathbf{W}_{i}^{\frac{1}{2}} \mathbf{A}_{i}^{\frac{1}{2}}=\mathbf{X}_{i}^{\top} \sqrt{\mu_{i}} \mathbf{I}_{r_{i}} \sqrt{\mu_{i}} \mathbf{I}_{r_{i}}=\mu_{i} \mathbf{X}_{i}^{\top} .
$$

Retornando na EEG, obtemos

$$
\begin{aligned}
& \boldsymbol{\Psi}_{\boldsymbol{\beta}}=\sum_{i=1}^{m} \mathbf{D}_{i}^{\top} \boldsymbol{\Omega}_{i}^{-1}(\rho)\left(\mathbf{y}_{-} \boldsymbol{\mu}_{\boldsymbol{i}}\right) .=\sum_{i=1}^{m} \mu_{i} \mathbf{X}_{i}^{\top} \frac{1}{\mu_{i}} \mathbf{R}_{i}^{-1}(\rho)\left(\mathbf{y}_{-} \boldsymbol{\mu}_{\boldsymbol{i}}\right)=\sum_{i=1}^{m} \mathbf{X}_{i}^{\top} \mathbf{R}_{i}^{-1}(\rho)\left(\mathbf{y}_{-} \boldsymbol{\mu}_{\boldsymbol{i}}\right), \\
& \mathbf{X}_{i}^{\top} \mathbf{R}_{i}^{-1}(\rho)=\left[\begin{array}{cccc}
x_{i 1} & x_{i 1} & \ldots & x_{i 1} \\
x_{i 2} & x_{i 2} & \ldots & x_{i 2} \\
\vdots & \vdots & \ddots & \vdots \\
x_{i p} & x_{i p} & \ldots & x_{i p}
\end{array}\right] \frac{1}{(1-\rho)}\left[\begin{array}{cccc}
1-\frac{\rho}{1+\left(n_{i}-1\right) \rho} & -\frac{\rho}{1+\left(n_{i}-1\right) \rho} & \ldots & -\frac{\rho}{1+\left(n_{i}-1\right) \rho} \\
-\frac{\rho}{1+\left(n_{i}-1\right) \rho} & 1-\frac{\rho}{1+\left(n_{i}-1\right) \rho} & \ldots & -\frac{\rho}{1+\left(n_{i}-1\right) \rho} \\
\vdots & \vdots & \ddots & \vdots \\
-\frac{\rho}{1+\left(n_{i}-1\right) \rho} & -\frac{\rho}{1+\left(n_{i}-1\right) \rho} & \ldots & 1-\frac{\rho}{1+\left(n_{i}-1\right) \rho}
\end{array}\right] \\
& =\frac{1}{(1-\rho)}\left[\begin{array}{cccc}
x_{i 1}\left(1-\frac{n_{i} \rho}{1+\left(n_{i}-1\right) \rho}\right) & x_{i 1}\left(1-\frac{n_{i} \rho}{1+\left(n_{i}-1\right) \rho}\right) & \ldots & x_{i 1}\left(1-\frac{n_{i} \rho}{1+\left(n_{i}-1\right) \rho}\right) \\
x_{i 2}\left(1-\frac{n_{i} \rho}{1+\left(n_{i}-1\right) \rho}\right) & x_{i 2}\left(1-\frac{n_{i} \rho}{1+\left(n_{i}-1\right) \rho}\right) & \ldots & x_{i 2}\left(1-\frac{n_{i} \rho}{1+\left(n_{i}-1\right) \rho}\right) \\
\vdots & \vdots & \ddots & \vdots \\
x_{i p}\left(1-\frac{n_{i} \rho}{1+\left(n_{i}-1\right) \rho}\right) & x_{i p}\left(1-\frac{n_{i} \rho}{1+\left(n_{i}-1\right) \rho}\right) & \ldots & x_{i p}\left(1-\frac{n_{i} \rho}{1+\left(n_{i}-1\right) \rho}\right)
\end{array}\right] \\
& =\frac{1}{(1-\rho)}\left[\begin{array}{cccc}
x_{i 1}\left(\frac{1-\rho}{1+\left(n_{i}-1\right) \rho}\right) & x_{i 1}\left(\frac{1-\rho}{1+\left(n_{i}-1\right) \rho}\right) & \ldots & x_{i 1}\left(\frac{1-\rho}{1+\left(n_{i}-1\right) \rho}\right) \\
x_{i 2}\left(\frac{1-\rho}{1+\left(n_{i}-1\right) \rho}\right) & x_{i 2}\left(\frac{1-\rho}{1+\left(n_{i}-1\right) \rho}\right) & \ldots & x_{i 2}\left(\frac{1-\rho}{1+\left(n_{i}-1\right) \rho}\right) \\
\vdots & \vdots & \ddots & \vdots \\
x_{i p}\left(\frac{1-\rho}{1+\left(n_{i}-1\right) \rho}\right) & x_{i p}\left(\frac{1-\rho}{1+\left(n_{i}-1\right) \rho}\right) & \ldots & x_{i p}\left(\frac{1-\rho}{1+\left(n_{i}-1\right) \rho}\right)
\end{array}\right]
\end{aligned}
$$




$$
\begin{aligned}
\mathbf{X}_{i}^{\top} \mathbf{R}_{i}^{-1}(\rho) & =\frac{1}{(1-\rho)} \frac{(1-\rho)}{1+\left(n_{i}-1\right) \rho}\left[\begin{array}{cccc}
x_{i 1} & x_{i 1} & \ldots & x_{i 1} \\
x_{i 2} & x_{i 2} & \ldots & x_{i 2} \\
\vdots & \vdots & \ddots & \vdots \\
x_{i p} & x_{i p} & \ldots & x_{i p}
\end{array}\right] \\
& =\frac{1}{1+\left(n_{i}-1\right) \rho} \mathbf{X}_{i}^{\top},
\end{aligned}
$$

e daí segue que

$$
\begin{aligned}
& \mathbf{X}_{i}^{\top} \mathbf{R}_{i}^{-1}(\rho)\left(\mathbf{y}-\boldsymbol{\mu}_{\boldsymbol{i}}\right)=\frac{1}{1+\left(n_{i}-1\right) \rho} {\left[\begin{array}{cccc}
x_{i 1} & x_{i 1} & \ldots & x_{i 1} \\
x_{i 2} & x_{i 2} & \ldots & x_{i 2} \\
\vdots & \vdots & \ddots & \vdots \\
x_{i p} & x_{i p} & \ldots & x_{i p}
\end{array}\right]\left[\begin{array}{c}
y_{i 1}-\mu_{i} \\
y_{i 2}-\mu_{i} \\
\vdots \\
y_{i n_{i}}-\mu_{i}
\end{array}\right] } \\
&=\frac{1}{1+\left(n_{i}-1\right) \rho}\left[\begin{array}{c}
x_{i 1}\left(\sum_{j=1}^{n_{i}} y_{i j}-\mu_{i}\right) \\
x_{i 2}\left(\sum_{j=1}^{n_{i}} y_{i j}-\mu_{i}\right) \\
\vdots \\
x_{i p}\left(\sum_{j=1}^{n_{i}} y_{i j}-\mu_{i}\right)
\end{array}\right] .
\end{aligned}
$$

Então, para um $\beta_{t}$ qualquer, obtemos

$$
\begin{aligned}
\mathbf{\Psi}_{\beta_{t}} & =\sum_{i=1}^{m} \frac{x_{i t}}{1+\left(n_{i}-1\right) \rho} \sum_{j=1}^{n_{i}}\left(y_{i j}-\mu_{i}\right) \\
& =\sum_{i=1}^{m} \frac{x_{i t}}{1+\left(n_{i}-1\right) \rho}\left(y_{i+}-n_{i} \mu_{i}\right), \text { com } y_{i+}=\sum_{j=1}^{n_{i}} y_{i j}
\end{aligned}
$$

\subsection{Métodos de Diagnóstico}

Como em toda modelagem, uma fase importante para a decisão do melhor modelo, consiste em aplicar técnicas de verificação de adequação do ajuste, ou seja, um diagnóstico do modelo. Essa etapa ajuda a encontrar possíveis discrepâncias que auxiliem na escolha do modelo que melhor se ajuste aos dados, levando em conta as suposições feitas.

Paula (2013) já tem bem definido algumas técnicas de diagnóstico para MLGs, e Venezuela (2003) também define essas técnicas para os modelos com dados de painel, ou seja, com medidas repetidas (vide também Venezuela et al. , 2007; Venezuela et al. , 2011). Outros trabalhos que são citados na literatura e que tratam de técnicas de diagnóstico para EEGs, por exemplo, são Chang (2000), que apresenta um teste não paramétrico para avaliar a aleatoriedade dos resíduos, Pan (2001) que apresenta medidas para escolher a melhor matriz de correlação e seleção de variáveis baseados no AIC (Critério de Informação de Akaike) e Preisser e Qaqish (1996) que apresentam formas de detectar observações e/ou unidades experimentais influentes em MLGs com medidas repetidas.

Para esse estudo vamos apresentar somente algumas das técnicas apresentadas e desenvolvidas em Venezuela (2003).

\section{- Pontos de Alavanca}

No contexto de EEGs há duas propostas para detectar pontos de alavanca, ou seja, pontos com 
influência desproporcional nos valores ajustados. A primeira proposta é avaliar se há pontos influentes nos valores ajustados de cada unidade experimental. Por exemplo, para avaliar a influência das observações nos valores da $i$-ésima unidade experimental é recomendado considerar o gráfico de indíces de $\hat{h}_{i j j}$, em que $\hat{h}_{i j j}$ denota o $j$-ésimo elemento da diagonal principal da matriz $\hat{\mathbf{H}}_{i}$, em que

$$
\hat{\mathbf{H}}=\operatorname{diag}\left(\hat{\mathbf{H}}_{1}, \ldots, \hat{\mathbf{H}}_{m}\right) \quad \text { e } \quad \hat{\mathbf{H}}_{i}=\hat{\boldsymbol{\Delta}}_{i}^{\frac{1}{2}} \mathbf{X}_{i}\left(\mathbf{X}^{\top} \hat{\boldsymbol{\Delta}} \mathbf{X}\right)^{-1} \mathbf{X}_{i}^{\top} \hat{\boldsymbol{\Delta}}_{i}^{\frac{1}{2}},
$$

em que $\hat{\boldsymbol{\Delta}}=\operatorname{diag}\left(\hat{\boldsymbol{\Delta}}_{1}, \ldots, \hat{\boldsymbol{\Delta}}_{m}\right)$ e $\boldsymbol{\Delta}_{i}$ definido na equação (2.8).

Já para avaliar a influência das observações no conjunto de valores ajustados da $i$-ésima unidade experimental é recomendado considerar uma medida resumo das quantidades $\hat{h}_{i 11}, \ldots$, $\hat{h}_{i n_{i} n_{i}}$, por exemplo considerando $\hat{h}_{i}=\sum_{j=1}^{n_{i}} \frac{\hat{h}_{i j j}}{n_{i}}$, e considerar o gráfico de índices de $\hat{h}_{i}$. Valores destoantes nos gráficos de índices de $\hat{h}_{i j j}$ e $\hat{h}_{i}$ devem ser avaliados, por exemplo, eliminando as unidades experimentais correspondentes e avaliando se há interferências importantes nos resultados, como por exemplo mudanças inferenciais.

\section{- Pontos Aberrantes}

Uma forma de verificar a qualidade do ajuste de um modelo a um conjunto de dados é através da avaliação de possíveis pontos aberrantes, ou seja, mal ajustados. Como no contexto de EEGs não conhecemos a função de verossimilhança completa dos dados, uma proposta para avaliar a presença de pontos aberrantes é através do resíduo de Pearson padronizado, definido por

$$
r_{p_{i j}}=\frac{\mathbf{e}_{(i j)}^{\top} \hat{\boldsymbol{\Delta}}_{i}^{\frac{1}{2}}\left(\hat{\mathbf{A}}_{i}^{\frac{1}{2}} \hat{\mathbf{W}}_{i}^{\frac{1}{2}}\right)^{-1}\left(\mathbf{y}_{\mathbf{i}}-\hat{\boldsymbol{\mu}}_{i}\right)}{\sqrt{1-\hat{h}_{i j j}}},
$$

em que $\mathbf{e}_{i j}$ é um vetor de tamanho $n_{i}$ com a posição referente à observação $y_{i j}$ contendo o valor 1 e todas as outras posições contendo o valor 0 .

Como em geral não conhecemos a distribuição de $r_{p_{i j}}$, uma proposta é gerar a distribuição empírica (vide, por exemplo, Venezuela, 2003), de $r_{p_{i j}}$ com uma banda de confiança. Programas estão disponíveis para algumas situações particulares, tais como EEGs com marginais Poisson, binomial e gama. Para demais situações é sugerido o gráfico de $r_{p_{i j}}$ contra os valores ajustados de $\hat{y}_{i j}$ ou $\hat{\eta}_{i j}$.

\section{- Pontos Influentes}

A ideia de pontos influentes é detectar no conjunto de dados observações que tenham um efeito desproporcional nas estimativas, em particular nos coeficientes da regressão. Atenção especial deve ser dada àqueles pontos cuja eliminação leva a mudanças inferenciais. O procedimento mais usual consiste em estudar a eliminação de cada observação nas estimativas dos coeficientes da regressão como descrito, no contexto de EEGs, por Venezuela (2003). A distância de Cook (Cook, 1977) é a medida de influência mais utilizada quando se estuda o impacto nas estimativas da eliminação de observações.

A distância de Cook quando se exclui a observação $y_{i j}$ fica aprozimada por

$$
(\mathrm{DC})_{i j}=\left(r_{P_{i} j}\right)^{2} \frac{\hat{h}_{i j j}}{p\left(1-\hat{h}_{i j j}\right)} .
$$

É sugerido o gráfico de índices de $\mathrm{DC}_{i j}$ e avaliar aqueles pontos que destoam em relação aos demais. 


\section{- QIC}

QIC é uma medida de informação proposta por Pan (2001) como uma modificação da medida AIC (Akaike, 1973), que consiste em selecionar o modelo mais parcimonioso dentre os ajustados. Essa medida é útil tanto para a seleção de modelos, quanto para a escolha de uma matriz de correlação.

Venezuela (2007) define duas formas de usar o QIC. A primeira é proposta para a seleção de uma matriz de correlação trabalho, sendo definida por

$$
\mathrm{QIC}(R)=-2 \ell(\hat{\boldsymbol{\beta}}(\mathbf{R}))+2 \operatorname{tr}\left(\hat{\mathbf{S}}_{I} \hat{\mathbf{J}}_{m R}^{-1}\right)
$$

em que $\ell(\cdot)$ denota o logaritmo da função de verossimilhança que gera a função escore equivalente à equação de estimação quando assumimos independência entre as observações, $\hat{\mathbf{S}}_{I}$ denota a matriz de sensibilidade sob a estrutura de independência e $\hat{\mathbf{J}}_{m R}^{-1}$ o estimador robusto sob a estrutura $\mathbf{R}$, os quais são avaliados em $\hat{\boldsymbol{\beta}}(\mathbf{R})$ que é a estimativa de $\boldsymbol{\beta}$ obtida com a matriz trabalho $\mathbf{R}$.

Já para a seleção de variáveis, a fórmula de QIC fica definida por

$$
\mathrm{QIC}_{s}(\mathbf{R})=-2 \ell(\hat{\boldsymbol{\beta}}(\mathbf{R}))+2 p .
$$

Venezuela (2007) alerta que tais medidas só são válidas quando o parâmetro de dispersão $\phi$ é conhecido e único. Quando for desconhecido, a medida QIC é computada utilizando o maior valor estimado para o parâmetro de dispersão. 


\section{Capítulo 3}

\section{Extensão para a Binomial Negativa}

De acordo com Cui e Feng (2008) o modelo binomial negativo original era utilizado para modelar a "propensão à acidentes" por Greenwood e Yule (1920), mas desde então tem sido utilizado em diversas outras aplicações. E hoje em dia é utilizado principalmente para modelar dados de contagem quando o modelo de Poisson não é apropriado dada a sobredispersão.

A regressão binomial negativa é um modelo estatístico incomum, pois apesar de ser referenciado como um único modelo, na verdade existem vários modelos com resposta binomial negativa segundo Hilbe (2011). O que geralmente pode causar muita confusão, já que nem sempre se sabe se o modelo que está sendo empregado tem uma parametrização diferente da que está sendo usada por um outro modelo.

Então é de grande importância definir qual modelo binomial negativo será utilizado nessa dissertação.

A binomial negativa mais tradicional, comumente simbolizada como NB2 (Cameron e Trivedi , 1986), é derivada de uma mistura das ditribuições Poisson e gama. Apesar disso, a origem da binomial negativa não foi uma mistura de Poisson e gama, e sim definida em função da função de probabilidades da binomial. Especificamente ela é caracterizada como o número de fracassos antes da ocorrência do $r$-ésimo sucesso em uma série de ensaios independentes de Bernoulli. Quando $r$ é um valor inteiro, a forma da distribuição é conhecida como Pascal, enquanto que para modelos binomiais negativos o parâmetro $r$ é um número real maior do que zero, apesar de quase nunca ultrapassar 4. O modelo binomial negativo com $r$ igual a zero é Poisson e com $r$ igual a 1 é denominado distribuição geométrica.

É importante entender que o modelo binomial negativo tradicional pode ser modelado usando a função de máxima verossimilhança padrão, ou pode ser modelado como um membro da família de modelos lineares generalizados (MLGs). Sendo que um modelo binomial negativo só é considerado da família exponencial se o seu parâmetro de heterogeneidade entrar no algoritmo como uma constante (Hilbe, 2011).

$\mathrm{Na}$ teoria dos MLGs, a função de ligação é o termo que lineariza a relação entre a média e o preditor linear. Em MLGs, para dados de contagem, o logaritmo da função de verossimilhança pode ser expresso na forma

$$
\ell(\mathbf{y}, \boldsymbol{\theta})=\sum_{i=1}^{n}\left\{y_{i} \theta_{i}-b\left(\theta_{i}\right)+c\left(y_{i}\right)\right\},
$$

em que $\theta_{i}$ é o parâmetro canônico e a média e a variância de $y_{i}$ são expressas, respectivamente, como $b^{\prime}\left(\theta_{i}\right)$ e $b^{\prime \prime}\left(\theta_{i}\right)$.

No modelo binomial negativo canônico a ligação canônica é dada por $\theta=\log \left(\frac{\mu \alpha}{1+\mu \alpha}\right)$, em que $\mu$ é a média e $\alpha$ o parâmetro de heterogeneidade. Já no modelo binomial negativo tradicional é usual utilizar a ligação $\log (\mu)$, que facilita comparações com modelos de Poisson que também usam em geral ligação logarítmica. 


\subsection{Modelos NB-C e NB2}

Vimos que a função de probabilidades da distribuição binomial negativa pode ser definida de diversas formas, mas principalmente de duas maneiras. Hilbe (2011) as define como binomial negativa canônica (NB-C) e como binomial negativa 2 (NB2).

A abordagem tradicional (NB2) sugere uma binomial negativa que surge através da mistura das distribuições Poisson e gama. Vamos supor $y \mid z \sim \operatorname{Poisson}(z)$ e que $z \sim \operatorname{Gama}(\mu, \nu)$ e queremos encontrar a função de probabilidades da variável $y$. Temos então

$$
f(y \mid z)=\frac{e^{-z} z^{y}}{y !} \quad \text { e } \quad g(z ; \mu, \nu)=\frac{1}{\Gamma(\nu)}\left(\frac{z \nu}{\mu}\right)^{\nu} e^{-\frac{\nu}{\mu} z} \frac{1}{z} .
$$

Para encontrarmos a função de probabilidades da variável $y$ devemos obter

$$
f(y ; \mu, \nu)=\int_{0}^{\infty} f(y, z ; \mu, \nu) d z
$$

que pode ser reescrita como

$$
\begin{aligned}
f(y ; \mu, \nu) & =\int_{0}^{\infty} f(y \mid z) g(z ; \mu, \nu) d z \\
& =\int_{0}^{\infty} \frac{1}{y !} \frac{\nu^{\nu}}{\mu^{\nu} \Gamma(\nu)} e^{-z\left(1+\frac{\nu}{\mu}\right)} z^{\nu+y-1} d z \\
& =\frac{1}{y !} \frac{\nu^{\nu}}{\mu^{\nu} \Gamma(\nu)} \int_{0}^{\infty} e^{-z\left(1+\frac{\nu}{\mu}\right)} z^{\nu+y-1} d z .
\end{aligned}
$$

Chamamos $t=z\left(1+\frac{\nu}{\mu}\right)$ e obtemos

$$
\begin{aligned}
f(y ; \mu, \nu) & =\frac{1}{y !} \frac{\nu^{\nu}}{\mu^{\nu} \Gamma(\nu)} \int_{0}^{\infty} e^{-t}\left(\frac{t}{1+\frac{\nu}{\mu}}\right)^{\nu+y-1} \frac{d t}{\left(1+\frac{\nu}{\mu}\right)} \\
& =\frac{1}{y !} \frac{\nu^{\nu}}{\mu^{\nu} \Gamma(\nu)}\left(1+\frac{\nu}{\mu}\right)^{-(\nu+y-1)}\left(1+\frac{\nu}{\mu}\right)^{-1} \int_{0}^{\infty} e^{-t} t^{\nu+y-1} d t .
\end{aligned}
$$

Sabemos que $\Gamma(x)=\int_{0}^{\infty} e^{-t} t^{x-1} d t$, então obtemos

$$
\begin{aligned}
f(y ; \mu, \nu) & =\frac{1}{y !} \frac{\nu^{\nu}}{\mu^{\nu} \Gamma(\nu)}\left(\frac{\mu+\nu}{\mu}\right)^{-(\nu+y)} \Gamma(\nu+y) \\
& =\frac{\Gamma(\nu+y)}{\Gamma(\nu) y !}\left(\frac{\mu}{\mu+\nu}\right)^{\nu+y}\left(\frac{\nu}{\mu}\right)^{\nu} \\
& =\frac{\Gamma(\nu+y)}{\Gamma(\nu) \Gamma(y+1)}\left(\frac{\mu}{\mu+\nu}\right)^{y}\left(\frac{\mu}{\mu+\nu} \frac{\nu}{\mu}\right)^{\nu} \\
f(y ; \mu, \nu) & =\frac{\Gamma(\nu+y)}{\Gamma(\nu) \Gamma(y+1)}\left(\frac{\mu}{\mu+\nu}\right)^{y}\left(\frac{\nu}{\mu+\nu}\right)^{\nu}, y=0,1,2, \ldots
\end{aligned}
$$

Então $y \sim \operatorname{BinomialNegativa~}(\mu, \nu)$ com função de probabilidades dada por

$$
f(y ; \mu, \nu)=\frac{\Gamma(\nu+y)}{\Gamma(\nu) \Gamma(y+1)}\left(\frac{\mu}{\mu+\nu}\right)^{y}\left(\frac{\nu}{\mu+\nu}\right)^{\nu}, y=0,1,2, \ldots
$$

Supondo que $\nu$ é um parâmetro conhecido, podemos reescrever a equação acima como membro da família exponencial

$$
\begin{aligned}
f(y ; \mu, \nu) & =\exp \left\{y \log \left(\frac{\mu}{\mu+\nu}\right)+\nu\left(\frac{\nu}{\mu+\nu}\right)+\log \left(\frac{\Gamma(\nu+y)}{\Gamma(\nu) \Gamma(y+1)}\right)\right\} . \\
& =\exp \left[\nu\left\{\frac{y}{\nu} \log \left(\frac{\mu}{\mu+\nu}\right)+\left(\frac{\nu}{\mu+\nu}\right)\right\}+\log \left(\frac{\Gamma(\nu+y)}{\Gamma(\nu) \Gamma(y+1)}\right)\right] .
\end{aligned}
$$

Podemos ver pela notação da família exponencial (vide, por exemplo, Paula, 2013) que $\phi=\nu$ e 
que $\frac{y}{\nu}$ pertence à família exponencial com

$$
\begin{gathered}
\theta=\log \left(\frac{\mu}{\nu+\mu}\right) \Leftrightarrow \quad e^{\theta}=\frac{\mu}{\nu+\mu}, \\
b(\theta)=-\log \left(\frac{\nu}{\mu+\nu}\right) \Leftrightarrow \quad b(\theta)=-\log \left(1-e^{\theta}\right), \\
b^{\prime}(\theta)=-\frac{\left(-e^{\theta}\right)}{1-e^{\theta}}=\frac{e^{\theta}}{1-e^{\theta}}=\mu^{*}, \\
b^{\prime \prime}(\theta)=\frac{e^{\theta}\left(1-e^{\theta}\right)-e^{\theta}\left(-e^{\theta}\right)}{\left(1-e^{\theta}\right)^{2}}=\frac{e^{\theta}}{\left(1-e^{\theta}\right)^{2}}=\frac{e^{\theta}}{\left(1-e^{\theta}\right)} \frac{1}{\left(1-e^{\theta}\right)}=\mu^{*}\left(1+\mu^{*}\right)=V\left(\mu^{*}\right) \\
\text { e } c(y, \phi)=\log \left(\frac{\Gamma(\nu+y)}{\Gamma(\nu) \Gamma(y+1)}\right) \quad \operatorname{com} \quad \mu^{*}=\mathrm{E}\left(\frac{y}{\nu}\right) .
\end{gathered}
$$

É preciso mencionar que a forma das misturas das variâncias que constituem o núcleo da mistura Poisson-gama é $\mu+\frac{\mu^{2}}{\nu}$, em que $\mu$ é a variância da Poisson e $\frac{\mu^{2}}{\nu}$ é a variância da gama; $\nu$ é o parâmetro de forma da gama e corresponde à dispersão extra do modelo mistura binomial negativo. Dessa maneira há uma relação entre o $\nu$ e o grau de sobredispersão nos dados. Uma binomial negativa se aproxima de uma Poisson quando $\nu$ tende ao infinito. Contudo, o que é feito é a inversão do parâmetro $\nu$ para que se encontre uma relação direta entre o parâmetro e a correlação extra. $\mathrm{O}$ símbolo para o parâmetro de heterogeneidade ou de sobredispersão é $\alpha$. Escrevendo o logaritmo da função de verossimilhança, mas utilizando o parâmetro de heterogeneidade no lugar do $\nu,\left(\nu=\frac{1}{\alpha}\right)$, obtemos

$\ell(\boldsymbol{\mu} ; \mathbf{y}, \alpha)=\sum_{i=1}^{n}\left\{y_{i} \log \left(\frac{\alpha \mu_{i}}{1+\alpha \mu_{i}}\right)-\frac{1}{\alpha} \log \left(1+\alpha \mu_{i}\right)+\log \Gamma\left(y_{i}+\frac{1}{\alpha}\right)-\log \Gamma\left(y_{i}+1\right)-\log \Gamma\left(\frac{1}{\alpha}\right)\right\}$.

A abordagem canônica (NB-C) considera $y$ como sendo o número de fracassos até a ocorrência do $r$-ésimo sucesso, sendo a probabilidade de sucesso denotada por $p$. A função de probabilidades de $y$ fica dada por

$$
f(y ; p, r)=\left(\begin{array}{c}
y+r-1 \\
r-1
\end{array}\right) p^{r}(1-p)^{y}, y=r, r+1, \ldots
$$

No caso da Pascal similarmente à abordagem NB2, quem pertence à família exponencial é $y^{*}=\frac{y}{r}$ e não $y$. Isso porquê a função de variância não pode depender de $r$.

Chamando $y^{*}=\frac{y}{r}$ temos que a função de probabilidades acima pode ser reexpressa na forma

$$
f(y ; p, r)=\left(\begin{array}{c}
r y^{*}+r-1 \\
r-1
\end{array}\right) p^{r}(1-p)^{r y^{*}}, y^{*}=\frac{1}{r}, \frac{2}{r}, \ldots, 1 .
$$

Assim, podemos mostrar que $y^{*}$ pertence à família exponencial

$$
f(y ; p, r)=\exp \left[r\left\{y^{*} \log (1-p)+\log (p)\right\}+\log \left(\begin{array}{c}
r y^{*}+r-1 \\
r-1
\end{array}\right)\right] .
$$

Daí segue que $\phi=r$ e

$$
\begin{gathered}
\theta=\log (1-p) \Leftrightarrow p=1-e^{\theta}, \\
b(\theta)=-\log (p)=-\log \left(1-e^{\theta}\right), \\
b^{\prime}(\theta)=\mu^{*} \Leftrightarrow-\frac{\left(-e^{\theta}\right)}{\left(1-e^{\theta}\right)}=\mu^{*} \Leftrightarrow \frac{(1-p)}{p}=\mu^{*} \quad \mathrm{e}
\end{gathered}
$$




$$
b^{\prime \prime}(\theta)=-\frac{\left[-e^{\theta}\left(1-e^{\theta}\right)-\left(-e^{\theta}\right)\left(-e^{\theta}\right)\right]}{\left(1-e^{\theta}\right)^{2}}=-\frac{-e^{\theta}}{\left(1-e^{\theta}\right)^{2}}=\frac{(1-p)}{p^{2}}=\mu^{*}\left(1+\mu^{*}\right)=V\left(\mu^{*}\right),
$$

em que $\mu^{*}=\mathrm{E}\left(\frac{y}{r}\right)$.

Portanto, podemos reexpressar $p$ em função de $\mu^{*}$, ou seja,

$$
p=\frac{1}{\left(1+\mu^{*}\right)}=\frac{1}{(1+\alpha \mu)},
$$

em que $\alpha=\frac{1}{r}$ e $\mu=\mathrm{E}(y)$. E com essas informações podemos reescrever a função de probabilidades de $y$ na forma

$$
f(y ; \mu, \alpha)=\left(\begin{array}{c}
y+\frac{1}{\alpha}-1 \\
\frac{1}{\alpha}-1
\end{array}\right)\left(\frac{1}{1+\alpha \mu}\right)^{\frac{1}{\alpha}}\left(\frac{\alpha \mu}{1+\alpha \mu}\right)^{y} .
$$

Com isso o logaritmo da função de verossimilhança fica dado por

$\ell(\boldsymbol{\mu} ; \mathbf{y}, \alpha)=\sum_{i=1}^{n}\left\{y_{i} \log \left(\frac{\alpha \mu_{i}}{1+\alpha \mu_{i}}\right)-\frac{1}{\alpha} \log \left(1+\alpha \mu_{i}\right)+\log \Gamma\left(y_{i}+\frac{1}{\alpha}\right)-\log \Gamma\left(y_{i}+1\right)-\log \Gamma\left(\frac{1}{\alpha}\right)\right\}$,

que é expresso na mesma forma da NB2. No entanto, como cada forma utiliza uma função de ligação diferente, quando expressamos o logaritmo da função de verossimilhança em função de $\mathbf{x}_{i}^{\top} \boldsymbol{\beta}$, para $i=1, \ldots, n$, notamos as diferenças entre elas.

Para a NB2, a função de ligação usada é $\log (\boldsymbol{\mu})=\mathbf{x}^{\top} \boldsymbol{\beta}$ ou seja, $\boldsymbol{\mu}=\exp \left(\mathbf{x}^{\top} \boldsymbol{\beta}\right)$. Assim, obtemos

$$
\begin{aligned}
\ell(\boldsymbol{\beta} ; \mathbf{y}, \alpha) & =\sum_{i=1}^{n}\left\{y_{i} \log \left(\frac{\alpha \exp \left(\mathbf{x}_{i}^{\top} \boldsymbol{\beta}\right)}{1+\alpha \exp \left(\mathbf{x}_{i}^{\top} \boldsymbol{\beta}\right)}\right)-\frac{1}{\alpha} \log \left(1+\alpha \exp \left(\mathbf{x}_{i}^{\top} \boldsymbol{\beta}\right)\right)+\log \Gamma\left(y_{i}+\frac{1}{\alpha}\right)\right. \\
& \left.-\log \Gamma\left(y_{i}+1\right)-\log \Gamma\left(\frac{1}{\alpha}\right)\right\} .
\end{aligned}
$$

Já para a NB-C usamos a função de ligação $\log \left(\frac{\alpha \mu}{1+\alpha \mu}\right)=\mathbf{x}^{\top} \boldsymbol{\beta}$, ou seja, $\mu=\frac{1}{\alpha\left[\exp \left(-\mathbf{x}_{i}^{\top} \boldsymbol{\beta}\right)-1\right]}$. Nesse caso, o logaritmo da função de verossimilhança fica dado por

$$
\begin{aligned}
\ell(\boldsymbol{\beta} ; \mathbf{y}, \alpha) & =\sum_{i=1}^{n}\left\{y_{i} \log \left(\frac{\alpha \frac{1}{\alpha\left[\exp \left(-\mathbf{x}_{i}^{\top} \boldsymbol{\beta}\right)-1\right]}}{1+\alpha \frac{1}{\alpha\left[\exp \left(-\mathbf{x}_{i}^{\top} \boldsymbol{\beta}\right)-1\right]}}\right)-\frac{1}{\alpha} \log \left(1+\alpha \frac{1}{\alpha\left[\exp \left(-\mathbf{x}_{i}^{\top} \boldsymbol{\beta}\right)-1\right]}\right)\right. \\
& \left.+\log \Gamma\left(y_{i}+\frac{1}{\alpha}\right)-\log \Gamma\left(y_{i}+1\right)-\log \Gamma\left(\frac{1}{\alpha}\right)\right\} \\
& =\sum_{i=1}^{n}\left\{y_{i}\left(\mathbf{x}_{i}^{\top} \boldsymbol{\beta}\right)+\left(\frac{1}{\alpha}\right) \log \left(1-\exp \left(\mathbf{x}_{i}^{\top} \boldsymbol{\beta}\right)\right)+\log \Gamma\left(y_{i}+\frac{1}{\alpha}\right)-\log \Gamma\left(y_{i}+1\right)-\log \Gamma\left(\frac{1}{\alpha}\right)\right\} .
\end{aligned}
$$

Em geral os softwares estatísticos quando usam a binomial negativa no contexto de EEGs, utilizam o modelo NB2 tradicional. Chamaremos de agora em diante NB2 simplesmente de BN.

\subsection{Ajuste de Modelos com Respostas Indenpendentes Binomial Negativa}

Vamos supor inicialmente que $y_{i j}$ são varáveis aleatórias independentes tais que $y_{i j} \sim \operatorname{BN}\left(\mu_{i j}, \nu\right)$, para $i=1, \ldots, m$ e $j=1, \ldots, n_{i}$. Vamos considerar que $g\left(\mu_{i j}\right)=\eta_{i j}=\mathbf{x}_{i j}^{\top} \boldsymbol{\beta}$, em que $\mathbf{x}_{i j}=$ $\left(x_{i j 1}, \ldots, x_{i j p}\right)^{\top}$ contém valores de variáveis explicativas, $\boldsymbol{\beta}=\left(\beta_{1}, \ldots, \beta_{p}\right)^{\top}, \nu$ é desconhecido e $g(\cdot)$ denota a função de ligação. 
O logaritmo da função de verossimilhança para $\boldsymbol{\theta}=\left(\boldsymbol{\beta}^{\top}, \nu\right)^{\top}$ pode ser expresso na forma

$$
\ell(\boldsymbol{\theta})=\sum_{i=1}^{m} \sum_{j=1}^{n_{i}}\left[\log \left\{\frac{\Gamma\left(\nu+y_{i j}\right)}{\Gamma\left(y_{i j}+1\right) \Gamma(\nu)}\right\}+\nu \log \nu+y_{i j} \log \left(\mu_{i j}\right)-\left(\nu+y_{i j}\right) \log \left(\nu+\mu_{i j}\right)\right],
$$

em que $\mu_{i j}=g^{-1}\left(\eta_{i j}\right)$. Após algumas manipulações algébricas (vide, por exemplo, Paula, 2013, Cap. 4) podemos obter as funções escore para $\boldsymbol{\beta}$ e $\nu$, respectivamente, dadas por

$$
\mathbf{U}_{\beta}=\sum_{i=1}^{n} \mathbf{X}_{i}^{\top} \mathbf{W}_{i} \mathbf{F}_{i}^{-1}\left(\mathbf{y}_{i}-\boldsymbol{\mu}_{i}\right)
$$

em que $\mathbf{X}_{i}$ é uma matriz $n_{i} \times p$ com linhas $\mathbf{x}_{i j}^{\top}, \mathbf{W}_{i}=\operatorname{diag}\left\{w_{i 1}, \ldots, w_{i n_{i}}\right\} \operatorname{com} w_{i j}=\frac{\left(\frac{d \mu_{i j}}{d \eta_{i j}}\right)^{2}}{\mu_{i j}+\nu^{-1} \mu_{i j}^{2}}$, $\mathbf{F}_{i}=\operatorname{diag}\left\{f_{i 1}, \ldots, f_{i n_{i}}\right\} \operatorname{com} f_{i j}=\frac{d \mu_{i j}}{d \eta_{i j}}, \mathbf{y}_{i}=\left(y_{i 1}, \ldots, y_{i n_{i}}\right)^{\top}, \boldsymbol{\mu}_{i}=\left(\mu_{i 1}, \ldots, \mu_{i n_{i}}\right)^{\top} \mathrm{e}$

$$
U_{\nu}=\sum_{i=1}^{m} \sum_{j=1}^{n_{i}}\left[\psi\left(\nu+y_{i j}\right)-\psi(\nu)-\frac{\left(y_{i j}+\nu\right)}{\left(\mu_{i j}+\nu\right)}+\log \left\{\frac{\nu}{\left(\mu_{i j}+\nu\right)}+1\right],\right.
$$

em que $\psi(a)=\frac{\Gamma^{\prime}(a)}{\Gamma(a)}$, para $a>0$, denota a função digama.

Podemos também mostrar que a matriz de informação de Fisher para $\boldsymbol{\beta}$ fica dada por

$$
J_{\beta \beta}=\sum_{i=1}^{m} \mathbf{X}_{i}^{\top} \mathbf{W}_{i} \mathbf{X}_{i}
$$

porém devido à complexidade no cálculo da informação de Fisher para $\nu$ (vide Lawless , 1987), vamos considerar apenas a segunda derivada do logaritmo da função de verossimilhança para $\nu$, dada por

$$
\ddot{L}_{\nu \nu}=\sum_{i=1}^{m} \sum_{j=1}^{n_{i}}\left\{\psi^{\prime}\left(\nu+y_{i j}\right)+\frac{\left(y_{i j}-2 \mu_{i j}-\nu\right)}{\left(\nu+\mu_{i j}\right)^{2}}\right\}+N \nu^{-1}\left\{1-\nu \psi^{\prime}(\nu)\right\},
$$

em que $N=\sum_{i=1}^{m} n_{i}$ e $\psi^{\prime}(\nu)=\frac{d \psi(\nu)}{d \nu}$ denota a função trigama para $\nu>0$. Conforme demonstrado por Lawless (1987) os parâmetros $\boldsymbol{\beta}$ e $\nu$ são ortogonais.

Podemos desenvolver um processo de estimação para obter as estimativas de máxima verossimilhança para $\boldsymbol{\beta}$ e $\nu$ baseado nos métodos escore de Fisher e Newton - Raphson, respectivamente, dados por

$$
\begin{gathered}
\boldsymbol{\beta}^{(h+1)}=\boldsymbol{\beta}^{(h)}+\left(J_{\beta \beta}^{-1}\right)^{(h)} \mathbf{U}_{\beta}^{(h)} \\
\nu^{(h+1)}=\nu^{(h)}-\left(\frac{U_{\nu}^{(h)}}{\ddot{L}_{\nu \nu}^{(h)}}\right),
\end{gathered}
$$

sendo que (3.2) pode ser reescrito na forma

$$
\begin{aligned}
\boldsymbol{\beta}^{(h+1)} & =\boldsymbol{\beta}^{(h)}+\left\{\sum_{i=1}^{m} \mathbf{X}_{i}^{\top} \mathbf{W}_{i}^{(h)} \mathbf{X}_{i}\right\}^{-1} \sum_{i=1}^{m} \mathbf{X}_{i}^{\top} \mathbf{W}_{i}^{(h)}\left(\mathbf{F}_{i}^{-1}\right)^{(h)}\left(\mathbf{y}_{i}-\boldsymbol{\mu}_{i}^{(h)}\right) \\
& =\left\{\sum_{i=1}^{m} \mathbf{X}_{i}^{\top} \mathbf{W}_{i}^{(h)} \mathbf{X}_{i}\right\}^{-1}\left\{\sum_{i=1}^{m} \mathbf{X}_{i}^{\top} \mathbf{W}_{i}^{(h)} \mathbf{y}_{i}^{*(h)}\right\},
\end{aligned}
$$

para $h=0,1, \ldots$, em que $\mathbf{y}_{i}^{*}=\mathbf{X}_{i} \boldsymbol{\beta}-\mathbf{F}_{i}^{-1}\left(\mathbf{y}_{i}-\boldsymbol{\mu}_{i}\right)$ para $i=1, \ldots, m$. O processo iterativo (3.2 3.3) deve ser inicializado em $\boldsymbol{\beta}^{(0)}$ e $\nu^{(0)}$. 
Sob certas condições de regularidade e para $m$ grande temos que

$$
\hat{\boldsymbol{\beta}} \sim \mathrm{N}_{p}\left(\boldsymbol{\beta}, \mathbf{J}_{\beta \beta}^{-1}\right) \quad \text { e } \quad \hat{\nu} \sim \mathrm{N}\left(\nu, J_{\nu \nu}^{-1}\right),
$$

em que $J_{\nu \nu}=\mathrm{E}\left(-\ddot{L}_{\nu \nu}\right)$, além disso devido à ortogonalidade entre $\boldsymbol{\beta}$ e $\nu$, segue assintoticamente que $\operatorname{Cov}(\hat{\boldsymbol{\beta}}, \hat{\nu})=\mathbf{0}$.

Estimativas consistentes para $\operatorname{Var}(\hat{\boldsymbol{\beta}})$ e $\operatorname{Var}(\hat{\nu})$ ficam, respectivamente, dadas por

$$
\hat{\mathbf{J}}_{\beta \beta}^{-1}=\left\{\sum_{i=1}^{m} \mathbf{X}_{i}^{\top} \hat{\mathbf{W}}_{i} \mathbf{X}_{i}\right\}^{-1} \quad \text { e } \quad \hat{J}_{\nu \nu}^{-1}=-\hat{\ddot{L}}_{\nu \nu}^{-1} .
$$

\subsubsection{Métodos de Diagnóstico}

Note que na convergência do processo iterativo dado em (3.5) temos que

$$
\begin{aligned}
\hat{\boldsymbol{\beta}} & =\left\{\sum_{i=1}^{m} \mathbf{X}_{i}^{\top} \hat{\mathbf{W}}_{i} \mathbf{X}_{i}\right\}^{-1}\left\{\sum_{i=1}^{m} \mathbf{X}_{i}^{\top} \hat{\mathbf{W}}_{i} \hat{\mathbf{y}}_{i}^{*}\right\} \\
& =\left(\mathbf{X}^{\top} \hat{\mathbf{W}} \mathbf{X}\right)^{-1}\left(\mathbf{X}^{\top} \hat{\mathbf{W}}^{*}\right),
\end{aligned}
$$

em que $\mathbf{X}=\left[\mathbf{X}_{1}^{\top}, \ldots, \mathbf{X}_{m}^{\top}\right]^{\top}, \mathbf{W}=\operatorname{diag}\left\{\mathbf{W}_{1}, \ldots, \mathbf{W}_{m}\right\}$ e $\mathbf{y}=\left[\mathbf{y}_{1}^{\top}, \ldots, \mathbf{y}_{m}^{\top}\right]^{\top}$.

Portanto, $\hat{\boldsymbol{\beta}}$ pode ser interpretado como sendo a solução de mínimos quadrados da regressão linear de $\hat{\mathbf{W}}^{\frac{1}{2}} \hat{\mathbf{y}}$ nas colunas da matriz $\hat{\mathbf{W}}^{\frac{1}{2}} \mathbf{X}$. Os valores preditos dessa regressão ficam dados por

$$
\begin{aligned}
\hat{\mathbf{W}}^{\frac{1}{2}} \mathbf{X} \hat{\boldsymbol{\beta}} & =\left\{\hat{\mathbf{W}}^{\frac{1}{2}} \mathbf{X}\left(\mathbf{X}^{\top} \hat{\mathbf{W}} \mathbf{X}\right)^{-1} \mathbf{X}^{\top} \hat{\mathbf{W}}^{\frac{1}{2}}\right\} \hat{\mathbf{W}}^{\frac{1}{2}} \hat{\mathbf{y}} \\
& =\hat{\mathbf{H}} \hat{\mathbf{W}}^{\frac{1}{2}} \hat{\mathbf{y}}
\end{aligned}
$$

em que $\hat{\mathbf{H}}=\hat{\mathbf{W}}^{\frac{1}{2}} \mathbf{X}\left(\mathbf{X}^{\top} \hat{\mathbf{W}} \mathbf{X}\right)^{-1} \mathbf{X}^{\top} \hat{\mathbf{W}}^{\frac{1}{2}}$ funciona como uma matriz de projeção desta regressão. Essa matriz pode ser expressa da forma

$$
\hat{\mathbf{H}}=\operatorname{diag}\left\{\hat{\mathbf{H}}_{1}, \ldots, \hat{\mathbf{H}}_{m}\right\},
$$

em que $\mathbf{H}_{i}=\mathbf{W}_{i}^{\frac{1}{2}} \mathbf{X}_{i}\left(\mathbf{X}^{\top} \mathbf{W} \mathbf{X}\right)^{-1} \mathbf{X}_{i}^{\top} \mathbf{W}_{i}^{\frac{1}{2}}$, para $i=1, \ldots, m$.

Como é um caso de réplicas estamos interessados nas observações individualmente, assim uma medida de alavanca para a $j$-ésima observação da $i$-ésima réplica fica dada por $\hat{h}_{i j j}$, que corresponde ao $j$-ésimo elemento da diagonal principal da matriz $\hat{\mathbf{H}}_{i}$. Podemos escrever

$$
h_{i j j}=\frac{\left(\frac{d \mu_{i j}}{d \eta_{i j}}\right)^{2}}{\left\{\mu_{i j}^{2} \nu^{-1}+\mu_{i j}\right\}^{\top}} \mathbf{x}_{i j}^{\top}\left(\mathbf{X}^{\top} \mathbf{W} \mathbf{X}\right)^{-1} \mathbf{x}_{i j},
$$

para $i=1, \ldots, m$ e $j=1, \ldots, n_{i}$. O gráfico de índices de $\hat{h}_{i j j}$ pode revelar pontos de alavanca na regressão de mínimos quadrados acima.

Em particular, para a ligação logarítmica em que $\log \left(\mu_{i j}\right)=\eta_{i j}$, temos que $\frac{d \mu_{i j}}{d \eta_{i j}}=\mu_{i j}$, e portanto

$$
h_{i j j}=\frac{\nu \mu_{i j}}{\left(\mu_{i j}+\nu\right)} \mathbf{x}_{i j}^{\top}\left(\mathbf{X}^{\top} \mathbf{W} \mathbf{X}\right)^{-1} \mathbf{x}_{i j}
$$

Com relação aos resíduos, como conhecemos a verossimilhança completa, podemos fazer uma adaptação do resíduo componente do desvio apresentado, por exemplo, em Paula (2013, Cap. 4) para o caso do binomial negativo com réplicas. Esse resíduo fica dado por 


$$
t_{D_{i j}}=\frac{d\left(y_{i j} ; \hat{\mu}_{i j}\right)}{\sqrt{1-\hat{h}_{i j j}}}
$$

em que $d^{2}\left(y_{i j} ; \hat{\mu}_{i j}\right)$ denota o $(i, j)$-ésimo elemento da funçaõ desvio $D(y ; \hat{\mu})=\sum_{i=1}^{m} \sum_{j=1}^{n_{i}} d^{2}\left(y_{i j} ; \hat{\mu}_{i j}\right)$, sendo que

$$
d\left(y_{i j} ; \hat{\mu}_{i j}\right)= \begin{cases} \pm \sqrt{2}\left[\log \left\{\frac{\hat{\mu}_{i j}+\nu}{y_{i j}+\nu}\right\}+y_{i j} \log \left\{\frac{y_{i j}\left(\hat{\mu}_{i j}+\nu\right)}{\hat{\mu}_{i j}\left(y_{i j}+\nu\right)}\right\}\right]^{\frac{1}{2}} & \text { para } y_{i j}>0 \\ \pm \sqrt{2}\left[\nu \log \left\{\frac{\hat{\mu}_{i j}+\nu}{\nu}\right\}\right] & \text { para } y_{i j}=0 .\end{cases}
$$

O gráfico normal de probabilidades para $t_{D_{i j}}$ pode revelar eventuais afastamentos de $y_{i j}$ da distribuição binomial negativa, bem como a presença de observações aberrantes.

Outra possibilidade é aplicar o resíduo quantílico (Dunn e Smyth, 1996) definido por

$$
r_{q_{i}}=\Phi^{-1}\left\{F\left(y_{i j} ; \hat{\mu}_{i j}, \hat{\nu}\right)\right\},
$$

em que $F(y ; \mu, \nu)$ denota a função de distribuição acumulada da $B N(\mu, \nu)$ e $\Phi^{-1}(\cdot)$ a função de distribuição acumulada da $N(0,1)$. Se $y_{i j}$ segue uma $B N\left(\mu_{i j}, \nu\right)$ então $r_{q_{i}}$ pode ser aproximado para amostras grandes por uma $N(0,1)$.

Finalmente, a distância de Cook fica dada por

$$
(D C)_{i j}=\frac{\hat{h}_{i j j}}{\left(1-\hat{h}_{i j j}\right)} \hat{r}_{p_{i j}}^{2}
$$

em que $r_{p_{i j}}=\frac{\left(y_{i j}-\mu_{i j}\right)}{\left(\mu_{i j}^{2} \nu^{-1}+\mu_{i j}\right)}$ denota o resíduo de Pearson. O gráfico de índices de $(D C)_{i j}$ pode revelar a presença de observações individualmente influentes nas estimativas dos coeficientes estimados da regressão.

\subsection{Ajuste de Modelos com Respostas Correlacionadas Binomial Negativa}

Vamos fazer agora uma extensão dos resultados apresentados no Capítulo 2 para o caso em que as distribuições marginais das respostas são binomial negativa. Como foi visto na Seção 3.1 a binomial negativa pertence à família exponencial quando $\nu$ é conhecido. Contudo, vamos inicialmente desenvolver as EEGs supondo $\nu$ desconhecido.

Considere, portanto, o seguinte modelo:

(i) $\mathbf{y}_{i}=\left(y_{i 1}, \ldots, y_{i n_{i}}\right)^{\top}$ tal que $y_{i j} \sim \mathrm{BN}\left(\mu_{i j}, \nu\right)$,

(ii) $g\left(\mu_{i j}\right)=\eta_{i j}=\mathbf{x}_{i j}^{\top} \boldsymbol{\beta}$,

(iii) $\operatorname{Corr}\left(\mathbf{y}_{i}\right)=\mathbf{R}_{i}(\boldsymbol{\rho})$,

em que $\mathbf{x}_{i j}=\left(x_{i j 1}, \ldots, x_{i j p}\right)^{\top}$ contém valores de variáveis explicativas, $\boldsymbol{\beta}=\left(\beta_{1}, \ldots, \beta_{p}\right)^{\top}, \mathbf{R}_{i}(\boldsymbol{\rho})$ denota a matriz trabalho com $\boldsymbol{\rho}=\left(\rho_{1}, \ldots, \rho_{s}\right)^{\top}$.

Similarmente à família exponencial vamos denotar $\mathbf{u}_{i}=\mathbf{y}_{i}-\boldsymbol{\mu}_{i}$, em que $\mathbf{u}_{i}=\left(u_{i 1}, \ldots, u_{i n_{i}}\right)^{\top}$ com $u_{i j}=y_{i j}-\mu_{i j}$, para $i=1, \ldots, m$ e $j=1, \ldots, n_{i}$. Daí segue

$$
\frac{\partial \mathbf{u}_{i}}{\partial \boldsymbol{\beta}^{\top}}=-\frac{\partial \boldsymbol{\mu}_{i}}{\partial \boldsymbol{\beta}^{\top}}=\frac{\partial \boldsymbol{\mu}_{i}}{\partial \boldsymbol{\eta}_{i}} \frac{\partial \boldsymbol{\eta}_{i}}{\partial \boldsymbol{\beta}^{\top}}=-\mathbf{F}_{i} \mathbf{X}_{i}=-\mathbf{D}_{i},
$$

e portanto

$$
\mathrm{E}\left(\frac{\partial \mathbf{u}_{i}}{\partial \boldsymbol{\beta}^{\top}}\right)^{\top}=-\mathbf{X}_{i}^{\top} \mathbf{F}_{i}=-\mathbf{D}_{i}^{\top}
$$


Além disso, obtemos

$$
\operatorname{Var}\left(\mathbf{u}_{i}\right)=\operatorname{Var}\left(\mathbf{y}_{i}\right)=\mathbf{A}_{i}^{\frac{1}{2}} \mathbf{R}_{i}(\boldsymbol{\rho}) \mathbf{A}_{i}^{\frac{1}{2}}=\boldsymbol{\Omega}_{i},
$$

em que $\mathbf{A}_{i}=\operatorname{diag}\left\{\mu_{i j}^{2} \nu^{-1}+\mu_{i j}\right\}$.

As EEGs para $\boldsymbol{\beta}$ ficam dadas por

$$
\boldsymbol{\Psi}_{m G}\left(\hat{\boldsymbol{\beta}}_{G}\right)=0,
$$

em que $\boldsymbol{\Psi}_{m G}(\boldsymbol{\beta})=\sum_{i=1}^{m} \mathbf{D}_{i}^{\top} \boldsymbol{\Omega}_{i}^{-1}\left(\mathbf{y}_{i}-\boldsymbol{\mu}_{i}\right)=\sum_{i=1}^{m} \mathbf{X}_{i}^{\top} \mathbf{F}_{i} \boldsymbol{\Omega}_{i}^{-1}\left(\mathbf{y}_{i}-\boldsymbol{\mu}_{i}\right)$. Um processo iterativo (supondo $\boldsymbol{\rho}$ e $\nu$ fixados) para obter $\hat{\boldsymbol{\beta}}_{G}$ fica dado por

$$
\boldsymbol{\beta}_{G}^{(h+1)}=\boldsymbol{\beta}_{G}^{(h)}+\left\{\sum_{i=1}^{m} \mathbf{D}_{i}^{(h) \top} \boldsymbol{\Omega}_{i}^{-1(h)} \mathbf{D}_{i}^{(h)}\right\}^{-1} \sum_{i=1}^{m} \mathbf{D}_{i}^{(h) \top} \boldsymbol{\Omega}_{i}^{-1(h)}\left(\mathbf{y}_{i}-\boldsymbol{\mu}_{i}^{(h)}\right),
$$

para $h=0,1, \ldots$. O processo iterativo (3.8) deve ser alternado com processos iterativos para obter $\hat{\nu}$ e $\hat{\boldsymbol{\rho}}$. No caso de $\hat{\boldsymbol{\rho}}$ devemos alternar (3.8) com as estimativas consistentes para $\boldsymbol{\rho}$ dependendo da estrutura de correlação assumida para $\mathbf{R}_{i}(\boldsymbol{\rho})$. Com relação a $\nu$ sugerimos a utilização do processo iterativo (3.3) assumido para o caso de respostas independentes. Estudos de simulação deveriam ser desenvolvidos para avaliar a consistência das estimativas $\left(\hat{\boldsymbol{\beta}}_{G}^{\top}, \hat{\nu}, \hat{\boldsymbol{\rho}}\right)^{\top}$. Contudo, devido às dificuldades computacionais em gerar variáveis aleatórias $\mathbf{y}_{i}=\left(y_{i 1}, \ldots, y_{i n_{i}}\right)^{\top}$ com marginais binomial negativa e estrutura de correlação $\mathbf{R}_{i}(\boldsymbol{\rho})$ esses estudos não foram desenvolvidos.

Seguindo notação do Capítulo 2, o processo iterativo dado em (3.8) para obter $\hat{\boldsymbol{\beta}}_{G}$ pode ser reexpresso como um processo iterativo de mínimos quadrados reponderados

$$
\boldsymbol{\beta}_{G}^{(h+1)}=\left\{\sum_{i=1}^{m} \mathbf{X}_{i}^{\top} \boldsymbol{\Delta}_{i}^{(h)} \mathbf{X}_{i}\right\}^{-1} \sum_{i=1}^{m} \mathbf{X}_{i}^{\top} \boldsymbol{\Delta}_{i}^{(h)} \mathbf{y}_{i}^{*(h)},
$$

para $h=0,1, \ldots$, em que $\boldsymbol{\Delta}_{i}=\mathbf{W}_{i}^{\frac{1}{2}} \mathbf{R}_{i}^{-1}(\boldsymbol{\rho}) \mathbf{W}_{i}^{\frac{1}{2}}$ e $\mathbf{y}_{i}^{*}=\mathbf{X}_{i}^{\top} \boldsymbol{\beta}+\mathbf{F}_{i}^{-1}\left(\mathbf{y}_{i}-\boldsymbol{\mu}_{i}\right)$, com $\mathbf{W}_{i}=$ $\operatorname{diag}\left\{w_{i 1}, \ldots, w_{i n_{i}}\right\}$ e $w_{i j}=\frac{\left(\frac{d \mu_{i j}}{d \eta_{j}}\right)^{2}}{\left\{\nu^{-1} \mu_{i j}^{2}+\mu_{i j}\right\}}$, para $i=1, \ldots, m$ e $j=1, \ldots, n_{i}$.

Novamente, usando resultados do Capítulo 2 temos que a matriz de variância-covariância assintótica para $\hat{\boldsymbol{\beta}}_{G}$ fica estimada por

$$
\widehat{\operatorname{Var}}\left(\hat{\boldsymbol{\beta}}_{G}\right)=\hat{\mathbf{J}}_{m G}^{-1}
$$

em que

$$
\mathbf{J}_{m G}^{-1}=\left\{\sum_{i=1}^{m} \mathbf{S}_{i}\right\}^{-1}\left\{\sum_{i=1}^{m} \mathbf{D}_{i}^{\top} \boldsymbol{\Omega}_{i}^{-1} \mathbf{u}_{i} \mathbf{u}_{i}^{\top} \boldsymbol{\Omega}_{i}^{-1} \mathbf{D}_{i}\right\}\left\{\sum_{i=1}^{m} \mathbf{S}_{i}\right\}^{-1},
$$

$\operatorname{com} \mathbf{S}_{i}=-\mathbf{X}_{i}^{\top} \mathbf{F}_{i} \boldsymbol{\Omega}_{i}^{-1} \mathbf{F}_{i} \mathbf{X}_{i}, \boldsymbol{\Omega}=\mathbf{A}_{i}^{\frac{1}{2}} \mathbf{R}_{i}(\boldsymbol{\rho}) \mathbf{A}_{i}^{\frac{1}{2}}, \mathbf{u}_{i}=\mathbf{y}_{i}-\boldsymbol{\mu}_{i}$ e $\mathbf{A}_{i}=\operatorname{diag}\left\{\mu_{i j}^{2} \nu^{-1}+\mu_{i j}\right\}$. O estimador $\hat{\mathbf{J}}_{m G}^{-1}$ deve ser avaliado em $\left(\hat{\boldsymbol{\beta}}_{G}^{\top}, \hat{\nu}, \hat{\boldsymbol{\rho}}\right)^{\top}$.

Se $\boldsymbol{\rho}$ e $\nu$ são estimados de forma consistente temos que para $m$ grande $\hat{\boldsymbol{\beta}}_{G} \sim \mathrm{N}_{p}\left(\boldsymbol{\beta}, \mathbf{J}_{m G}^{-1}\right)$.

Alternativamente, podemos supor que $\nu$ é conhecido. Contudo, precisaremos de algum critério adicional para escolher uma estimativa para $\nu$. Uma sugestão é considerar um conjunto de valores a partir da estimativa de máxima verossimilhança para $\nu$ supondo respostas independentes, e a partir daí selecionar um valor para $\nu$ minimizando a medida de informação

$$
\mathrm{QIC}_{s}(\mathbf{R})=-2 \ell(\hat{\boldsymbol{\beta}}(\mathbf{R}))+2 p,
$$

em que $\ell(\boldsymbol{\beta})$ denota o logaritmo da funçaõ de verossimilhança para $\boldsymbol{\beta}$ supondo respostas independentes e $\nu$ fixado, enquanto $\hat{\boldsymbol{\beta}}(\mathbf{R})$ denota a estimativa $\hat{\boldsymbol{\beta}}_{G}$ obtida supondo a estrutura $\mathbf{R}(\hat{\boldsymbol{\rho}}) \operatorname{com} \hat{\boldsymbol{\rho}}$ sendo a estimativa consistente para $\boldsymbol{\rho}$. 


\subsubsection{Métodos de Diagnóstico}

A principal diferença das metodologias de diagnóstico a serem apresentadas neste seção com relação aos procedimentos apresentados na Seção 3.2.1 é que não conhecemos mais a verossimilhança completa, apenas as distribuições marginais das respostas. Assim, faremos uma adaptação dos procedimentos de diagnóstico apresentados no Capítulo 2 para o modelo binomial negativo com respostas correlacionadas.

O gráfico de índices de $\hat{h}_{i j j}$ pode revelar pontos de alavanca como foi definido em (2.11). Temos que

$$
\hat{\mathbf{H}}_{i}=\hat{\boldsymbol{\Delta}}_{i}^{\frac{1}{2}} \mathbf{X}_{i}\left(\mathbf{X}^{\top} \hat{\boldsymbol{\Delta}} \mathbf{X}\right)^{-1} \mathbf{X}_{i}^{\top} \hat{\boldsymbol{\Delta}}_{i}^{\frac{1}{2}}
$$

em que $\hat{\boldsymbol{\Delta}}=\operatorname{diag}\left(\hat{\boldsymbol{\Delta}}_{1}, \ldots, \hat{\boldsymbol{\Delta}}_{m}\right)$ e $\boldsymbol{\Delta}_{i}=\mathbf{W}_{i}^{\frac{1}{2}} \mathbf{R}_{i}^{-1}(\boldsymbol{\rho}) \mathbf{W}_{i}^{\frac{1}{2}}$. Logo, o $j$-ésimo elemento da diagonal principal da matriz $\hat{\mathbf{H}}_{i}$ fica dado por

$$
h_{i j j}=\delta_{i j} \mathbf{x}_{i j}^{\top}\left(\mathbf{X}^{\top} \boldsymbol{\Delta} \mathbf{X}\right)^{-1} \mathbf{x}_{i j}
$$

em que $\delta_{i j}$ é um elemento da matriz $\boldsymbol{\Delta}_{i}$.

Com relação à resíduos, como não conhecemos a verossimilhança completa utilizaremos o resíduo de Pearson padronizado que foi definido em (2.12) e que fica dado por

$$
r_{p_{i j}}=\frac{\mathbf{e}_{(i j)}^{\top} \hat{\boldsymbol{\Delta}}_{i}^{\frac{1}{2}}\left(\hat{\mathbf{A}}_{i}^{\frac{1}{2}} \hat{\mathbf{W}}_{i}^{\frac{1}{2}}\right)^{-1}\left(\mathbf{y}_{\mathbf{i}}-\hat{\boldsymbol{\mu}}_{i}\right)}{\sqrt{1-\hat{h}_{i j j}}}=\frac{\mathbf{e}_{(i j)}^{\top} \hat{\boldsymbol{\Delta}}_{i}^{\frac{1}{2}}\left(\hat{\mathbf{F}}_{i}\right)^{-1}\left(\mathbf{y}_{\mathbf{i}}-\hat{\boldsymbol{\mu}}_{i}\right)}{\sqrt{1-\hat{h}_{i j j}}},
$$

em que $\mathbf{e}_{i j}$ é um vetor de tamanho $n_{i}$ com a posição referente à observação $y_{i j}, \mathbf{F}_{i}=\operatorname{diag}\left\{f_{i 1}, \ldots, f_{i n_{i}}\right\}$ $\operatorname{com} f_{i j}=\frac{d \mu_{i j}}{d \eta_{i j}}$, que em particular para a ligação logarítmica fica $f_{i j}=\mu_{i j}$. Para detectar pontos aberrantes podemos analisar o gráfico de $r_{S D_{i j}}$ contra os valores ajustados $\hat{y}_{i j}$ ou $\hat{\eta}_{i j}$.

Finalmente, temos a distância de Cook conforme dado em (2.13), definida por

$$
(\mathrm{DC})_{i j}=\left(r_{P_{i} j}\right)^{2} \frac{\hat{h}_{i j j}}{p\left(1-\hat{h}_{i j j}\right)} .
$$

O gráfico de índices de $(\mathrm{DC})_{i j}$ pode revelar a presença de pontos influentes.

\subsection{Aspectos Computacionais}

O ajuste de modelos binomial negativo está presente nos softwares de estatística mais conhecidos (SAS, Stata e R), principalmente quando as respostas são independentes. Contudo, como o modelo binomial negativo com dois parâmetros desconhecidos não é considerado da família exponencial, o que os softwares estatísticos fazem quando queremos ajustar um modelo binomial negativo como um MLG é a estimação do parâmetro de dispersão numa subrotina fora do algoritmo MLG, e colocando esse valor como constante no algoritmo MLG. Também está disponível nos softwares a opção do usuário especificar um valor para esse parâmetro.

Quando queremos estimar os parâmetros do modelo binomial negativo usando o algoritmo de máxima verossimilhança, tanto $\mu$ quanto $\alpha$ são estimados. Para as funções do Stata glm, do SAS Genmod e do R glm.nb (do pacote MASS), assim como em outros softwares que são mencionados em Hilbe (2014), o parâmetro de dispersão é calculado por máxima verossimilhança e depois colocado no algoritmo MLG. A função do R nb.glm faz o mesmo, mas não utiliza o parâmetro de dispersão, e sim o inverso dele $\left(\nu=\frac{1}{\alpha}\right)$. Há porém uma função no R que faz a estimação usando o parâmetro de dispersão $\alpha$ que é a nbinomial do pacote CoUnT.

Quando colocamos a suposição de que existe uma dependência entre as observações, o modelo EEG BN, segue de certa forma as mesmas ideias de quando se considerava uma independência entre as observações. Ou seja, a maior parte dos softwares, considera o modelo binomial negativo 
como da família exponencial. Essas funções permitem que o parâmetro de heterogeneidade, $\alpha$, seja estimado em uma subrotina fora do MLG, com o valor resultante entrando de volta no algoritmo MLG como uma constante.

Esse método não está, porém, disponível no comando primário de EEGs no Stata, xtgee, nem no GENMOD do SAS, que com a opção REPEATED prevê um modelo de EEGs. O algoritmo do SAS emprega um valor de $\alpha$ para todas as estruturas baseado no valor obtido usando um modelo padrão ou independente. E até agora as funções do R gee, geeglm e yags, todas que fornecem capacidade de modelar EEGs, falham em acomodar a família binomial negativa.

Quando ajustamos modelos EEG BN usando o Stata, primeiro é necessário modelar a máximaverossimilhança de uma binomial negativa usando nbreg ou glm, fam(nb $\mathbf{m l})$, e então inserir o valor estimado de $\alpha$ na opção de família de xtgee, isto é, $\operatorname{fam}(n b \alpha)$.

Hilbe (2011) explica que quando ajustamos modelos EEG BN, o comando xtgee do Stata funciona de uma maneira contraintuitiva. $\mathrm{O}$ valor de $\alpha$, quando entra na opção da família como constante, é invertido pelo algoritmo, produzindo um modelo com um $\alpha$ mostrado de $\frac{1}{\alpha}$. O logaritmo da função de verossimilhança também é parametrizado com um $\alpha$ invertido. Relembre da discussão da parametrização da função de probabilidades da NB2 $\operatorname{com} \alpha=\frac{1}{\nu}$. Em qualquer caso, a inversão em efeito cancela a segunda inversão, mantendo a função de probabilidades que é mostrada na equação (3.1). A parte confusa disso tudo resta com o $\alpha$ que é mostrado na saída das EEGs. Infelizmente não há nenhuma documentação nos manuais do Stata ou arquivos do help em relação a essa inversão.

Como em qualquer análise estatística, a seleção do modelo apropriado é um problema importante apesar de ser pouco estudado para EEGs por um longo tempo(Pan, 2001). Um dos métodos de diagnóstico para EEGs visto na Seção 2.4 que já está presente nos softwares estatísticos é o QIC. Cui et al. (2007) desenvolveram um programa computacional para implementar o método QIC para uma variedade de estruturas de correlação, funções de ligação, e distribuições estatísticas que estão disponíveis no software Stata. As distribuições estatísticas incluem Bernoulli, normal, Poisson, gama, Gaussiana inversa, e binomial negativa. Em Cui et al. (2007) é apresentado um caso especial da binomial negativa em que o parâmetro de dispersão $\alpha=1$, e em Cui e Feng (2008) é apresentado um modelo binomial negativo geral, ou seja, em que $\alpha$ possa ser qualquer valor fixado.

$\mathrm{O}$ valor do QIC pode ser usado para selecionar a melhor estrutura de correlação e o melhor modelo na análise através de EEGs. Usualmente, a melhor estrutura de correlação é selecionada baseada no modelo completo com todas as variáveis explicativas primeiro (Hardin e Hilbe, 2003). A estrutura de correlação com menor valor de QIC é escolhida como melhor estrutura de correlação. Então, sob a melhor estrutura de correlação o modelo de médias com menor valor QIC é escolhido como o melhor modelo através de EEGs.

Além disso, existem também rotinas desenvolvidas e encontradas no site http://www.ime.usp. $\mathrm{br} /$ giapaula/cursospos.htm, que fazem os gráficos das medidas de diagnóstico mostradas na Seção 2.4 no software R. Essas rotinas estão disponíveis tanto para MLGs quanto para EEGs.

\subsection{Método Alternativo}

Outra forma encontrada para trabalhar com modelos com respostas binomial negativas dependentes é através de cópulas Gaussianas, como foi proposto na dissertação de Trindade (2014). A análise de verossimilhança para modelos marginais não normais não é muito conhecida, pois há uma grande dificuldade na identificação das distribuições multivariadas para respostas discretas, apesar das várias vantagens teóricas e práticas. Essa alternativa é utilizada principalmente por não haver uma função no software $\mathrm{R}$ que resolva EEGs para a resposta binomial negativa.

Segundo Trindade (2014) uma cópula é definida como uma distribuição de probabilidade multivariada que tem distribuições marginais de cada variável conhecidas, e essas são usadas para descrever a dependência entre elas. Os modelos de regressão que usam cópulas Gaussinas são modelos flexíveis, mas com uma estrutura de análise para dados dependentes. As análises de verossimilhança dos modelos de regressão marginais para respostas correlacionadas não normais baseadas em cópulas Gaussinas foram desenvolvidas por Masarotto et al. (2012). 
Para este trabalho vamos entender um pouco da teoria usada nesse método. Seja $\mathbf{y}=\left(y_{1}, \ldots, y_{m}\right)^{\top}$ um vetor com $m$ variáveis aleatórias correlacionadas contínuas ou discretas e considere que o objetivo é avaliar como a distribuição de $y_{i}$ varia de acordo com o vetor $p$-dimensional $\mathbf{x}_{i}=\left(x_{i 1}, \ldots, x_{i p}\right)^{\top}$. Considere $p_{i}\left(y_{i} ; \boldsymbol{\lambda}\right)=p\left(y_{i} \mid \mathbf{x}_{i} ; \boldsymbol{\lambda}\right)$ a função densidade de probabilidades de $y_{i}$ dado $\mathbf{x}_{i}$. Sendo assim o que identifica o modelo de regressão é $p_{i}\left(y_{i} ; \boldsymbol{\lambda}\right)$. Podemos expressar o modelo de regressão como

$$
y_{i}=g\left(\mathbf{x}_{i}, \epsilon_{i} ; \boldsymbol{\lambda}\right), i=1, \ldots m,
$$

em que $g(\cdot)$ é uma função adequada dos regressores $\mathbf{x}_{i}$ e de uma variável estocástica $\epsilon_{i}$, comumente chamada de componente de erro, e $\boldsymbol{\lambda}$ é um vetor de parâmetros desconhecidos. Entre as várias especificações para $g(\cdot)$, a que é sugerida como mais útil por Masarotto et al. (2012) é a função quantil

$$
y_{i}=F_{i}^{-1}\left(\Phi\left(\epsilon_{i}\right) ; \boldsymbol{\lambda}\right), i=1, \ldots, m,
$$

em que $\epsilon_{i}$ é uma variável normal padrão e $F_{i}(\cdot ; \boldsymbol{\lambda})=F\left(\cdot \mid \mathbf{x}_{i} ; \boldsymbol{\lambda}\right)$ a função distribuição de $y_{i}$ dados $\mathbf{x}_{i}$ e $\Phi(\cdot)$ a função de distribuição normal padrão. Pelo teorema da transformação da integral, o modelo (3.9) garante a distribuição marginal desejada para a resposta $y_{i}$ e especifica $\epsilon_{i}$ nos termos de um erro normal.

Para o modelo log-linear de Poisson temos o seguinte:

$$
F_{i}\left(y_{i} ; \boldsymbol{\lambda}\right)=\sum_{k=0}^{y_{i}} \frac{e^{-\mu_{i}} \mu_{i}^{k}}{k !}
$$

em que $\mu_{i}=\exp \left(\mathbf{x}_{i}^{\top} \boldsymbol{\beta}\right), \boldsymbol{\lambda}=\boldsymbol{\beta}$. A especificação do modelo fica completa assumindo que o vetor de erros $\boldsymbol{\epsilon}=\left(\epsilon_{1}, \ldots, \epsilon_{m}\right)^{\top}$ segue distribuição normal multivariada,

$$
\epsilon \sim \mathrm{N}_{p}(0, \Omega)
$$

em que $\boldsymbol{\Omega}$ é uma matriz de correlação (Masarotto et al., 2012; Song, 2000). De forma eficiente a especificação do modelo separa o componente marginal (3.9) do componente dependente (3.10), sendo esse descrito em função de um processo normal multivariado. Uma padronização da matriz de correlação $\boldsymbol{\Omega}$ como uma função de um vetor de parâmetros $\boldsymbol{\varsigma}$ permite que várias formas de dependência sejam modeladas. $\mathrm{O}$ vetor de parâmetros dependentes $(\boldsymbol{\varsigma})$ juntamente com o vetor de parâmetros do modelo original $(\boldsymbol{\lambda})$ formam o vetor de parâmetro denotado por $\boldsymbol{\vartheta}=\left(\boldsymbol{\lambda}^{\top}, \boldsymbol{\varsigma}^{\top}\right)^{\top}$ (Masarotto et al., 2012).

Supondo que temos observações agrupadas em $m$ grupos de tamanho $n_{i}, i=1, \ldots, m$, em que $\sum_{i=1}^{m} n_{i}=n$. Sob a suposição de independência entre os diferentes grupos, matrizes de correlação adequadas para os erros são:

$$
\left[\begin{array}{cccc}
\boldsymbol{\Omega}_{1} & 0 & \ldots & 0 \\
0 & \boldsymbol{\Omega}_{2} & \ldots & 0 \\
\vdots & \vdots & \ddots & \vdots \\
0 & 0 & \ldots & \boldsymbol{\Omega}_{m}
\end{array}\right]
$$

em que $\boldsymbol{\Omega}_{i}$ é uma matriz de correlação $n_{i} \times n_{i}$. Cada $\boldsymbol{\Omega}_{i}$ pode ser especificado de forma similar ao método de EEGs como foi definido em (2.2.3).

No caso contínuo, a relação entre as respostas $y_{i}$ e erros $\epsilon_{i}$ produz uma expressão de forma fechada para a verossimilhança de $\boldsymbol{\vartheta}$. Por exemplo, para o caso bivariado temos o seguinte

$$
p_{i j}\left(y_{i}, y_{j} ; \boldsymbol{\vartheta}\right)=p_{i}\left(y_{i} ; \boldsymbol{\lambda}\right) p_{j}\left(y_{j} ; \boldsymbol{\lambda}\right) q\left(\epsilon_{i}, \epsilon_{j} ; \boldsymbol{\vartheta}\right),
$$

em que

$$
q\left(\epsilon_{i}, \epsilon_{j}, \boldsymbol{\vartheta}\right)=\frac{p\left(\epsilon_{i}, \epsilon_{j} ; \boldsymbol{\vartheta}\right)}{p\left(\epsilon_{i} ; \boldsymbol{\lambda}\right) p\left(\epsilon_{j} ; \boldsymbol{\lambda}\right)}
$$

é a função densidade de probabilidades bivariada da cópula Gaussiana. No geral a verossimilhança 
de $\boldsymbol{\vartheta}$ para o caso contínuo fica

$$
L(\boldsymbol{\vartheta} ; y)=\prod_{i=1}^{m} p_{i}\left(y_{i} ; \boldsymbol{\lambda}\right) q(\boldsymbol{\epsilon} ; \boldsymbol{\vartheta})
$$

em que a função densidade de probabilidade da cópula é

$$
q(\boldsymbol{\epsilon}, \boldsymbol{\vartheta})=\frac{p\left(\epsilon_{1}, \ldots, \epsilon_{m} ; \boldsymbol{\vartheta}\right)}{p\left(\epsilon_{1} ; \boldsymbol{\lambda}\right) \ldots p\left(\epsilon_{m} ; \boldsymbol{\lambda}\right)} .
$$

Para os casos discretos tem-se que as distribuições marginais conjuntas são expressas como integrais de normais multivariadas. Por exemplo, para o caso bivariado temos

$$
p_{i j}\left(y_{i}, y_{j} ; \boldsymbol{\vartheta}\right)=\int_{\mathcal{D}_{i}\left(y_{i} ; \boldsymbol{\lambda}\right)} \int_{\mathcal{D}_{j}\left(y_{j} ; \boldsymbol{\lambda}\right)} p\left(\epsilon_{i}, \epsilon_{j} ; \boldsymbol{\vartheta}\right) d \epsilon_{i} d \epsilon_{j},
$$

cujo domínio da integral é o produto cartesiano dos intervalos

$$
\mathcal{D}_{i}\left(y_{i} ; \boldsymbol{\lambda}\right)=\left[\Phi^{-1}\left(F_{i}\left(y_{i}^{-} ; \boldsymbol{\lambda}\right)\right), \Phi^{-1}\left(F_{i}\left(y_{i} ; \boldsymbol{\lambda}\right)\right)\right], i=1, \ldots, m,
$$

em que $F_{i}\left(y_{i}^{-} ; \lambda\right)$ é o limite inferior de $F_{i}(\cdot ; \lambda)$ em $y_{i} \operatorname{com} y_{i}^{-}=y_{i}-1$. Maiores detalhes podem ser encontrados em Masarotto et al. (2012). No caso geral de variáveis respostas discretas, a verossimilhança é dada pelo cálculo da seguinte integral

$$
L(\boldsymbol{\vartheta} ; y)=\int_{\mathcal{D}_{1}\left(y_{1} ; \boldsymbol{\lambda}\right)} \cdots \int_{\mathcal{D}_{m}\left(y_{m} ; \boldsymbol{\lambda}\right)} p\left(\epsilon_{1}, \ldots, \epsilon_{m} ; \boldsymbol{\vartheta}\right) d \epsilon_{1} \ldots d \epsilon_{m} .
$$

É conveniente reescrever a integral acima considerando a mudança da variável $\mathcal{D}_{1}\left(y_{1} ; \boldsymbol{\lambda}\right) \times \ldots \times$ $\mathcal{D}_{m}\left(y_{m} ; \boldsymbol{\lambda}\right)$ para $(0,1)^{m}$ de tal forma que

$$
\epsilon_{i}\left(u_{i}\right)=\Phi^{-1}\left\{F_{i}\left(y_{i} ; \boldsymbol{\lambda}\right)-u_{i} p_{i}\left(y_{i} ; \boldsymbol{\lambda}\right)\right\}, i=1, \ldots, m .
$$

Fazendo algumas mudanças de variáveis temos que a verossimilhança (3.12) pode assumir a forma

$$
L(\boldsymbol{\vartheta} ; y)=L_{i n d}(\boldsymbol{\lambda} ; y) \int_{[0,1]} \ldots \int_{[0,1]} q\left\{\epsilon_{1}\left(u_{1}\right), \ldots, \epsilon_{m}\left(u_{m}\right) ; \boldsymbol{\vartheta}\right\} d u_{1} \ldots d u_{m}
$$

em que $L_{i n d}(\boldsymbol{\lambda} ; y)$ é a verossimilhança considerando independência melhorada pela medida $q(\cdot)$. Para maiores detalhes ver Masarotto et al. (2012).

Como as funções que são usadas para ajustar modelos EEGs no R não tem suporte para respostas BN, a solução é utilizar a biblioteca gcmr. Esta biblioteca utiliza cópula Gaussianas para ajustar modelos marginais conforme descrito por Song (2000) e Masarotto et al. (2012) que permitem modelar respostas dependentes de qualquer tipo. O comando utilizado é

modelo $=\operatorname{gcmr}\left(y \sim x_{1}+x_{2}+x_{3}+\right.$ tempo, data $=$ dados, marginal $=$ negbin.marg, id $=i d$, cormat $=$ cluster . cormat $(i d$, type $="$ ") ).

A família da distribuição é identificada no objeto marginal e a matriz de correlação é informada no objeto type, dentro de cormat, cujas estruturas podem ser as mesmas definidas para as funções de EEG do R.

Trindade (2014) verificou que através dos exemplos apresentados no manual, os resultados das estimativas usando as bibliotecas EEG são similares aos da função gcmr. Porém, o critério de seleção utilizado em cada método é distinto, uma vez que as bibliotecas EEG considera o QIC e a biblioteca de cópulas Gaussianas utiliza o AIC.

Em http://cran.r-project.org/web/packIdades/gcmr/gcmr.pdf é possível encontrar maiores detalhes sobre o pacote $\mathrm{gcmr}$.

No final da dissertação da Trindade (2014), quando são feitas as comparações entre os modelos, foi concluído que os dados ajustados por modelos de cópulas Gaussianas com resposta binomial 
negativa não convergiam para estruturas complexas, com grande quantidade de réplicas ou de observações. Portanto, nem sempre é uma boa solução. 


\section{Capítulo 4}

\section{Aplicações}

A ideia agora é aplicar os conhecimentos que foram discutidos anteriormente para os diversos tipos de dados que já foram discutidos no Capítulo 1. Para essas análises será considerado um valor do nível de significância de 10\%. Além disso por estarmos trabalhando com dados que possivelmente não são simétricos, vamos utilizar um boxplot robusto que corrige esse viés. O boxplot é feito através do pacote rosbustbase. Nesses boxplots teremos a linha da mediana, que é a preta mais grossa, e a da média que é a cinza mais fina.

\subsection{Pacientes com Lepra}

\subsubsection{Análise Preliminar dos Dados}

Primeiramente vamos ver como se comportam os dados de uma forma geral.

Tabela 4.1: Medidas resumo da contagem de bacilos.

\begin{tabular}{r|r}
\hline Média & Variância \\
\hline 9,317 & 35,169 \\
\hline
\end{tabular}

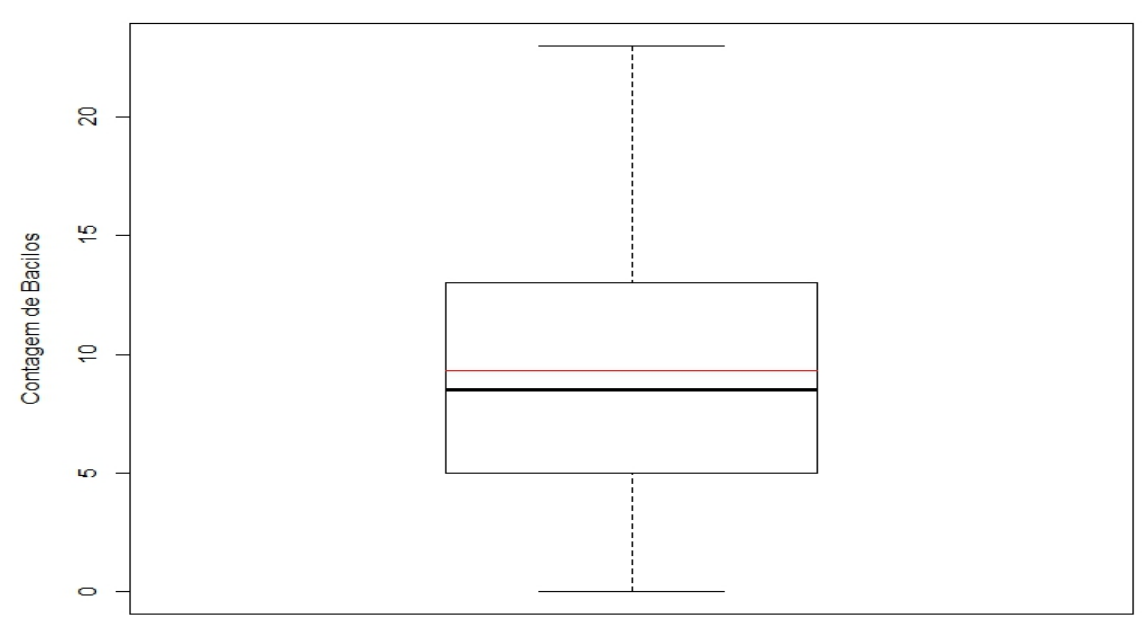

Figura 4.1: Boxplot robusto da contagem de bacilos. 
Agora vamos verificar como se comportam os dados quando são separados por tratamento, ou seja, em um grupo que recebeu placebo e o grupo que recebeu a droga.

Tabela 4.2: Medidas resumo da contagem de bacilos segundo o tratamento.

\begin{tabular}{c|r|r}
\hline Tipo & Média & Variância \\
\hline Placebo & 7,300 & 25,168 \\
Tratamento & 10,325 & 37,815 \\
\hline
\end{tabular}

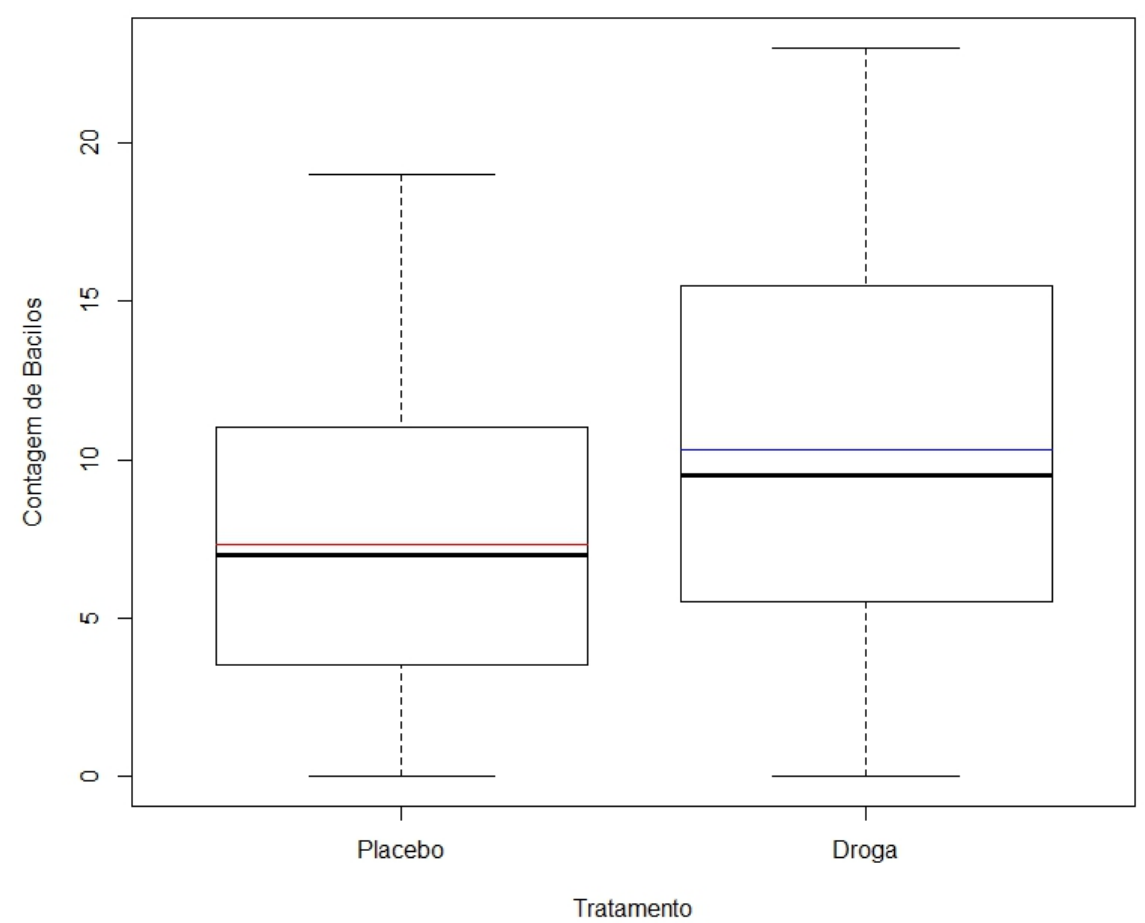

Figura 4.2: Boxplot robusto da contagem de bacilos segundo o tratamento.

Vemos que a média da quantidade de bacilos no grupo placebo é menor do que no grupo tratado, e apesar de o grupo que recebeu a droga possuir uma variância maior, o comportamento dos grupos parece similar pelos dois boxplots. O que pode indicar que a droga não influencia na contagem de bacilos ou que influencia de forma contrária, aumenta a quantidade de bacilos. 
Agora vamos comparar os dados separando-os pelo momento da medida, ou seja, se foi antes de começarem o tratamento ou depois.

Tabela 4.3: Medidas resumo da contagem de bacilos segundo o momento, antes ou depois do tratamento.

\begin{tabular}{c|r|r}
\hline Momento & Média & Variância \\
\hline Antes & 10,733 & 22,961 \\
Depois & 7,900 & 44,438 \\
\hline
\end{tabular}

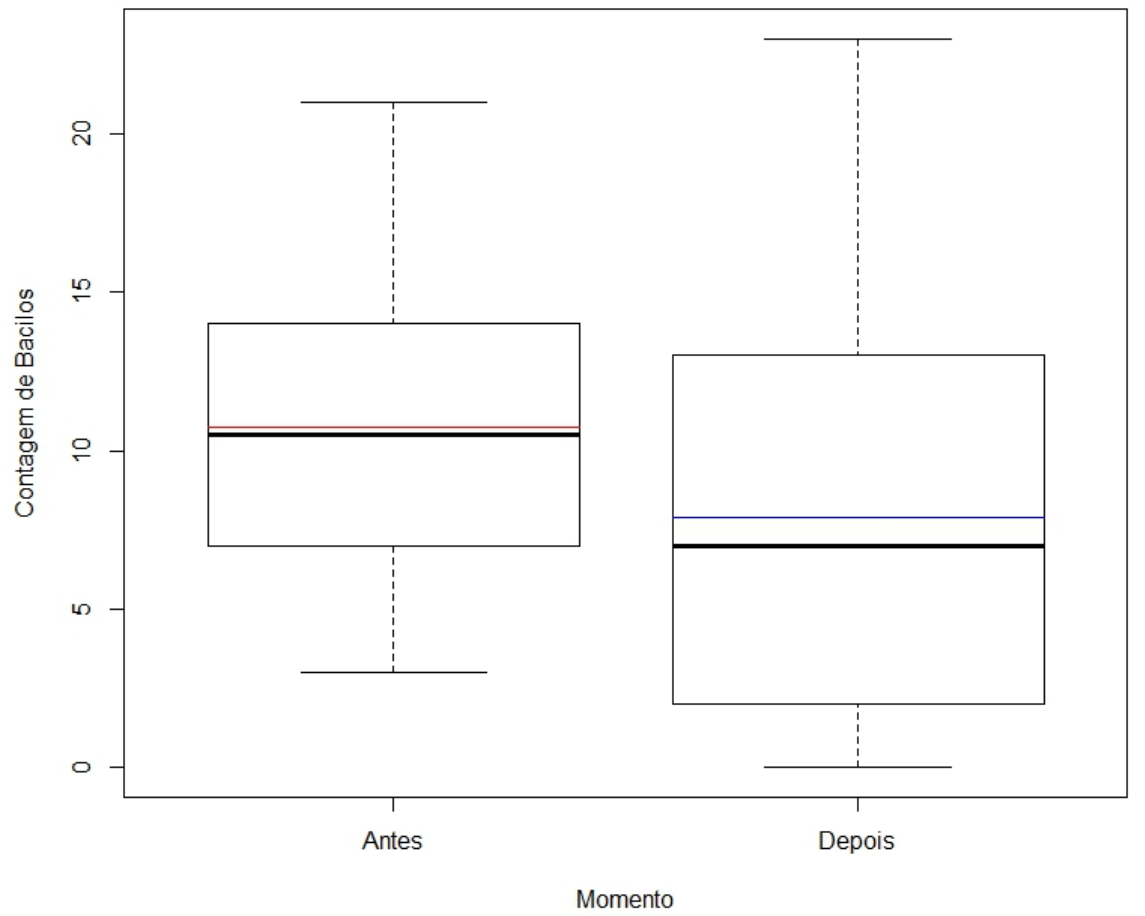

Figura 4.3: Boxplot robusto da contagem de bacilos segundo o momento.

Pelos resultados podemos notar que a média da quantidade de bacilos diminui após o tratamento. Contudo, a variância da contagem aumenta. 
A seguir vamos comparar os dados separados pelos 2 grupos, tratamento e momento.

Tabela 4.4: Medidas resumo da contagem de bacilos segundo tratamento e momento.

\begin{tabular}{c|r|r|r|r}
\hline Tratamento & \multicolumn{2}{|c}{ Placebo } & \multicolumn{2}{c}{ Droga } \\
\hline Momento & Média & Variância & Média & Variância \\
Antes & 9,300 & 22,678 & 11,450 & 22,682 \\
Depois & 5,300 & 21,567 & 9,200 & 52,274 \\
\hline
\end{tabular}

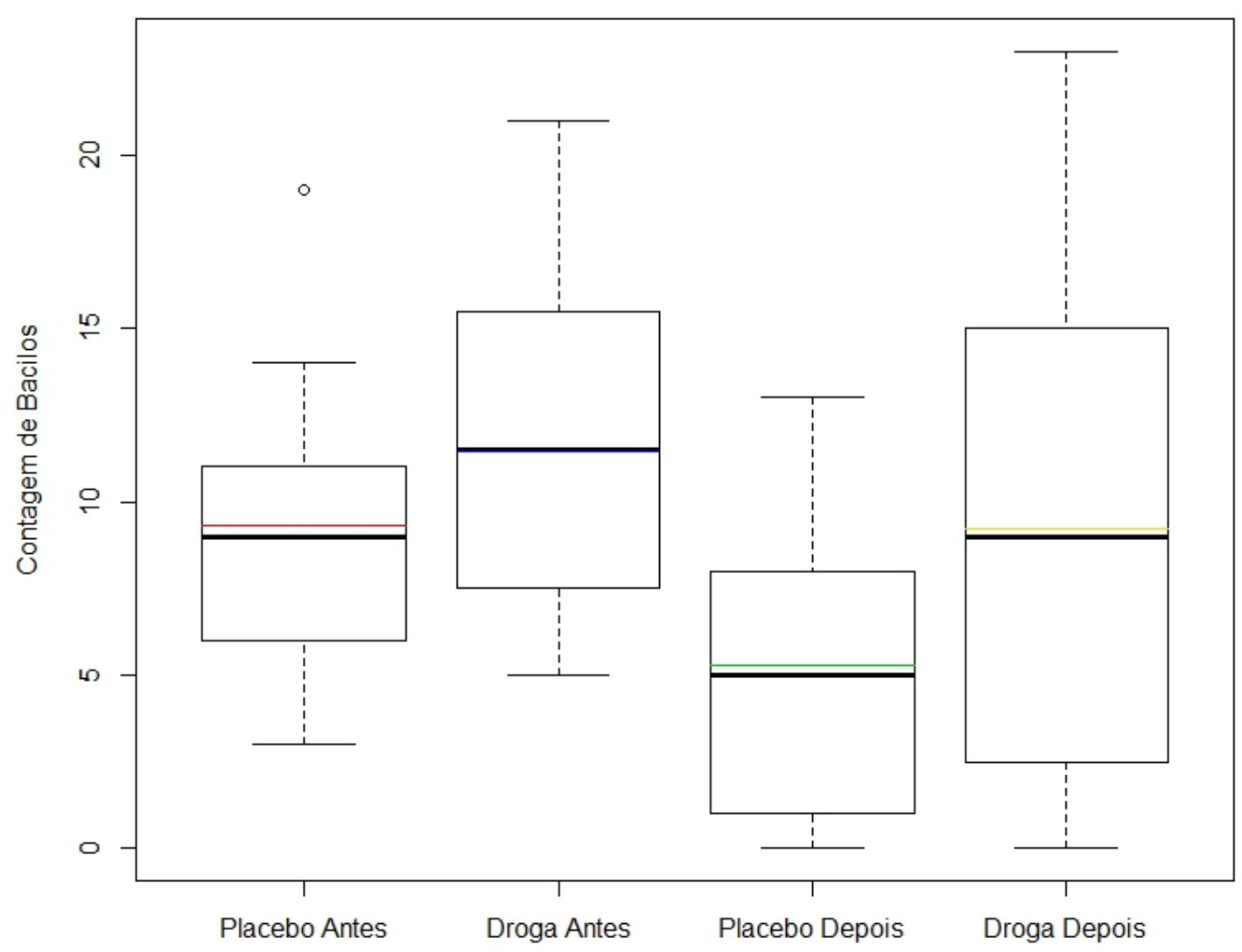

Figura 4.4: Boxplot robusto da contagem de bacilos segundo o tratamento e o momento.

Podemos notar que antes do tratamento começar o grupo placebo já possuía uma média de quantidade de bacilos menor do que os pacientes do grupo com a droga. Depois que o tratamento é aplicado ambos os grupos apresentaram uma redução na quantidade de bacilos. No entanto, enquanto o grupo placebo diminuiu a variância, o grupo da droga aumentou bastante a variância. $\mathrm{O}$ que isso pode sugerir é que receber o tratamento influencia na diminuição da quantidade de bacilos. Entre a droga e o placebo, parece que a droga gerou resultados piores.

O banco de dados Leprosy foi descrito no artigo de Cui e Feng (2008), onde é apresentada uma análise para encontrar a melhor estrutura de correlação e seleção de modelos através do QIC. No artigo o modelo final obtido é o modelo EEG - BN com estrutura de correlação simétrica e considerando a variável tempo como a única significativa no modelo. Vamos modelar esse mesmo banco de dados no $\mathrm{R}$ e no SAS. Como o artigo trabalha com o STATA vamos verificar se as conclusões são similares. 


\subsubsection{Selecionando o Modelo}

Vamos ajustar alguns modelos para verificar qual a melhor forma de explicar a quantidade média de bacilos. Denotaremos:

- $y_{i j}$ : a quantidade de bacilos do paciente $i$ no momento $j$;

- $x_{1 i}$ : o momento em que ocorreu a medida da quantidade de bacilos do paciente $i\left(x_{1 i}=0\right.$ antes, $x_{1 i}=1$ depois);

- $x_{2 i}$ : indicador de qual grupo o $i$-ésimo paciente pertencia $\left(x_{2 i}=0\right.$ placebo, $x_{2 i}=1$ tratamento).

Primeiramente, verificaremos se um modelo linear normal ajusta bem aos dados de pacientes com lepra. Vamos considerar então:

$$
y_{i j}=\beta_{0}+\beta_{1} x_{1 i}+\beta_{2} x_{2 i}+\beta_{3}\left(x_{1 i} \times x_{2 i}\right)+\epsilon_{i j}, \quad i=1, \ldots, 30, \quad \text { e } \quad j=0,1,
$$

com a suposição de que $\epsilon_{i j} \stackrel{i i d}{\sim} \mathrm{N}\left(0, \sigma^{2}\right)$. O resultado da estimação dos parâmetros do modelo ajustado se encontra na Tabela 4.5.

Tabela 4.5: Estimativas dos parâmetros do modelo linear normal ajustado no $R$ aos dados de pacientes com lepra.

\begin{tabular}{c|r|r|r|r}
\hline Efeito & Estimativa & Erro Padrão & Valor $-\mathrm{z}$ & Valor - p \\
\hline Intercepto & 9,300 & 1,804 & 5,160 & $<0,001$ \\
Depois & $-4,000$ & 2,551 & $-1,570$ & 0,123 \\
Droga & 2,150 & 2,209 & 0,970 & 0,335 \\
Depois:Droga & 1,750 & 3,125 & 0,560 & 0,578 \\
\hline
\end{tabular}

O $R^{2}$ ajustado foi encontrado no valor de 0,075 , que é muito baixo, o que era de esperar considerando que a variável resposta não é normal. Além disso, nenhum dos efeitos é marginalmente significativo. Como a variável resposta é a contagem de bacilos do paciente em um dado momento, podemos supor que a resposta segue uma distribuição de Poisson. Ajustaremos então um modelo com resposta de Poisson, com as seguintes suposições:

- $y_{i j} \stackrel{i n d}{\sim} \operatorname{Poisson}\left(\mu_{i j}\right), i=1, \ldots, 30$ e $j=0,1$;

- $\log \left(\mu_{i j}\right)=\beta_{0}+\beta_{1} x_{1 i}+\beta_{2} x_{2 i}+\beta_{3}\left(x_{1 i} \times x_{2 i}\right)$.

Os resultados do modelo ajustado são apresentados na Tabela 4.6.

Tabela 4.6: Estimativas dos parâmetros do modelo log-linear de Poisson ajustado no $R$ aos dados dos pacientes com lepra.

\begin{tabular}{c|r|r|r|r}
\hline Efeito & Estimativa & Erro Padrão & Valor - z & Valor - p \\
\hline Intercepto & 2,230 & 0,104 & 21,510 & $<0,001$ \\
Depois & $-0,562$ & 0,172 & $-3,270$ & 0,001 \\
Droga & 0,208 & 0,123 & 1,690 & 0,091 \\
Depois:Droga & 0,344 & 0,199 & 1,730 & 0,084 \\
\hline
\end{tabular}

O AIC obtido com esse modelo foi de 456,700. O próximo passo é verificar se esse modelo se ajusta bem aos dados. Através de uma análise de diagnóstico para verificar se as nossas suposições sobre o modelo não estão incorretas. O desvio do modelo foi de 225, 53 (56 graus de liberdade). 
Os gráficos abaixo foram construídos com os comandos encontrados no site: http://www.ime. usp.br/〜 giapaula/cursospos.htm.
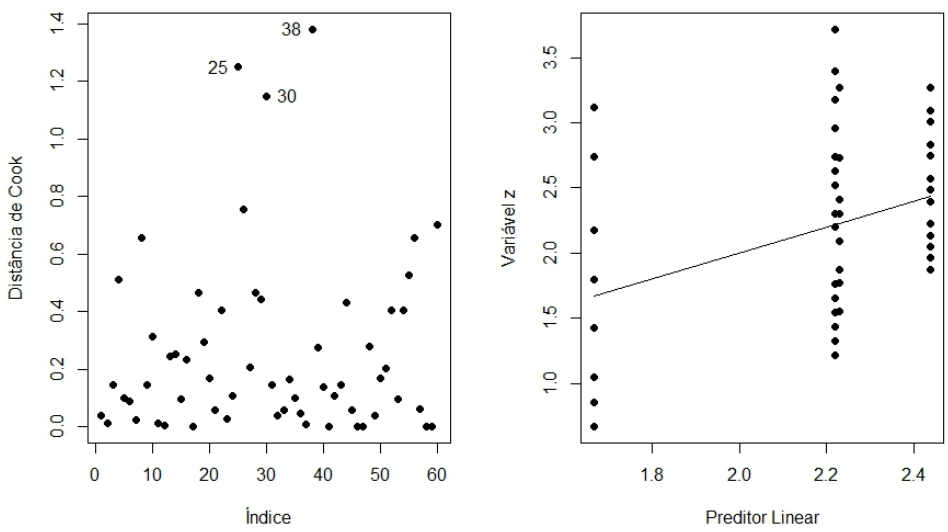

Figura 4.5: Gráficos de diagnóstico referentes ao ajuste no $R$ do modelo log-linear de Poisson aos dados de pacientes com lepra.

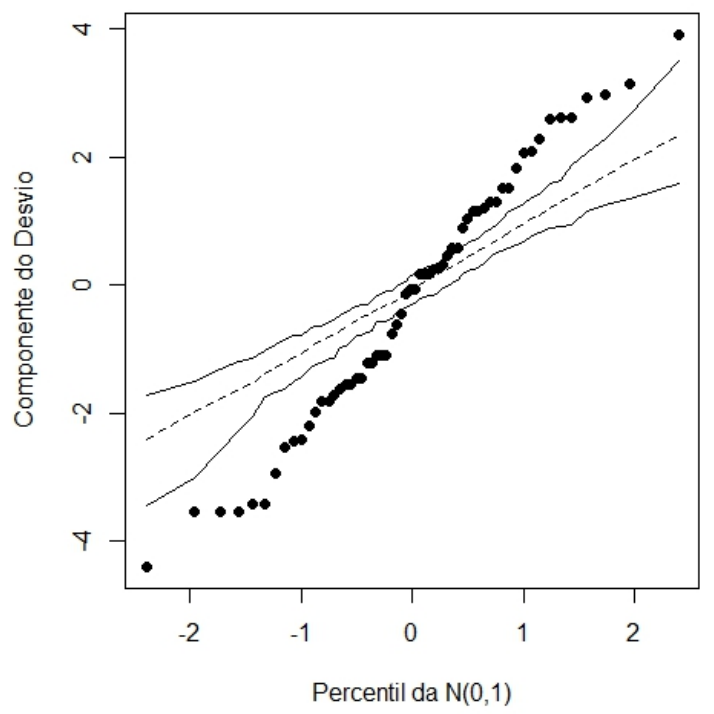

Figura 4.6: Gráfico normal de probabilidades referente aos ajuste no $R$ do modelo log-linear de Poisson aos dados de pacientes com lepra.

Pelos gráficos de diagnóstico da Figura 4.5 notamos três observações com mais destaque e a confirmação de que a ligação logarítmica não é inadequada. Todavia, o gráfico normal de probabilidades da Figura 4.6 indica sobredispersão, confirmando resultados anteriores, e sugerindo um modelo com variância maior do que a média, por exemplo, binomial negativo. 
Vamos supor então o seguinte modelo com resposta binomial negativa:

- $y_{i j} \stackrel{i n d}{\sim} \mathrm{BN}\left(\mu_{i j}, \nu\right), i=1, \ldots, 30$ e $j=0,1$;

- $\log \left(\mu_{i j}\right)=\beta_{0}+\beta_{1} x_{1 i}+\beta_{2} x_{2 i}+\beta_{3}\left(x_{1 i} \times x_{2 i}\right)$.

Tabela 4.7: Estimativas dos parâmetros do modelo log-linear binomial negativo ajustado no $R$ aos dados de pacientes com lepra.

\begin{tabular}{c|r|r|r|r}
\hline Efeito & Estimativa & Erro Padrão & Valor - z & Valor - p \\
\hline Intercepto & 2,230 & 0,213 & 10,480 & $<0,001$ \\
Depois & $-0,562$ & 0,314 & $-1,790$ & 0,073 \\
Droga & 0,208 & 0,259 & 0,800 & 0,421 \\
Depois:Droga & 0,344 & 0,378 & 0,910 & 0,364 \\
$\hat{\nu}$ & 2,896 & 0,767 & & \\
\hline
\end{tabular}

Com um AIC de 383,930, temos que o modelo está melhor ajustado do que o modelo com resposta Poisson. O desvio do modelo foi de 70,55 (56 graus de liberdade) também sugerindo um ajuste mais adequado. Agora veremos como o modelo se encontra na análise de diagnóstico.
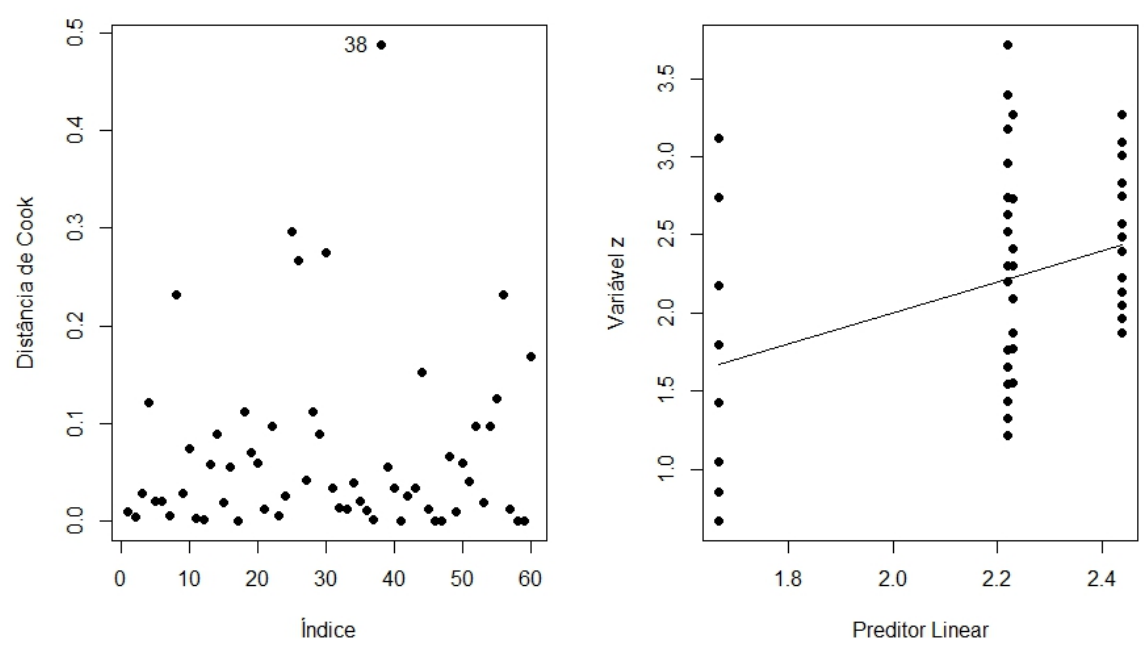

Figura 4.7: Gráficos de diagnóstico referentes ao ajuste no $R$ do modelo log-linear binomial negativo aos dados de pacientes com lepra. 


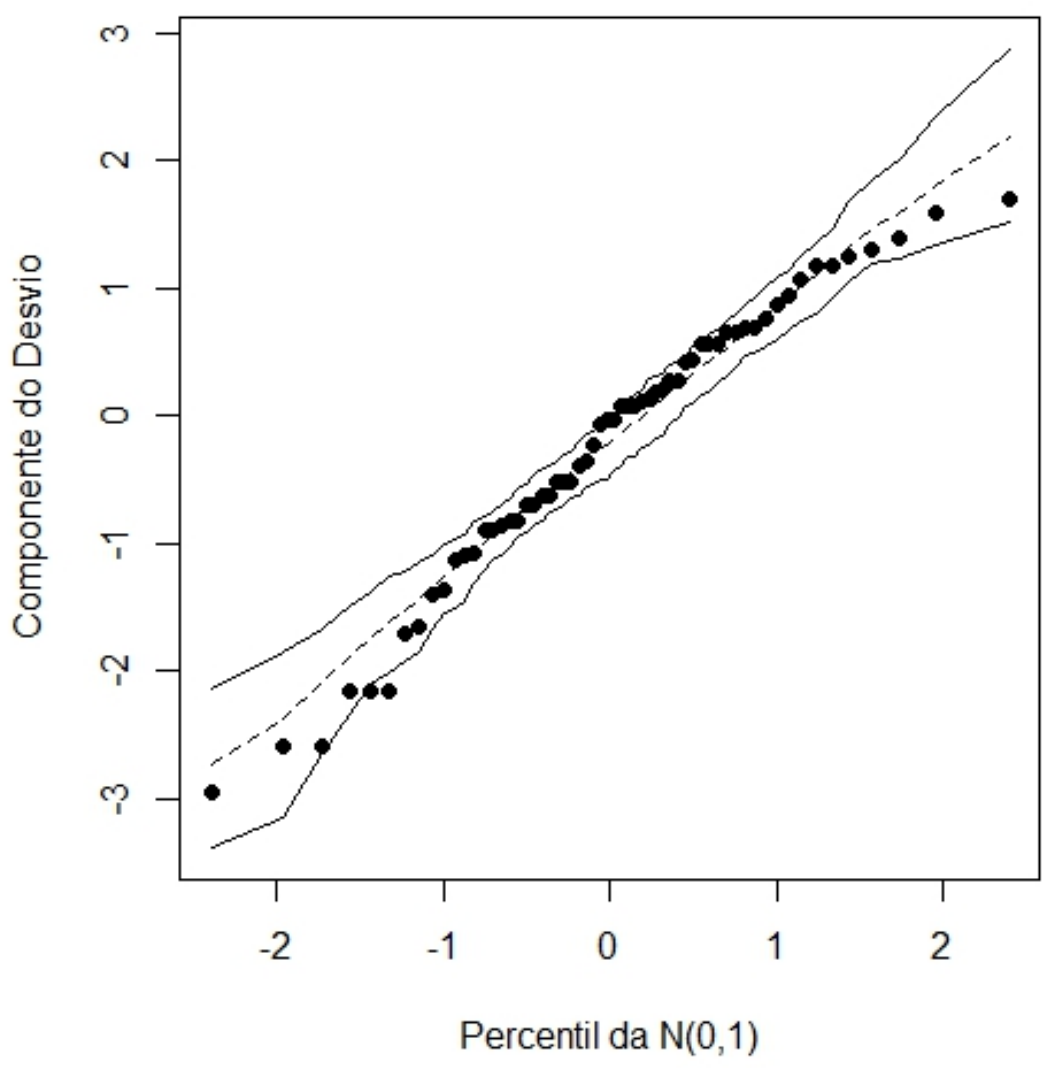

Figura 4.8: Gráfico normal de probabilidades referente ao ajuste no $R$ do modelo log-linear binomial negativo aos dados de pacientes com lepra.

Podemos notar pelos gráficos de diagnóstico um ajuste melhor sob o modelo com resposta binomial negativa. Podemos também concluir que não foi possível detectar interação entre momento e tratamento. Contudo, originalmente, os dados têm estrutura longitudinal (as medidas de um mesmo paciente foram feitas em dois momentos) e pode ser que o fato dessa estrutura não ter sido levada em conta possa ter afetado na detectação de alguns efeitos. Vamos então considerar a seguir os mesmos modelos, porém com alguma estrutura de correlação.

Inicialmente vamos ajustar pelo $\mathrm{R}$ uma EEG com resposta Poisson, já que nossa variável resposta é uma contagem, com o agrupamento por paciente. O modelo proposto fica dado por

- $y_{i j} \sim \operatorname{Poisson}\left(\mu_{i j}\right), i=1, \ldots, 30$ e $j=0,1$;

- $\log \left(\mu_{i j}\right)=\beta_{0}+\beta_{1} x_{1 i}+\beta_{2} x_{2 i}+\beta_{3}\left(x_{1 i} \times x_{2 i}\right)$;

- $\operatorname{Corr}\left(y_{i j}, y_{i j^{\prime}}\right)=\rho$ para $j \neq j^{\prime}$ e $\operatorname{Corr}\left(y_{i j}, y_{i j^{\prime}}\right)=1$ para $j=j^{\prime}$.

Ajustando o modelo acima obtemos os resultados descritos na Tabela 4.8. 
Tabela 4.8: Estimativas dos parâmetros referentes ao modelo EEG-Poisson ajustado no $R$ aos dados de pacientes com lepra.

\begin{tabular}{c|r|r|r|r}
\hline Efeito & Estimativa & Erro Padrão & Valor-z & Valor-p \\
\hline Intercepto & 2,230 & 0,154 & 14,520 & $<0,001$ \\
Depois & $-0,562$ & 0,176 & 3,195 & 0,001 \\
Droga & 0,208 & 0,178 & 1,166 & 0,244 \\
Depois:Droga & 0,344 & 0,209 & 1,643 & 0,100 \\
Escala & 3,403 & 0,499 & & \\
\hline
\end{tabular}

Utilizando a função QIC do pacote MuMIn no R encontramos que o QIC do modelo é de -1400.
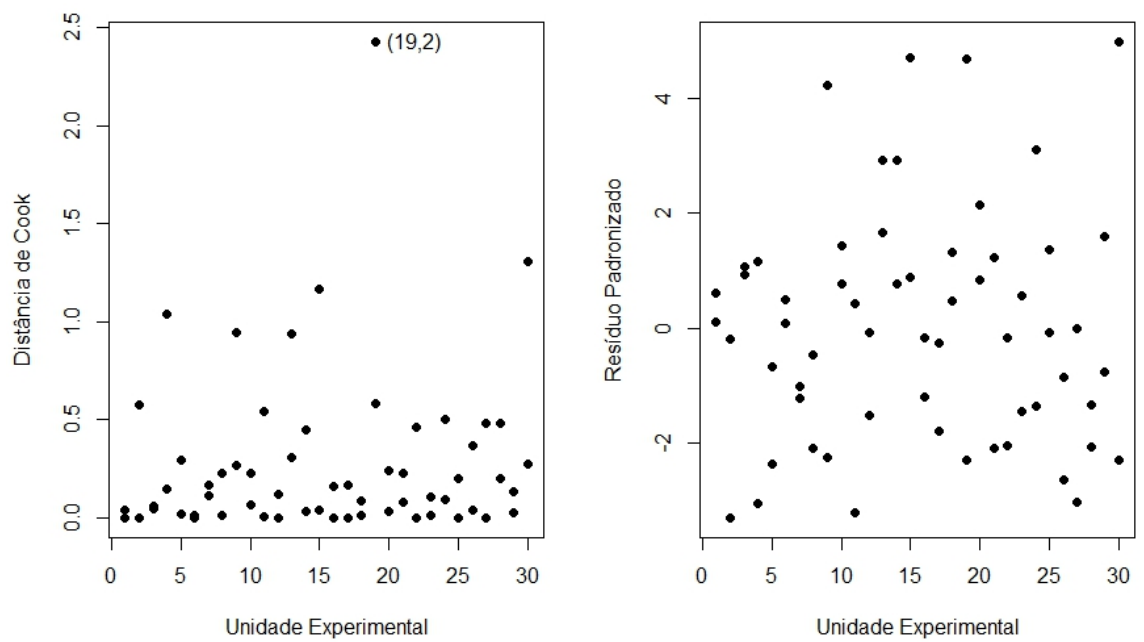

Figura 4.9: Métodos de diagnóstico referentes ao ajuste no $R$ do modelo EEG-Poisson aos dados de pacientes com lepra.

Nota-se pelos gráficos de diagnóstico uma observação influente e também uma quantidade grande de unidades experimentais com resíduos (em valor absoluto) acima de 2, um indício de sobredispersão. Como foi discutido no texto, não foi encontrada nenhuma função ou pacote no $R$ que ajuste EEGs sob resposta binomial negativa, portanto essa análise será feita com o programa SAS. Para o modelo EEG-Poisson os códigos são dados por:

PROC GENMOD DATA=WORK. LEPROSY

PLOTS $(O N L Y)=$ ALL;

CLASS ID id;

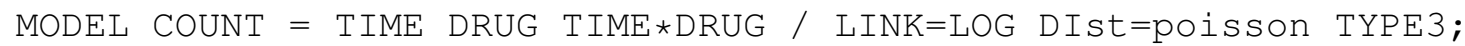

REPEATED SUBJECT=ID / SORTED WITHINSUBJECT=id TYPE=EXCH COVB CORRW MODELSE; RUN;

Já para o modelo EEG-BN os códigos são os seguintes:

PROC GENMOD DATA=WORK. LEPROSY

PLOTS $(O N L Y)=$ ALL;

CLASS ID id;

MODEL COUNT = TIME DRUG TIME $\star$ DRUG / LINK=LOG DISt=negbin TYPE3;

REPEATED SUBJECT=ID / SORTED WITHINSUBJECT=id TYPE=EXCH COVB CORRW MODELSE; RUN; 
Resultado que foi obtido no SAS para o modelo EEG-Poisson é descrito na Tabela 4.9.

Tabela 4.9: Estimativas dos parâmetros do modelo EEG-Poisson ajustado no SAS aos dados de pacientes com lepra.

\begin{tabular}{c|r|r|r|r|r|r}
\hline Efeito & Estimativa & Erro Padrão & \multicolumn{2}{|c|}{ IC 95\% } & Valor-z & Valor-p \\
\hline Intercept & 2,230 & 0,198 & 1,842 & 2,618 & 11,260 & $<0,001$ \\
Depois & $-0,562$ & 0,169 & $-0,893$ & $-0,232$ & $-3,340$ & 0,001 \\
Droga & 0,208 & 0,235 & $-0,252$ & 0,668 & 0,890 & 0,376 \\
Depois:Droga & 0,344 & 0,192 & $-0,033$ & 0,720 & 1,790 & 0,074 \\
$\sqrt{\text { Escala }}$ & 1,909 &. &. &. &. &. \\
\hline
\end{tabular}

O QIC encontrado foi no valor de -378,673 e matriz de correlação estimada é apresentada na Tabela 4.10.

Tabela 4.10: Estimativa da matriz trabalho referente ao ajuste no SAS do modelo EEG-Poisson aos dados de pacientes com lepra.

$$
\left[\begin{array}{cc}
1 & 0,766 \\
0,766 & 1
\end{array}\right]
$$

Os estimativas dos parâmetros são iguais aos que foram encontrados pelo R, e as conclusões são as mesmas apesar de algumas diferenças no valor-p.

Ajustando agora o modelo EEG-BN, temos o resultado obtido pelo SAS descrito na Tabela 4.11.

Tabela 4.11: Estimativa dos parâmetros do modelo EEG-BN ajustado no SAS aos dados de pacientes com lepra.

\begin{tabular}{c|r|r|r|r|r|r}
\hline Efeito & Estimativa & Erro Padrão & \multicolumn{2}{|c|}{ IC 95\% } & Valor-z & Valor-p \\
\hline Intercepto & 2,230 & 0,204 & 1,830 & 2,630 & 10,920 & $<0,001$ \\
Depois & $-0,562$ & 0,158 & $-0,871$ & $-0,254$ & $-3,570$ & $<0,001$ \\
Droga & 0,208 & 0,248 & $-0,279$ & 0,695 & 0,840 & 0,402 \\
Depois:Droga & 0,344 & 0,189 & $-0,028$ & 0,715 & 1,810 & 0,070 \\
$\sqrt{\text { Escala }}$ & 0,960 &. &. &. &. &. \\
\hline
\end{tabular}

Com QIC de -1600, 414 e matriz de correlação estimada é apresentada na Tabela 4.12.

Tabela 4.12: Estimativa da matriz trabalho referente ao ajuste no SAS do modelo EEG-BN aos dados de pacientes com lepra.

$$
\left[\begin{array}{cc}
1 & 0,7298 \\
0,7298 & 1
\end{array}\right]
$$

A partir das características dos dados e do QIC podemos concluir que um modelo EEG-BN é o que melhor se ajusta aos dados. Contudo, outro aspecto que devemos levar em conta quando trabalhamos com EEGs é descobrir qual a melhor matriz de correlação que reflete os dados.

Para esse conjunto de dados foram testadas as outras estruturas de correlação disponíveis, tais como a simétrica, independente, $\operatorname{AR}(1)$ e não-estruturada, e todas retornaram o mesmo valor de QIC. Assim, como é explicado em Cui e Feng (2008), isso ocorre porque temos apenas dois pontos temporais para cada indivíduo. Logo, como no artigo, vamos escolher como a melhor estrutura de correlação a simétrica. 


\subsubsection{Interpretações}

Portanto, a estimativa para o valor esperado para a quantidade de bacilos sob o modelo EEG-BN fica dada por:

$$
\hat{\mu}\left(x_{1}, x_{2}\right)=\exp \left\{2,230-0,562 \times x_{1}+0,208 \times x_{2}+0,344 \times x_{1} \times x_{2}\right\},
$$

para $i=1, \ldots, 30$ e $j=0,1, \operatorname{com} x_{1}(=0$ antes, $=1$ depois $)$ e $x_{2}(=0$ placebo, $=1$ droga $)$. Temos portanto quatro médias estimadas $\hat{\mu}(0,0)=\exp (2,230)=9,30, \hat{\mu}(0,1)=\exp (2,230+0,208)=$ $11,45, \hat{\mu}(1,0)=\exp (2,230-0,562)=5,30$ e $\hat{\mu}(1,1)=\exp (2,230-0,562+0,208+0,344)=9,21$. Portanto, a maior redução no número médio de bacilos ocorre no grupo placebo após o tratamento. Para o grupo que recebeu a droga também há uma redução no número médio predito de bacilos após o tratamento. Esses resultados confirmam a análise descritiva.

\subsubsection{Método Alternativo}

Para fins de comparação iremos avaliar se os resultados encontrados pelo modelo EEG-BN no SAS são similares com os resultados encontrados através de cópulas Gaussianas no R. Para essa comparação vamos supor o modelo de cópulas Gaussianas com respostas Poisson e binomial negativa, respectivamente.

Tabela 4.13: Estimativas dos parâmetros através do ajuste do modelo de cópulas Gaussianas com resposta Poisson ajustado no $R$ aos dados de pacientes com lepra.

\begin{tabular}{c|r|r|r|r}
\hline Efeito & Estimativa & Erro Padrão & Valor - z & Valor - p \\
\hline Intercepto & 2,249 & 0,102 & 21,95 & $<0,001$ \\
Depois & $-0,562$ & 0,314 & $-1,79$ & $<0,001$ \\
Droga & 0,212 & 0,121 & 1,75 & 0,081 \\
Depois:Droga & 0,342 & 0,144 & 2,38 & 0,017 \\
\hline
\end{tabular}

Com AIC de 419,600.

Tabela 4.14: Estimativas dos prâmetros através do ajuste do modelo de cópulas Gaussianas com resposta binomial negativa ajustado no $R$ aos dados de pacientes com lepra.

\begin{tabular}{c|r|r|r|r}
\hline Efeito & Estimativa & Erro Padrão & Valor - z & Valor - p \\
\hline Intercepto & 2,257 & 0,199 & 11,32 & $<0,001$ \\
Depois & $-0,615$ & 0,193 & $-3,19$ & $<0,001$ \\
Droga & 0,211 & 0,242 & 0,87 & 0,383 \\
Depois:Droga & 0,293 & 0,231 & 1,46 & 0,144 \\
$\hat{\nu}$ & 0,293 & 0,093 & & \\
\hline
\end{tabular}

Com AIC de 370, 300.

Portanto, podemos notar que as estimativas obtidas através do modelo de cópulas Gaussianas com resposta binomial negativa (que apresenta um AIC menor) apresenta valores pontuais similares aos do ajuste do modelo EEG-BN, porém a inferência não é a mesma. Não foi detectado pelo modelo de cópulas Gaussianas o efeito de interação entre tratamento e momento, ao nível de significância de $10 \%$. 


\subsection{Pacientes Epilépticos}

\subsubsection{Análise Preliminar dos Dados}

Fazendo uma análise de como se comporta a variável quantidade de ataques epilépticos, obtemos os resultados descritos abaixos.

Tabela 4.15: Medidas resumo da contagem de ataques epilépticos.

\begin{tabular}{r|r}
\hline Média & Variância \\
\hline 12,864 & 349,240 \\
\hline
\end{tabular}

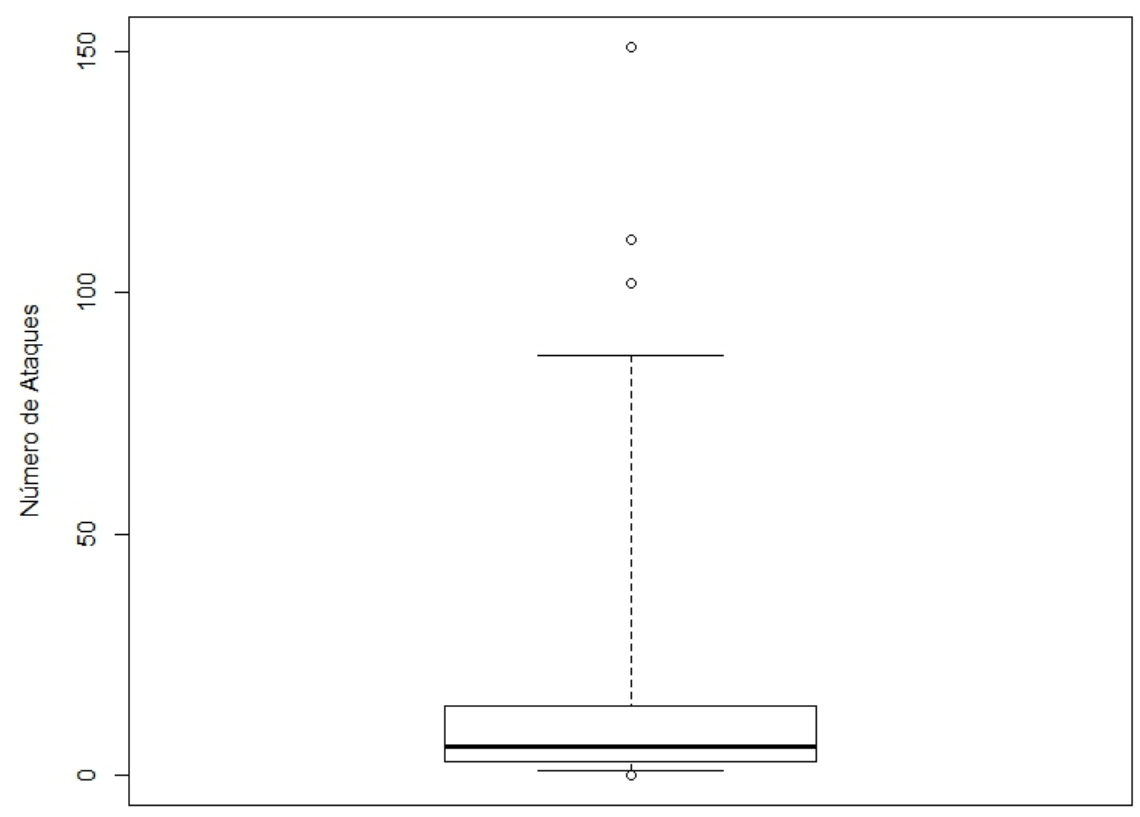

Figura 4.10: Boxplot robusto do número de ataques epilépticos.

Vemos que a média e a variância são bem diferentes, e pelo boxplot percebemos a maior concentração dos valores entre 0 e 50 .

Abaixo descrevemos o comportamento dos dados quando são classificados segundo o tratamento.

Tabela 4.16: Medidas resumo da contagem de ataques epilépticos segundo o tratamento.

\begin{tabular}{c|r|r}
\hline Tipo & Média & Variância \\
\hline Placebo & 13,043 & 297,826 \\
Progabide & 12,703 & 397,859 \\
\hline
\end{tabular}

As médias aparentemente são bem próximas, apesar de os valores do grupo tratado com Progabide ter uma variância maior. Isso também pode ser observado no boxplot descrito na Figura 4.11 . 


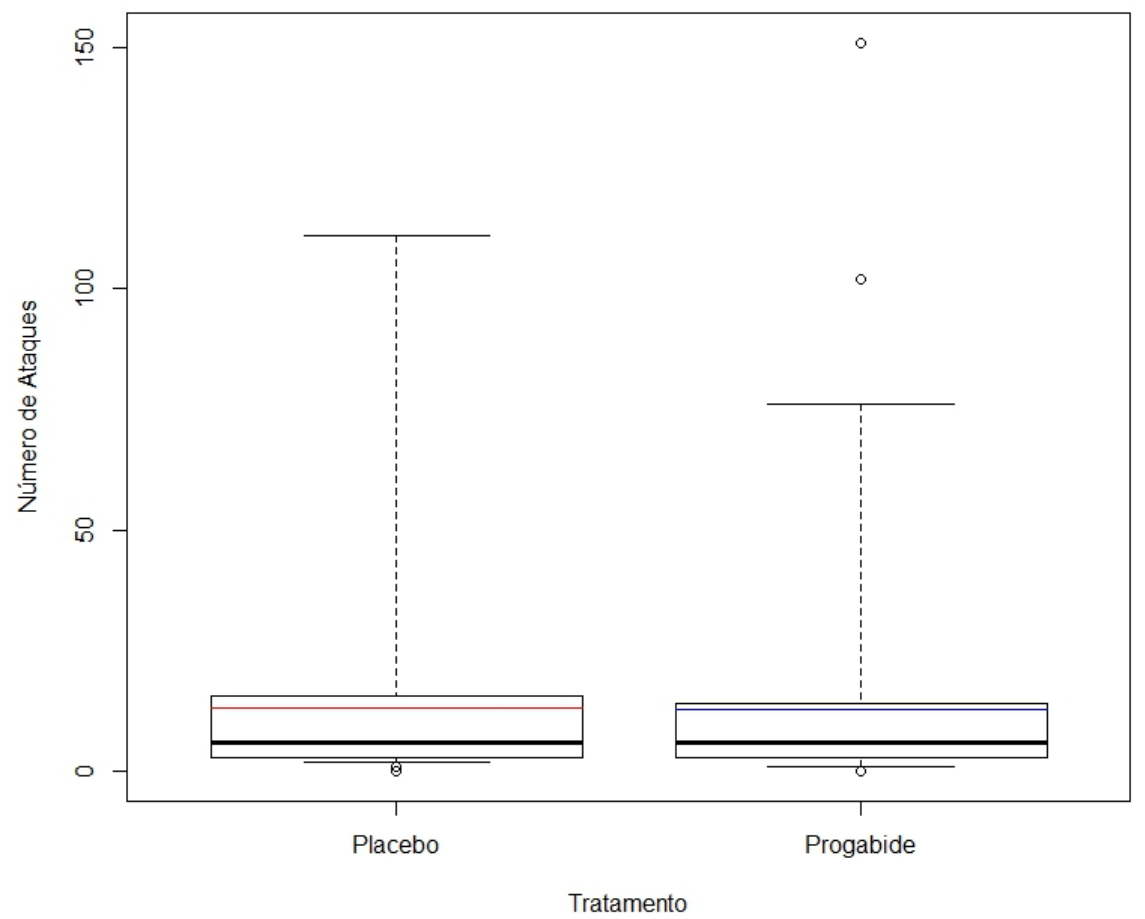

Figura 4.11: Boxplot robusto do número de ataques epilépticos segundo o tratamento.

Quando comparamos os valores do número de ataques epilépticos por período obtemos os resultados descritos na Tabela 4.17 .

Tabela 4.17: Medidas resumo da contagem de ataques epilépticos por periodo.

\begin{tabular}{c|r|r}
\hline Momento & Média & Variância \\
\hline Base ( 8 semanas) & 31,237 & 722,736 \\
1 (2 semanas) & 8,949 & 220,084 \\
2 (2 semanas) & 8,356 & 103,785 \\
3 (2 semanas) & 8,441 & 200,182 \\
4 (2 semanas) & 7,339 & 92,883 \\
\hline
\end{tabular}

Não podemos comparar os valores do momento Base com os outros períodos pois no período base a duração foi maior. Mas, ao compararmos os valores nos 4 períodos de 2 semanas cada notamos uma queda na média de ataques epilépticos, bem como da variância. Contudo, a relação variância/média continua alta. 


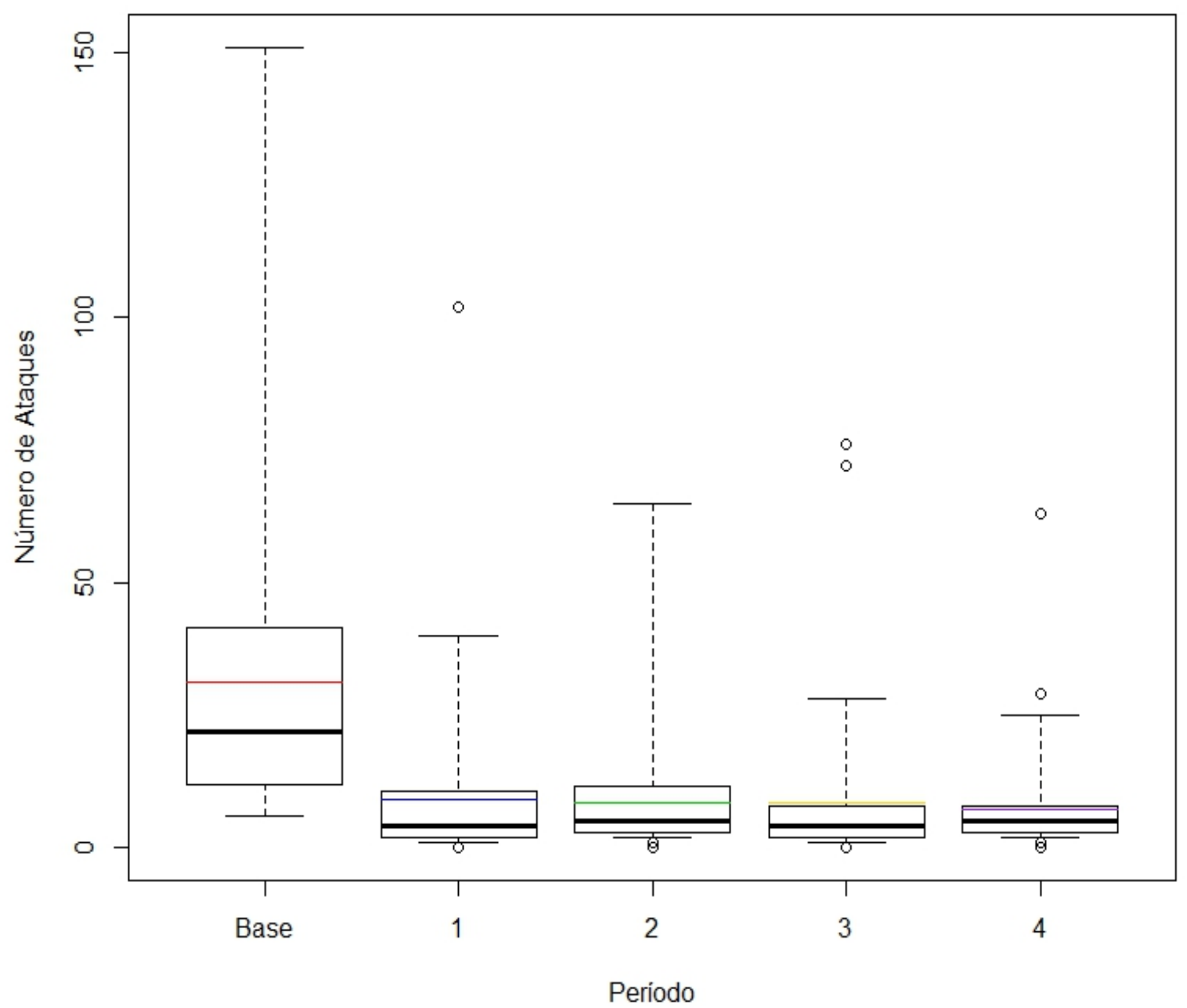

Figura 4.12: Boxplot robusto do número de ataques epilépticos segundo o período.

É importante verificar como a quantidade de ataques epilépticos se comporta segundo o período e o tratamento. Essa comparação é descrita na Tabela 4.18.

Tabela 4.18: Medidas resumo do número de ataques epilépticos segundo o periodo e o tratamento.

\begin{tabular}{c|r|r|r|r}
\hline Tratamento & \multicolumn{2}{|c}{ Placebo } & \multicolumn{2}{c}{ Progabide } \\
\hline Momento & Média & Variância & Média & Variância \\
\hline Base (8 semanas) & 30,786 & 681,434 & 31,645 & 783,637 \\
1 (2 semanas) & 9,357 & 102,757 & 8,851 & 332,718 \\
2 (2 semanas) & 8,286 & 66,656 & 8,419 & 140,652 \\
3 (2 semanas) & 8,786 & 215,286 & 8,129 & 193,049 \\
4 (2 semanas) & 8,000 & 57,926 & 6,742 & 126,665 \\
\hline
\end{tabular}

Pela Tabela 4.18 notamos que o tratamento com Progabide levou a uma redução da média de ataques epilépticos com o tempo. 


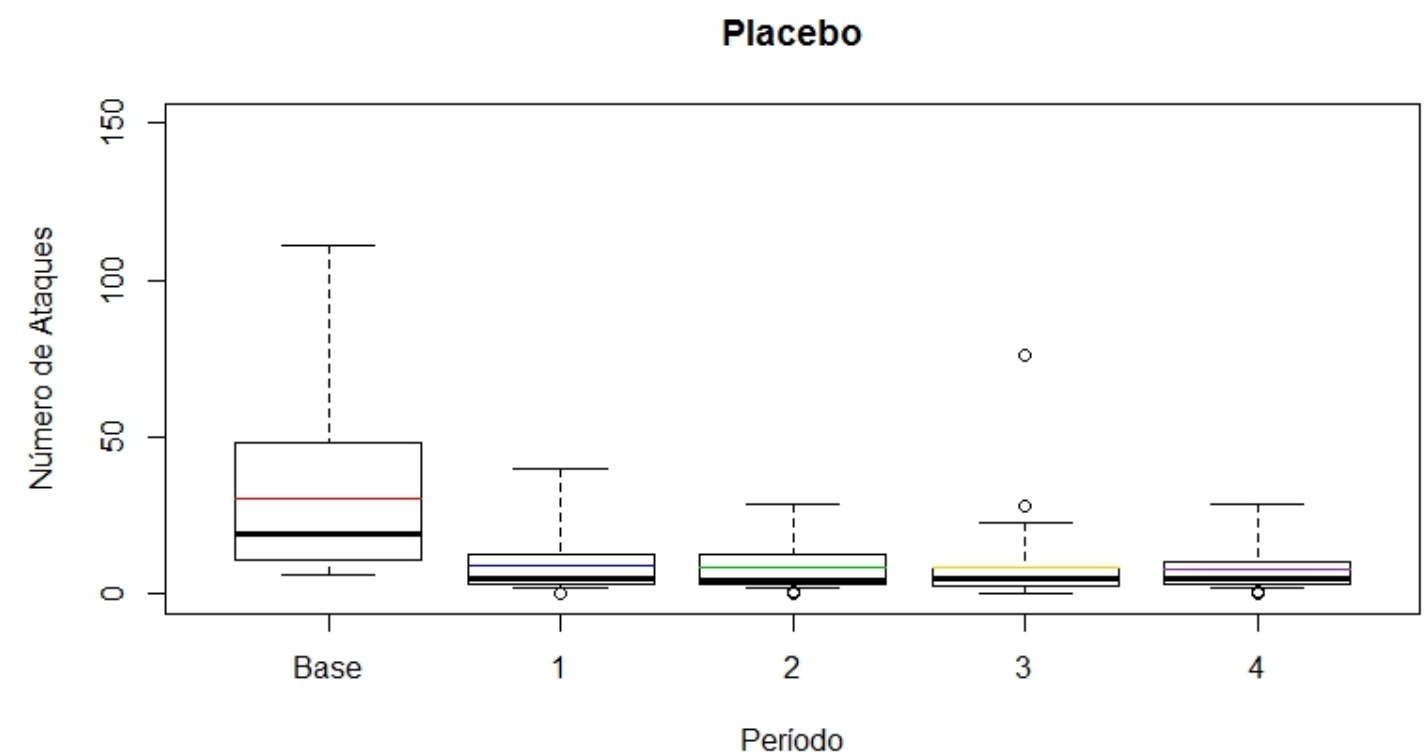

\section{Progabide}

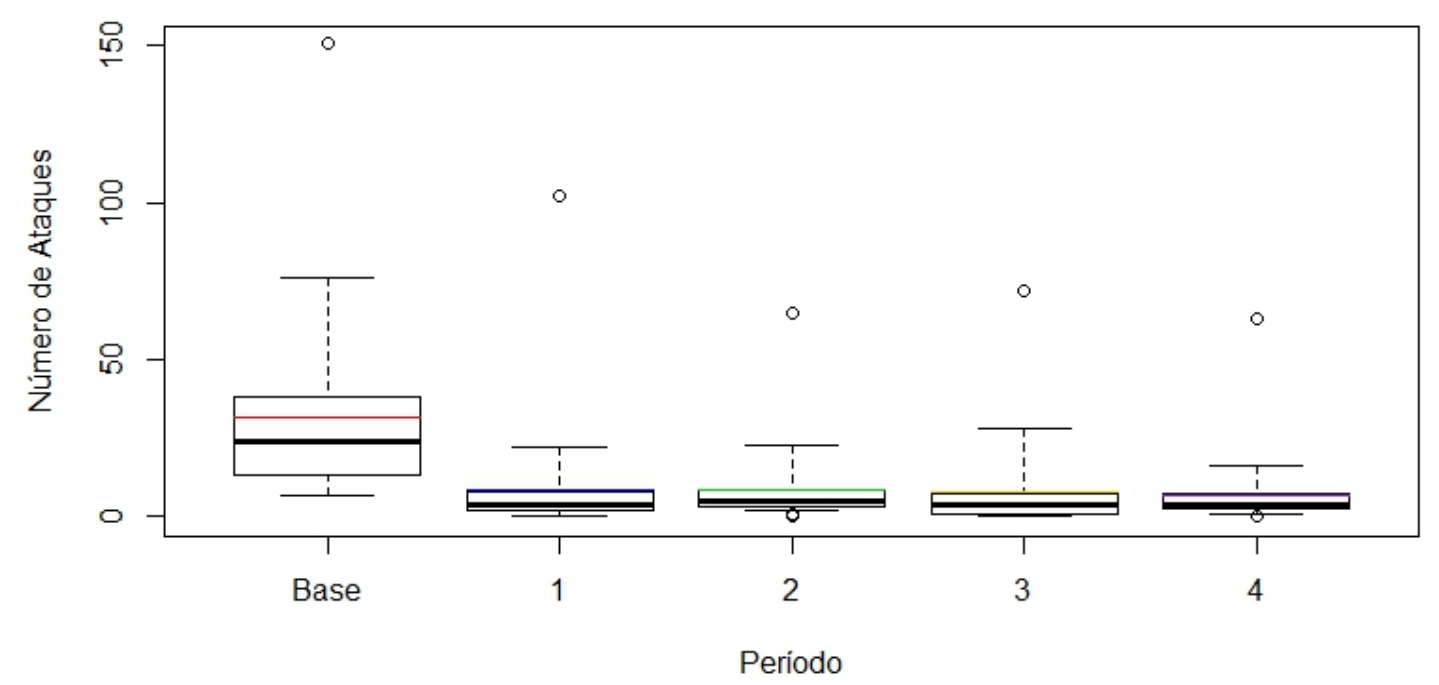

Figura 4.13: Boxplot robusto do número de ataques epilépticos segundo o tratamento e o período.

Esse banco de dados já foi analisado por diversos autores e há diferentes formas de abordar o problema.

O texto de Breslow (1996) faz uma revisão dos métodos usados após o ajuste de um MLG, e entre os problemas que são vistos estão a sobredispersão e a correlação. O banco de dados do exemplo no qual o texto é baseado é o mesmo dos pacientes epilépticos. Uma importante visão do texto é a de comparar o logaritmo da quantidade de ataques epilépticos através do tempo, identificando o paciente \#207 como um ponto aberrante. O texto utiliza um modelo com variáveis explicativas transformadas e ajusta a sobredispersão e a correlação através das abordagens de Jackknife, bootstrap e modelos com parâmetros na função de variância (binomial negativo).

Um modelo que leva em conta a diferença de tempo entre o período Base ( 8 semanas) e os períodos seguintes (2 semanas) é proposto no artigo de Zeger e Liang (1992), e também indica o paciente \#207 como um ponto aberrante. No entanto, os autores apenas mencionam que foi ajustado um modelo log-linear atrvés de EEGs. Para uma melhor comparação dos dados, os valores do período Base foram divididos por 4 . 
Fotouhi (2008) apresenta modelos multiníveis com diferentes componentes de erros aleatórios no preditor linear que são usados para analisar dados de contagem longitudinais de estudos clínicos. O método MCMC (Cadeia de Markov de Monte Carlo) é utilizado para a estimação, sendo considerados somente os valores dos momentos de 1 a 4 , ou seja, sem levar em conta os valores de Base.

No artigo de Thall e Vail (1990) são apresentadas equações de estimação para parâmetros de covariância. O modelo em questão leva em conta a sobredispersão, heteroscedasticidade e dependência entre observações repetidas. A abordagem é feita através de uma regressão de quase-verossimilhança similar à apresentada por Liang e Zeger (1986). O que é importante observar é que o artigo faz a modelagem retirando o paciente \#207 que aparenta ser um ponto aberrante.

Pelas conclusões apresentadas na literatura, podemos notar que diferentes autores tratam o modelo considerando a sobredispersão e a correlação entre as observações. Além disso, também utilizam meios para padronizar o tempo em semanas do acompanhamento e fazem a análise do modelo sem o paciente \#207.

Analisando o logaritmo da quantidade de ataque epilépticos através do tempo na Figura 4.14, notamos a discrepância do paciente \#207, que possui valores mais elevados do que os demais.

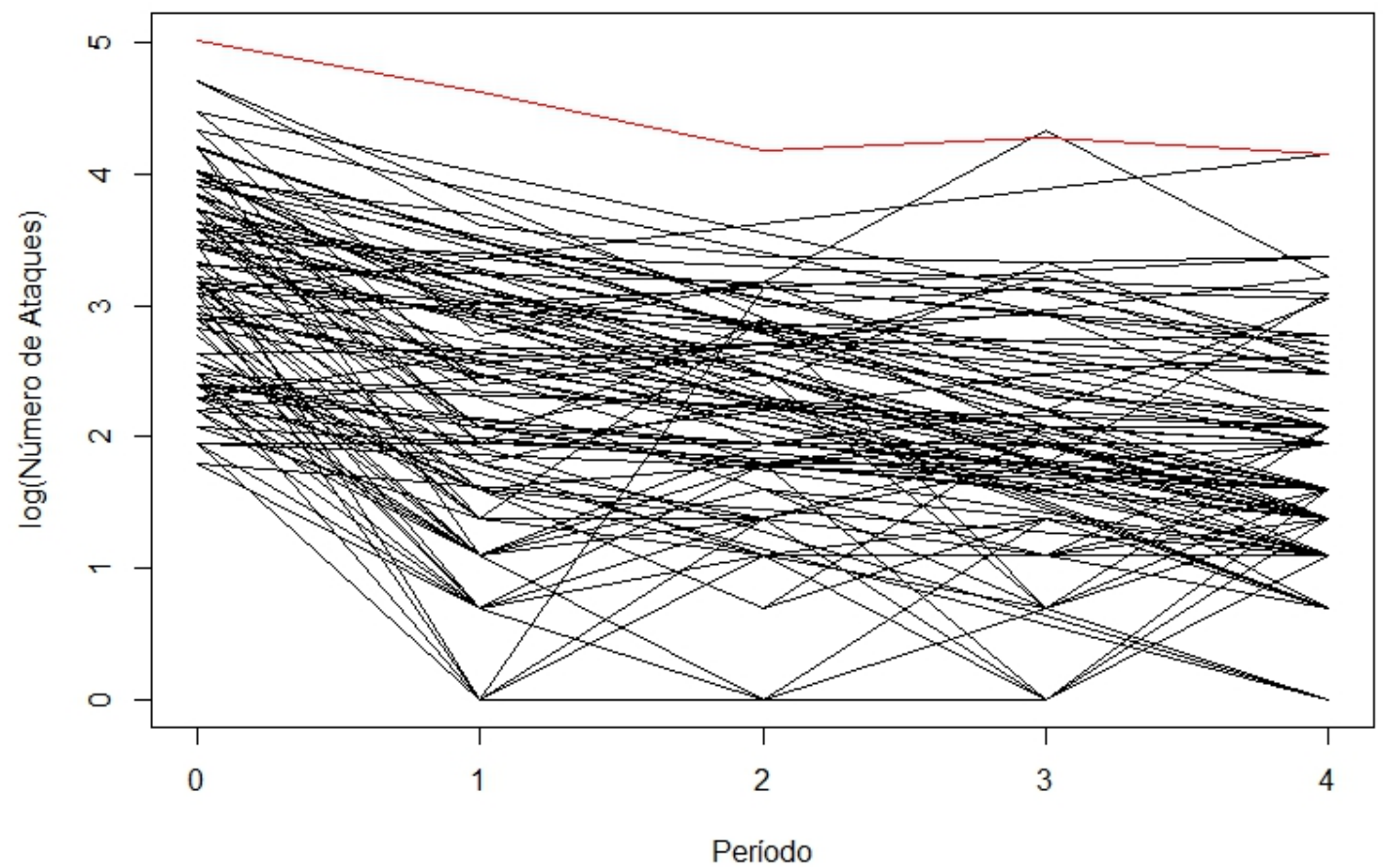

Figura 4.14: Gráfico de perfis do logaritmo do número de ataques epilépticos segundo o período.

Outra opção que foi apresentada na literatura é a de considerar somente os momentos de 2 semanas, ou seja, os períodos 1 a 4 . De fato, a interpretação fica mais fácil, como é possível observar pelo gráfico descrito na Figura 4.15. 


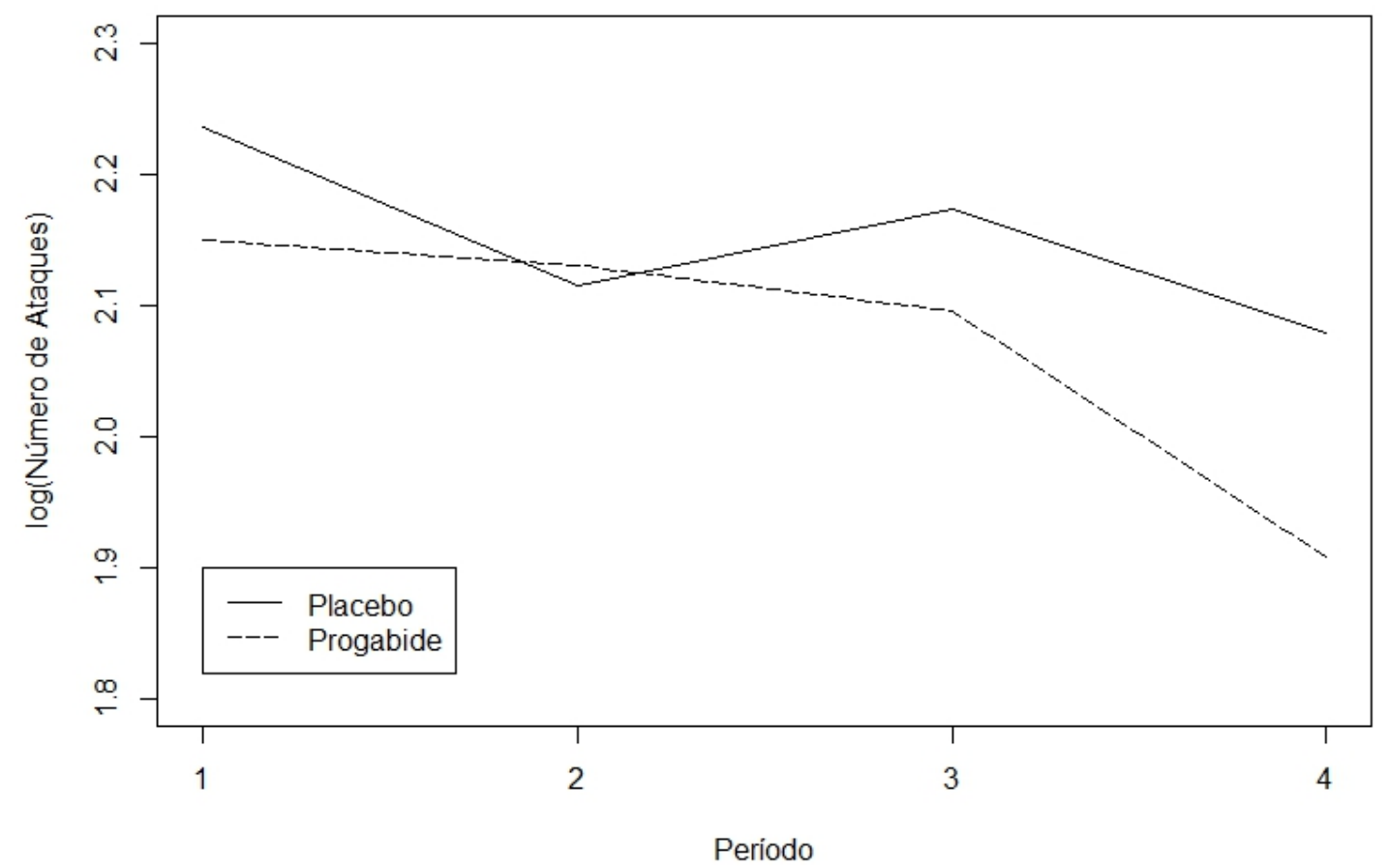

Figura 4.15: Gráfico da média do logaritmo do número de ataques epilépticos segundo o tratamento e o período.

Dsse gráfico podemos notar como o uso da droga Progabide parece ter diminuido a média do logaritmo da quantidade de ataques. A partir dessa proposta e das propostas apresentadas na literatura, vamos padronizar as semanas de cada período através da inclusão de um offset no modelo, e faremos a comparação dos modelos com e sem o paciente \#207.

\subsubsection{Selecionando o Modelo}

Para selecionar o melhor modelo vamos considerar primeiramente as seguintes variáveis:

- $y_{i j}$ : número de ataques epilépticos do paciente $i$ no momento $j$;

- Grupo ${ }_{i}$ : variável binária que indica se o $i$-ésimo paciente estava no grupo do tratamento (1) ou placebo (0);

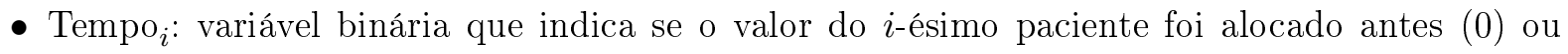
após (1) o começo do tratamento;

- $m_{j}$ : variável que leva em conta os diferentes períodos de observação, sendo 8 quando $j=0$ e 2 quando $j>0$;

para $i=1, \ldots, 59$ que indicam o paciente e $j=0,1,2,3,4$ que indica o momento em que foi observada a quantidade de ataques epilépticos.

Essas variáveis estão de acordo com o que foi apresentado em Zeger e Liang (1992). Para uma primeira análise, vamos verificar se um modelo linear normal se ajusta adequadamente aos dados. Então, temos o seguinte modelo:

$y_{i j}=\log \left(m_{j}\right)+\beta_{0}+\beta_{1} \mathrm{Grupo}_{i}+\beta_{2} \mathrm{Tempo}_{i}+\beta_{3} \mathrm{Grupo}_{i} \times \mathrm{Tempo}_{i}+\epsilon_{i j}, \quad i=1, \ldots, 59, \quad$ e $\quad j=0,1,2,3,4$, 
Tabela 4.19: Estimativa de parâmetros do modelo linear normal ajustado no $R$ aos dados de pacientes com epilepsia.

\begin{tabular}{c|r|r|r|r}
\hline Efeito & Estimativa & Erro Padrão & Valor-z & Valor-p \\
\hline Intercepto & 28,706 & 3,089 & 9,290 & $<0,001$ \\
GrupoT & 0,859 & 4,262 & 0,200 & 0,840 \\
TempoAp & $-20,792$ & 3,454 & $-6,020$ & $<0,001$ \\
GrupoT:TempoAp & $-1,499$ & 4,765 & $-0,310$ & 0,753 \\
\hline
\end{tabular}

com a suposição de que $\epsilon_{i j} \stackrel{\text { iid }}{\sim} \mathrm{N}\left(0, \sigma^{2}\right)$. Os parâmetros estimados são apresentados na Tabela 4.19.

Tivemos um $R^{2}$ ajustado no valor de 0,235 , que é relativamente baixo, o que era de esperar considerando que a variável resposta não é normal. Como a quantidade de ataques epilépticos é uma contagem um modelo que faz mais sentido ajustar é um MLG com resposta Poisson, seguindo as seguintes suposições:

- $y_{i j} \stackrel{i n d}{\sim} \operatorname{Poisson}\left(\mu_{i j}\right), i=1, \ldots, 59 \quad$ e $\quad j=0,1,2,3,4$;

- $\log \left(\mu_{i j}\right)=\log \left(m_{j}\right)+\beta_{0}+\beta_{1} \mathrm{Grupo}_{i}+\beta_{2} \mathrm{Tempo}_{i}+\beta_{3} \mathrm{Grupo}_{i} \times \mathrm{Tempo}_{i}$.

Os resultados que foram encontrados ao ajustar o modelo acima estão na Tabela 4.20.

Tabela 4.20: Estimativa dos parâmetros do modelo log-linear de Poisson ajustado no $R$ aos dados de pacientes com epilepsia.

\begin{tabular}{c|r|r|r|r}
\hline Efeito & Estimativa & Erro Padrão & Valor - z & Valor - p \\
\hline Intercepto & 1,348 & 0,034 & 39,570 & $<0,001$ \\
GrupoT & 0,028 & 0,047 & 0,590 & 0,555 \\
TempoAp & 0,112 & 0,047 & 2,390 & 0,017 \\
GrupoT:TempoAp & $-0,105$ & 0,065 & $-1,610$ & 0,107 \\
\hline
\end{tabular}

O AIC que foi encontrado para esse modelo foi de 4645 e o desvio de 3575, 4 (291 graus de liberdade). Faremos uma análise de diagnóstico para verificar se o modelo se ajusta bem aos dados, apresentada na Figura 4.16.
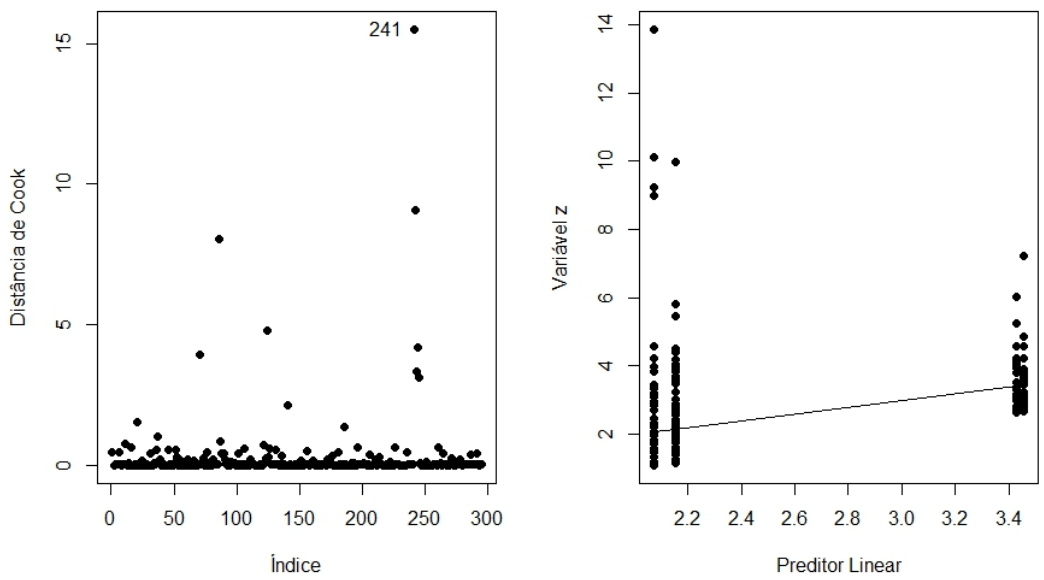

Figura 4.16: Gráficos do diagnóstico referentes ao ajuste no $R$ do modelo log-linear de Poisson aos dados de pacientes com epilepsia. 
Pela Figura 4.16 notamos que o ponto discrepante pela distância de Cook refere-se ao paciente \#207

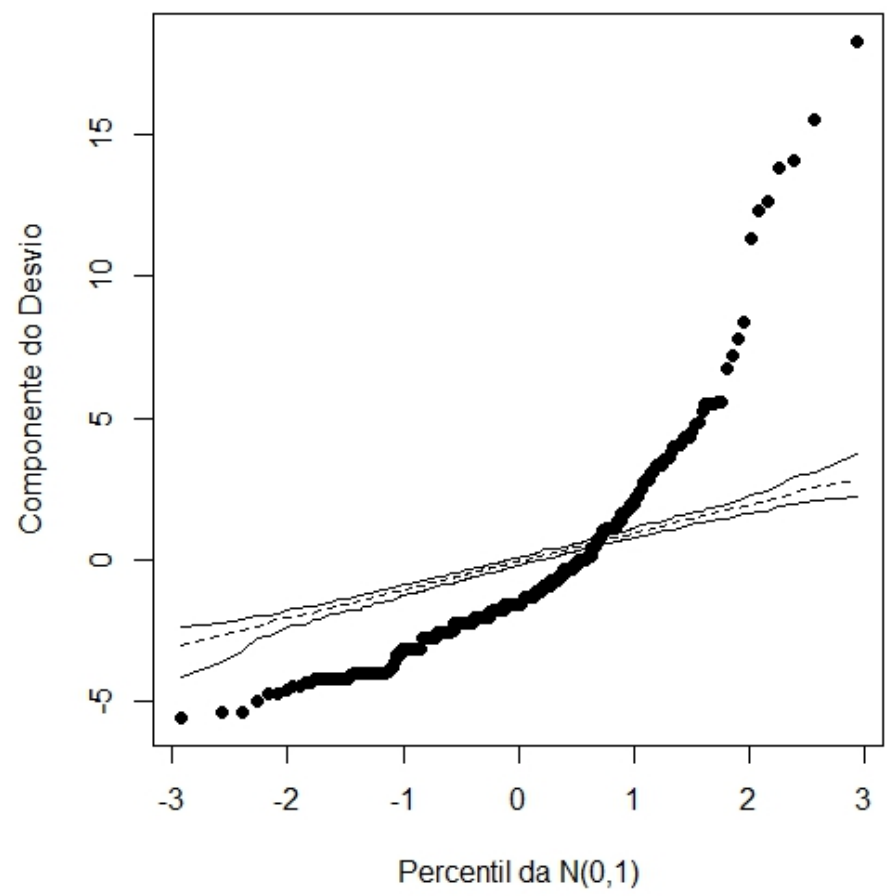

Figura 4.17: Gráfico normal de probabilidades referente ao ajuste no $R$ do modelo log-linear de Poisson aos dados de pacientes com epilepsia.

Pela Figura 4.17 notamos fortes indícios de sobredispersão no modelo com resposta Poisson, que pode ser confirmada ao relembrar a média e a varância dos dados na Tabela 4.15. Para contornar esse problema vamos ajustar um modelo com resposta binomial negativa. Teremos as seguintes suposições:

- $y_{i j} \stackrel{i n d}{\sim} \mathrm{BN}\left(\mu_{i j}, \nu\right), i=1, \ldots, 59 \quad$ e $\quad j=0,1,2,3,4$;

- $\log \left(\mu_{i j}\right)=\log \left(m_{j}\right)+\beta_{0}+\beta_{1} \mathrm{Grupo}_{i}+\beta_{2} \mathrm{Tempo}_{i}+\beta_{3} \mathrm{Grupo}_{i} \times \mathrm{Tempo}_{i}$.

Os resultados que foram encontrados a partir desse modelo estão descritos na Tabela 4.21.

Tabela 4.21: Estimativas dos parâmetros referentes ao ajuste no $R$ de um modelo log-linear $B N$ aos dados de pacientes com epilepsia.

\begin{tabular}{c|r|r|r|r}
\hline Efeito & Estimativa & Erro Padrão & Valor $-\mathrm{z}$ & Valor - p \\
\hline Intercepto & 1,348 & 0,188 & 7,190 & $<0,001$ \\
GrupoT & 0,028 & 0,259 & 0,110 & 0,915 \\
TempoAp & 0,112 & 0,211 & 0,530 & 0,597 \\
GrupoT:TempoAp & $-0,105$ & 0,292 & $-0,360$ & 0,720 \\
$\hat{\nu}$ & 1,051 & 0,093 & & \\
\hline
\end{tabular}

E o AIC encontrado para esse modelo foi de 2032 e o desvio de 334, 87 (291 graus de liberdade). Novamente faremos uma análise de diagnóstico. 

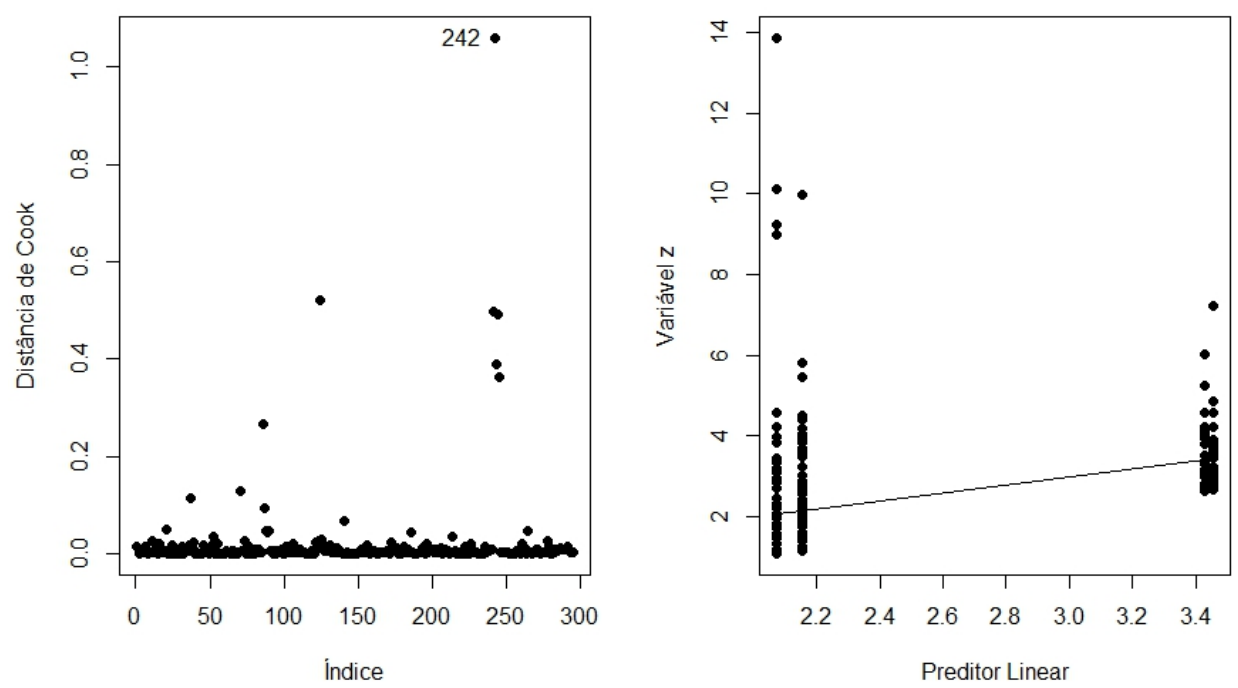

Figura 4.18: Gráficos do diagnóstico referentes ao ajuste no $R$ de um modelo log-linear BN aos dados de pacientes com epilepsia.

Agora, aparece a observação 242, do paciente \#207 como um ponto influente. E pelo gráfico normal de probabilidades descrito na Figura 4.19 nota-se uma melhora na qualidade do ajuste com relação ao modelo log-linear de Poisson.

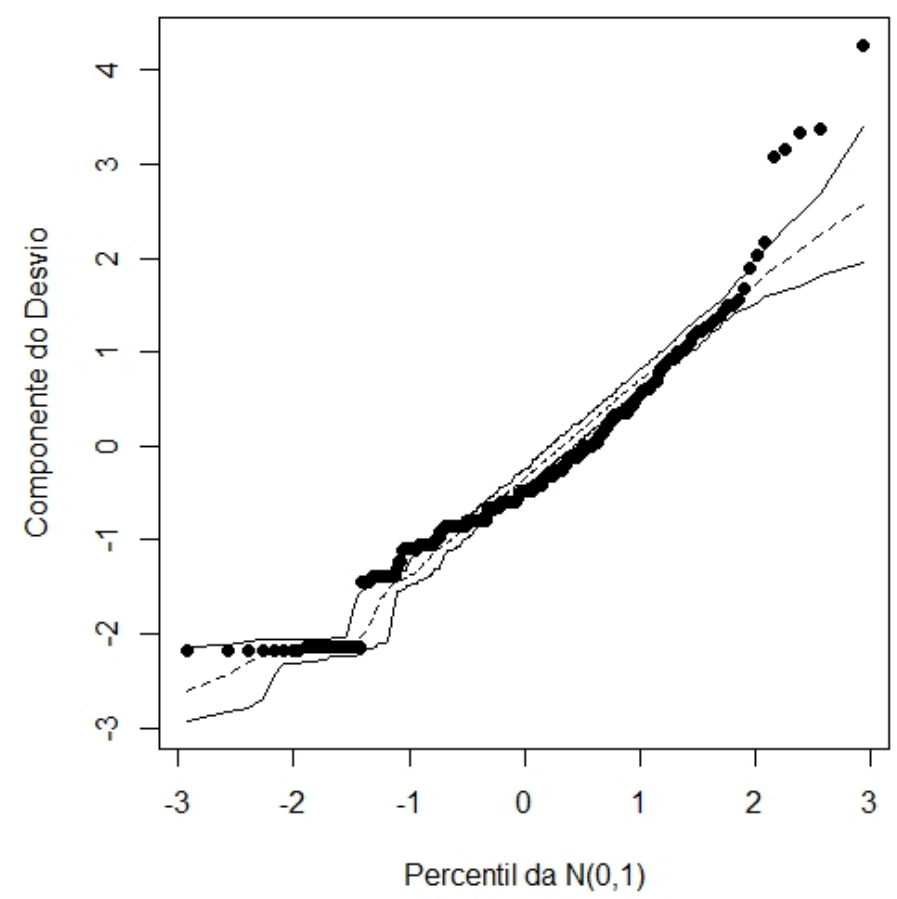

Figura 4.19: Gráfico normal de probabilidades referente ao ajuste no $R$ de um modelo log-linear $B N$ aos dados de pacientes com epilepsia. 
O que não está sendo levado em conta nos modelos propostos acima é a correlação que existe entre as observações de um mesmo paciente. Como é proposto nesta dissertação vamos ajustar modelos EEG-Poisson e EEG-BN e fazer as comparações.

O modelo EEG com resposta Poisson será ajustado levando em conta as seguintes suposições:

- $y_{i j} \sim \operatorname{Poisson}\left(\mu_{i j}\right), i=1, \ldots, 59 \quad$ e $\quad j=0,1,2,3,4$;

- $\log \left(\mu_{i j}\right)=\log \left(m_{j}\right)+\beta_{0}+\beta_{1} \mathrm{Grupo}_{i}+\beta_{2} \mathrm{Tempo}_{i}+\beta_{3} \mathrm{Grupo}_{i} \times \mathrm{Tempo}_{i}$

- $\operatorname{Corr}\left(y_{i j}, y_{i j^{\prime}}\right)=\rho$ para $j \neq j^{\prime}$ e $\operatorname{Corr}\left(y_{i j}, y_{i j^{\prime}}\right)=1$ para $j=j^{\prime}$.

Os resultados desse ajuste são apresentados na Tabela 4.22.

Tabela 4.22: Estimativas dos parâmetros do modelo EEG-Poisson ajustado no $R$ aos dados de pacientes com epilepsia.

\begin{tabular}{c|r|r|r|r}
\hline Efeito & Estimativa & Erro Padrão & Valor-z & Valor-p \\
\hline Intercepto & 1,348 & 0,157 & 8,560 & $<0,001$ \\
GrupoT & 0,028 & 0,222 & 0,141 & 0,900 \\
TempoAp & 0,112 & 0,116 & 0,964 & 0,330 \\
GrupoT:TempoAp & $-0,105$ & 0,213 & 0,490 & 0,620 \\
Escala & 19,400 & 8,690 &. &. \\
\hline
\end{tabular}

Com QIC encontrado através do pacote MuMIn de -8137 e a matriz trabalho estimada é apresentada na Tabela 4.23

Tabela 4.23: Matriz trabalho estimada referente ao modelo EEG-Poisson ajustado no $R$ aos dados de pacientes com epilepsia.

$$
\left[\begin{array}{ccccc}
1 & 0,777 & 0,777 & 0,777 & 0,777 \\
0,777 & 1 & 0,777 & 0,777 & 0,777 \\
0,777 & 0,777 & 1 & 0,777 & 0,777 \\
0,777 & 0,777 & 0,777 & 1 & 0,777 \\
0,777 & 0,777 & 0,777 & 0,777 & 1
\end{array}\right]
$$

Alguns gráficos de diagnóstico são apresentados na Figura 4.20.
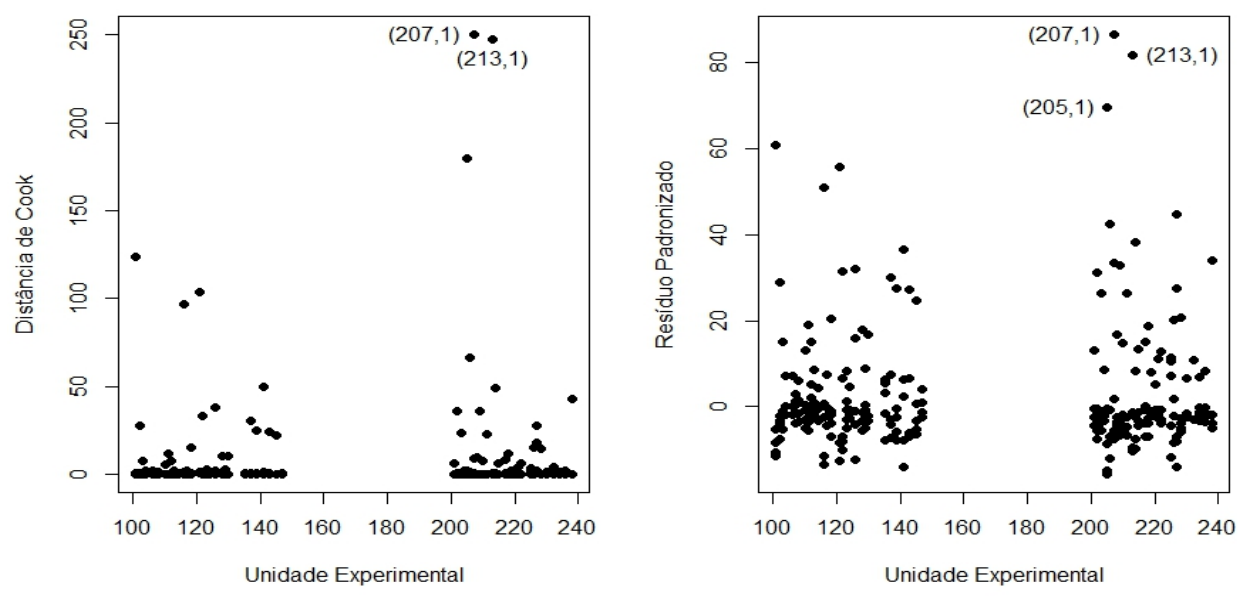

Figura 4.20: Gráficos de diagnóstico referentes ao ajuste do modelo EEG-Poisson no $R$ aos dados de pacientes com epilepsia. 


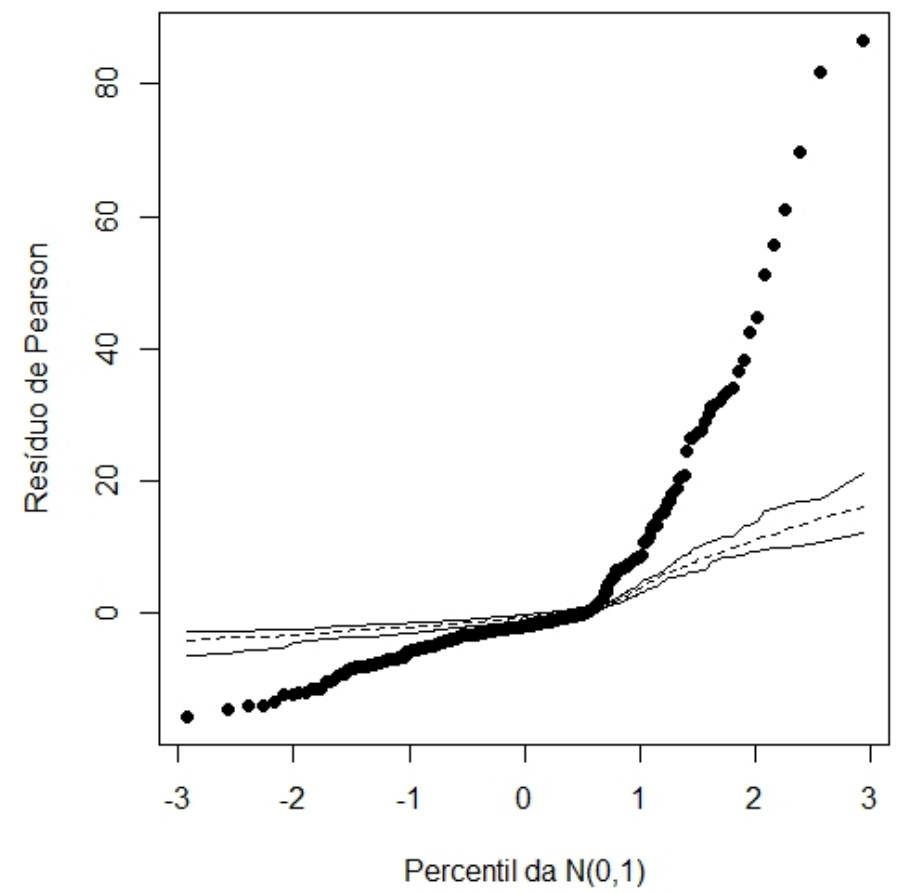

Figura 4.21: Gráfico normal de probabilidades referente ao ajuste do modelo EEG-Poisson no $R$ aos dados de pacientes com epilepsia.

Como já era de se esperar o modelo não se ajusta bem os dados e os pontos que são destacados nos gráficos de diagnóstico são do paciente \#207, \#205 e \#213. Além disso, vale a pena ressaltar pelos resultados encontrados na Tabela 4.22, que os efeitos não são significantes marginalmente. Como no $\mathrm{R}$ ainda não temos uma função que faça o EEGs com resposta binomial negativa, vamos encontrar o resultado no SAS. Para efeito de comparação vamos reestimar o EEG-Poisson também no SAS.

Tabela 4.24: Estimativas dos parâmetros do modelo EEG-Poisson ajustado no SAS aos dados de pacientes com epilepsia.

\begin{tabular}{c|r|r|r|r|r|r}
\hline Efeito & Estimativa & Erro Padrão & \multicolumn{2}{|c|}{ IC 95\% } & Valor-z & Valor-p \\
\hline Intercepto & 1,348 & 0,151 & 1,052 & 1,644 & 8,920 & $<0,001$ \\
GrupoT & 0,028 & 0,207 & $-0,378$ & 0,433 & 0,130 & 0,894 \\
TempoAp & 0,112 & 0,155 & $-0,191$ & 0,415 & 0,720 & 0,469 \\
GrupoT:TempoAp & $-0,105$ & 0,220 & $-0,535$ & 0,326 & $-0,480$ & 0,634 \\
Escala & 19,678 &. &. &. &. &. \\
\hline
\end{tabular}

O QIC é -661 e matriz trabalho estimada é apresentada na Tabela 4.25.

Tabela 4.25: Matriz trabalho estimada referente ao modelo EEG-Poisson ajustado no SAS aos dados de pacientes com epilepsia.

$$
\left[\begin{array}{ccccc}
1 & 0,771 & 0,771 & 0,771 & 0,771 \\
0,771 & 1 & 0,771 & 0,771 & 0,771 \\
0,771 & 0,771 & 1 & 0,771 & 0,771 \\
0,771 & 0,771 & 0,771 & 1 & 0,771 \\
0,771 & 0,771 & 0,771 & 0,771 & 1
\end{array}\right]
$$

Os valores estimados no modelo EEG-Poisson tanto no R quanto no SAS são praticamente 
iguais. Vamos ver então como fica o resultado para o modelo EEG com resposta binomial negativa levando em conta as seguintes suposições:

- $y_{i j} \sim \mathrm{BN}\left(\mu_{i j}, \nu\right), i=1, \ldots, 59 \quad$ e $\quad j=0,1,2,3,4$;

- $\log \left(\mu_{i j}\right)=\log \left(m_{j}\right)+\beta_{0}+\beta_{1} \mathrm{Grupo}_{i}+\beta_{2} \mathrm{Tempo}_{i}+\beta_{3} \mathrm{Grupo}_{i} \times \mathrm{Tempo}_{i}$;

- $\operatorname{Corr}\left(y_{i j}, y_{i j^{\prime}}\right)=\rho$ para $j \neq j^{\prime}$ e $\operatorname{Corr}\left(y_{i j}, y_{i j^{\prime}}\right)=1$ para $j=j^{\prime}$.

Os resultados desse ajuste são apresentados na Tabela 4.26.

Tabela 4.26: Estimativa dos parâmetros do modelo EEG-BN ajustado no SAS aos dados de pacientes com epilepsia.

\begin{tabular}{c|r|r|r|r|r|r}
\hline Efeito & Estimativa & Erro Padrão & \multicolumn{2}{|c|}{ IC 95\% } & Valor-z & Valor-p \\
\hline Intercepto & 1,348 & 0,256 & 0,845 & 1,850 & 5,260 & $<0,001$ \\
GrupoT & 0,028 & 0,354 & $-0,665$ & 0,720 & 0,080 & 0,938 \\
TempoAp & 0,112 & 0,145 & $-0,172$ & 0,396 & 0,770 & 0,440 \\
GrupoT:TempoAp & $-0,105$ & 0,200 & $-0,497$ & 0,287 & $-0,520$ & 0,601 \\
Escala & 1,869 &. &. &. &. &. \\
\hline
\end{tabular}

O QIC é - 8523 e a matriz de correlação estimada está descrita na Tabela 4.27. Não é apresentado no SAS a estimativa $\hat{\nu}$.

Tabela 4.27: Matriz trabalho estimada referente ao modelo EEG-BN ajustado no SAS aos dados de pacientes com epilepsia.

$$
\left[\begin{array}{ccccc}
1 & 0,750 & 0,750 & 0,750 & 0,750 \\
0,750 & 1 & 0,750 & 0,750 & 0,750 \\
0,750 & 0,750 & 1 & 0,750 & 0,750 \\
0,750 & 0,750 & 0,750 & 1 & 0,750 \\
0,750 & 0,750 & 0,750 & 0,750 & 1
\end{array}\right]
$$

De mesma forma como aconteceu com o modelo EEG-Poisson, os efeitos do modelo EEG-BN também não são significantes marginalmente. Uma característica das EEGs que devemos levar em conta, é a matriz de correlação que é definida nas suposições do modelo. Vamos ver quais os valores do QIC encontrados quando são utilizadas outras estruturas de correlação nas suposições do modelo EEG-BN.

- Simétrica $=-8523,277$

- Independente $=-8523,277$

- $\operatorname{AR}(1)=-8151,219$

- Não-estruturada $=-6768,955$.

Como podemos notar, a estrutura simétrica e a independente levaram ao mesmo QIC. Para esse estudo vamos escolher a estrutura simétrica.

\subsubsection{Melhorando o Modelo}

Através das técnicas de diagnóstico conseguimos perceber algumas observações que podem ser consideradas pontos influentes e aberrantes. As observações do paciente \#207 foram as mais aparentes nesse trabalho e também foram mencionadas na literatura. Por isso, vamos comparar o modelo completo e o modelo sem essas observações para verificar se existe uma melhora ao retirá-las. As análises foram feitas no SAS com as suposições do modelo EEG-BN considerando a matriz de trabalho simétrica. 
Tabela 4.28: Estimativas do modelo EEG-BN ajustado no SAS (com e sem o paciente \#207) aos dados de pacientes com epilepsia.

\begin{tabular}{c|r|r}
\hline Efeito & Modelo Completo & Modelo S/ Paciente \#207 \\
\hline Intercepto & $1,348(0,256)$ & $1,348(0,187)$ \\
GrupoT & $0,028(0,354)$ & $-0,107(0,261)$ \\
TermpoAp & $0,112(0,145)$ & $0,112(0,135)$ \\
GrupoT:TempoAp & $-0,105(0,200)$ & $-0,302(0,189)$ \\
\hline QIC & $-8523,277$ & $-10007,703$ \\
\hline
\end{tabular}

Apresentamos na Tabela 4.29 os resultados do ajuste do modelo EEG-BN aos dados de pacientes com epilepsia sem o paciente \#207. Como podemos notar nenhum efeito é marginalmente significativo. Na Tabela 4.30 a matriz trabalho estimada é apresentada.

Tabela 4.29: Estimativas dos parâmetros do modelo EEG-BN ajustado no SAS aos dados de pacientes com epilepsia, sem o paciente \#20\%.

\begin{tabular}{c|r|r|r|r|r|r}
\hline Efeito & Estimativa & Erro Padrão & \multicolumn{2}{|c|}{ IC 95\% } & Valor-z & Valor-p \\
\hline Intercepto & 1,348 & 0,187 & 0,981 & 1,715 & 7,200 & $<0,001$ \\
GrupoT & $-0,107$ & 0,261 & $-0,618$ & 0,404 & $-0,410$ & 0,682 \\
TempoAp & 0,112 & 0,135 & $-0,154$ & 0,377 & 0,830 & 0,409 \\
GrupoT:TempoAp & $-0,302$ & 0,189 & $-0,674$ & 0,069 & $-1,600$ & 0,110 \\
Escala & 1,279 & &. &. &. &. \\
\hline
\end{tabular}

Tabela 4.30: Matriz trabalho estimada referente ao modelo EEG-BN ajustado no SAS aos dados de pacientes com epilepsia, sem o paciente \#20\%.

$$
\left[\begin{array}{ccccc}
1 & 0,591 & 0,591 & 0,591 & 0,591 \\
0,591 & 1 & 0,591 & 0,591 & 0,591 \\
0,591 & 0,591 & 1 & 0,591 & 0,591 \\
0,591 & 0,591 & 0,591 & 1 & 0,591 \\
0,591 & 0,591 & 0,591 & 0,591 & 1
\end{array}\right]
$$

A seguir fazemos uma comparação de vários submodelos segundo o QIC.

- $\log \left(\mu_{i j}\right)=\log \left(m_{j}\right)+\beta_{0}+\beta_{1} T_{i}+\beta_{2}$ Tempo $_{j}+\beta_{3} T_{i}$ Tempo $_{j}$

$\mathrm{QIC}=-10007,703 ;$

- $\log \left(\mu_{i j}\right)=\log \left(m_{j}\right)+\beta_{0}+\beta_{1} T_{i}+\beta_{2} \mathrm{Tempo}_{j}$

$\mathrm{QIC}=-9985,337$

- $\log \left(\mu_{i j}\right)=\log \left(m_{j}\right)+\beta_{0}+\beta_{1} T_{i}$

$\mathrm{QIC}=-10178,819$

- $\log \left(\mu_{i j}\right)=\log \left(m_{j}\right)+\beta_{0}+\beta_{2}$ Tempo $_{j}$

$\mathrm{QIC}=-9230,102 ;$

- $\log \left(\mu_{i j}\right)=\log \left(m_{j}\right)$

$\mathrm{QIC}=-9347,213 ;$

Tabela 4.31: Estimativas dos parâmetros do modelo EEG-BN ajustado no SAS aos dados de pacientes com epilepsia sem o paciente \#207 e com menor QIC.

\begin{tabular}{c|r|r|r|r|r|r}
\hline Efeito & Estimativa & Erro Padrão & \multicolumn{2}{|c|}{ IC 95\% } & Valor-z & Valor-p \\
\hline Intercepto & 1,429 & 0,159 & 1,118 & 1,740 & 9,010 & $<0,001$ \\
GrupoT & $-0,315$ & 0,223 & $-0,752$ & 0,122 & $-1,410$ & 0,157 \\
Escala & 1,259 &. &. &. &. &. \\
\hline
\end{tabular}

Matriz trabalho estimada descrita na Tabela 4.32. 
Tabela 4.32: Matriz trabalho estimada referente ao modelo EEG-BN ajustado no SAS aos dados de pacientes com epilepsia, sem o paciente \#207 e com menor QIC.

$$
\left[\begin{array}{ccccc}
1 & 0,588 & 0,588 & 0,588 & 0,588 \\
0,588 & 1 & 0,588 & 0,588 & 0,588 \\
0,588 & 0,588 & 1 & 0,588 & 0,588 \\
0,588 & 0,588 & 0,588 & 1 & 0,588 \\
0,588 & 0,588 & 0,588 & 0,588 & 1
\end{array}\right]
$$

Como podemos notar o modelo EEG-BN com o menor QIC e sem o paciente \#207 tem apenas como significativo o intercepto. Logo, não foi possível detectar ao nível de $10 \%$ nenhum efeito no tratamento de pacientes com epilepsia.

\subsubsection{Método Alternativo}

Vamos a seguir ajustar esses dados através de cópulas Gaussianas com resposta Poisson. Os resultados são descritos na Tabela 4.33 .

Tabela 4.33: Estimativas dos parâmetros do modelo de cópulas Gaussianas com resposta Poisson ajustado no $R$ aos dados de pacientes com epilepsia.

\begin{tabular}{c|r|r|r|r}
\hline Efeito & Estimativa & Erro Padrão & Valor-z & Valor-p \\
\hline Intercepto & 1,149 & 0,037 & 31,141 & $<0,001$ \\
GrupoT & 0,004 & 0,051 & 0,072 & 0,943 \\
TempoAp & 0,118 & 0,042 & 2,809 & 0,005 \\
GrupoT:TempoAp & $-0,303$ & 0,061 & $-4,984$ & $<0,001$ \\
\hline
\end{tabular}

O AIC do modelo acima foi de 2689, 800. Na Tabela 4.34 apresentamos os resultados do ajuste de cópulas Gaussianas com resposta BN.

Tabela 4.34: Estimativas dos parâmetros do modelo de cópulas Gaussianas com resposta BN ajustado no $R$ aos dados de pacientes com epilepsia.

\begin{tabular}{c|r|r|r|r}
\hline Efeito & Estimativa & Erro Padrão & Valor-z & Valor-p \\
\hline Intercepto & 1,398 & 0,166 & 8,428 & $<0,001$ \\
GrupoT & 0,085 & 0,219 & 0,387 & 0,699 \\
TempoAp & 0,081 & 0,112 & 0,726 & 0,468 \\
GrupoT:TempoAp & $-0,201$ & 0,156 & $-1,292$ & 0,197 \\
$\nu$ & 0,810 & 0,118 & 6,845 & $<0,001$ \\
\hline
\end{tabular}

O AIC do modelo acima foi de 1827, 100. Ao testarmos outras estruturas de correlação foi visto que a simétrica apresentou o menor AIC. Também foi ajustado o modelo com resposta binomial negativa e sem o paciente \#207, cujos resultados são descritos na Tabela 4.35.

Tabela 4.35: Estimativa dos parâmetros do modelo de cópulas Gaussianas com resposta BN ajustado no $R$ aos dados de pacientes com epilepsia, sem o paciente \#207.

\begin{tabular}{c|r|r|r|r}
\hline Efeito & Estimativa & Erro Padrão & Valor-z & Valor-p \\
\hline Intercepto & 1,378 & 0,150 & 9,177 & $<0,001$ \\
GrupoT & $-0,074$ & 0,202 & $-0,367$ & 0,714 \\
TempoAp & 0,084 & 0,111 & 0,756 & 0,450 \\
GrupoT:TempoAp & $-0,334$ & 0,156 & $-2,138$ & 0,033 \\
$\nu$ & 0,637 & 0,093 & 6,848 & $<0,001$ \\
\hline
\end{tabular}

O AIC do modelo acima foi de 1749,600. Apresentamos a seguir o valor do AIC para todos os 
submodelos referentes ao ajuste de cópulas Gaussianas com resposta BN aos dados de pacientes com epilepsia e sem o paciente \#207.

- $\log \left(\mu_{i j}\right)=\log \left(m_{j}\right)+\beta_{0}+\beta_{1} T_{i}+\beta_{2}$ Tempo $_{j}+\beta_{3} T_{i}$ Tempo $_{j}$

$$
\begin{aligned}
& \mathrm{AIC}=1749,600 ; \\
& \mathrm{AIC}=1752,100 ; \\
& \mathrm{AIC}=1751,400 ; \\
& \mathrm{AIC}=1753,800 ; \\
& \mathrm{AIC}=1752,600 ;
\end{aligned}
$$$$
\text { - } \log \left(\mu_{i j}\right)=\log \left(m_{j}\right)+\beta_{0}+\beta_{1} T_{i}+\beta_{2} \mathrm{Tempo}_{j}
$$$$
\text { - } \log \left(\mu_{i j}\right)=\log \left(m_{j}\right)+\beta_{0}+\beta_{1} T_{i}
$$$$
\text { - } \log \left(\mu_{i j}\right)=\log \left(m_{j}\right)+\beta_{0}+\beta_{2} \text { Tempo }_{j}
$$$$
\text { - } \log \left(\mu_{i j}\right)=\log \left(m_{j}\right)
$$

Portanto, através do ajuste do modelo de cópulas Gaussianas com resposta BN aos dados de pacientes com epilepsia e sem o paciente \#207 detectamos efeito de interação entre o Tratamento e o Tempo. Como a estimativa é negativa, concluímos que há efeito de tratamento. Esse resultado vai ao encontro de resultados obtidos por outros autores. Contudo, quando ajustamos modelos EEG-Poisson e EEG-BN não detectamos nenhum efeito, mesmo quando o paciente \#207 não é considerado. Notamos também que as estimativas obtidas nas EEGs pelo SAS e pelo R são similares. 


\subsection{Pacientes do German Health Reform}

Os dados sobre pacientes do German Health Reform são mais difíceis de analisar, por terem um grande número de variáveis, e um grande número de observações que estão desbalanceadas, ou seja, cada paciente possui um número de observações que varia de 1 a 5 , não sendo iguaL para todos.

\subsubsection{Análise Preliminar dos Dados}

Vamos ver como se comportam esses dados. Começamos pela variável número de visitas ao médico, cujas medidas resumo são descritas na Tabela 4.36.

Tabela 4.36: Medidas resumo do número de visitas ao médico.

\begin{tabular}{r|r}
\hline Média & Variância \\
\hline 3,176 & 34,247 \\
\hline
\end{tabular}

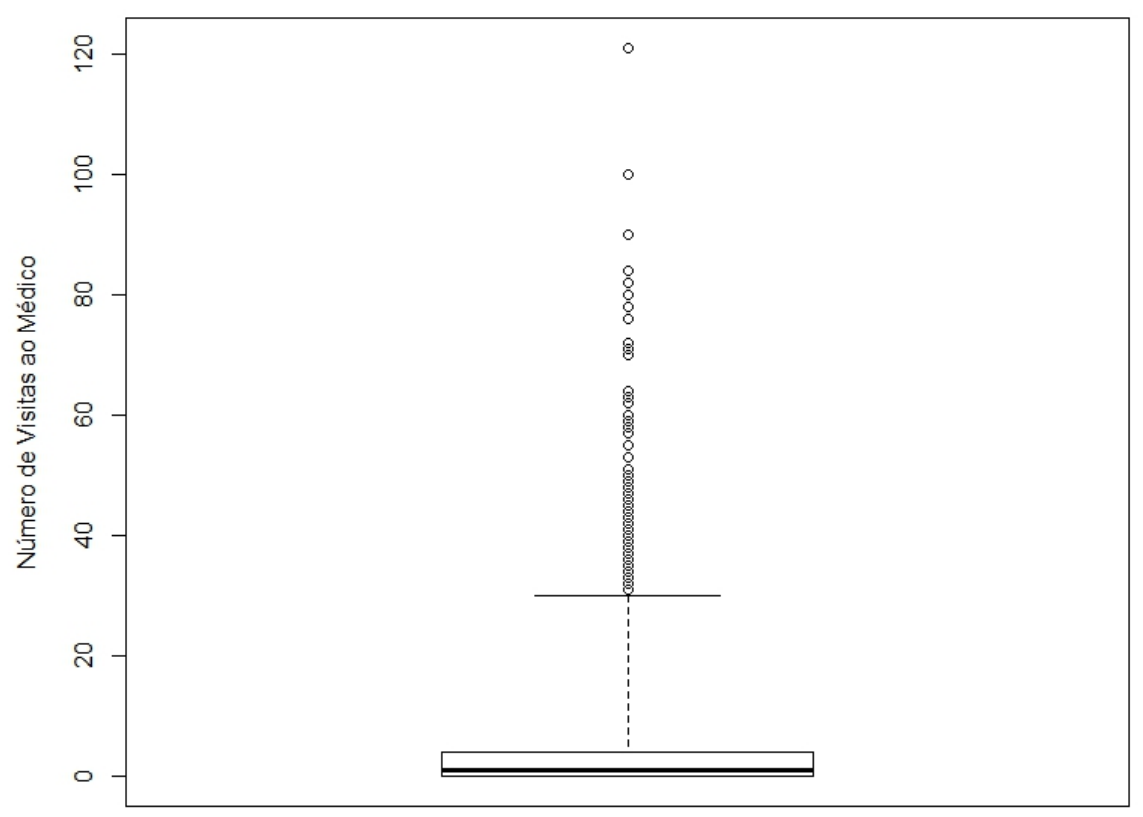

Figura 4.22: Boxplot robusto do número de visitas ao médico.

Pela Tabela 4.36 vemos como a variância é muito superior ao valor da média, já indicando uma sobredispersão para um modelo de Poisson. Além disso, pelo boxplot robusto notamos uma grande quantidade de outliers.

Vamos verificar agora o comportamento da variável número de visitas ao hospital segundo o número de visitas ao médico. 


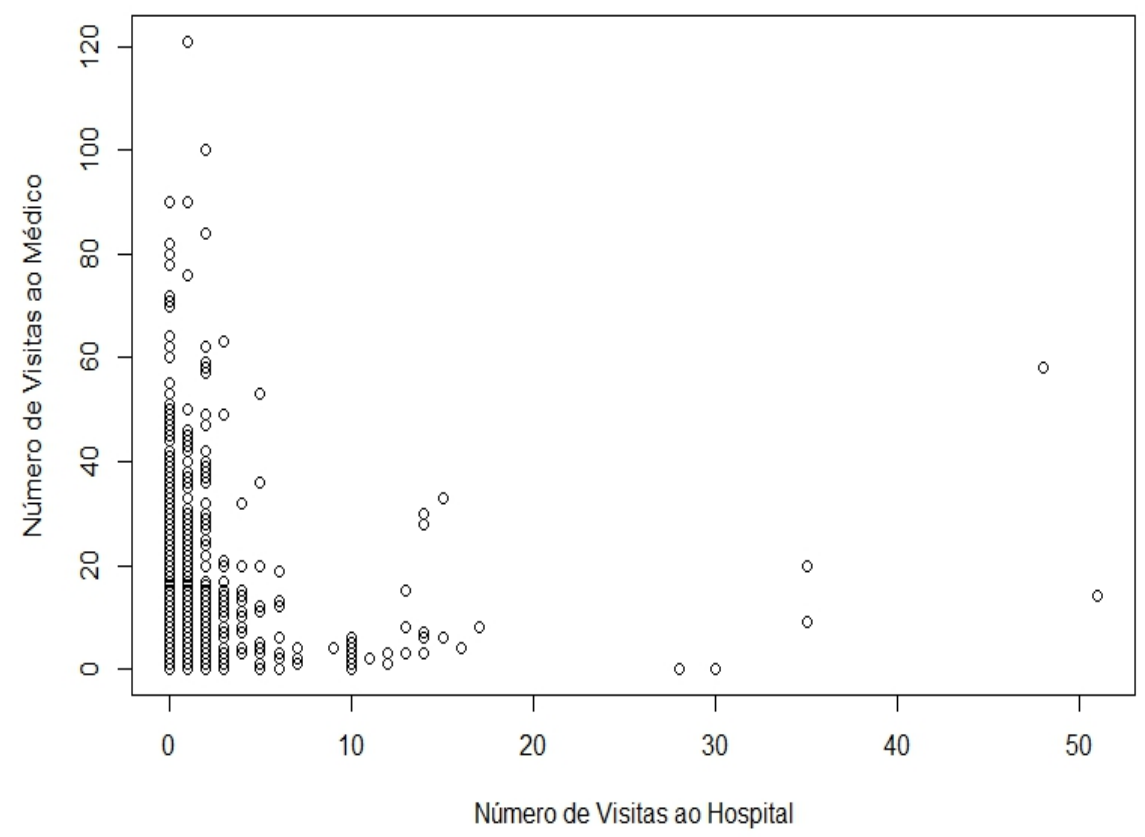

Figura 4.23: Gráfico de dispersão entre o número de visitas ao médico e o número de visitas ao hospital.

Pela Figura 4.23 notamos que à medida que aumenta o número de visitas que um paciente faz ao hospital diminui o número de visitas ao médico. Através dos anos notamos pela Tabela 4.37 que a média e a variância são parecidas nos 4 primeiros anos, sendo o ano 1988 um diferencial. E o gráfico boxplot na Figura 4.24, só mostra melhor essa condição.

Tabela 4.37: Medidas resumo do número de visitas ao médico segundo o ano.

\begin{tabular}{r|r|r}
\hline Ano & Média & Variância \\
\hline 1984 & 3,163 & 39,388 \\
1985 & 3,085 & 32,099 \\
1986 & 3,512 & 39,082 \\
1987 & 3,310 & 35,336 \\
1988 & 2,872 & 26,464 \\
\hline
\end{tabular}




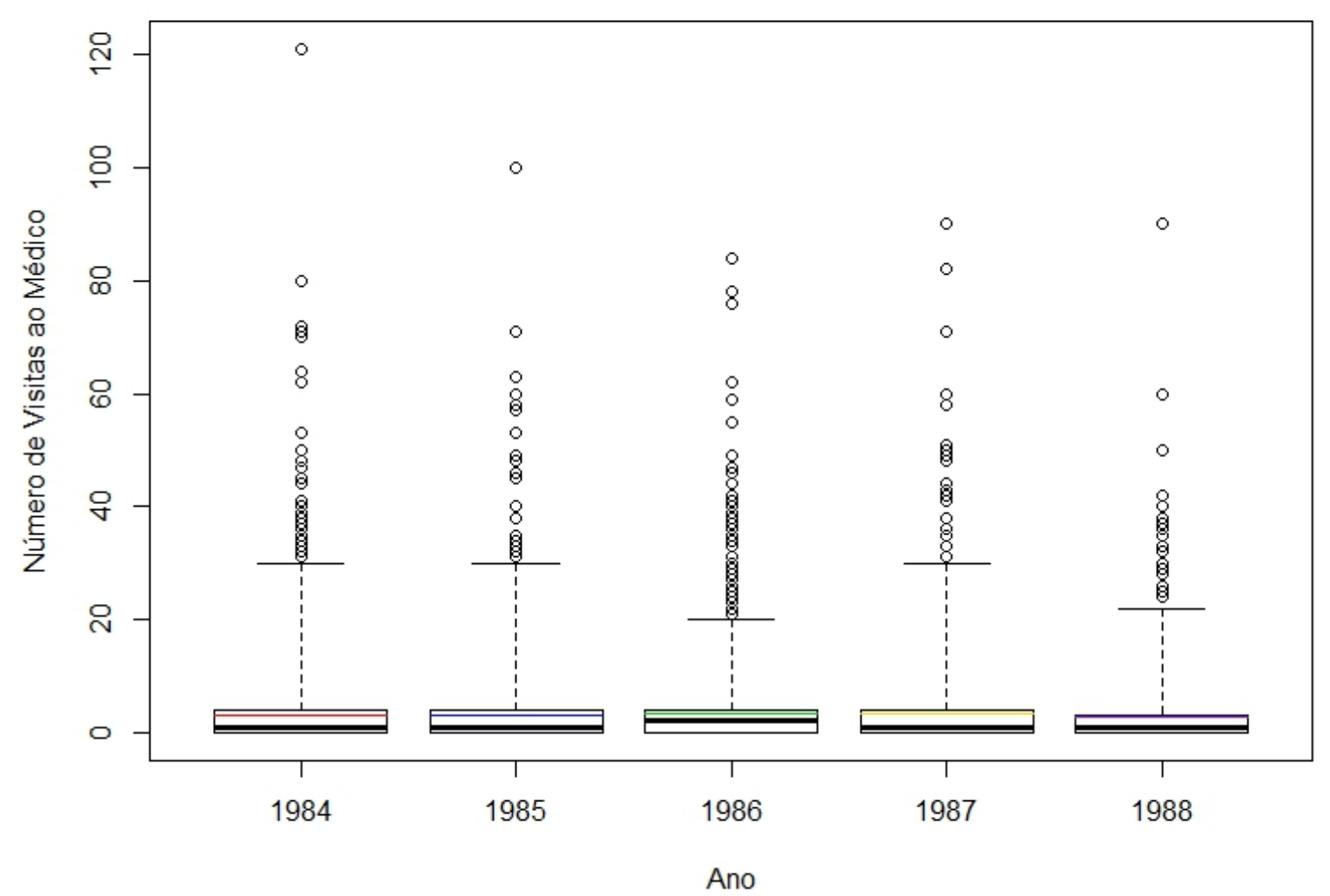

Figura 4.24: Boxplot robusto do número de visitas ao médico segundo o ano.

Comparando o número de visitas ao médico pela idade do paciente, conforme descrito na Figura 4.25 , não observamos nenhuma tendência.

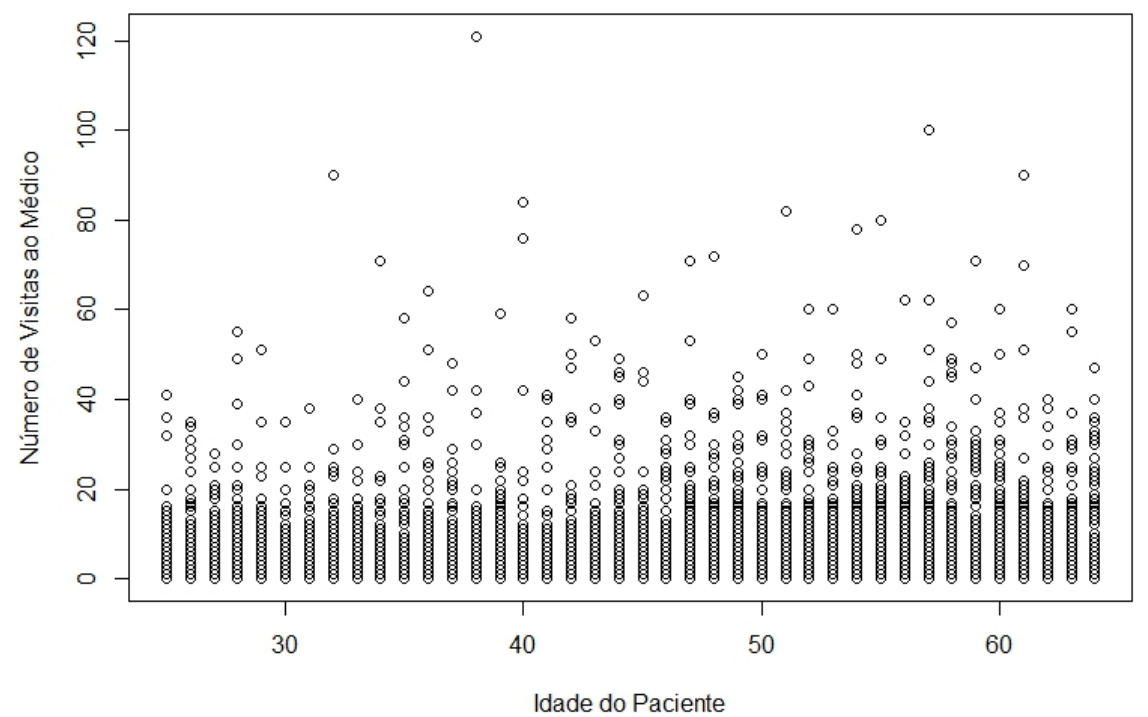

Figura 4.25: Gráfico de dispersão entre o número de visitas ao médico e a idade do paciente. 
O número médio de visitas ao médico quando os pacientes estavam trabalhando é bem maior do que quando não estavam trabalhando. O mesmo comportamento vale para a variância, como é descrito na Tabela 4.38 .

Tabela 4.38: Medidas resumo do número de visitas ao médico segundo a condição de trabalho.

\begin{tabular}{c|r|r}
\hline Estava trabalhando? & Média & Variância \\
\hline Não & 2,666 & 25,479 \\
Sim & 4,150 & 49,534 \\
\hline
\end{tabular}

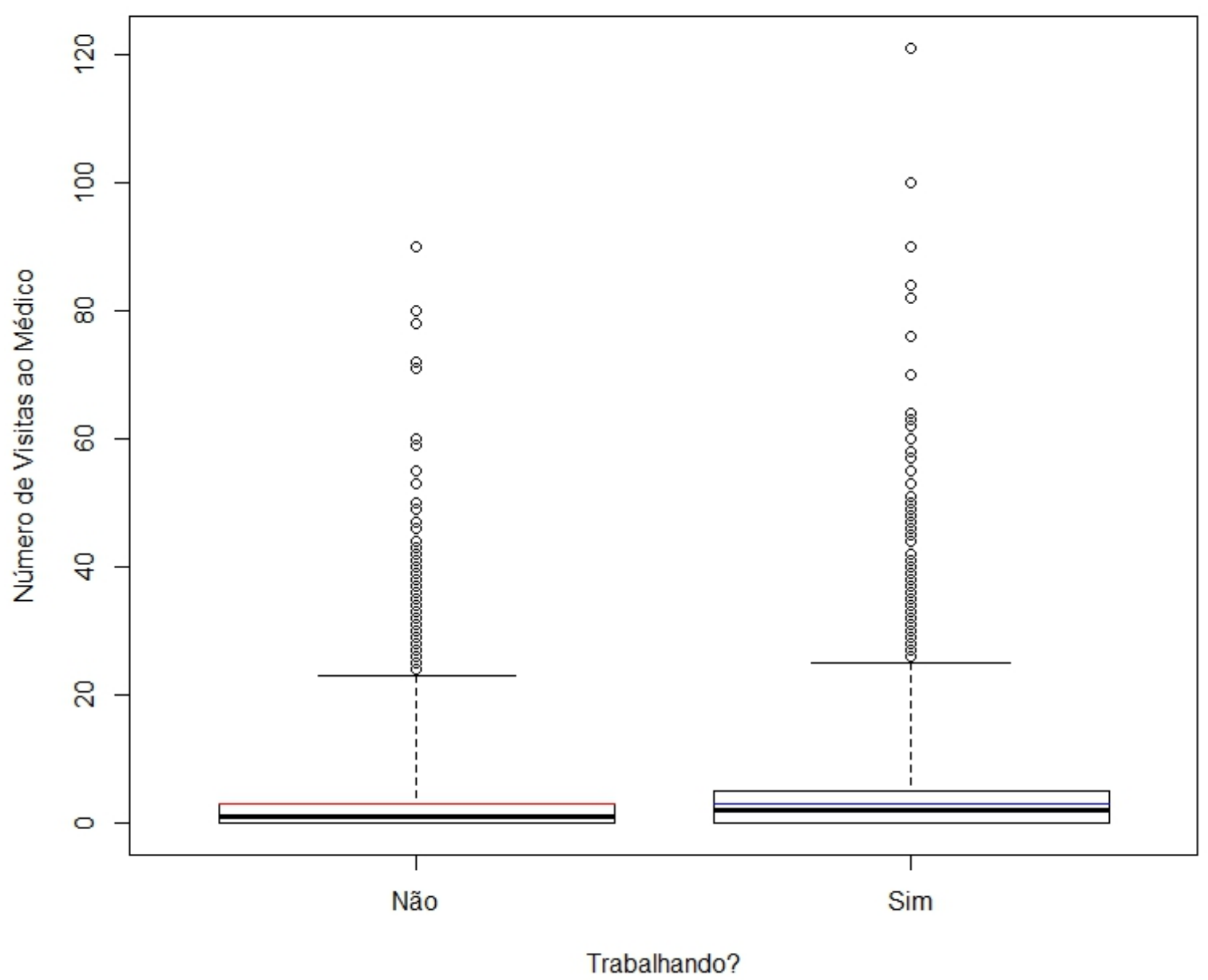

Figura 4.26: Boxplot robusto do número de visitas ao médico segundo a condição de trabalho. 
Vamos ver o comportamentodo número de visitas ao médico segundo o gênero, conforme descrito na Tabela 4.39.

Tabela 4.39: Medidas resumo do número de visitas ao médico segundo o gênero.

\begin{tabular}{c|r|r}
\hline Gênero & Média & Variância \\
\hline Masculino & 2,642 & 28,754 \\
Feminino & 3,754 & 39,547 \\
\hline
\end{tabular}

A Tabela 4.39 e a Figura 4.27 mostram que as visitas ao médico são em geral maiores entre as mulheres.

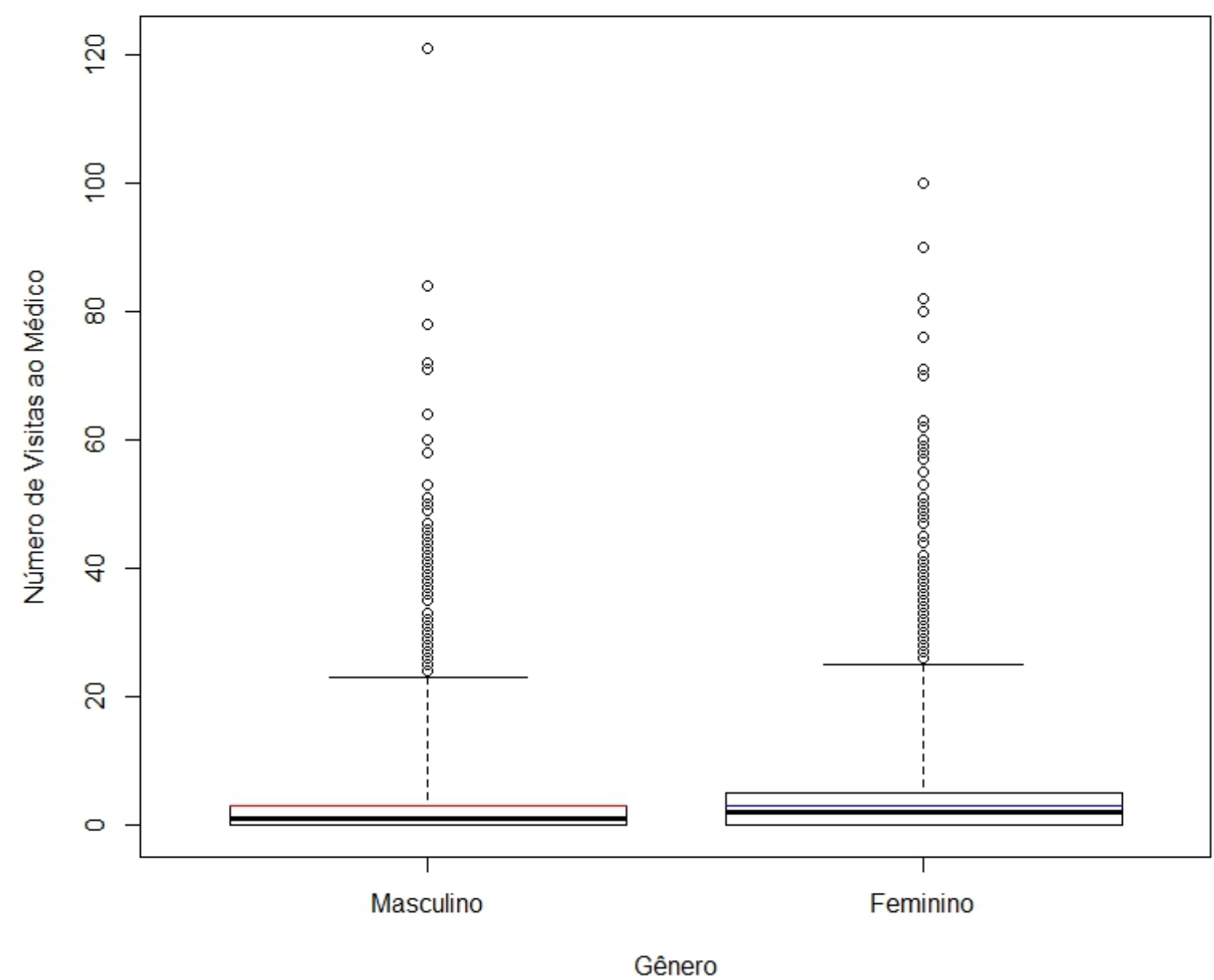

Figura 4.27: Boxplot robusto do número de visitas ao médico segundo o gênero. 
Analisando o número de visitas ao médico segundo o nível da educação, notamos fortes indícios de que quanto maior o nível educacional, menor o número de idas ao médico.

Tabela 4.40: Medidas resumo do número de visitas ao médico segundo o nível educaciional.

\begin{tabular}{c|r|r}
\hline Nível de Educação & Média & Variância \\
\hline 1 & 3,389 & 38,822 \\
2 & 2,925 & 20,528 \\
3 & 2,375 & 16,019 \\
4 & 1,929 & 13,286 \\
\hline
\end{tabular}

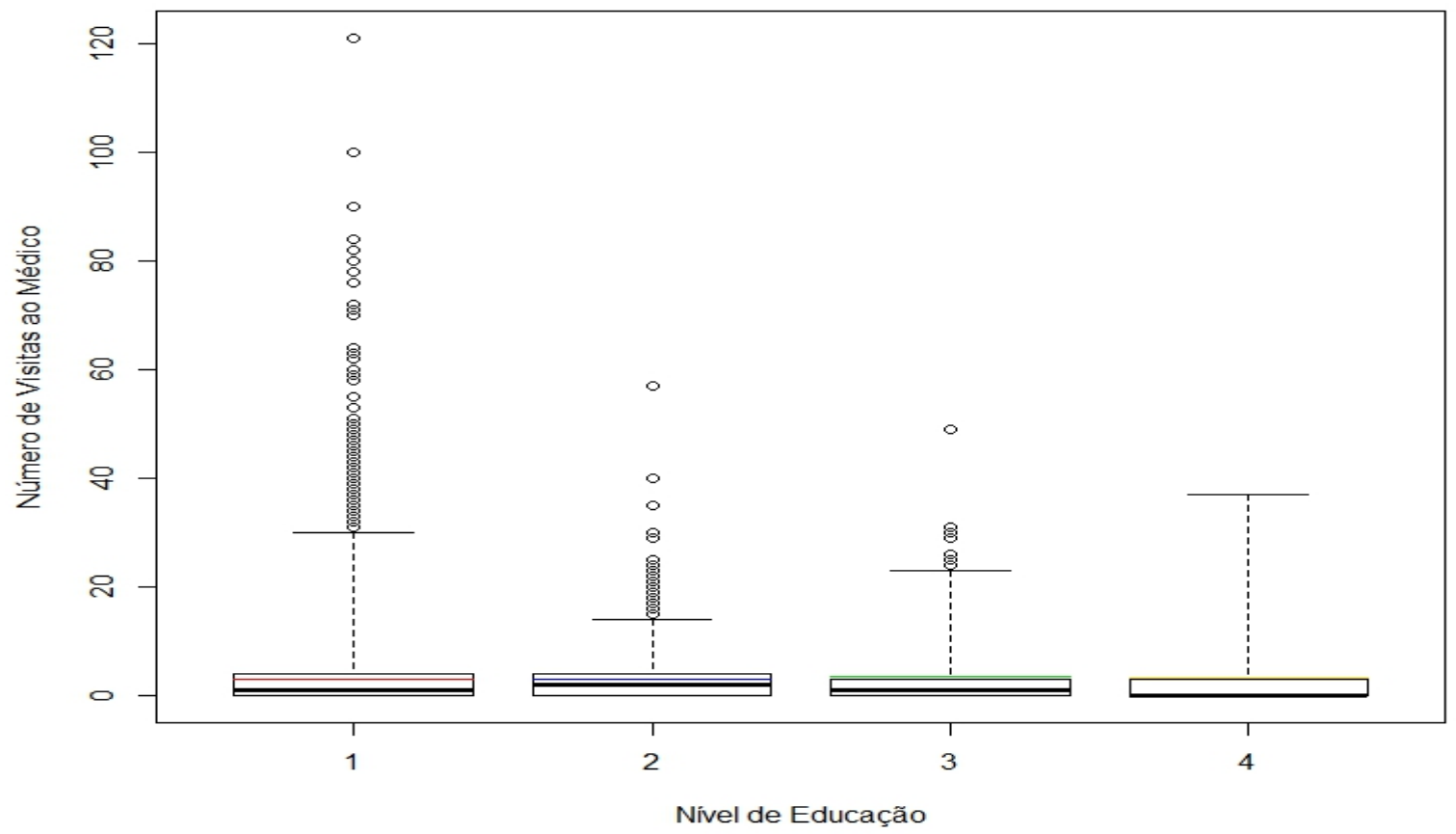

Figura 4.28: Boxplot robusto do número de visitas ao médico segundo o nível de educação. 
Vamos agora descrever o comportamento do número de vivitas ao médico segundo o estado civil (casado ou não casado).

Tabela 4.41: Medidas resumo do número de visitas ao médico segundo o estado civil.

\begin{tabular}{c|r|r}
\hline Casado? & Média & Variância \\
\hline Não & 3,207 & 38,932 \\
Sim & 3,167 & 32,878 \\
\hline
\end{tabular}

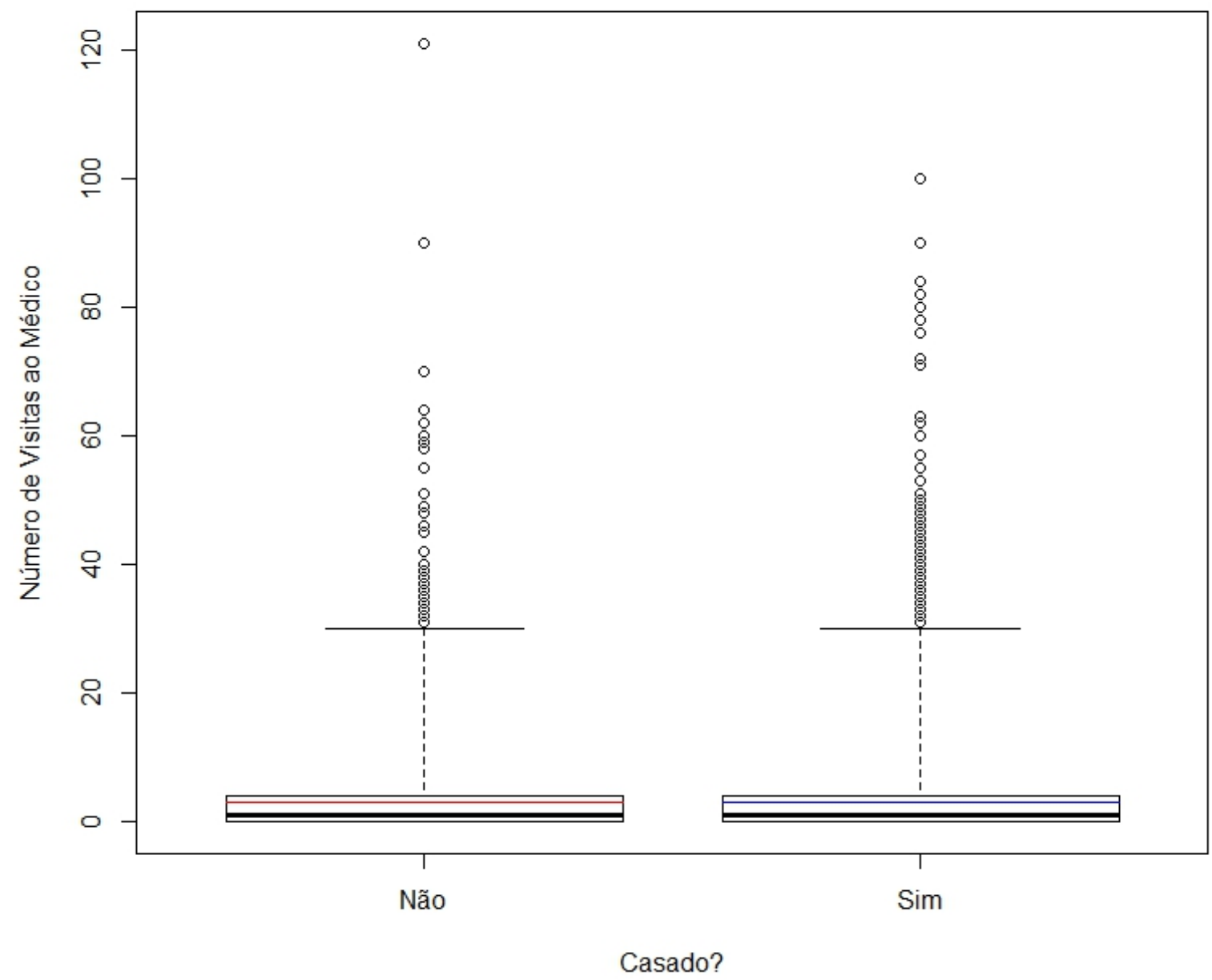

Figura 4.29: Boxplot robusto do número de visitas ao médico segundo o estado civil.

Não é possível perceber diferenças importantes entre a distirbuição do número de visitas ao médico nos grupos casado e não casado. 
Pela Tabela 4.42 e Figura 4.30 notamos que o número de visitas ao médico fica em geral menor para pacientes com filhos.

Tabela 4.42: Medidas resumo do número de visitas ao médico segundo a presença de filhos.

\begin{tabular}{c|r|r}
\hline Filhos? & Média & Variância \\
\hline Não & 3,616 & 40,780 \\
Sim & 2,551 & 24,310 \\
\hline
\end{tabular}

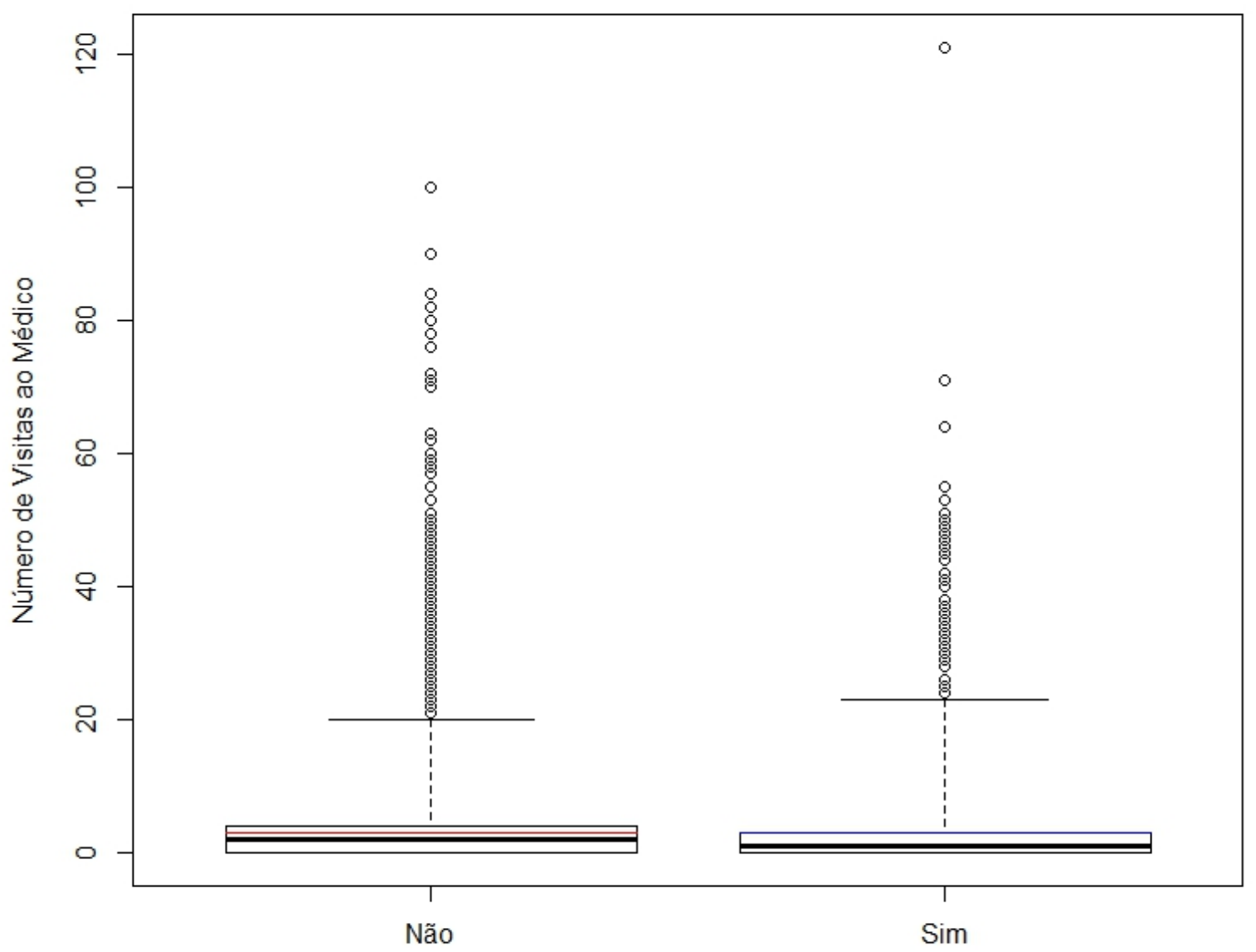

Filhos?

Figura 4.30: Boxplot robusto do número de visitas ao médico segundo a presença de filhos.

Esse banco de dados está descrito, em particular, nos livros de Hilbe (2014,2011).

Em Hilbe (2014) a análise dos dados por meio de modelos para dados de contagem é restrita ao ano de 1984. Já em Hilbe (2011) há aplicação do modelo EEG-BN para estudar o número de visitas ao médico, porém apenas com as variáveis explicativas gênero e nível educacional. 


\subsubsection{Selecionando o Modelo}

Para selecionar um submodelo adequado vamos considerar as seguintes variáveis:

- $y_{i j}$ : número de visitas ao médico do paciente $i$ no ano $j$;

- $H_{i j}$ : número de visitas ao hospital do paciente $i$ no ano $j$;

- $I_{i j}$ : idade do paciente $i$ no ano $j$ (em anos) ;

- $T_{i j}$ : variável binária se o $i$-ésimo paciente estava trabalhando no ano $j$ (1: sim, 0: não);

- $S_{i j}$ : variável binária se o $i$-ésimo paciente é do sexo feminino no ano $j$ (1: sim, 0: não);

- $N E 2_{i j}$ : variável binária se o nível educacional do paciente $i$ no ano $j$ é o 2 (1: sim, 0: não);

- $N E 3_{i j}$ : variável binária se o nível educacional do paciente $i$ no ano $j$ é o 3 (1: sim, 0: não);

- $N E 4_{i j}$ : variável binária se o nível educacional do paciente $i$ no ano $j$ é o 4 (1: sim, 0: não);

- $C_{i j}$ : variável binária se o $i$-ésimo paciente estava casado no ano $j$ (1: sim, 0: não);

- $F_{i j}$ : variável binária se o $i$-ésimo tinha filhos no ano $j$ (1: sim, 0: não).

O primeiro modelo que podemos ajustar é o modelo linear normal que será definido da seguinte maneira:

$$
y_{i j}=\beta_{0}+\beta_{1} H_{i j}+\beta_{2} I_{i j}+\beta_{3} T_{i j}+\beta_{4} S_{i j}+\beta_{5} N E 2_{i j}+\beta_{6} N E 3_{i j}+\beta_{7} N E 4_{i j}+\beta_{8} C_{i j}+\beta_{9} F_{i j}+\epsilon_{i j},
$$

para $i=1, \ldots, 6127$ e $j=1984, \ldots, 1988$, e a suposição de que $\epsilon_{i j} \stackrel{i i d}{\sim} \mathrm{N}\left(0, \sigma^{2}\right)$. As estimativas dos parâmetros são descritos na Tabela 4.43 .

Tabela 4.43: Estimativas dos parâmetros do modelo linear normal ajustado no $R$ aos dados de pacientes do German Health Reform.

\begin{tabular}{c|r|r|r|r}
\hline Efeito & Estimativa & Erro Padrão & Valor-z & Valor-p \\
\hline Intercepto & 0,693 & 0,205 & 3,383 & 0,001 \\
NVHospital & 0,839 & 0,044 & 19,210 & $<0,001$ \\
Idade & 0,052 & 0,004 & 12,387 & $<0,001$ \\
Trabalhando & 0,828 & 0,098 & 8,489 & $<0,001$ \\
Feminino & 0,584 & 0,091 & 6,434 & $<0,001$ \\
NE2 & $-0,240$ & 0,175 & $-1,374$ & 0,169 \\
NE3 & $-0,667$ & 0,147 & $-4,534$ & $<0,001$ \\
NE44 & $-0,896$ & 0,167 & $-5,365$ & $<0,001$ \\
Casado & $-0,181$ & 0,106 & $-1,711$ & 0,087 \\
Filhos & $-0,533$ & 0,095 & $-5,585$ & $<0,001$ \\
\hline
\end{tabular}

Tivemos um $R^{2}$ ajustado no valor de 0,054 , que é relativamente baixo, o que era de esperar considerando que a variável resposta não é normal. Como a variável dependente é uma contagem o ideal é ajustar um modelo com resposta Poisson, seguindo as seguintes suposições:

- $y_{i j} \stackrel{i n d}{\sim} \operatorname{Poisson}\left(\mu_{i j}\right), \quad i=1, \ldots, 6127 \quad$ e $\quad j=1984, \ldots, 1988$

- $\log \left(\mu_{i j}\right)=\beta_{0}+\beta_{1} H_{i j}+\beta_{2} I_{i j}+\beta_{3} T_{i j}+\beta_{4} S_{i j}+\beta_{5} N E 2_{i j}+\beta_{6} N E 3_{i j}+\beta_{7} N E 4_{i j}+\beta_{8} C_{i j}+\beta_{9} F_{i j}$.

Os resultados do ajuste no $\mathrm{R}$ estão descritos na Tabela 4.44. 
Tabela 4.44: Estimativas dos parâmetros do modelo log-linear de Poisson ajustado no $R$ aos dados de pacientes do German Health Reform.

\begin{tabular}{c|r|r|r|r}
\hline Efeito & Estimativa & Erro Padrão & Valor-z & Valor-p \\
\hline Intercepto & 0,369 & 0,022 & 16,907 & $<0,001$ \\
NVHospital & 0,067 & 0,001 & 51,435 & $<0,001$ \\
Idade & 0,016 & $<0,001$ & 37,335 & $<0,001$ \\
Trabalhando & 0,226 & 0,009 & 24,221 & $<0,001$ \\
Feminino & 0,197 & 0,009 & 21,806 & $<0,001$ \\
NE2 & $-0,069$ & 0,018 & $-3,871$ & $<0,001$ \\
NE3 & $-0,234$ & 0,016 & $-14,224$ & $<0,001$ \\
NE44 & $-0,377$ & 0,021 & $-18,193$ & $<0,001$ \\
Casado & $-0,046$ & 0,010 & $-4,619$ & $<0,001$ \\
Filhos & $-0,170$ & 0,010 & $-17,153$ & $<0,001$ \\
\hline
\end{tabular}

O AIC encontrado com o ajuste do modelo foi de 150822. O desvio do modelo foi de 113535 para 19599 graus de liberdade, e como podemos notar o desvio é muito superior indicando a possível existência de sobredispersão. Não foi possível construir os gráficos de diganóstico no R, em virtude da alta dimensão da matriz. Então, vamos ajustar um modelo com resposta binomial negativa com a seguinte estrutura:

- $y_{i j} \sim \mathrm{BN}\left(\mu_{i j}, \nu\right), \quad i=1, \ldots, 6127 \quad$ e $\quad j=1984, \ldots, 1988$

- $\log \left(\mu_{i j}\right)=\beta_{0}+\beta_{1} H_{i j}+\beta_{2} I_{i j}+\beta_{3} T_{i j}+\beta_{4} S_{i j}+\beta_{5} N E 2_{i j}+\beta_{6} N E 3_{i j}+\beta_{7} N E 4_{i j}+\beta_{8} C_{i j}+\beta_{9} F_{i j}$.

O algoritmo usando a função glm.nb no software $\mathrm{R}$ não convergiu de primeira, mas ao colocarmos um número máximo de 50 iterações retornou as estimativas descritas na Tabela 4.45 .

Tabela 4.45: Estimativas dos parâmetros do modelo log-linear binomial negativo ajustado no $R$ aos dados de pacientes do German Health Reform.

\begin{tabular}{c|r|r|r|r}
\hline Efeito & Estimativa & Erro Padrão & Valor-z & Valor-p \\
\hline Intercepto & 0,221 & 0,056 & 3,984 & $<0,001$ \\
NVHospital & 0,399 & 0,011 & 36,815 & $<0,001$ \\
Idade & 0,017 & 0,001 & 14,784 & $<0,001$ \\
Trabalhando & 0,191 & 0,026 & 7,379 & $<0,001$ \\
Feminino & 0,256 & 0,024 & 10,549 & $<0,001$ \\
NE2 & $-0,081$ & 0,047 & $-1,733$ & 0,083 \\
NE3 & $-0,207$ & 0,040 & $-5,147$ & $<0,001$ \\
NE44 & $-0,379$ & 0,047 & $-8,143$ & $<0,001$ \\
Casado & $-0,023$ & 0,028 & $-0,799$ & 0,424 \\
Filhos & $-0,158$ & 0,026 & $-6,152$ & $<0,001$ \\
$\hat{\nu}$ & 0,508 & 0,007 & & \\
\hline
\end{tabular}

O AIC obtido foi de 85237 e o desvio foi de 20242 com 19599 graus de liberdade, uma redução substancial com relação ao modelo log-linear de Poisson. Também não foi possível construir os gráficos de diagnóstico no R. Como sabemos que as observações de um mesmo paciente são correlacionadas, vamos ajustar agora modelos EEGs, começando com a resposta Poisson. Temos então as seguintes suposições:

- $y_{i j} \sim \operatorname{Poisson}\left(\mu_{i j}\right), \quad i=1, \ldots, 6127 \quad$ e $\quad j=1984, \ldots, 1988$

- $\log \left(\mu_{i j}\right)=\beta_{0}+\beta_{1} H_{i j}+\beta_{2} I_{i j}+\beta_{3} T_{i j}+\beta_{4} S_{i j}+\beta_{5} N E 2_{i j}+\beta_{6} N E 3_{i j}+\beta_{7} N E 4_{i j}+\beta_{8} C_{i j}+\beta_{9} F_{i j}$;

- $\operatorname{Corr}\left(y_{i j}, y_{i j^{\prime}}\right)=\rho$ para $j \neq j^{\prime}$ e $\operatorname{Corr}\left(y_{i j}, y_{i j^{\prime}}\right)=1$ para $j=j^{\prime}$. 
Fazendo o ajuste no SAS obtemos os resultados descritos na Tabela 4.46.

Tabela 4.46: Estimativas dos parâmetros do modelo EGG-Poisson ajustado no SAS aos dados de pacientes do German Health Reform.

\begin{tabular}{c|r|r|r|r|r|r}
\hline Efeito & Estimativa & Erro Padrão & \multicolumn{2}{|c|}{ IC 95\% } & Valor-z & Valor-p \\
\hline Intercepto & 0,348 & 0,084 & 0,183 & 0,513 & 4,130 & $<0,001$ \\
NVHospital & 0,061 & 0,004 & 0,053 & 0,070 & 13,980 & $<0,001$ \\
Idade & 0,017 & 0,002 & 0,014 & 0,020 & 10,220 & $<0,001$ \\
Trabalhando & 0,183 & 0,033 & 0,118 & 0,249 & 5,510 & $<0,001$ \\
Feminino & 0,213 & 0,037 & 0,141 & 0,284 & 5,840 & $<0,001$ \\
NE2 & $-0,056$ & 0,072 & $-0,197$ & 0,084 & $-0,790$ & 0,431 \\
NE3 & $-0,228$ & 0,066 & $-0,357$ & $-0,099$ & $-3,460$ & 0,001 \\
NE4 & $-0,332$ & 0,082 & $-0,493$ & $-0,171$ & $-4,050$ & $<0,001$ \\
Casado & $-0,076$ & 0,038 & $-0,151$ & $-0,001$ & $-1,990$ & 0,046 \\
Filhos & $-0,133$ & 0,036 & $-0,204$ & $-0,063$ & $-3,690$ & $<0,001$ \\
Escala & 9,660 & & & & & \\
\hline
\end{tabular}

O QIC obtido foi de -2872, 569 e a matriz de correlação estimada é descrita na Tabela 4.47.

Tabela 4.47: Matriz trabalho estimada referente ao ajuste no SAS do modelo EEG-Poisson aos dados de pacientes do German Health Reform.

$$
\left[\begin{array}{ccccc}
1 & 0,311 & 0,311 & 0,311 & 0,311 \\
0,311 & 1 & 0,311 & 0,311 & 0,311 \\
0,311 & 0,311 & 1 & 0,311 & 0,311 \\
0,311 & 0,311 & 0,311 & 1 & 0,311 \\
0,311 & 0,311 & 0,311 & 0,311 & 1
\end{array}\right] .
$$

Assumindo que pode existir uma sobredispersão, vamos ajustar um modelo EEG-BN com as seguintes suposições:

- $y_{i j} \sim \mathrm{BN}\left(\mu_{i j}, \nu\right), \quad i=1, \ldots, 6127$ e $j=1984, \ldots, 1988$

- $\log \left(\mu_{i j}\right)=\beta_{0}+\beta_{1} H_{i j}+\beta_{2} I_{i j}+\beta_{3} T_{i j}+\beta_{4} S_{i j}+\beta_{5} N E 2_{i j}+\beta_{6} N E 3_{i j}+\beta_{7} N E 4_{i j}+\beta_{8} C_{i j}+\beta_{9} F_{i j}$;

- $\operatorname{Corr}\left(y_{i j}, y_{i j^{\prime}}\right)=\rho$ para $j \neq j^{\prime}$ e $\operatorname{Corr}\left(y_{i j}, y_{i j^{\prime}}\right)=1$ para $j=j^{\prime}$.

Tabela 4.48: Estimativas dos parâmetros do modelo EGG-BN ajustado no SAS aos dados de pacientes do German Health Reform.

\begin{tabular}{c|r|r|r|r|r|r}
\hline Efeito & Estimativa & Erro Padrão & \multicolumn{2}{|c|}{ IC $95 \%$} & Valor-z & Valor-p \\
\hline Intercepto & 0,241 & 0,079 & 0,086 & 0,397 & 3,050 & 0,002 \\
NVHospital & 0,220 & 0,012 & 0,196 & 0,244 & 17,790 & $<0,001$ \\
Idade & 0,018 & 0,002 & 0,014 & 0,021 & 10,760 & $<0,001$ \\
Trabalhando & 0,159 & 0,034 & 0,092 & 0,226 & 4,660 & $<0,001$ \\
Feminino & 0,265 & 0,036 & 0,194 & 0,335 & 7,350 & $<0,001$ \\
NE2 & $-0,068$ & 0,069 & $-0,204$ & 0,068 & $-0,980$ & 0,325 \\
NE3 & $-0,207$ & 0,059 & $-0,322$ & $-0,091$ & $-3,510$ & $<0,001$ \\
NE4 & $-0,347$ & 0,069 & $-0,482$ & $-0,211$ & $-5,020$ & $<0,001$ \\
Casado & $-0,055$ & 0,039 & $-0,132$ & 0,022 & $-1,390$ & 0,163 \\
Filhos & $-0,131$ & 0,035 & $-0,199$ & $-0,062$ & $-3,750$ & $<0,001$ \\
Escala & 1,343 & & & & & \\
\hline
\end{tabular}

O QIC obtido foi de -69693, 901 e matriz de correlação estimada é descrita na Tabela 4.49. O SAS não forneceu a estimativa $\hat{\nu}$. 
Tabela 4.49: Matriz trabalho estimada referente ao ajuste no $S A S$ do modelo EEG-BN aos dados de pacientes do German Health Reform.

$$
\left[\begin{array}{ccccc}
1 & 0,283 & 0,283 & 0,283 & 0,283 \\
0,283 & 1 & 0,283 & 0,283 & 0,283 \\
0,283 & 0,283 & 1 & 0,283 & 0,283 \\
0,283 & 0,283 & 0,283 & 1 & 0,283 \\
0,283 & 0,283 & 0,283 & 0,283 & 1
\end{array}\right] .
$$

Vamos verificar que valores de QIC são encontrados ajustando o modelo EEG-BN com outras matrizes de correlação. O resultado é descrito abaixo:

- Simétrica: -69693, 901;

- Independente: -70213, 481;

- $\operatorname{AR}(1):-68885,725$;

- Não-estruturada: -69144,901.

Pelo visto, o modelo com a matriz de correlação independente é o que incorpora melhor a correlação entre as observações dos pacientes que na caso é nula. As estimativas obtidas sob esse modelo estão descritas na Tabela 4.50.

Tabela 4.50: Estimativas dos parâmetros do modelo EGG-BN ajustado no SAS aos dados de pacientes do German Health Reform, com matriz de correlação Independente.

\begin{tabular}{c|r|r|r|r|r|r}
\hline Efeito & Estimativa & Erro Padrão & \multicolumn{2}{|c|}{ IC 95\% } & Valor-z & Valor-p \\
\hline Intercepto & 0,221 & 0,064 & 0,096 & 0,347 & 3,450 & 0,001 \\
NVHospital & 0,399 & 0,013 & 0,375 & 0,424 & 31,870 & $<0,001$ \\
Idade & 0,017 & 0,001 & 0,014 & 0,019 & 12,800 & $<0,001$ \\
Trabalhando & 0,192 & 0,030 & 0,133 & 0,250 & 6,390 & $<0,001$ \\
Feminino & 0,256 & 0,028 & 0,201 & 0,311 & 9,130 & $<0,001$ \\
NE2 & $-0,081$ & 0,054 & $-0,188$ & 0,025 & $-1,500$ & 0,134 \\
NE3 & $-0,207$ & 0,046 & $-0,298$ & $-0,116$ & $-4,460$ & $<0,001$ \\
NE4 & $-0,379$ & 0,054 & $-0,485$ & $-0,274$ & $-7,050$ & $<0,001$ \\
Casado & $-0,023$ & 0,033 & $-0,086$ & 0,041 & $-0,690$ & 0,489 \\
Filhos & $-0,158$ & 0,030 & $-0,216$ & $-0,100$ & $-5,330$ & $<0,001$ \\
Escala & 1,334 & & & & & \\
\hline
\end{tabular}

Os resultados são idênticos aos que são encontrados para o modelo log-linear com resposta binomial negativa, o que era de se esperar já que foi encontrado que a matriz de correlação é independente.

\subsubsection{Melhorando o Modelo}

Como não foi possível aplicar técnicas de diagnóstico para avaliar se o modelo estava bem ajustado aos dados, devido ao número grande de dados, a única forma de melhorar o modelo é retirando as variáveis que apresentam um valor-p maior do que um nível de significância de 10\%, como podemos ver na tabela 4.50. Essas variáveis são $N E 2$ e Casado. Como NE2 é um fator com vários níveis que parecem ser significativos, não há razões para retirar essa variável do modelo. Vamos reajustar o modelo EEG-BN sem a variável Casado. As estimativas dos parâmetros desse modelo se encontram na Tabela 4.51. 
Tabela 4.51: Estimativas dos parâmetros do modelo EGG-BN ajustado no SAS aos dados de pacientes do German Health Reform, com matriz de correlação Independente e sem a variável Casado.

\begin{tabular}{c|r|r|r|r|r|r}
\hline Efeito & Estimativa & Erro Padrão & \multicolumn{2}{|c|}{ IC 95\% } & Valor-z & Valor-p \\
\hline Intercepto & 0,216 & 0,064 & 0,091 & 0,341 & 3,390 & 0,001 \\
NVHospital & 0,400 & 0,013 & 0,375 & 0,424 & 31,900 & $<0,001$ \\
Idade & 0,017 & 0,001 & 0,014 & 0,019 & 13,060 & $<0,001$ \\
Trabalhando & 0,192 & 0,030 & 0,134 & 0,251 & 6,410 & $<0,001$ \\
Feminino & 0,258 & 0,028 & 0,203 & 0,313 & 9,190 & $<0,001$ \\
NE2 & $-0,081$ & 0,054 & $-0,188$ & 0,025 & $-1,500$ & 0,134 \\
NE3 & $-0,205$ & 0,046 & $-0,296$ & $-0,114$ & $-4,430$ & $<0,001$ \\
NE4 & $-0,377$ & 0,054 & $-0,482$ & $-0,271$ & $-7,010$ & $<0,001$ \\
Filhos & $-0,165$ & 0,028 & $-0,220$ & $-0,110$ & $-5,890$ & $<0,001$ \\
Escala & 1,334 & & & & & \\
\hline
\end{tabular}

\subsubsection{Conclusões}

O modelo final que foi escolhido foi o seguinte:

- $y_{i j} \sim \mathrm{BN}\left(\mu_{i j}, \nu\right), \quad i=1, \ldots, 6127 \quad$ e $j=1984, \ldots, 1988$

- $\log \left(\mu_{i j}\right)=\beta_{0}+\beta_{1} H_{i j}+\beta_{2} I_{i j}+\beta_{3} T_{i j}+\beta_{4} S_{i j}+\beta_{5} N E 2_{i j}+\beta_{6} N E 3_{i j}+\beta_{7} N E 4_{i j}+\beta_{9} F_{i j}$

- $\operatorname{Corr}\left(y_{i j}, y_{i j^{\prime}}\right)=0$ para $j \neq j^{\prime}$ e $\operatorname{Corr}\left(y_{i j}, y_{i j^{\prime}}\right)=1$ para $j=j^{\prime}$,

cujas estimativas dos parâmetros são apresentadas na Tabela 4.51.

Para facilitar a apresentação vamos interpretar os valores estimados para os parâmetros, por meio da razão de médias que pode ser derivada facilmente através de modelos log-lineares. Temos as seguintes estimativas:

- Número de visitas ao hospital. Com uma razão de médias de 1,490 (IC95\%: 1,460;1,522), vemos que a cada aumento de uma unidade no número de visitas ao hospital o número médio de visitas ao médico aumenta em 49\%. O que contradiz a tendência da Figura 4.23.

- Idade do paciente. Com uma razão de médias de 1,017 (IC95\%: 1,014;1,020), vemos que a cada aumento de uma unidade na idade do paciente o número médio de visitas ao médico aumenta $1,7 \%$.

- Paciente estava trabalhando. Com uma razão de médias de 1,211 (IC95\%: 1,124;1,305), vemos que pacientes que estavam trabalhando têm ido ao médico em média $21 \%$ mais vezes do que os que não estão trabalhando.

- Sexo feminino. Com uma razão de médias de 1,292 (IC95\%: 1,196;1,396), vemos que mulheres vão em média ao médico $29 \%$ vezes a mais do que os homens.

- Nível Escolar 2. Com uma razão de médias de 0,922 (IC95\%: 0,819;1,037), vemos que pessoas com um nível escolar 2 têm em média um número de visitas ao médico $8 \%$ menor do que os pacientes com nível escolar 1.

- Nível Escolar 3. Com uma razão de médias de 0,813 (IC95\%: 0,727;0,910), vemos que pessoas com um nível escolar 3 têm em média um número de visitas ao médico $19 \%$ menor do que os pacientes com nível escolar 1 .

- Nível Escolar 4. Com uma razão de médias de 0,684 (IC95\%: 0,598;0,783), vemos que pessoas com um nível escolar 4 têm em média um número de visitas ao médico $32 \%$ menor do que os pacientes com nível escolar 1. 
- Filhos. Com uma razão de médias de 0,854 (IC95\%: 0,792;0,920), vemos que pessoas com filhos têm em média um número de visitas ao médico $15 \%$ menor do que os pacientes sem filhos.

De modo geral podemos dizer que o número de visitas ao hospital, idade do paciente, se o paciente estava trabalhando e o sexo do paciente têm influência diretamente proporcional no número de visitas ao médico, ou seja, aumentam o número médio de visitas ao médico. Enquanto que as variáveis nível escolar, se o paciente estava casado e se tinha filhos, influenciam de forma inversamente proporcional, ou seja, diminuem o número médio de visitas ao médico. Esses resultados vão ao encontro das análises descritivas, exceto pela variável número de visitas ao hospital.

Hilbe (2011) trabalhou um capítulo somente usando o banco de dados para ilustrar os métodos, e utilizou apenas as variáveis sexo do paciente e nível educacional. Mesmo assim podemos comparar os resultados encontrados para essas variáveis. E o que foi encontrado por Hilbe (2011) foi similar ao que foi encontrado em nosso estudo, ou seja, a variável sexo do paciente aumentou o número de visitas ao médico e o nível educacional diminuiu o número médio de visitas ao médico.

\subsection{Método Alternativo}

Como foi visto no texto, uma sugestão para o ajuste de um modelo EEG-BN no R é através de cópulas Gaussianas. Vamos verificar como esse modelo se comporta para o nosso último banco de dados. Primeiro, vamos ajustar o modelo de cópulas Gaussianas com resposta Poisson. As estimativas são apresentadas na Tabela 4.52.

Tabela 4.52: Estimativas dos parâmetros do modelo de cópulas Gaussianas com resposta Poisson ajustado no $R$ aos dados de pacientes do German Health Reform.

\begin{tabular}{c|r|r|r|r}
\hline Efeito & Estimativa & Erro Padrão & Valor-z & Valor-p \\
\hline Intercepto & 0,144 & 0,024 & 6010,000 & $<0,001$ \\
NVHospital & 0,120 & 0,004 & 32633,000 & $<0,001$ \\
Idade & 0,015 & 0,000 & 31786,000 & $<0,001$ \\
Trabalhando & 0,189 & 0,009 & 20270,000 & $<0,001$ \\
Feminino & 0,243 & 0,008 & 30983,000 & $<0,001$ \\
NE2 & 0,017 & 0,023 & 0,746 & 0,456 \\
NE3 & $-0,145$ & 0,020 & $-7310,000$ & $<0,001$ \\
NE44 & $-0,282$ & 0,026 & $-11025,000$ & $<0,001$ \\
Casado & $-0,014$ & 0,009 & $-1520,000$ & 0,128 \\
Filhos & $-0,149$ & 0,010 & $-14532,000$ & $<0,001$ \\
\hline
\end{tabular}

Obtivemos um AIC de 118144. O programa demorou 38 minutos para chegar a esses valores, o que pode ser explicado pelo tamanho do banco de dados. Vamos verificar qual o resultado para o modelo com resposta binomial negativa, com matriz de correlação simétrica, cujos resultados são apresentados na Tabela 4.53 .

Obtivemos um AIC de 82351. Para esse ajuste o programa demorou 33 minutos. Esses resultados são muito similares do ponto de vista inferencial aos resultados descritos na Tabela 4.48. Vamos verificar se a escolha da melhor matriz trabalho também coincide com o ajuste do modelo EEG-BNl, comparando os valores do AIC:

- Simétrica: 82351;

- Independente: 85237;

- $\operatorname{AR}(1): 82859$. 
Tabela 4.53: Estimativas dos parâmetros do modelo de cópulas Gaussianas com resposta BN ajustado no $R$ aos dados de pacientes do German Health Reform.

\begin{tabular}{c|r|r|r|r}
\hline Efeito & Estimativa & Erro Padrão & Valor-z & Valor-p \\
\hline Intercepto & 0,462 & 0,068 & 6809,000 & $<0,001$ \\
NVHospital & 0,200 & 0,020 & 10075,000 & $<0,001$ \\
Idade & 0,014 & 0,001 & 10487,000 & $<0,001$ \\
Trabalhando & 0,102 & 0,028 & 3695,000 & $<0,001$ \\
Feminino & 0,174 & 0,030 & 5865,000 & $<0,001$ \\
NE2 & $-0,144$ & 0,058 & $-2480,000$ & 0,013 \\
NE3 & $-0,215$ & 0,050 & $-4330,000$ & $<0,001$ \\
NE44 & $-0,379$ & 0,058 & $-6527,000$ & $<0,001$ \\
Casado & $-0,058$ & 0,033 & $-1766,000$ & 0,077 \\
Filhos & $-0,097$ & 0,029 & $-3323,000$ & 0,001 \\
$\hat{\nu}$ & 1,996 & 0,033 & 60,817 & $<0,001$ \\
\hline
\end{tabular}

Não foi possível obter os resultados para a matriz de correlação não estruturada, pois o programa demorou muito para rodar. Como podemos verificar, a matriz de correlação que parece se ajustar melhor aos dados é a simétrica. O que difere dos resultados encontrados pelo ajuste do modelo EEG-BN.

Assim, podemos concluir que o método alternativo de cópulas Gaussianas com resposta binomial negativa leva aos mesmos resultados inferenciais do ajuste do modelo EEG-BN. Contudo, sob o modelo de cópulas Gaussianas tem-se erros padrões bem menores e o modelo com menor valor de AIC contempla a estrutura de correlação simétrica. Talvez o fato da matriz trabalho estimada sob o modelo EEG-BN poduzir uma estimativa baixa para a correlação, não tenha produzido mudanças inferenciais importantes entre as duas metodologias. 


\section{Capítulo 5}

\section{Conclusões}

O objetivo principal deste trabalho foi dissertar sobre EEGs aplicadas a dados de contagem correlacionados e com sobredispersão e verificar a sua aplicação em exemplos de diversos níveis de dificuldade. Na parte teórica do trabalho revisamos os principais conceitos que envolvem EEGs, tanto para o caso geral, que é para todos os tipos de dados pertencentes à família exponencial, como também para o caso de modelos com resposta binomial negativa.

Na parte específica de extensão para a binomial negativa, foram expostos os tópicos que foram encontrados na literatura sobre o assunto. Geralmente, o que é feito, é a estimação do parâmetro de dispersão da BN por máxima verossimilhança, sem levar em conta a correlação e utilizar esse valor como constante nos softwares e com isso tratar a BN como se fosse da FE. Essa é a forma como as funções dos softwares SAS e Stata tratam da estimação do modelo EEG-BN. Não foi encontrada nenhuma função no R que fizesse essa estimação. Houve uma tentativa de criar essa função, para esta dissertação, mas o desenvolvimento do código foi mais complicado do que o previsto.

Como uma forma de tentar solucionar a ausência de função no $\mathrm{R}$ que resolva modelos de contagem correlacionados e com sobredispersão, foi encontrada uma alternativa através do método de cópulas Gaussianas. A teoria sobre esse assunto não foi muito desenvolvida, para não destoar muito do principal. No entanto, essa metodologia foi colocada em prática com os exemplos estudados.

No início do estudo foi proposto analisar a modelagem em três tipos diferentes de bancos de dados. Um banco fácil (pacientes com lepra), que possuía dados balanceados e poucos pacientes. Nesse banco, todos as funções rodaram e convergiram e foi possível fazer uma boa análise, apesar da baixa quantidade de observações.

Houve um banco de dados de dificuldade mediana (pacientes com epilepsia), que apesar de possuir um número maior de observações por paciente e mais pacientes, havia a questão de uma semana de base, que foi preciso contornar. Esse banco é o que apresentou mais aplicações na literatura tendo um bom suporte para comparação.

O último banco pode ser considerado difícil de analisar (pacientes do German Health Reform), pois possui um grande número de pacientes e observações desbalanceadas, além de muitas variáveis explicativas. De fato, o tamanho do banco atrapalhou a análise, principalmente na parte de diagnóstico do ajuste dos modelos, além da demora para rodar as funções.

Em todos os bancos, havia características que nos faziam acreditar que o melhor modelo seria aquele que levasse em conta a resposta de contagem, a sobredispersão e a correlação entre as observações agrupadas. Nos dois primeiros exemplos essa suposição foi comprovada, e o melhor modelo foi o EEG-BN com matriz de correlação simétrica, que foi ajustado pelo SAS. Já no último exemplo, o melhor modelo foi o MLG-BN, ou seja, que ajustou somente a sobredispersão e a contagem.

Nos três exemplos os melhores modelos foram escolhidos baseados nos valores de QIC. E todas as análises foram feitas no R ou no SAS.

Em relação à modelagem utilizando a metodologia alternativa de cópulas Gaussianas, em todos os exemplos o resultado final encontrado foi diferente. Algumas vezes o que diferenciou foram as estruturas de correlação, em outros foram as variáveis que foram escolhidas como significativas para 
o modelo. Então, de forma geral, podemos concluir que embora as cópulas Gaussianas sejam uma forma alternativa de análise esse tipo de análise nem sempre levam aos mesmos resultados das EEGs.

\subsection{Considerações Finais}

Apesar de ainda haver muito o que desenvolver, tanto na parte teórica quanto na parte de aplicação em softwares, o modelo EEG-BN, apresentou ser uma forma adequada de ajustar dados correlacionados com resposta de contagem e com sobredispersão.

\subsection{Sugestões para Pesquisas Futuras}

Ainda há muito o que ser desenvolvido em pesquisas futuras para EEGs com resposta binomial negativa. Por exemplo, a sua aplicação em softwares mais utilizados, como o $\mathrm{R}$, ainda que na sua forma da FE. Também é preciso desenvolver mais alternativas para o EEG-BN, como por exemplo, através de EEG2 ou EEG3, em que o parâmetro de dispersão também é levado em conta em conta bem como a estrutura de correlação na modelagem através de EEGs. 


\section{Apêndice A}

\section{Bancos de dados completos}

\section{A.1 Leprosy}

Tabela A.1: Leprosy: Banco de dados

\begin{tabular}{|c|c|c|c|c|c|c|c|}
\hline id & count & time & drug & id & count & time & drug \\
\hline 1 & 11 & 0 & 0 & 16 & 6 & 0 & 0 \\
\hline 1 & 6 & 1 & 0 & 16 & 4 & 1 & 0 \\
\hline 2 & 6 & 0 & 1 & 17 & 8 & 0 & 1 \\
\hline 2 & 0 & 1 & 1 & 17 & 4 & 1 & 1 \\
\hline 3 & 16 & 0 & 1 & 18 & 16 & 0 & 1 \\
\hline 3 & 13 & 1 & 1 & 18 & 12 & 1 & 1 \\
\hline 4 & 8 & 0 & 0 & 19 & 10 & 0 & 0 \\
\hline 4 & 0 & 1 & 0 & 19 & 13 & 1 & 0 \\
\hline 5 & 6 & 0 & 1 & 20 & 19 & 0 & 1 \\
\hline 5 & 2 & 1 & 1 & 20 & 14 & 1 & 1 \\
\hline 6 & 13 & 0 & 1 & 21 & 12 & 0 & 1 \\
\hline 6 & 10 & 1 & 1 & 21 & 5 & 1 & 1 \\
\hline 7 & 5 & 0 & 0 & 22 & 6 & 0 & 0 \\
\hline 7 & 2 & 1 & 0 & 22 & 1 & 1 & 0 \\
\hline 8 & 7 & 0 & 1 & 23 & 8 & 0 & 1 \\
\hline 8 & 3 & 1 & 1 & 23 & 9 & 1 & 1 \\
\hline 9 & 11 & 0 & 1 & 24 & 12 & 0 & 1 \\
\hline 9 & 18 & 1 & 1 & 24 & 16 & 1 & 1 \\
\hline 10 & 14 & 0 & 0 & 25 & 11 & 0 & 0 \\
\hline 10 & 8 & 1 & 0 & 25 & 8 & 1 & 0 \\
\hline 11 & 8 & 0 & 1 & 26 & 5 & 0 & 1 \\
\hline 11 & 1 & 1 & 1 & 26 & 1 & 1 & 1 \\
\hline 12 & 9 & 0 & 1 & 27 & 7 & 0 & 1 \\
\hline 12 & 5 & 1 & 1 & 27 & 1 & 1 & 1 \\
\hline 13 & 19 & 0 & 0 & 28 & 3 & 0 & 0 \\
\hline 13 & 11 & 1 & 0 & 28 & 0 & 1 & 0 \\
\hline 14 & 18 & 0 & 1 & 29 & 15 & 0 & 1 \\
\hline 14 & 18 & 1 & 1 & 29 & 9 & 1 & 1 \\
\hline 15 & 21 & 0 & 1 & 30 & 12 & 0 & 1 \\
\hline 15 & 23 & 1 & 1 & 30 & 20 & 1 & 1 \\
\hline
\end{tabular}




\section{A.2 Progabide}

Tabela A.2: Progabide: Banco de dados

\begin{tabular}{cccccc}
\hline ID & trat & seizures & $\mathrm{t}$ & $\mathrm{m}$ & tempo \\
\hline 104 & 0 & 11 & 0 & 8 & 0 \\
104 & 0 & 5 & 1 & 2 & 1 \\
104 & 0 & 3 & 2 & 2 & 1 \\
104 & 0 & 3 & 3 & 2 & 1 \\
104 & 0 & 3 & 4 & 2 & 1 \\
106 & 0 & 11 & 0 & 8 & 0 \\
106 & 0 & 3 & 1 & 2 & 1 \\
106 & 0 & 5 & 2 & 2 & 1 \\
106 & 0 & 3 & 3 & 2 & 1 \\
106 & 0 & 3 & 4 & 2 & 1 \\
107 & 0 & 6 & 0 & 8 & 0 \\
107 & 0 & 2 & 1 & 2 & 1 \\
107 & 0 & 4 & 2 & 2 & 1 \\
107 & 0 & 0 & 3 & 2 & 1 \\
107 & 0 & 5 & 4 & 2 & 1 \\
114 & 0 & 8 & 0 & 8 & 0 \\
114 & 0 & 4 & 1 & 2 & 1 \\
114 & 0 & 4 & 2 & 2 & 1 \\
114 & 0 & 1 & 3 & 2 & 1 \\
114 & 0 & 4 & 4 & 2 & 1 \\
116 & 0 & 66 & 0 & 8 & 0 \\
116 & 0 & 7 & 1 & 2 & 1 \\
116 & 0 & 18 & 2 & 2 & 1 \\
116 & 0 & 9 & 3 & 2 & 1 \\
116 & 0 & 21 & 4 & 2 & 1 \\
118 & 0 & 27 & 0 & 8 & 0 \\
118 & 0 & 5 & 1 & 2 & 1 \\
118 & 0 & 2 & 2 & 2 & 1 \\
118 & 0 & 8 & 3 & 2 & 1 \\
118 & 0 & 7 & 4 & 2 & 1 \\
123 & 0 & 12 & 0 & 8 & 0 \\
123 & 0 & 6 & 1 & 2 & 1 \\
123 & 0 & 4 & 2 & 2 & 1 \\
123 & 0 & 0 & 3 & 2 & 1 \\
123 & 0 & 2 & 4 & 2 & 1 \\
126 & 0 & 52 & 0 & 8 & 0 \\
126 & 0 & 40 & 1 & 2 & 1 \\
126 & 0 & 20 & 2 & 2 & 1 \\
126 & 0 & 23 & 3 & 2 & 1 \\
126 & 0 & 12 & 4 & 2 & 1 \\
130 & 0 & 23 & 0 & 8 & 0 \\
130 & 0 & 5 & 1 & 2 & 1 \\
130 & 0 & 6 & 2 & 2 & 1 \\
130 & 0 & 6 & 3 & 2 & 1 \\
130 & 0 & 5 & 4 & 2 & 1 \\
135 & 0 & 10 & 0 & 8 & 0 \\
\hline & Continua na & próxima página \\
\hline & & & & \\
\end{tabular}


Tabela A.2 - continuação

\begin{tabular}{cccccc}
\hline ID & trat & seizures & $\mathrm{t}$ & $\mathrm{m}$ & tempo \\
\hline 135 & 0 & 14 & 1 & 2 & 1 \\
135 & 0 & 13 & 2 & 2 & 1 \\
135 & 0 & 6 & 3 & 2 & 1 \\
135 & 0 & 0 & 4 & 2 & 1 \\
141 & 0 & 52 & 0 & 8 & 0 \\
141 & 0 & 26 & 1 & 2 & 1 \\
141 & 0 & 12 & 2 & 2 & 1 \\
141 & 0 & 6 & 3 & 2 & 1 \\
141 & 0 & 22 & 4 & 2 & 1 \\
145 & 0 & 33 & 0 & 8 & 0 \\
145 & 0 & 12 & 1 & 2 & 1 \\
145 & 0 & 6 & 2 & 2 & 1 \\
145 & 0 & 8 & 3 & 2 & 1 \\
145 & 0 & 5 & 4 & 2 & 1 \\
201 & 0 & 18 & 0 & 8 & 0 \\
201 & 0 & 4 & 1 & 2 & 1 \\
201 & 0 & 4 & 2 & 2 & 1 \\
201 & 0 & 6 & 3 & 2 & 1 \\
201 & 0 & 2 & 4 & 2 & 1 \\
202 & 0 & 42 & 0 & 8 & 0 \\
202 & 0 & 7 & 1 & 2 & 1 \\
202 & 0 & 9 & 2 & 2 & 1 \\
202 & 0 & 12 & 3 & 2 & 1 \\
202 & 0 & 14 & 4 & 2 & 1 \\
205 & 0 & 87 & 0 & 8 & 0 \\
205 & 0 & 16 & 1 & 2 & 1 \\
205 & 0 & 24 & 2 & 2 & 1 \\
205 & 0 & 10 & 3 & 2 & 1 \\
205 & 0 & 9 & 4 & 2 & 1 \\
206 & 0 & 50 & 0 & 8 & 0 \\
206 & 0 & 11 & 1 & 2 & 1 \\
206 & 0 & 0 & 2 & 2 & 1 \\
206 & 0 & 0 & 3 & 2 & 1 \\
206 & 0 & 5 & 4 & 2 & 1 \\
210 & 0 & 18 & 0 & 8 & 0 \\
210 & 0 & 0 & 1 & 2 & 1 \\
210 & 0 & 0 & 2 & 2 & 1 \\
210 & 0 & 3 & 3 & 2 & 1 \\
210 & 0 & 3 & 4 & 2 & 1 \\
213 & 0 & 111 & 0 & 8 & 0 \\
213 & 0 & 37 & 1 & 2 & 1 \\
213 & 0 & 29 & 2 & 2 & 1 \\
213 & 0 & 28 & 3 & 2 & 1 \\
213 & 0 & 29 & 4 & 2 & 1 \\
215 & 0 & 18 & 0 & 8 & 0 \\
215 & 0 & 3 & 1 & 2 & 1 \\
215 & 0 & 5 & 2 & 2 & 1 \\
215 & 0 & 2 & 3 & 2 & 1 \\
215 & 0 & 5 & 4 & 2 & 1 \\
\hline & Continua na próxima & página \\
\hline & & & & & \\
\hline
\end{tabular}


Tabela A.2 - continuação

\begin{tabular}{cccccc}
\hline ID & trat & seizures & $\mathrm{t}$ & $\mathrm{m}$ & tempo \\
\hline 217 & 0 & 20 & 0 & 8 & 0 \\
217 & 0 & 3 & 1 & 2 & 1 \\
217 & 0 & 0 & 2 & 2 & 1 \\
217 & 0 & 6 & 3 & 2 & 1 \\
217 & 0 & 7 & 4 & 2 & 1 \\
219 & 0 & 12 & 0 & 8 & 0 \\
219 & 0 & 3 & 1 & 2 & 1 \\
219 & 0 & 4 & 2 & 2 & 1 \\
219 & 0 & 3 & 3 & 2 & 1 \\
219 & 0 & 4 & 4 & 2 & 1 \\
220 & 0 & 9 & 0 & 8 & 0 \\
220 & 0 & 3 & 1 & 2 & 1 \\
220 & 0 & 4 & 2 & 2 & 1 \\
220 & 0 & 3 & 3 & 2 & 1 \\
220 & 0 & 4 & 4 & 2 & 1 \\
222 & 0 & 17 & 0 & 8 & 0 \\
222 & 0 & 2 & 1 & 2 & 1 \\
222 & 0 & 3 & 2 & 2 & 1 \\
222 & 0 & 3 & 3 & 2 & 1 \\
222 & 0 & 5 & 4 & 2 & 1 \\
226 & 0 & 28 & 0 & 8 & 0 \\
226 & 0 & 8 & 1 & 2 & 1 \\
226 & 0 & 12 & 2 & 2 & 1 \\
226 & 0 & 2 & 3 & 2 & 1 \\
226 & 0 & 8 & 4 & 2 & 1 \\
227 & 0 & 55 & 0 & 8 & 0 \\
227 & 0 & 18 & 1 & 2 & 1 \\
227 & 0 & 24 & 2 & 2 & 1 \\
227 & 0 & 76 & 3 & 2 & 1 \\
227 & 0 & 25 & 4 & 2 & 1 \\
230 & 0 & 9 & 0 & 8 & 0 \\
230 & 0 & 2 & 1 & 2 & 1 \\
230 & 0 & 1 & 2 & 2 & 1 \\
230 & 0 & 2 & 3 & 2 & 1 \\
230 & 0 & 1 & 4 & 2 & 1 \\
234 & 0 & 10 & 0 & 8 & 0 \\
234 & 0 & 3 & 1 & 2 & 1 \\
234 & 0 & 1 & 2 & 2 & 1 \\
234 & 0 & 4 & 3 & 2 & 1 \\
234 & 0 & 2 & 4 & 2 & 1 \\
238 & 0 & 47 & 0 & 8 & 0 \\
238 & 0 & 13 & 1 & 2 & 1 \\
238 & 0 & 15 & 2 & 2 & 1 \\
238 & 0 & 13 & 3 & 2 & 1 \\
238 & 0 & 12 & 4 & 2 & 1 \\
101 & 1 & 76 & 0 & 8 & 0 \\
101 & 1 & 11 & 1 & 2 & 1 \\
101 & 1 & 14 & 2 & 2 & 1 \\
101 & 1 & 9 & 3 & 2 & 1 \\
\hline & Continua na próxima página \\
\hline & & & & \\
\end{tabular}


Tabela A.2 - continuação

\begin{tabular}{|c|c|c|c|c|c|}
\hline ID & trat & seizures & $\mathrm{t}$ & $\mathrm{m}$ & tempo \\
\hline 101 & 1 & 8 & 4 & 2 & 1 \\
\hline 102 & 1 & 38 & 0 & 8 & 0 \\
\hline 102 & 1 & 8 & 1 & 2 & 1 \\
\hline 102 & 1 & 7 & 2 & 2 & 1 \\
\hline 102 & 1 & 9 & 3 & 2 & 1 \\
\hline 102 & 1 & 4 & 4 & 2 & 1 \\
\hline 103 & 1 & 19 & 0 & 8 & 0 \\
\hline 103 & 1 & 0 & 1 & 2 & 1 \\
\hline 103 & 1 & 4 & 2 & 2 & 1 \\
\hline 103 & 1 & 3 & 3 & 2 & 1 \\
\hline 103 & 1 & 0 & 4 & 2 & 1 \\
\hline 108 & 1 & 10 & 0 & 8 & 0 \\
\hline 108 & 1 & 3 & 1 & 2 & 1 \\
\hline 108 & 1 & 6 & 2 & 2 & 1 \\
\hline 108 & 1 & 1 & 3 & 2 & 1 \\
\hline 108 & 1 & 3 & 4 & 2 & 1 \\
\hline 110 & 1 & 19 & 0 & 8 & 0 \\
\hline 110 & 1 & 2 & 1 & 2 & 1 \\
\hline 110 & 1 & 6 & 2 & 2 & 1 \\
\hline 110 & 1 & 7 & 3 & 2 & 1 \\
\hline 110 & 1 & 4 & 4 & 2 & 1 \\
\hline 111 & 1 & 24 & 0 & 8 & 0 \\
\hline 111 & 1 & 4 & 1 & 2 & 1 \\
\hline 111 & 1 & 3 & 2 & 2 & 1 \\
\hline 111 & 1 & 1 & 3 & 2 & 1 \\
\hline 111 & 1 & 3 & 4 & 2 & 1 \\
\hline 112 & 1 & 31 & 0 & 8 & 0 \\
\hline 112 & 1 & 22 & 1 & 2 & 1 \\
\hline 112 & 1 & 17 & 2 & 2 & 1 \\
\hline 112 & 1 & 19 & 3 & 2 & 1 \\
\hline 112 & 1 & 16 & 4 & 2 & 1 \\
\hline 113 & 1 & 14 & 0 & 8 & 0 \\
\hline 113 & 1 & 5 & 1 & 2 & 1 \\
\hline 113 & 1 & 4 & 2 & 2 & 1 \\
\hline 113 & 1 & 7 & 3 & 2 & 1 \\
\hline 113 & 1 & 4 & 4 & 2 & 1 \\
\hline 117 & 1 & 11 & 0 & 8 & 0 \\
\hline 117 & 1 & 2 & 1 & 2 & 1 \\
\hline 117 & 1 & 4 & 2 & 2 & 1 \\
\hline 117 & 1 & 0 & 3 & 2 & 1 \\
\hline 117 & 1 & 4 & 4 & 2 & 1 \\
\hline 121 & 1 & 67 & 0 & 8 & 0 \\
\hline 121 & 1 & 3 & 1 & 2 & 1 \\
\hline 121 & 1 & 7 & 2 & 2 & 1 \\
\hline 121 & 1 & 7 & 3 & 2 & 1 \\
\hline 121 & 1 & 7 & 4 & 2 & 1 \\
\hline 122 & 1 & 41 & 0 & 8 & 0 \\
\hline 122 & 1 & 4 & 1 & 2 & 1 \\
\hline 122 & 1 & 18 & 2 & 2 & 1 \\
\hline
\end{tabular}


Tabela A.2 - continuação

\begin{tabular}{cccccc}
\hline ID & trat & seizures & $\mathrm{t}$ & $\mathrm{m}$ & tempo \\
\hline 122 & 1 & 2 & 3 & 2 & 1 \\
122 & 1 & 5 & 4 & 2 & 1 \\
124 & 1 & 7 & 0 & 8 & 0 \\
124 & 1 & 2 & 1 & 2 & 1 \\
124 & 1 & 1 & 2 & 2 & 1 \\
124 & 1 & 1 & 3 & 2 & 1 \\
124 & 1 & 0 & 4 & 2 & 1 \\
128 & 1 & 22 & 0 & 8 & 0 \\
128 & 1 & 0 & 1 & 2 & 1 \\
128 & 1 & 2 & 2 & 2 & 1 \\
128 & 1 & 4 & 3 & 2 & 1 \\
128 & 1 & 0 & 4 & 2 & 1 \\
129 & 1 & 13 & 0 & 8 & 0 \\
129 & 1 & 5 & 1 & 2 & 1 \\
129 & 1 & 4 & 2 & 2 & 1 \\
129 & 1 & 0 & 3 & 2 & 1 \\
129 & 1 & 3 & 4 & 2 & 1 \\
137 & 1 & 46 & 0 & 8 & 0 \\
137 & 1 & 11 & 1 & 2 & 1 \\
137 & 1 & 14 & 2 & 2 & 1 \\
137 & 1 & 25 & 3 & 2 & 1 \\
137 & 1 & 15 & 4 & 2 & 1 \\
139 & 1 & 36 & 0 & 8 & 0 \\
139 & 1 & 10 & 1 & 2 & 1 \\
139 & 1 & 5 & 2 & 2 & 1 \\
139 & 1 & 3 & 3 & 2 & 1 \\
139 & 1 & 8 & 4 & 2 & 1 \\
143 & 1 & 38 & 0 & 8 & 0 \\
143 & 1 & 19 & 1 & 2 & 1 \\
143 & 1 & 7 & 2 & 2 & 1 \\
143 & 1 & 6 & 3 & 2 & 1 \\
143 & 1 & 7 & 4 & 2 & 1 \\
147 & 1 & 7 & 0 & 8 & 0 \\
147 & 1 & 1 & 1 & 2 & 1 \\
147 & 1 & 1 & 2 & 2 & 1 \\
147 & 1 & 2 & 3 & 2 & 1 \\
147 & 1 & 4 & 4 & 2 & 1 \\
203 & 1 & 36 & 0 & 8 & 0 \\
203 & 1 & 6 & 1 & 2 & 1 \\
203 & 1 & 10 & 2 & 2 & 1 \\
203 & 1 & 8 & 3 & 2 & 1 \\
203 & 1 & 8 & 4 & 2 & 1 \\
204 & 1 & 11 & 0 & 8 & 0 \\
204 & 1 & 2 & 1 & 2 & 1 \\
204 & 1 & 1 & 2 & 2 & 1 \\
204 & 1 & 0 & 3 & 2 & 1 \\
204 & 1 & 0 & 4 & 2 & 1 \\
207 & 1 & 151 & 0 & 8 & 0 \\
207 & 1 & 102 & 1 & 2 & 1 \\
\hline & Continua na próxima página \\
\hline & & & & \\
\end{tabular}


Tabela A.2 - continuação

\begin{tabular}{|c|c|c|c|c|c|}
\hline ID & trat & seizures & $\mathrm{t}$ & $\mathrm{m}$ & tempo \\
\hline 207 & 1 & 65 & 2 & 2 & 1 \\
\hline 207 & 1 & 72 & 3 & 2 & 1 \\
\hline 207 & 1 & 63 & 4 & 2 & 1 \\
\hline 208 & 1 & 22 & 0 & 8 & 0 \\
\hline 208 & 1 & 4 & 1 & 2 & 1 \\
\hline 208 & 1 & 3 & 2 & 2 & 1 \\
\hline 208 & 1 & 2 & 3 & 2 & 1 \\
\hline 208 & 1 & 4 & 4 & 2 & 1 \\
\hline 209 & 1 & 42 & 0 & 8 & 0 \\
\hline 209 & 1 & 8 & 1 & 2 & 1 \\
\hline 209 & 1 & 6 & 2 & 2 & 1 \\
\hline 209 & 1 & 5 & 3 & 2 & 1 \\
\hline 209 & 1 & 7 & 4 & 2 & 1 \\
\hline 211 & 1 & 32 & 0 & 8 & 0 \\
\hline 211 & 1 & 1 & 1 & 2 & 1 \\
\hline 211 & 1 & 3 & 2 & 2 & 1 \\
\hline 211 & 1 & 1 & 3 & 2 & 1 \\
\hline 211 & 1 & 5 & 4 & 2 & 1 \\
\hline 214 & 1 & 56 & 0 & 8 & 0 \\
\hline 214 & 1 & 18 & 1 & 2 & 1 \\
\hline 214 & 1 & 11 & 2 & 2 & 1 \\
\hline 214 & 1 & 28 & 3 & 2 & 1 \\
\hline 214 & 1 & 13 & 4 & 2 & 1 \\
\hline 218 & 1 & 24 & 0 & 8 & 0 \\
\hline 218 & 1 & 6 & 1 & 2 & 1 \\
\hline 218 & 1 & 3 & 2 & 2 & 1 \\
\hline 218 & 1 & 4 & 3 & 2 & 1 \\
\hline 218 & 1 & 0 & 4 & 2 & 1 \\
\hline 221 & 1 & 16 & 0 & 8 & 0 \\
\hline 221 & 1 & 3 & 1 & 2 & 1 \\
\hline 221 & 1 & 5 & 2 & 2 & 1 \\
\hline 221 & 1 & 4 & 3 & 2 & 1 \\
\hline 221 & 1 & 3 & 4 & 2 & 1 \\
\hline 225 & 1 & 22 & 0 & 8 & 0 \\
\hline 225 & 1 & 1 & 1 & 2 & 1 \\
\hline 225 & 1 & 23 & 2 & 2 & 1 \\
\hline 225 & 1 & 19 & 3 & 2 & 1 \\
\hline 225 & 1 & 8 & 4 & 2 & 1 \\
\hline 228 & 1 & 25 & 0 & 8 & 0 \\
\hline 228 & 1 & 2 & 1 & 2 & 1 \\
\hline 228 & 1 & 3 & 2 & 2 & 1 \\
\hline 228 & 1 & 0 & 3 & 2 & 1 \\
\hline 228 & 1 & 1 & 4 & 2 & 1 \\
\hline 232 & 1 & 13 & 0 & 8 & 0 \\
\hline 232 & 1 & 0 & 1 & 2 & 1 \\
\hline 232 & 1 & 0 & 2 & 2 & 1 \\
\hline 232 & 1 & 0 & 3 & 2 & 1 \\
\hline 232 & 1 & 0 & 4 & 2 & 1 \\
\hline 236 & 1 & 12 & 0 & 8 & 0 \\
\hline
\end{tabular}


Tabela A.2 - continuação

\begin{tabular}{cccccc}
\hline ID & trat & seizures & $\mathrm{t}$ & $\mathrm{m}$ & tempo \\
\hline 236 & 1 & 1 & 1 & 2 & 1 \\
236 & 1 & 4 & 2 & 2 & 1 \\
236 & 1 & 3 & 3 & 2 & 1 \\
236 & 1 & 2 & 4 & 2 & 1 \\
\hline \hline
\end{tabular}

\section{A.3 rwm5yr}

Os dados do banco rwm5yr podem ser adquiridos atrvés do pacote geepack no $\mathrm{R}$, com os seguintes comandos:

install .packages ("geepack")

library (geepack)

data ("rwm5yr") 


\section{Apêndice B}

\section{Códigos do R e do SAS}

\section{B.1 Leprosy}

\section{B.1.1 R}

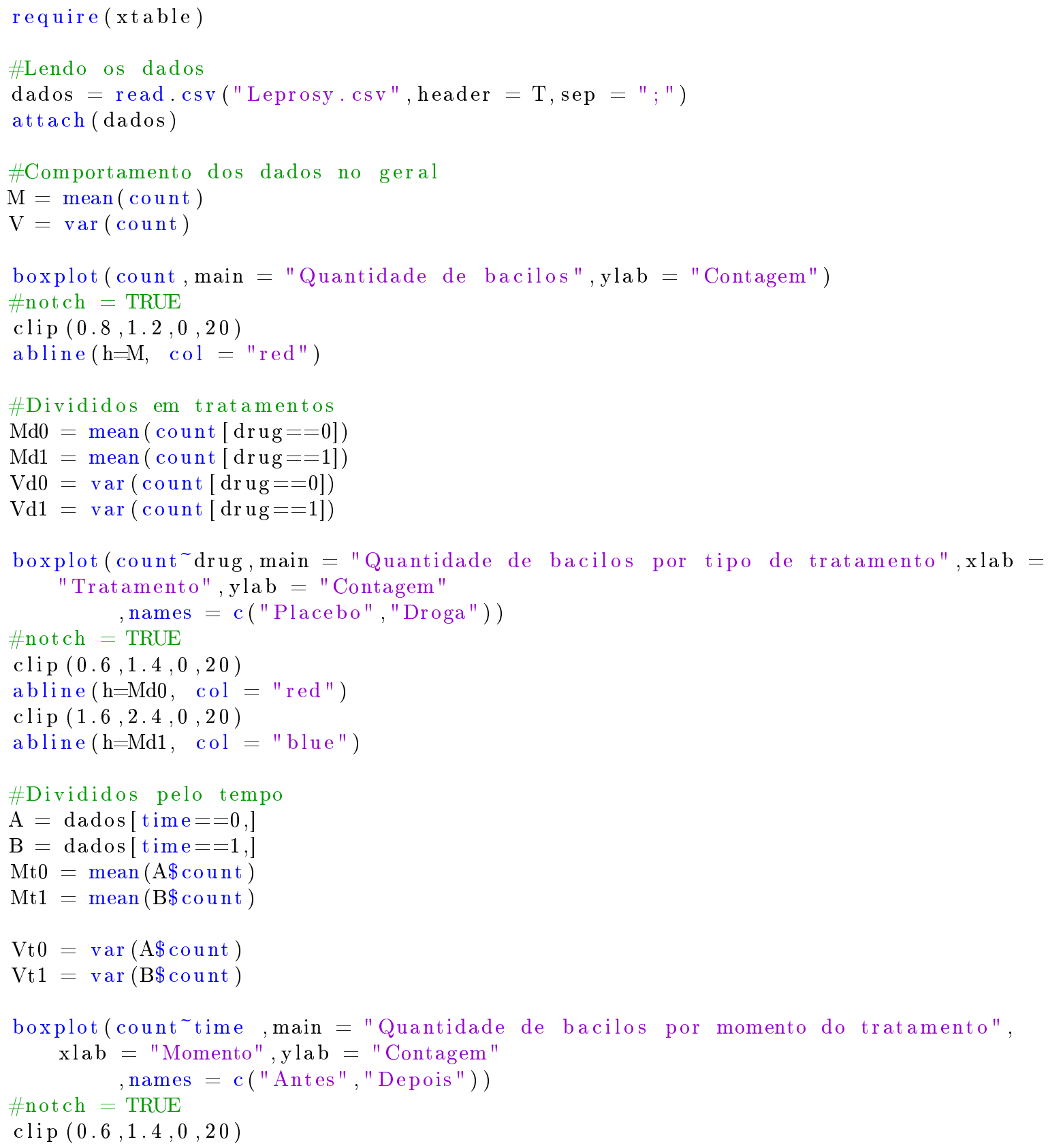




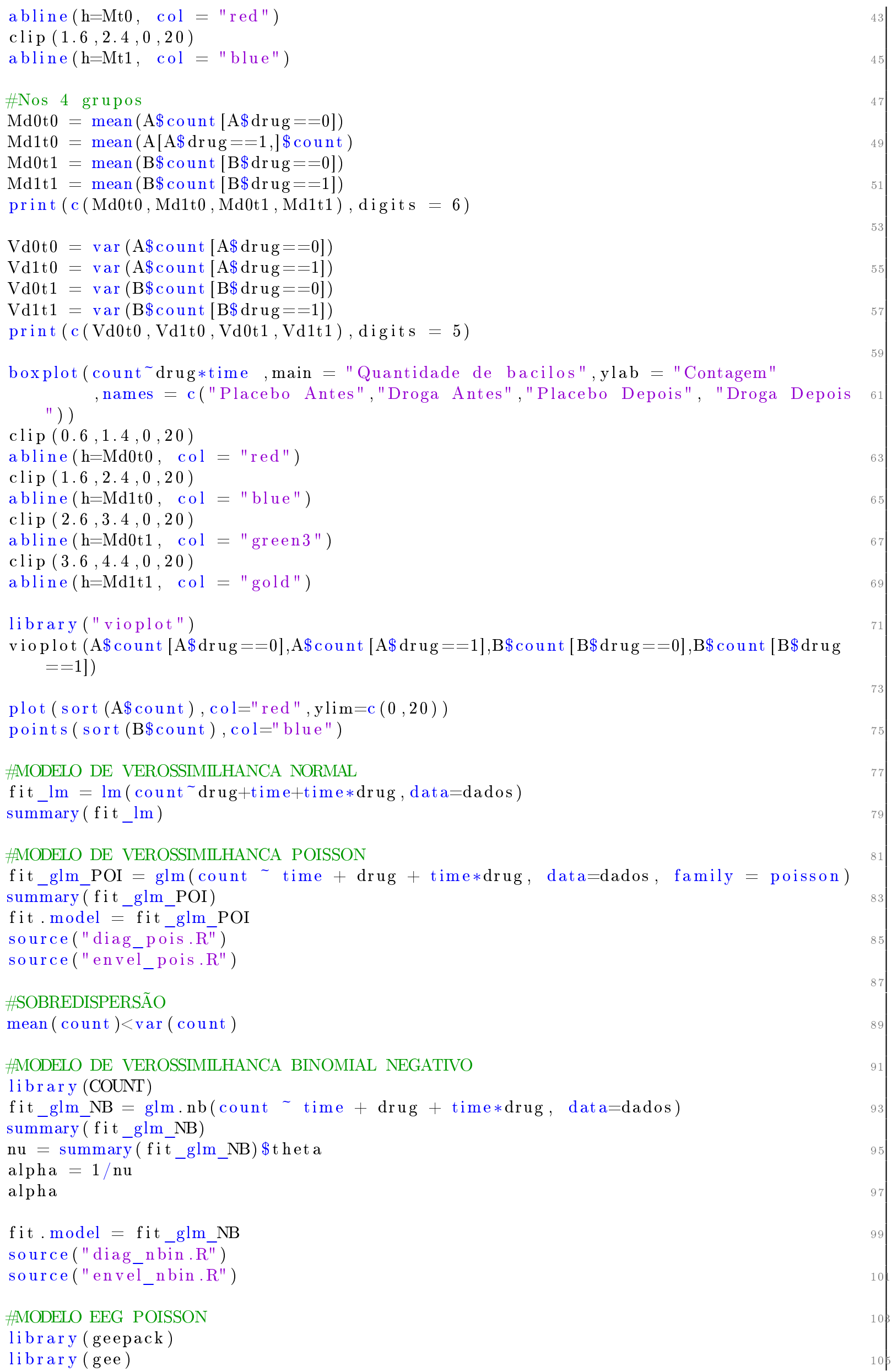


fit_gee_POI $=$ geeglm $\left(\right.$ count ${ }^{\sim}$ time+drug+time $*$ drug,, data=dados, $\quad$ id $=$ id, family $=$ poisson, corstr = "exchangeable")

summary(fit_gee_POI)

fit.model $=$ fit_gee_POI

source("diag_gee_pois.R")

source("envel gee pois.R")

diag_gee_poisson(

envelope_gee_poisson (fit.model, dados=dados)

requir e (MuMIn)

QIC (fit.model)

model.sel (fit.model, rank = QIC)

\section{\#MODELO DE COPULAS GAUSSIANAS POISSON}

require ("gcmr")

fit_cop_POI $=$ gcmr $($ count $\sim$ time+drug+time $*$ drug, data=dados, marginal $=$ poisson.marg , id $=$ id ,

cormat $=$ cluster. cormat $($ id, type="exchangeable" $))$

summary(fit_cop_POI)

\section{\#MODELO DE COPULAS GAUSSIANAS BINOMIAL NEGATIVO}

fit_cop_NB $=$ gcmr $($ count $\sim$ time + drug+time $*$ drug, data=dados, marginal = negbin.marg , $\mathrm{id}=\overline{\mathrm{id}}$,

summary (fit_cop_NB)

$$
\text { cormat }=\text { cluster.cormat }(\text { id , type="exchangeable" }))
$$

fit_cop_NB $=$ gcmr $($ count $\sim$ time+drug+time $*$ drug, data=dados, marginal $=$ negbin.marg , $\overline{\mathrm{i}} \mathrm{d}=\overline{\mathrm{id}}$,

summary (fit_cop_NB)

cormat $=$ cluster. $\operatorname{cormat}($ id, type="independence" $))$

fit_cop_NB $=$ gcmr $($ count $\sim$ time + drug+time $*$ drug, data=dados, marginal $=$ negbin.marg , $\mathrm{id}=\overline{\mathrm{id}}$,

$\operatorname{summary}(\mathrm{f}$ it_cop_NB)

cormat $=$ cluster. $\operatorname{cormat}($ id, type $=" \operatorname{ar} 1 "))$

fit_cop_NB $=$ gcmr $($ count $\sim$ time + drug+time $*$ drug, data=dados, marginal $=$ negbin.marg , $\mathrm{i} d=\overline{\mathrm{id}}$,

summary (fit_cop_NB)

cormat $=$ cluster. cormat $($ id, type $=$ "unstructured" $))$

fit_cop_NB $=$ gcmr $($ count $\sim$ time+drug, data=dados, marginal $=$ negbin.marg, id $=$ id , cormat $=$ cluster. $\operatorname{cormat}(\mathrm{id}$, type $=$ "exchangeable" $)$ )

$\operatorname{summary}\left(\mathrm{fit} \_\right.$cop_NB)

fit_cop_NB2 $=$ gcmr $($ count $\sim$ time, data=dados, marginal $=$ negbin.marg, id $=$ id ,

summary (fit_cop_NB2) cormat $=$ cluster. $\operatorname{cormat}($ id, type $=$ "exchangeable" $)$ )

\section{B.1.2 SAS}

PROC GENMOD DATA $=$ WORK. LEPROSY

PLOTS $($ ONLY $)=$ ALL;

CLASS ID id;

MODEL COUNT $=$ TIME DRUG TIME $*$ DRUG $/$ LINK=LOG DIst=p oisson TYPE3; REPEATED SUBJECT=ID / SORTED WTTHINSUBJECT=id TYPE-EXCH COVB CORRW MODELSE; RUN; 
PROC GENMOD DATA-WORK. LEPROSY

PLOTS $($ ONLY $)=$ ALL;

CLASS ID id;

MODEL COUNT $=$ TIME DRUG TIME $*$ DRUG $/$ LINK=LOG DISt=negbin TYPE3;

REPEATED SUBJECT=ID / SORTED WITHINSUBJECT=id TYPE-EXCH COVB CORRW MODELSE;

RUN;

PROC GENMOD DATA=WORK.LEPROSY

PLOTS $($ ONLY $)=$ ALL;

CLASS ID id;

MODEL COUNT $=$ TIME DRUG TIME $*$ DRUG $/$ LINK=LOG DISt $=$ neg bin TYPE3;

REPEATED SUBJECT=ID / SORTED WITHINSUBJECT=id TYPE=AR COVB CORRW MODELSE;

RUN;

PROC GENMOD DATA-WORK. LEPROSY

$\operatorname{PLOTS}(\mathrm{ONLY})=\mathrm{ALL}$;

CLASS ID id;

MODEL COUNT = TIME DRUG TIME $*$ DRUG / LINK=LOG DISt=negbin TYPE3;

REPEATED SUBJECT=ID / SORTED WITHINSUBJECT $=\mathrm{i}$ d TYPE=IND COVB CORRW MODELSE;

RUN;

PROC GENMOD DATA=WORK. LEPROSY

$\operatorname{PLOTS}(\mathrm{ONLY})=\mathrm{ALL}$;

CLASS ID id;

MODEL COUNT $=$ TIME DRUG TIME $*$ DRUG $/$ LINK=LOG DISt $=$ neg bin TYPE3;

REPEATED SUBJECT=ID / SORTED WTTHINSUBJECT=id TYPE-UNSTR COVB OORRW MODELSE;

RUN;

PROC GENMOD DATA=WORK.LEPROSY

PLOTS $($ ONLY $)=$ ALL;

CLASS ID id;

MODEL COUNT $=$ TIME $/$ LINK=LOG DISt=negbin TYPE3;

REPEATED SUBJECT=ID / SORTED WITHINSUBJECT=id TYPE-EXCH COVB CORRW MODELSE; RUN;

\section{B.2 Progabide}

\section{B.2.1 R}

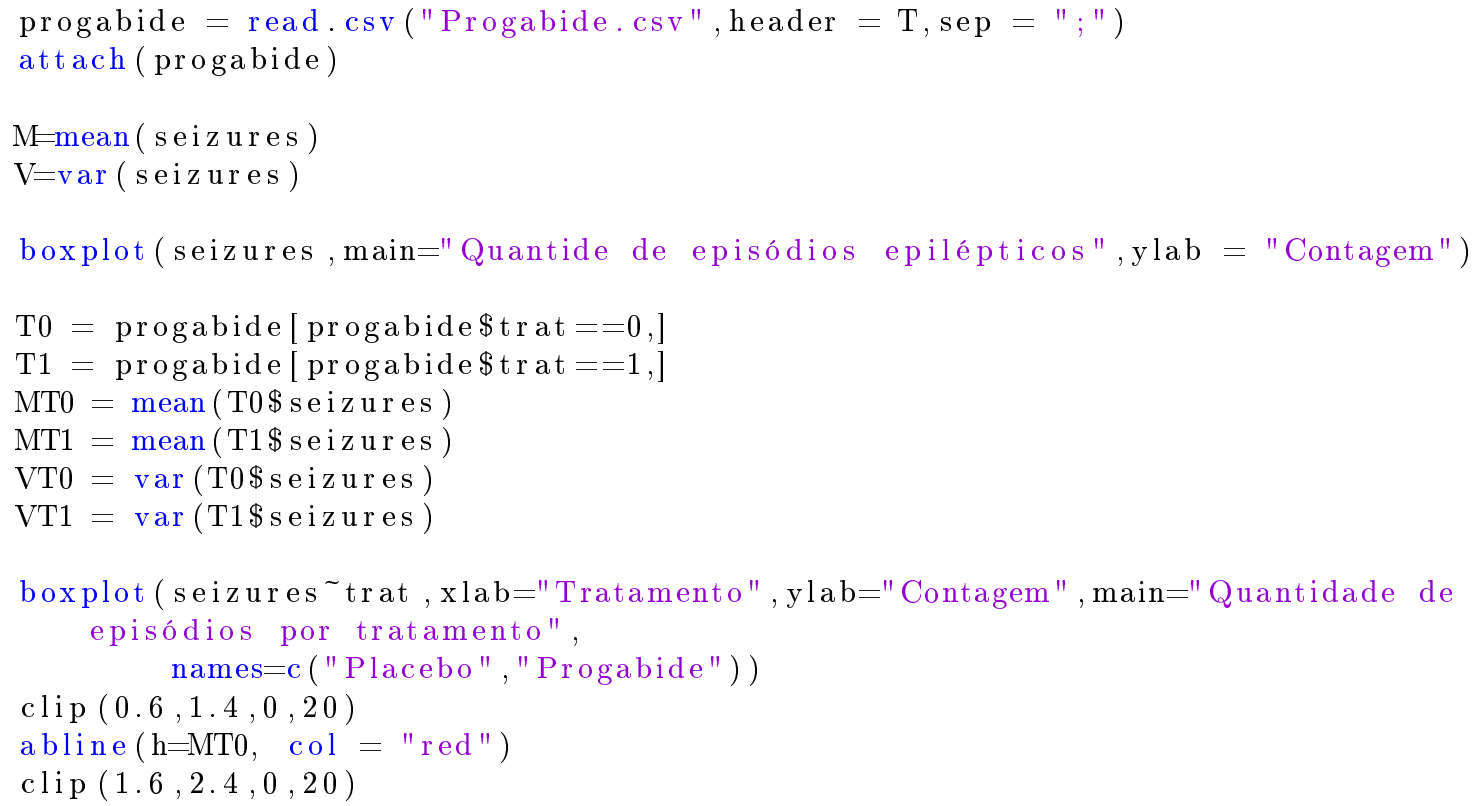


abline (h=MT1, col = "blue")

plot $(\mathrm{T} 0 \$ \mathrm{t}, \log (\mathrm{T} 0 \$ \mathrm{seizures})$, type $=" 1 ")$

points $(\mathrm{T} 1 \$ \mathrm{t}, \log (\mathrm{T} 1 \$ \mathrm{seizures})$, type $=" 1 ", \mathrm{col}=$ "blue")

$\mathrm{Mt} 0=$ mean $($ progabide $[$ progabide $\$ \mathrm{t}==0] \$,$$ seizures )$

$\mathrm{Mt} 1=$ mean $($ progabide [ progabide $\$ \mathrm{t}==1, \mid \$ \mathrm{seizures}$ )

Mt $2=$ mean $($ progabide $[$ progabide $\$ \mathrm{t}==2,1 \$$ seizures $)$

Mt $3=$ mean (progabide [ progabide $\$ \mathrm{t}==3] \$,$$ seizures )$

Mt $4=$ mean (progabide $[$ progabide $\$ \mathrm{t}==4] \$,$$ seizures )$

Vt $0=\operatorname{var}($ progabide [ progabide $\$ \mathrm{t}==0] \$,$$ seizures )$

$\mathrm{Vt} 1=\operatorname{var}($ progabide [ progabide $\$ \mathrm{t}==1] \$,$$ seizures )$

$\mathrm{Vt} 2=\operatorname{var}($ progabide $[$ progabide $\$ \mathrm{t}==2] \$,$$ seizures )$

Vt $3=\operatorname{var}($ progabide $[$ progabide $\$ \mathrm{t}==3] \$,$$ seizures )$

$\mathrm{Vt} 4=\operatorname{var}($ progabide $[$ progabide $\$ \mathrm{t}==4] \$,$$ seizures )$

Medias $=\mathrm{c}(\mathrm{Mt0}, \mathrm{Mt} 1, \mathrm{Mt} 2, \mathrm{Mt} 3, \mathrm{Mt} 4)$

Momentos $=1: 5$

plot (Momentos, Medias, type $=" b ")$

boxplot (seizures〜t, main="Quantidade de episódios por momento", xlab="Momento", ylab="Contagem", names=c ( "Base" , "1", "2" , "3" , "4") )

clip $(0.6,1.4,0,150)$

abline $(\mathrm{h}=\mathrm{Mt0}, \quad \mathrm{col}=$ "red")

clip $(1.6,2.4,0,150)$

abline $(\mathrm{h}=\mathrm{Mt1}$, col = "blue")

clip $(2.6,3.4,0,150)$

abline $(\mathrm{h}=\mathrm{Mt} 2, \operatorname{col}=$ "green $3 ")$

clip $(3.6,4.4,0,150)$

abline $(\mathrm{h}=\mathrm{Mt} 3$, col $=$ "gold" $)$

clip $(4.6,5.4,0,150)$

abline $(\mathrm{h}=$ Mt4, col = "purple")

M0t0 $=\operatorname{mean}(\mathrm{T} 0[\mathrm{~T} 0 \$ \mathrm{t}=0] \,$ \mathrm{seizures})$

$\mathrm{M} 0 \mathrm{t} 1=\operatorname{mean}(\mathrm{T} 0[\mathrm{~T} 0 \$ \mathrm{t}==1,1 \$ \mathrm{seizures})$

M0t2 $=\operatorname{mean}(\mathrm{T} 0[\mathrm{~T} 0 \$ \mathrm{t}==2] \$,$$ seizures )$

$\mathrm{M} 0 \mathrm{t} 3=\operatorname{mean}(\mathrm{T} 0[\mathrm{~T} 0 \$ \mathrm{t}==3] \,$ \mathrm{seizures})$

$\mathrm{M} 0 \mathrm{t} 4=\operatorname{mean}(\mathrm{T} 0[\mathrm{~T} 0 \$ \mathrm{t}==4] \,$ \mathrm{seizures})$

$\mathrm{M} 1 \mathrm{t} 0=\operatorname{mean}(\mathrm{T} 1[\mathrm{~T} 0 \$ \mathrm{t}==0] \,$ \mathrm{seizures})$

$\mathrm{M} 1 \mathrm{t} 1=\operatorname{mean}(\mathrm{T} 1[\mathrm{~T} 0 \$ \mathrm{t}==1, \mid \$$ seizures $)$

M1t2 $=\operatorname{mean}(\mathrm{T} 1[\mathrm{~T} 0 \$ \mathrm{t}==2, \mid \$$ seizures $)$

M1t3 $=\operatorname{mean}(\mathrm{T} 1[\mathrm{~T} 0 \$ \mathrm{t}==3] \$,$$ seizures )$

$\mathrm{M} 1 \mathrm{t} 4=\operatorname{mean}(\mathrm{T} 1[\mathrm{~T} 0 \$ \mathrm{t}==4] \,$ \mathrm{seizures})$

V0t0 $=\operatorname{var}(\mathrm{T} 0[\mathrm{~T} 0 \$ \mathrm{t}==0] \$,$$ seizures )$

$\mathrm{V} 0 \mathrm{t} 1=\operatorname{var}(\mathrm{T} 0[\mathrm{~T} 0 \$ \mathrm{t}==1] \$,$$ seizures )$

V0t2 $=\operatorname{var}(\mathrm{T} 0[\mathrm{~T} 0 \$ \mathrm{t}==2] \$,$$ seizures )$

$\operatorname{Vot} 3=\operatorname{var}(\mathrm{T} 0[\mathrm{~T} 0 \$ \mathrm{t}==3,1 \$$ seizures $)$

V0t4 $=\operatorname{var}(\mathrm{T} 0[\mathrm{~T} 0 \$ \mathrm{t}==4] \$,$$ seizures )$

$\mathrm{V} 1 \mathrm{t} 0=\operatorname{var}(\mathrm{T} 1[\mathrm{~T} 0 \$ \mathrm{t}==0$,$] seizures )$

$\mathrm{V} 1 \mathrm{t} 1=\operatorname{var}(\mathrm{T} 1[\mathrm{~T} 0 \$ \mathrm{t}==1] \$,$$ seizures )$

$\mathrm{V} 1 \mathrm{t} 2=\operatorname{var}(\mathrm{T} 1[\mathrm{~T} 0 \$ \mathrm{t}==2, \$$ seizures $)$

$\mathrm{V} 1 \mathrm{t} 3=\operatorname{var}(\mathrm{T} 1[\mathrm{~T} 0 \$ \mathrm{t}==3] \$,$$ seizures )$

$\mathrm{V} 1 \mathrm{t} 4=\operatorname{var}(\mathrm{T} 1[\mathrm{~T} 0 \$ \mathrm{t}==4] \$,$$ seizures )$

$\mathrm{M} 0=\mathrm{c}(\mathrm{M} 0 \mathrm{t} 1, \mathrm{M} 0 \mathrm{t} 2, \mathrm{M} 0 \mathrm{t} 3, \mathrm{M} 0 \mathrm{t} 4)$

$\mathrm{M} 1=\mathrm{c}(\mathrm{M} 1 \mathrm{t} 1, \mathrm{M} 1 \mathrm{t} 2, \mathrm{M} 1 \mathrm{t} 3, \mathrm{M} 1 \mathrm{t} 4)$

$\mathrm{V} 0=\mathrm{c}(\mathrm{V} 0 \mathrm{t} 0, \mathrm{~V} 0 \mathrm{t} 1, \mathrm{~V} 0 \mathrm{t} 2, \mathrm{~V} 0 \mathrm{t} 3, \mathrm{~V} 0 \mathrm{t} 4)$

$\mathrm{V} 1=\mathrm{c}(\mathrm{V} 1 \mathrm{t} 0, \mathrm{~V} 1 \mathrm{t} 1, \mathrm{~V} 1 \mathrm{t} 2, \mathrm{~V} 1 \mathrm{t} 3, \mathrm{~V} 1 \mathrm{t} 4)$

plot $(\log (\mathrm{M} 0)$, type $=" 1 "$, ylim $=\mathrm{c}(1.8,2.3)$, frame $\cdot$ plot $=\mathrm{T}$, axes $=\mathrm{F})$ 


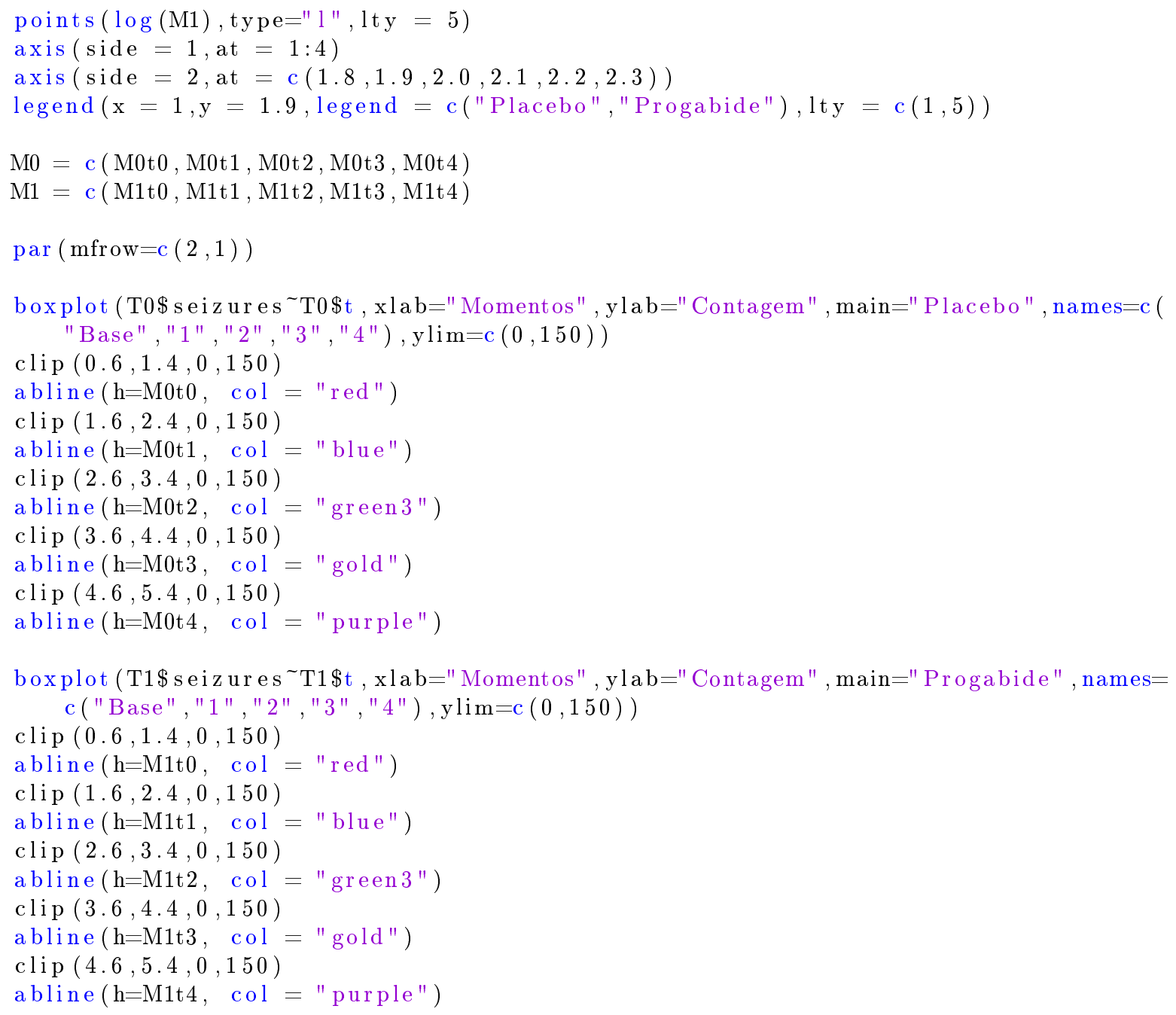

\#MODELO DE VEROSSIMILHANCA NORMAL

fit_lm $=\operatorname{lm}($ seizures $\sim$ trat + tempo + trat $*$ tempo, offset $=\log (\mathrm{m})$, data=progabide $)$ summary (fit_lm)

\#MODELO DE VEROSSTMILHANCA POISSON

fit_glm_POI $=$ glm $($ seizures $\sim$ trat +tempo +trat $*$ tempo, offset $=\log (\mathrm{m})$, data $=$ progabide, family $=$ poisson $)$

summary (fit_glm_POI)

fit.model =fit_glm_POI

source ("diag_pois.R")

source ("envel_pois.R")

\#MODELO DE VEROSSIMILHANCA BINOMIAL NEGATIVO

library (COUNT)

fit_glm_NB $=$ glm.nb( seizures ${ }^{\sim}$ offset $(\log (\mathrm{m}))+$ trat +tempo +trat $*$ tempo, data $=$ progabide)

summary(fit_glm_NB)

$\mathrm{nu}=\operatorname{summary}\left(\mathrm{fit} \_\mathrm{glm} \_\mathrm{NB}\right) \$$ theta

alpha $=1 / \mathrm{nu}$

alpha

fit.model $=$ fit_glm_NB

source ("diag nbin.R")

source ("envel_nbin.R")

\#MODELO EEG POISSON

library (geepack)

library (gee) 
fit gee POI $=$ gee $($ seizures $\sim$ offset $(\log (\mathrm{m}))+$ trat +tempo + trat $*$ tempo, data $=$ progabide, corstr $=$ "exchangeable",id $=$ id,family $=$ "poisson") summary(fit_gee_POI)

fit.model $=$ fit_gee_POI

source("diag_gee_pois.R")

source("envel_gee_pois.R")

analise_diag_poisson $<-$ diag_gee_poisson(fit.model, progabide, umaJanela=T, sel $\overline{\mathrm{G}} \mathrm{raficos}=\mathrm{c}(0,0,1,1)$, identific $=\mathrm{c}(0,0,1,1))$

$\operatorname{par}(\operatorname{mfrow}=\mathrm{c}(1,1))$

envelope_gee_poisson(fit.model, progabide, analise_diag_poisson, opcaoEnvelope=" Normal")

r equir e (MuMIn)

QIC (fit.model)

model.sel (fit.model, rank = QIC)

\section{\#MODELO DE COPULAS GAUSSIANAS POISSON}

require ( "gcmr")

fit_cop_POI $=$ gcmr $($ seizures $\sim$ offset $(\log (\mathrm{m}))+$ trat +tempo +trat $*$ tempo, data $=$ progabide,

summary(fit_cop_POI)

marginal $=$ poisson.marg, $\mathrm{id}=\mathrm{ID}$,

cormat $=$ cluster. cormat $($ ID, type $=$ "exchangeable" $)$ )

xtable (summary (fit_cop_POI) )

\#MODELIO DE COPULAS GAUSSTANAS BINOMIAL NEGATIVO

fit_cop_NB $=$ gcmr $($ seizures $\sim$ offset $(\log (\mathrm{m}))+$ trat +tempo +trat $*$ tempo, data=progabide summary (fit_cop_NB)

marginal $=$ negbin $\cdot \operatorname{marg}, \mathrm{id}=\mathrm{ID}$,

cormat $=$ cluster. $\operatorname{cormat}($ ID, type $=$ "exchangeable" $))$

fit_cop_NB $=$ gcmr $($ seizures $\sim$ offset $(\log (\mathrm{m}))+$ trat +tempo +trat $*$ tempo, data=progabide , marginal $=$ negbin $. \operatorname{marg}, \mathrm{id}=\mathrm{ID}$,

cormat $=$ cluster. cormat $($ ID , type="exchangeable" $)$, subset $=-c$

$(241,242,243,244,245))$

summary (fit_cop_NB)

fit_cop_NB $=$ gcmr $($ seizures offset $(\log (\mathrm{m}))+$ trat +tempo, data=progabide , marginal $=$ negbin. marg, $\mathrm{id}=\mathrm{ID}$,

cormat $=$ cluster . cormat (ID, type="exchangeable"), subset $=-c$

$(241,242,243,244,245))$

summary (fit_cop_NB)

fit_cop_NB $=$ gcmr $($ seizures $\sim$ offset $(\log (m))+$ trat, data=progabide

, marginal $=$ negbin.marg, $\mathrm{id}=\mathrm{ID}$,

cormat $=$ cluster. cormat $($ ID, type $="$ exchangeable" $)$, subset $=-c$

$(241,242,243,244,245))$

summary (fit_cop_NB)

fit_cop_NB $=$ gcmr $($ seizures $\sim$ offset $(\log (\mathrm{m}))+$ tempo, data=progabide

, marginal $=$ negbin. marg, $\mathrm{id}=\mathrm{ID}$,

cormat $=$ cluster . cormat (ID, type="exchangeable"), subset $=-c$

$(241,242,243,244,245))$

summary(fit_cop_NB)

fit_cop_NB $=$ gcmr $($ seizures $\sim$ offset $(\log (\mathrm{m}))$, data=progabide

, marginal $=$ negbin.marg, $\mathrm{id}=\mathrm{ID}$,

cormat $=$ cluster. cormat $($ ID , type="exchangeable" $)$, subset $=-c$

$(241,242,243,244,245))$

summary (fit_cop_NB) 


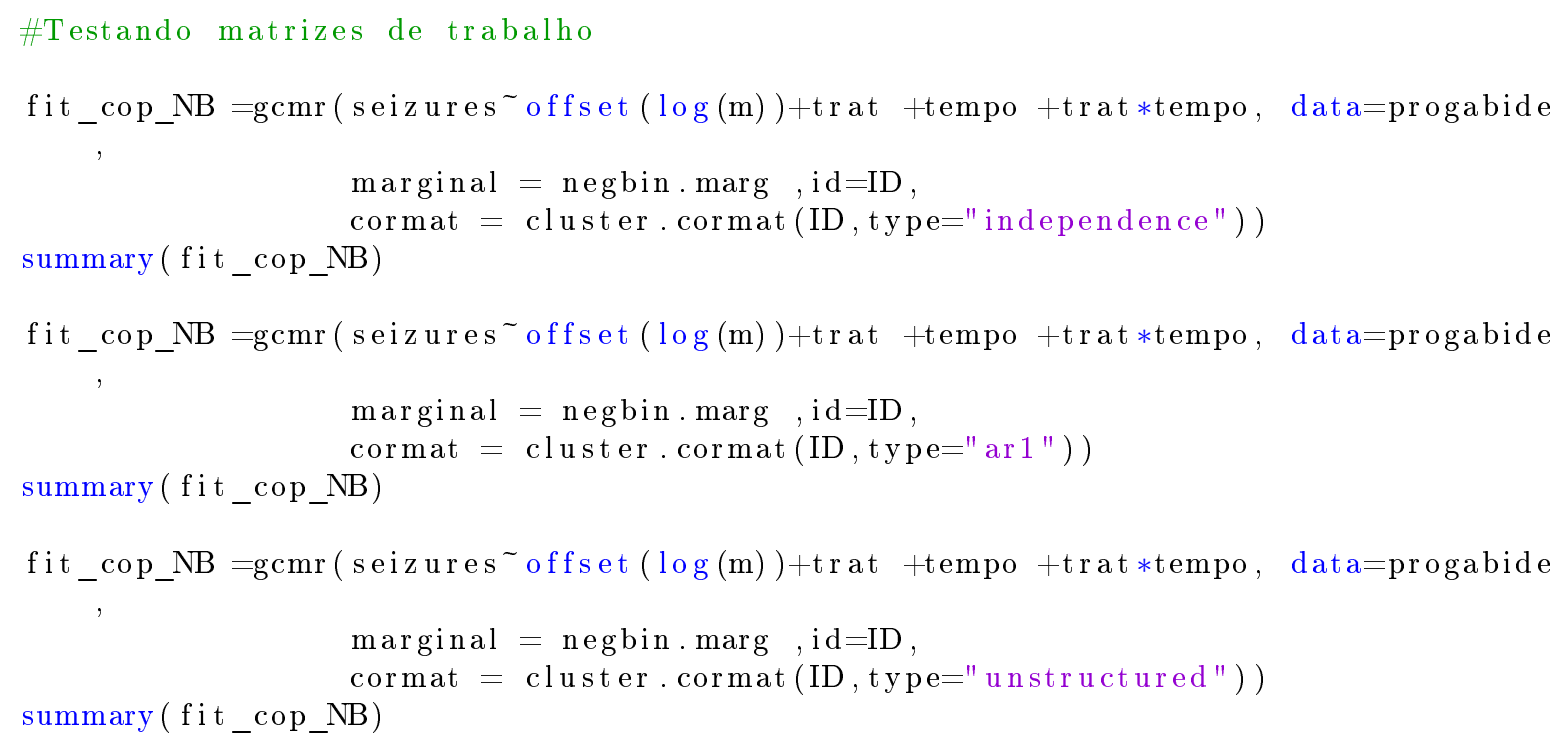

\section{B.2.2 SAS}

PROC GENMOD DATA-WORK.QUERY_FOR_PROGABIDE

$\operatorname{PLOTS}(\mathrm{ONLY})=\mathrm{ALL}$;

CLASS ID id;

MODEL SEIZURES = TRAT TEMPO TEMPO $*$ TRAT $/$ LINK=LOG DIST=POISSON TYPE3 OFFSET= LOGM;

REPEATED SUBJECT=ID / SORTED WITHINSUBJECT=id TYPE-EXCH COVB CORRW MODELSE;

RUN;

PROC GENMOD DATA=WORK.QUERY_FOR_PROGABIDE

PLOTS $($ ONLY $)=$ ALL;

CLASS ID id;

MODEL SEIZURES $=$ TRAT TEMPO TEMPO $*$ TRAT $/$ LINK=LOG DISt $=$ neg bin TYPE3 OFFSET $=$ LOGM

REPEATED SUBJECT=ID / SORTED WITHINSUBJECT=id TYPE=IND COVB OORRW MODELSE;

RUN;

PROC GENMOD DATA=WORK.QUERY_FOR_PROGABDE

$\operatorname{PLOTS}(\mathrm{ONLY})=\mathrm{ALL}$;

CLASS ID id;

MODEL SEIZURES $=$ TRAT TEMPO TEMPO $*$ TRAT $/$ LINK=LOG DISt $=$ neg bin TYPE3 OFFSET $=$ LOGM REPEATED SUBJECT=ID / SORTED WITHINSUBJECT=id TYPE-AR COVB CORRW MODELSE;

RUN;

PROC GENMOD DATA-WORK.QUERY_FOR_PROGABIDE

$\operatorname{PLOTS}(\mathrm{ONLY})=\mathrm{ALL}$;

CLASS ID id:

MODEL SEIZURES $=$ TRAT TEMPO TEMPO $*$ TRAT $/$ LINK=LOG DISt $=$ neg bin TYPE3 OFFSET= LOGM REPEATED SUBJECT=ID / SORTED WTTHINSUBJECT=id TYPe= unstr COVB CORRW MODELSE;

RUN;

PROC GENMOD DATA WORK. QUERY FOR PROGABIDE

PLOTS $($ ONLY $)=$ ALL;

CLASS ID id;

MODEL SEIZURES $=$ TRAT TEMPO TEMPO $*$ TRAT $/$ LINK=LOG DISt $=$ neg bin TYPE3 OFFSET $=$ LOGM

REPEATED SUBJECT=ID / SORTED WITHINSUBJECT=id TYPE-EXCH COVB CORRW MODELSE;

RUN;

PROC GENMOD DATA=WORK.QUERY_FOR_PROGABIDE

$\operatorname{PLOTS}(\mathrm{ONLY})=\mathrm{ALL}$ 
CLASS ID id;

MODEL SEIZURES = TRAT TEMPO / LINK=LOG DISt=negbin TYPE3 OFFSET= LOGM; REPEATED SUBJECT=ID / SORTED WITHINSUBJECT=id TYPE-EXCH COVB CORRW MODELSE; RUN;

PROC GENMOD DATA=WORK. QUERY FOR PROGABIDE

PLOTS $($ ONLY $)=$ ALL;

CLASS ID id;

MODEL SEIZURES $=$ TRAT $/$ LINK=LOG DISt=negbin TYPE3 OFFSET= LOGM;

REPEATED SUBJECT=ID / SORTED WITHINSUBJECT=id TYPE-EXCH COVB CORRW MODELSE;

RUN;

PROC GENMOD DATA $=$ WORK. QUERY FOR PROGABIDE

$\operatorname{PLOTS}(\mathrm{ONLY})=\mathrm{ALL}$;

CLASS ID id;

MODEL SEIZURES $=$ TEMPO / LINK $=$ LOG DISt=neg bin TYPE3 OFFSET= LOGM;

REPEATED SUBJECT=ID / SORTED WITHINSUBJECT=id TYPE-EXCH COVB CORRW MODELSE;

RUN;

PROC GENMOD DATA-WORK. QUERY FOR PROGABIDE

$\operatorname{PLOTS}(\mathrm{ONLY})=$ ALL;

CLASS ID id;

MODEL SEIZURES $=/$ LINK=LOG DISt=neg bin TYPE3 OFFSET $=$ LOGM;

REPEATED SUBJECT=ID / SORTED WITHINSUBJECT=id TYPE-EXCH COVB CORRW MODELSE; RUN;

\section{B.3 rwm5yr}

\section{B.3.1 R}

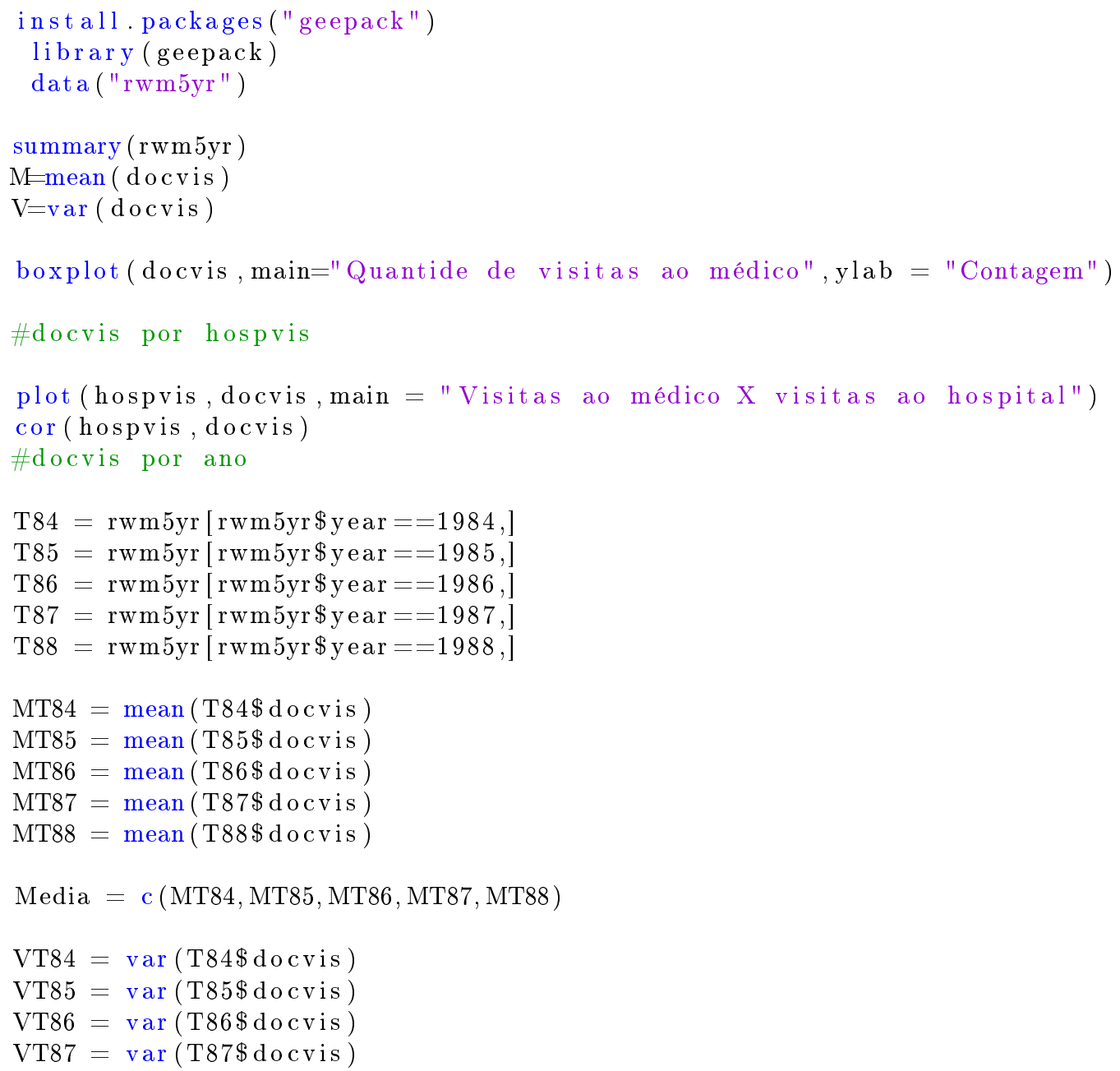




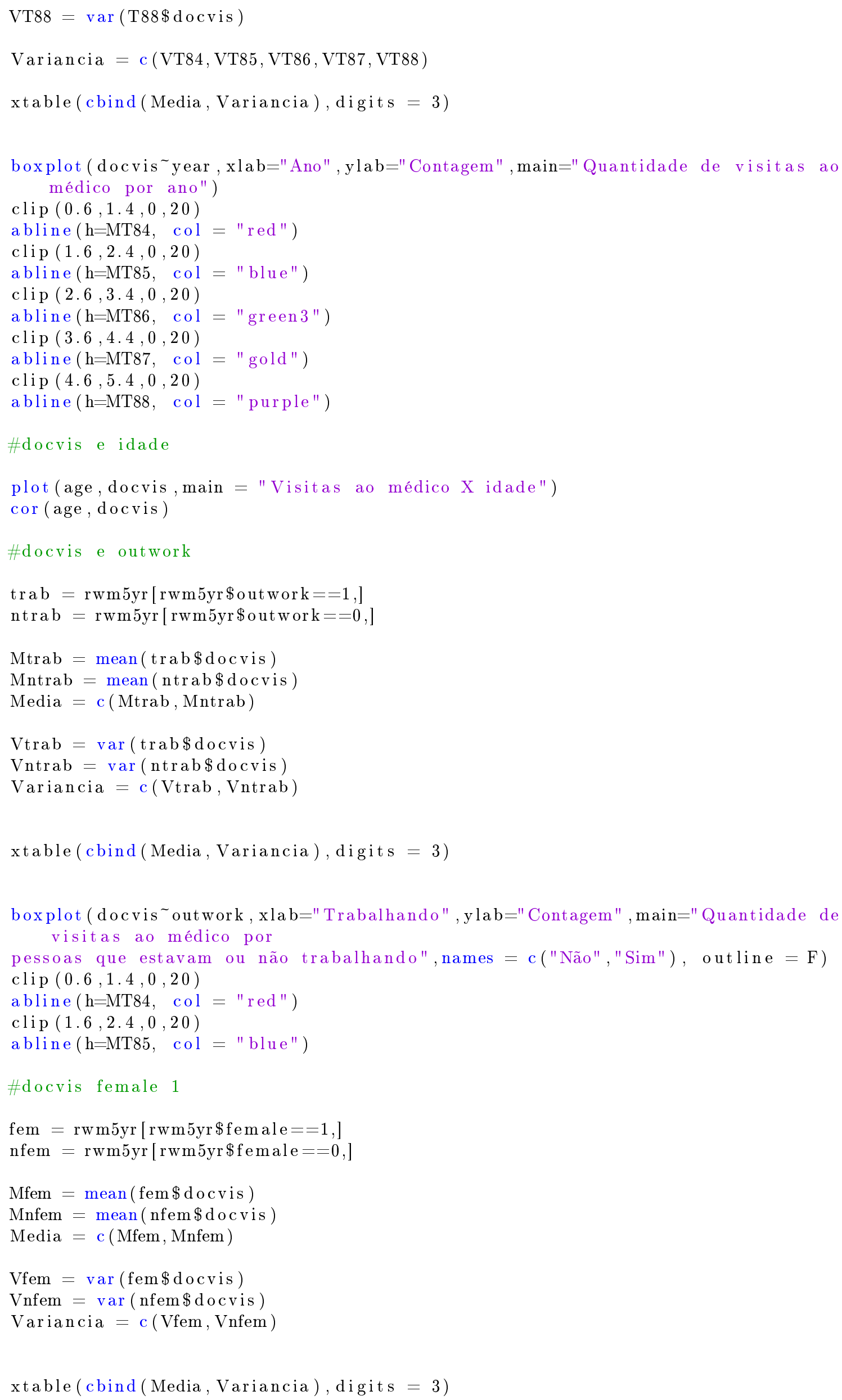


boxplot (docvis female, $x l a b="$ Gênero",ylab="Contagem", main="Quantidade de visitas ao médico por gênero", names = c("Masculino","Feminino"))

clip $(0.6,1.4,0,20)$

abline $(\mathrm{h}=\mathrm{MT} 84, \operatorname{col}=$ "red")

clip $(1.6,2.4,0,20)$

abline $(\mathrm{h}=\mathrm{MT} 85$, col $=$ "blue")

boxplot (docvis female, $x l a b="$ Gênero",ylab="Contagem", main="Quantidade de visitas ao médico por gênero", names = c("Masculino", "Feminino"), outline=F)

clip $(0.6,1.4,0,20)$

abline $(\mathrm{h}=\mathrm{MT} 84, \mathrm{col}=$ "red")

clip $(1.6,2.4,0,20)$

abline $(\mathrm{h}=\mathrm{MT} 85$, col $=$ "blue" $)$

\#docvis edlevel 1 a 4

ed $1=\operatorname{rwm} 5 y r[\operatorname{rwm} 5 y r \$$ edlevel $1==1$,

$\operatorname{ed} 2=\operatorname{rwm} 5 y r[\operatorname{rwm} 5 y r \$ \operatorname{edlevel} 2==1$,

ed3 $=\operatorname{rwm} 5 y r[\operatorname{rwm} 5 y r \$ \operatorname{edlevel} 3==1$,

$\operatorname{ed} 4=\operatorname{rwm} 5 y r[\operatorname{rwm} 5 y r \$$ edlevel $4==1$,

Med1 $=\operatorname{mean}(\operatorname{ed} 1 \$$ docvis $)$

$\operatorname{Med} 2=\operatorname{mean}(\operatorname{ed} 2 \$$ docvis $)$

Med3 $=\operatorname{mean}(\operatorname{ed} 3 \$$ docvis $)$

$\operatorname{Med} 4=\operatorname{mean}(\operatorname{ed} 4 \$$ docvis $)$

Media $=\mathrm{c}(\operatorname{Med} 1, \operatorname{Med} 2, \operatorname{Med} 3, \operatorname{Med} 4)$

Ved1 $=\operatorname{var}(\operatorname{ed} 1 \$$ docvis $)$

$\operatorname{Ved} 2=\operatorname{var}(\operatorname{ed} 2 \$$ docvis $)$

Ved3 = var (ed3\$docvis $)$

$\operatorname{Ved} 4=\operatorname{var}($ ed $4 \$$ docvis $)$

Variancia $=\mathrm{c}(\operatorname{Ved} 1, \operatorname{Ved} 2, \operatorname{Ved} 3, \operatorname{Ved} 4)$

xtable( cbind( Media, Variancia), digits $=3$ )

boxplot (docvis edlevel, xlab="Nível de educação", ylab="Contagem",main="Quantidade de visitas ao médico por nível de educação")

clip $(0.6,1.4,0,20)$

abline $(\mathrm{h}=\mathrm{MT} 84, \mathrm{col}=$ "red")

clip $(1.6,2.4,0,20)$

abline $(\mathrm{h}=$ MT85, col $=$ "blue" $)$

clip $(2.6,3.4,0,20)$

abline $(\mathrm{h}=$ MT86, col = "green $3 ")$

clip $(3.6,4.4,0,20)$

abline $(\mathrm{h}=$ MT87, $\operatorname{col}=$ "gold")

boxplot (docvis edlevel, xlab="Nível de educação", ylab="Contagem", main="Quantidade de visitas ao médico por nível de educação", outline=F)

clip $(0.6,1.4,0,20)$

abline $(\mathrm{h}=\mathrm{MT} 84, \mathrm{col}=$ "red")

clip $(1.6,2.4,0,20)$

abline $(\mathrm{h}=$ MT85, $\operatorname{col}=$ "blue")

clip $(2.6,3.4,0,20)$

abline $(\mathrm{h}=$ MT $86, \quad$ col $=$ "green $3 ")$

clip $(3.6,4.4,0,20)$ 


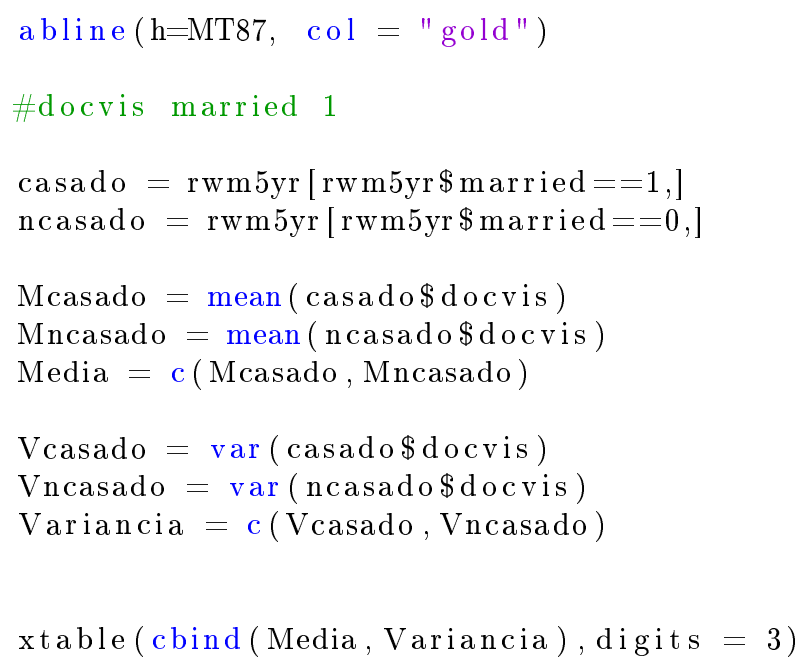


abline $(\mathrm{h}=\mathrm{MT} 85$, col $=$ "blue")

\#MODELO DE VEROSSIMLHANCA NORMAL

fit_lm $=\operatorname{lm}($ docvis $\sim$ hospvis + age+outwork + female+as.factor $($ edlevel $)+$ married + kids data $=$ rwm5yr)

summary (fit_lm)

xtable( summary(fit_lm), digits $=3$ )

\#MODELO DE VEROSSIMLHANCA POISSON

fit_glm_POI $=$ glm $($ docvis $\sim$ hospvis + age+outwork + female + as. factor $($ edlevel $)+$ married + kids,

summary(fit_glm_POI)

$$
\text { data }=\text { rwm5yr, family }=\text { poisson })
$$

xtable(summary ( $\bar{f}$ it_glm_POI), digits $=3$ )

fit.model $=$ fit_glm_POI

source ("diag_pois. $\mathrm{R}^{\overline{1}}$ )

\#source ("envel_pois.R")

\section{\#MODELO DE VEROSSIMILHANCA BINOMIAL NEGATIVO}

library (COUNT)

fit_glm_NB $=$ glm.nb(docvis $\sim$ hospvis+age+outwork+female+as.factor $($ edlevel $)+$ married $+\mathrm{kids}$,

summary (fit_glm_NB) dat $\mathrm{a}=\mathrm{rwm} 5 \mathrm{yr})$

xtable (summary $(\bar{f}$ it_glm_NB), digits $=3$ )

$\mathrm{nu}=\operatorname{summary}(\mathrm{fit}$ glm_NB$) \$$ theta

alpha $=1 / \mathrm{nu}$

alpha

fit.model $=$ fit_glm_NB

\#source ("diag_nbin. $\overline{\mathrm{R}} "$ )

\#source ("envel_nbin.R")

fit_glm_NB $=$ glm.nb(docvis $\sim$ hospvis +age+outwork+female+as.factor (edlevel $)+$ married $+\mathrm{ki} \overline{\mathrm{ds}}$,

$\operatorname{summary}\left(\mathrm{fit} \_\mathrm{glm} \_\mathrm{NB}\right)$

$$
\text { data }=\text { rwm5yr, maxit }=50)
$$

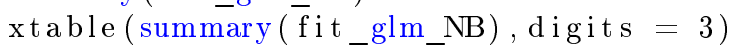

\#MODELIO EEG POISSON

library (geepack)

library (gee)

fit_gee_POI $=$ gee $($ docvis $\sim$ hospvis + age+outwork + female+as.factor $($ edlevel $)+$ married + kids poisson") , data $=$ rwm5yr, corstr $=$ "exchangeable", id $=$ id, family $="$

fit_gee_POI $=$ geeglm $($ docvis $\sim$ hospvis + as.factor $($ year $)+$ age + outwork + female+as.factor $($ ed $\bar{l}$ evel $)+$ married + kids poisson") , data $=$ rwm5yr, corstr $=$ "independence", id = id,family $=$ "

summary (fit_gee_POI)

xtable(summary( $\bar{f}$ it_gee_POI), digits=3)

fit.model $=$ fit_gee_POI

source("diag_gee_pois.R")

source("envel_gee_pois.R")

diag_gee_poisson( fit.model, dados = dados $)$

envelope_gee_poisson (fit.model, dados=dados)

\#MODEIO DE COPULAS GAUSSIANAS POISSON

require ( $\mathrm{gcmr}$ ")

$\mathrm{t} 1=$ Sys.time () 
fit cop POI $=$ gcmr (docvis $\sim$ hospvis + age + outwork + female+as.factor (edlevel $)+$ married + kids,

summary (fit_cop_POI)

data $=$ rwm5yr, marginal $=$ poisson $. \operatorname{marg}, \mathrm{id}=\mathrm{id}$,

cormat $=$ cluster. $\operatorname{cormat}($ id, type $="$ exchangeable" $))$

t $2=$ Sys.time ()

\#MODELO DE COPULAS GAUSSIANAS BINOMIAL NEGATIVO

t3 $=$ Sys.time ()

fit_cop_NB $=$ gcmr $($ docvis $\sim$ hospvis+age+outwork + female+as.factor $($ edlevel $)+$ married + kids,

data $=$ rwm5yr, marginal $=$ negbin.marg, $\mathrm{id}=\mathrm{id}$,

summary (fit_cop_NB)

$$
\text { cormat }=\text { cluster } . \text { cormat }(\text { id, type="exchangeable" }) \text { ) }
$$

$\mathrm{t} 4=$ Sys.time ()

t $5=$ Sys.time ()

fit_cop_NB $=$ gcmr $($ docvis $\sim$ hospvis + age + outwork + female + as.factor $($ edlevel $)+$ married + kids

summary (fit_cop_NB)

data $=$ rwm5yr, marginal $=$ negbin $. \operatorname{marg}, i d=i d$,

cormat $=$ cluster. cormat $($ id, type="independence" $))$

t6 $=$ Sys.time ()

t $7=$ Sys.time ()

fit_cop_NB =gcmr (docvis $\sim$ hospvis+age+outwork+female+as.factor (edlevel) + married + kids, data $=$ rwm5yr, marginal = negbin.marg, $\mathrm{id}=\mathrm{id}$,

summary (fit_cop_NB) cormat $=$ cluster. $\operatorname{cormat}($ id, type $="$ ar $1 "))$

t $8=$ Sys.time ()

t9 $=$ Sys.time ()

fit_cop_NB $=$ gcmr $($ docvis $\sim$ hospvis + age + outwork + female + as.factor $($ edlevel $)+$ married + kids, data $=$ rwm5yr, marginal $=$ negbin.marg, $\mathrm{id}=\mathrm{id}$, cormat $=$ cluster. cormat $($ id , type $=$ "unstructured" $)$ )

summary ( fit cop NB)

t10 $=$ Sys.time $(\overline{)}$

\section{B.3.2 SAS}

PROC GENMOD DATA=WORK.RWM5YR

$\operatorname{PLOTS}(\mathrm{ONLY})=\mathrm{ALL}$;

CLASS ID YEAR;

MODEL DOCVIS = HOSPVIS AGE OUTWORK FEMALE EDLEVEL2 EDLEVEL3 EDLEVEL4 MARRIED

KIDS / LINK=LOG DIst=poiss on TYPE3;

REPEATED SUBJECT=ID / SORTED WITHINSUBJECT=year TYPE-EXCH COVB CORRW MODELSE; RUN;

PROC GENMOD DATA-WORK.RWM5YR

$\operatorname{PLOTS}(\mathrm{ONLY})=\mathrm{ALL}$

CLASS ID year;

MODEL DOCVIS = HOSPVIS AGE OUTWORK FEMALE EDLEVEL2 EDLEVEL3 EDLEVEL4 MARRIED

KIDS / LINK=LOG DISt=negbin TYPE3;

REPEATED SUBJECT=ID / SORTED WTTHINSUBJECT=year TYPE-EXCH COVB CORRW MODELSE; RUN;

PROC GENMOD DATA-WORK.RWM5YR

$\operatorname{PLOTS}(\mathrm{ONLY})=\mathrm{ALL}$;

CLASS ID year;

MODEL DOCVIS $=$ HOSPVIS AGE OUTWORK FEMALE EDLEVEL2 EDLEVEL3 EDLEVEL4 MARRIED KIDS / LINK=LOG DISt=negbin TYPE3; 
REPEATED SUBJECT=ID / SORTED WITHINSUBJECT=y ear TYPE=AR COVB CORRW MODELSE; RUN;

PROC GENMOD DATA-WORK.RWMSYR

$\operatorname{PLOTS}(\mathrm{ONLY})=\mathrm{ALL}$

CLASS ID year;

MODEL DOCVIS = HOSPVIS AGE OUTWORK FEMALE EDLEVEL2 EDLEVEL3 EDLEVEL4 MARRIED KIDS / LINK=LOG DISt=negbin TYPE3;

REPEATED SUBJECT=ID / SORTED WITHINSUBJECT=year TYPE=IND COVB CORRW MODELSE; RUN;

PROC GENMOD DATA-WORK.RWMSYR

$\operatorname{PLOTS}(\mathrm{ONLY})=\mathrm{ALL}$;

CLASS ID year;

MODEL DOCVIS = HOSPVIS AGE OUTWORK FEMALE EDLEVEL2 EDLEVEL3 EDLEVEL4 MARRIED

KIDS / LINK=LOG DISt=negbin TYPE3;

REPEATED SUBJECT=ID / SORTED WITHINSUBJECT=y ear TYPE-UNSTR COVB CORRW MODELSE; RUN;

PROC GENMOD DATA-WORK.RWM5YR

$\operatorname{PLOTS}(\mathrm{ONLY})=\mathrm{ALL}$

CLASS ID year;

MODEL DOCVIS $=$ HOSPVIS AGE OUTWORK FEMALE EDLEVEL2 EDLEVEL3 EDLEVEL4 KIDS LINK=LOG DISt=negbin TYPE3;

REPEATED SUBJECT $=$ ID / SORTED WITHINSUBJECT $=$ year TYPE $=$ IND COVB CORRW MODELSE; RUN;

PROC GENMOD DATA-WORK.RWMSYR

$\operatorname{PLOTS}(\mathrm{ONLY})=\mathrm{ALL}$;

CLASS ID year;

MODEL DOCVIS = HOSPVIS AGE OUTWORK FEMALE EDLEVEL3 EDLEVEL4 KIDS / LINK=LOG

DISt $=$ negbin TYPE3;

REPEATED SUBJECT=ID / SORTED WITHINSUBJECT=year TYPE=IND COVB CORRW MODELSE; RUN; 
APÊNDICE B 


\section{Referências Bibliográficas}

Akaike (1973) Htrotugu Akaike. Maximum likelihood identification of gaussian autoregressive moving average models. Biometrika, 60:255-265. Citado na pág. 19

Artes (1997) Rinaldo Artes. Extensões da Teoria das Equaçoes de Estimação Generalizadas a Dados Circulares e Modelos de Dispersão. Tese de Doutorado, Instituto de Matemática e Estatística da Universidade de São Paulo, 25/04/97. Citado na pág. 7

Barndorff-Nielsen e Jørgensen (1991) Ole E Barndorff-Nielsen e Bent Jørgensen. Some parametric models on the simplex. Journal of Multivariate Analysis, 39:106-116. Citado na pág. 7

Breslow (1996) Norman E Breslow. Generalized linear models: checking assumptions and strengthening conclusions. Statistica Applicata, 8:23-41. Citado na pág. 49

Cameron e Trivedi (1986) A Colin Cameron e Pravin K Trivedi. Econometric models based on count data. comparisons and applications of some estimators and tests. Journal of Applied Econometrics, 1:29-53. Citado na pág. 21

Chang (2000) Yue-Cune Chang. Residuals analysis of the generalized linear models for longitudinal data. Statistics in Medicine, 19:1277-1293. Citado na pág. 17

Cook (1977) R Dennis Cook. Detection of influential observation in linear regression. Technometrics, 19:15-18. Citado na pág. 18

Crowder (1987) Martin Crowder. On linear and quadratic estimating functions. Biometrika, 74: 591-597. Citado na pág. 8, 9

Cui et al. (2007) James Cui et al. QIC program and model selection in GEE analyses. Stata Journal, 7:209. Citado na pág. 30

Cui e Feng (2008) Jisheng Cui e Liyun Feng. Correlation structure and model selection for negative binomial distribution in gee. Communications in Statistics-Simulation and Computation, 38:190-197. Citado na pág. 2, 21, 30, 38, 44

Dunn e Smyth (1996) Peter K Dunn e Gordon K Smyth. Randomized quantile residuals. Journal of Computational and Graphical Statistics, 5:236-244. Citado na pág. 27

Fotouhi (2008) Ali Reza Fotouhi. Modelling overdispersion in longitudinal count data in clinical trials with application to epileptic data. Contemporary Clinical Trials, 29:547-554. Citado na pág. 50

Godambe (1960) Vidyadhar P Godambe. An optimum property of regular maximum likelihood estimation. The Annals of Mathematical Statistics, 31:1208-1211. Citado na pág. 8

Godambe (1991) Vidyadhar P Godambe. Estimating functions. Oxford University Press, Oxford, UK. Citado na pág. 8 
Greenwood e Yule (1920) Major Greenwood e G Udny Yule. An inquiry into the nature of frequency distributions representative of multiple happenings with particular reference to the occurrence of multiple attacks of disease or of repeated accidents. Journal of the Royal statistical society, 83:255-279. Citado na pág. 21

Hardin e Hilbe (2003) James W Hardin e Joseph M Hilbe. Generalized Estimating Equations. Wiley Online Library. Citado na pág. 30

Hilbe (2014) Joseph M Hilbe. Modeling Count Data. Cambridge University Press. Citado na pág. 4, 29,68

Hilbe (2011) Joseph M Hilbe. Negative Binomial Regression. Cambridge University Press. Citado na pág. $1,21,22,30,68,74$

Johnston e Stokes (1996) Gordon Johnston e M Stokes. Repeated measures analysis with discrete data using the sas system. SAS Institute Inc., Cary, NC. Citado na pág. 13

Jørgensen e Labouriau () Bent Jørgensen e Rodrigo Labouriau. Exponential families and theoretical inference. Lecture Notes. Citado na pág. 7

Lawless (1987) Jerald F Lawless. Negative binomial and mixed poisson regression. Canadian Journal of Statistics, 15:209-225. Citado na pág. 25

Liang e Zeger (1986) Kunkg-Yee Liang e Scott L. Zeger. Longitudinal data analysis using generalized linear models. Biometrika, 73:13-22. Citado na pág. 1, 7, 12, 14, 50

Lipsitz et al. (1991) Stuart R Lipsitz, Nan M Laird e David P Harrington. Generalized estimating equations for correlated binary data: using the odds ratio as a measure of association. Biometrika, 78:153-160. Citado na pág. 7

Masarotto et al. (2012) Guido Masarotto, Cristiano Varin et al. Gaussian copula marginal regression. Electronic Journal of Statistics, 6:1517-1549. Citado na pág. 30, 31, 32

Pan (2001) Wei Pan. Akaike's information criterion in generalized estimating equations. Biometrics, 57:120-125. Citado na pág. 17, 19, 30

Paula (2013) Gilberto Alvarenga Paula. Modelos de Regressão: Com Apoio Computacional. IMEUSP São Paulo. Citado na pág. 17, 22, 25, 26

Preisser e Qaqish (1996) John S Preisser e Bahjat F Qaqish. Deletion diagnostics for generalised estimating equations. Biometrika, 83:551-562. Citado na pág. 17

Prentice (1988) Ross L Prentice. Correlated binary regression with covariates specific to each binary observation. Biometrics, 44:1033-1048. Citado na pág. 13

Snedecor e Cochran (1967) George W Snedecor e WG Cochran. Statistical Methods. Iowa State University Press, 327:12. Citado na pág. 2

Song et al. (2004) Peter X-K Song, Zhenguo Qiu e Ming Tan. Modelling heterogeneous dispersion in marginal models for longitudinal proportional data. Biometrical Journal, 46:540-553. Citado na pág. 7

Song (2000) Peter Xue-Kun Song. Multivariate dispersion models generated from gaussian copula. Scandinavian Journal of Statistics, 27:305-320. Citado na pág. 31, 32

Song e Tan (2000) Peter Xue-Kun Song e Ming Tan. Marginal models for longitudinal continuous proportional data. Biometrics, 56:496-502. Citado na pág. 7 
Thall e Vail (1990) Peter F Thall e Stephen C Vail. Some covariance models for longitudinal count data with overdispersion. Biometrics, 46:657-671. Citado na pág. 50

Trindade (2014) Daniele de Brito Trindade. Modelagem para Dados Longitudinais de Contagem. Tese de Doutorado, Universidade Federal de Pernambuco. Citado na pág. 30, 32

Venezuela (2003) Maria Kelly Venezuela. Modelos lineares generalizados para análise de dados com medidas repetidas. Tese de Doutorado, Instituto de Matemática e Estatística da Universidade de São Paulo. Citado na pág. 7, 17, 18

Venezuela (2007) Maria Kelly Venezuela. Equação de estimação generalizada e influência local para modelos de regressão beta com medidas repetidas. Tese de Doutorado, Universidade de São Paulo. Citado na pág. 12, 19

Venezuela et al. (2007) Maria Kelly Venezuela, Denise Aparecida Botter e Mônica Carneiro Sandoval. Diagnostic techniques in generalized estimating equations. Journal of Statistical Computation and Simulation, 77:879-888. Citado na pág. 7, 17

Venezuela et al. (2011) Maria Kelly Venezuela, Mônica Carneiro Sandoval e Denise Aparecida Botter. Local influence in estimating equations. Computational Statistics 85 Data Analysis, 55: 1867-1883. Citado na pág. 17

Weiss (2005) Robert E. Weiss. Modeling Longitudinal Data: With 72 Figures. Springer Science \& Business Media. Citado na pág. 1

Zeger e Liang (1992) Scott L Zeger e Kung-Yee Liang. An overview of methods for the analysis of longitudinal data. Statistics in Medicine, 11:1825-1839. Citado na pág. 49

Zorn (2001) Christopher JW Zorn. Generalized estimating equation models for correlated data: A review with applications. American Journal of Political Science, 45:470-490. Citado na pág. 7 